DEVELOPING POST-ABLEIST MUSIC THERAPY:

\title{
AN AUTOETHNOGRAPHY EXPLORING THE COUNTERPOINT OF A THERAPIST EXPERIENCING ILLNESS/DISABILITY
}

BY

\section{CAROLYN MAY SHAW}

A Thesis Presented in Fulfilment of the Requirement for the Degree of Doctor of Philosophy in Music

New Zealand School of Music, Te Kōkī, Victoria University of Wellington, New Zealand 


\section{Abstract}

This $\mathrm{PhD}$ examined a therapist's experience of illness/disability to see if any new light could be shed on music therapy whilst also finding ways to navigate disability as a practitioner. There has not been adequate research attention given to the experiences of music therapists who have an illness/disability. The position is often negotiated in isolation with minimal tools and resources. An arts-based autoethnography was used to determine how the close examination of one's personal experience with illness/disability can impact on practice, how the work can be negotiated, and to uncover any new practical or theoretical meanings. Furthermore, it looked to determine what arts-based autoethnography could offer one's practice. A poststructural lens was used that drew on social constructionism, feminism, and the work of Michel Foucault. Data generated from a music therapist's practice, experiences of illness/disability, literature, and professional documents were analysed using Foucault's “critical ontology of ourselves” (Foucault, 1984b, p. 47).

Hidden processes of problematic ableism were found within the practice examined as well as in some educational and professional encounters. These regimes of ableism were supported by universalising and dichotomising discourses, namely humanism, western normativity, limited observable understandings of disability, and the enforcement of able/disabled divide through many binaries. The methodology provided the tools to reposition the practice to politicise disability and address ableism.

Addressing ableism was found to be more complex than simply incorporating disability issues into existing contemporary frameworks. The analysis led to the development of Post-Ableist Music Therapy (PAMT). PAMT extended the relational ethic beyond what was present in the prior practice by drawing on aspects of posthumanism, agonistic plurality, and increasing the visibility of disability studies and crip theory. Therefore, PAMT offers a different lens to the critical orientations' apparatus: a social justice practice not based on empowerment and humanism but on agonism and posthumanism instead. As there is a lag in the theorisation of ableism, PAMT provides an alternative framework that can be applied to current approaches to increase our professional consciousness of ableism.

By repositioning the practice and exploring alternative subjectivities, the professional and personal narratives of a therapist experiencing illness/disability became more 
integrated, working with-not against-each other in a shared activism. The methodology fostered an increased ethical care of the self; offered tools that critiqued what we are; experimented with going beyond the limits imposed on us. The use of such tools could have wider application in the everyday practices of therapists. The findings have significant implications for practice and training, as the challenges people and societies face cannot be adequately dealt with without tools to explicitly uncover and address normalisation and ableism. 


\section{Acknowledgements}

Although this research bears my name, it is not the product of one person.

Regretfully, I do not have enough words to name everyone. This research would not have been the same without those who have guided me, talked with me, and laughed with me. Nor would it have been the same without the pauses during bodily upheavals and becoming a parent. This research has meant more to me than the content between the cover pages. What I have learned from and with you all has changed me in many ways. I am forever grateful.

- Associate Professor Sarah Hoskyns and Associate Professor Sue Cornforth (PhD supervisors): your ongoing belief in me planted seeds of possibility. I appreciated your kind, flexible approach and insights. You have taught me to think in many different ways.

- The McKenzie Music Therapy Scholarship and the Marion Rayward Memorial Scholarship in Music: Without this generous funding, this research would not have been possible.

- Laura Kamau (Māori learning advisor): your ability and willingness to share Māori understandings of health offered an important perspective.

- Claire Molyneux (clinical supervisor): the space you provided allowed me to grapple with disability related issues in practice.

- Dr Kimberly Cannady (ethnomusicology lecturer): it was a joy to discover the links with and new insights from ethnomusicology.

- Bevan (husband): for everything!

- Frida (daughter): for the breaks to jump in muddy puddles.

- Esmay (daughter): for the cuddles.

- My wider Whānau: for making me dinners, always being there, and keeping things fun.

- Anna Moore and Kimberley Wade: for never letting me shy away from what was unpalatable or unspeakable.

- Schools, facilities, families, and the people I have worked with: none of this would have been possible without meeting you.

In memory of Mrs. McLeod, a music teacher who shaped the lives of many during their formative school years. 


\section{Statement of ethical approval}

The research was considered and approved by the Standing Committee of the Human

Ethics Committee, Victoria University. Approval \#: 22214, November 2015 


\section{Table of contents}

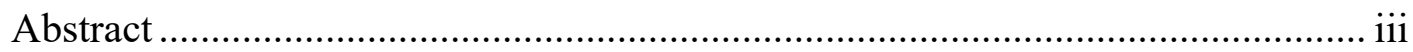

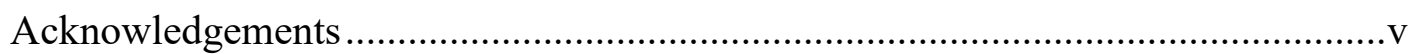

Statement of ethical approval......................................................................... vi

Table of contents ........................................................................................... vii

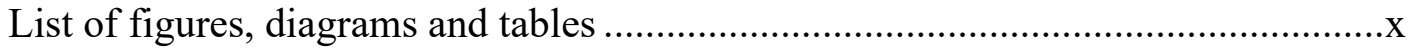

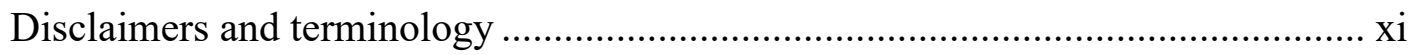

Chapter 1: Introduction........................................................1

1. Background: A personal and professional jolt ..................................................2

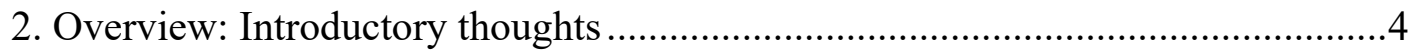

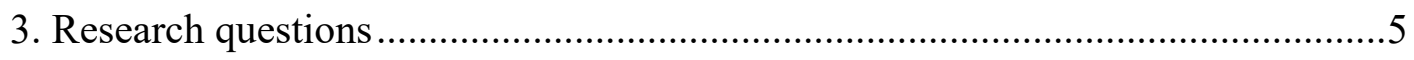

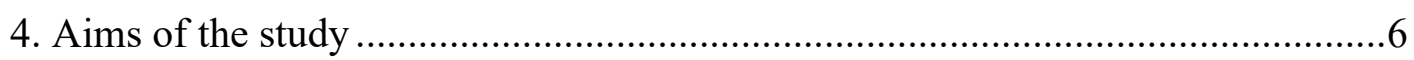

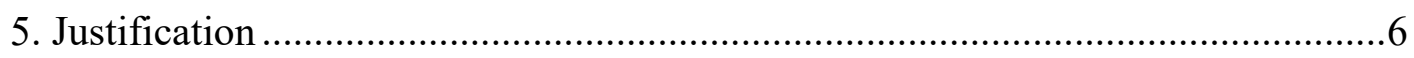

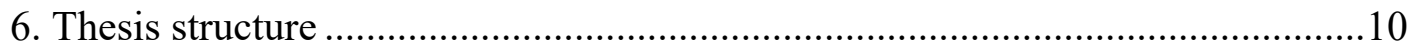

Chapter 2: Literature Review: A Patient History ................................11

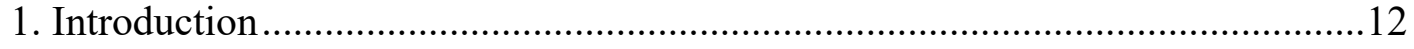

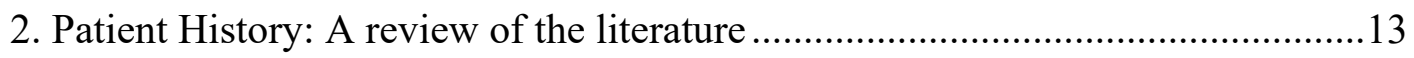

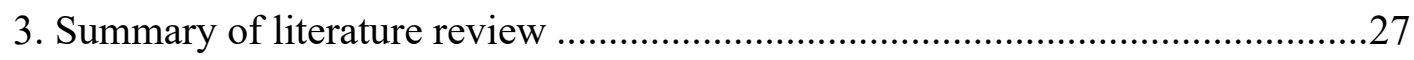

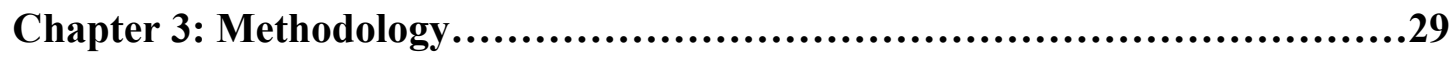

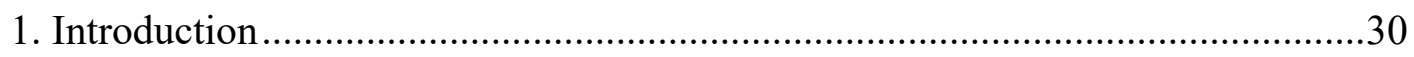

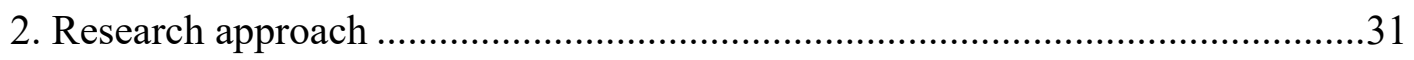

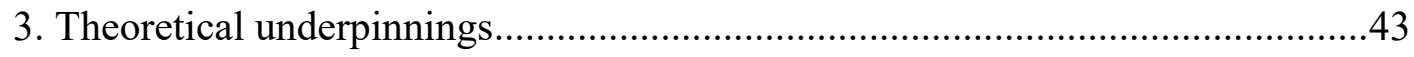

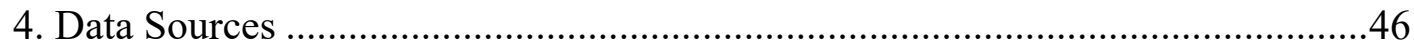

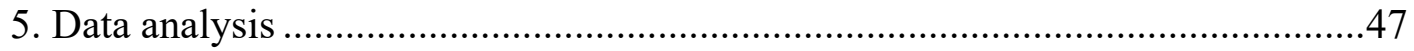

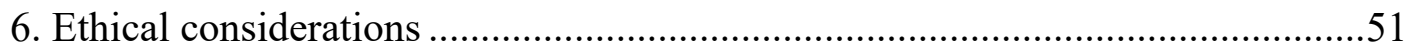

7. Prelude to, and structure of, the following analysis chapters............................53

Chapter 4: Seeing the Unseen: The Shadow Puppets of Disability and Illness...55

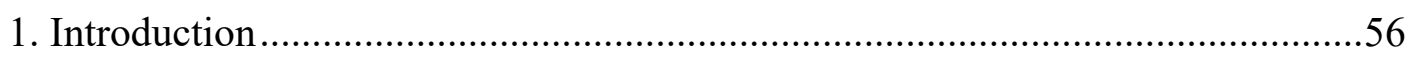

2. "Truth": Disability and illness are easily identifiable ......................................61

Chapter 5: Disclosure: To Hide or Not to Hide in the Box?..................................80

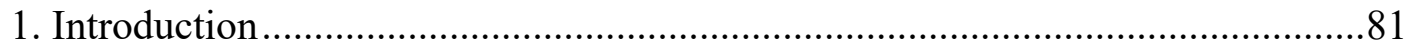

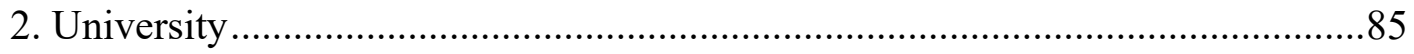

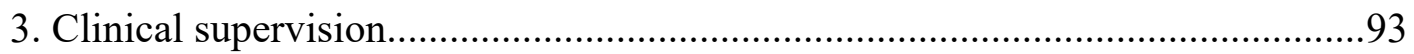


Chapter 6: Falling in the Space Between: Oppositional Binaries

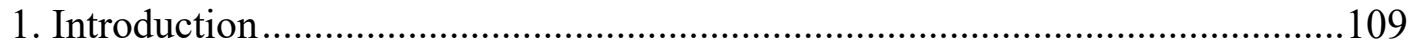

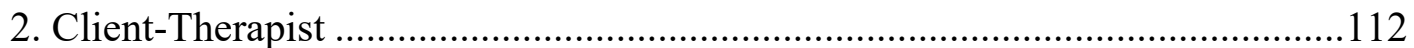

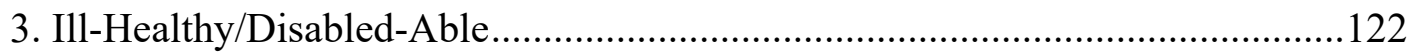

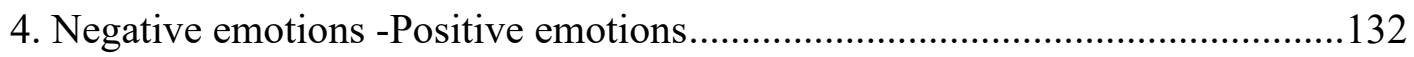

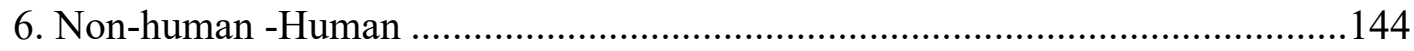

Chapter 7: Finding Ladders: Success.......................................156

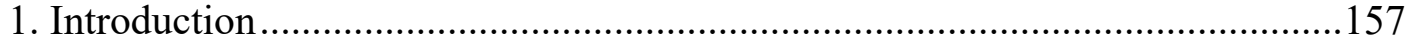

2. "Truth" 1: The ability to meet normalised standards determines the level of success

3. "Truth" 2: Success can be measured ................................................................ 170

4. "Truth" 3: Success is predictable and therefore can be planned........................182

\section{Chapter 8: Creating the Self as a Work of Art (Part One):}

Developing Post-Ableist Music Therapy

1. Introduction: Searching for a way to work without a vegetarian having

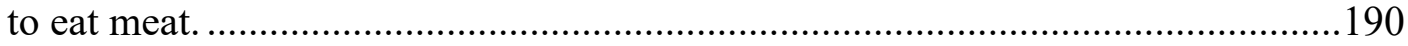

2. Starting with the person: From human to posthuman .......................................195

3. Person meets music: The musical posthuman....................................................196

4. Putting it all together: Post-Ableist Music Therapy (PAMT) ...........................205

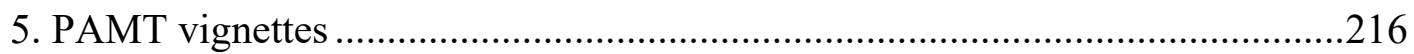

\section{Chapter 9: Creating the Self as a Work of Art (Part Two):}

Changing Subjectivity Through Illness/Disability, Music and Research........226

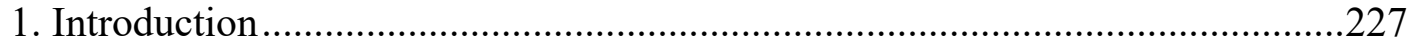

2. Constructing a new home: Changing subjectivity through illness/disability, music and research to subvert internalised ableism.........................................229

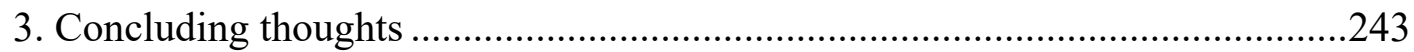

Chapter 10: Discussion......................................................244

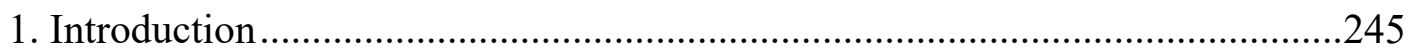

2. What different light does a poststructural analysis of the narratives shed

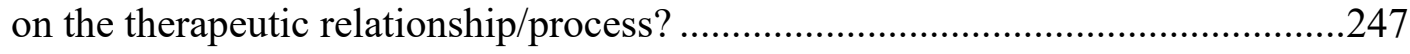

3. How did the close examination of a music therapist's experience of illness/disability impact on their professional practice? 
4. How can the work of therapy be managed when client and practitioner-patient share similar illness/disability narratives?

5. What does the method/writing of a poststructurally-informed art-based autoethnography offer one's practice?

\section{Chapter 11: Final thoughts: Reflections, Recommendations and}

Conclusion.

1. Reflections on the research process: Challenges and limitations

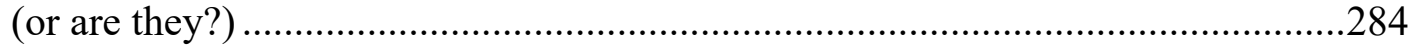

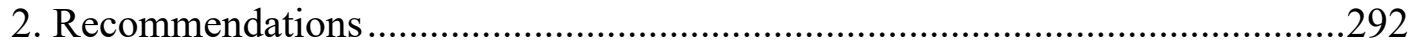

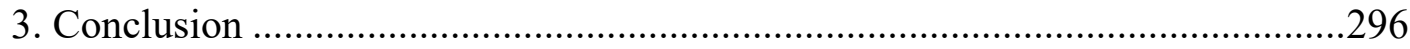

References....................................................................300

Appendices.................................................................345

Appendix 1: Examples of information and consent forms ..................................345

Appendix 2: Co-written music therapy report example.......................................356

Appendix 3: Notes and recommendations to the Standards of

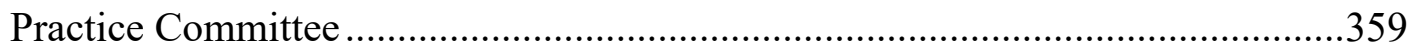




\section{List of figures, diagrams and tables}

FIGURE 1: ETHICAL CONCERNS DERIVED FROM DOMINANT DISCOURSES IN THE PRACTICE

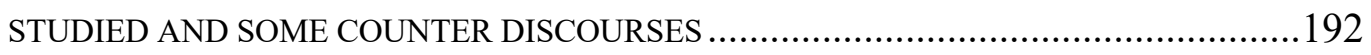

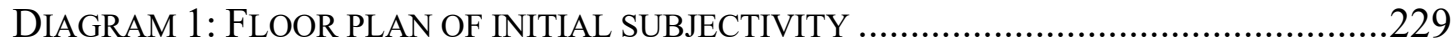

DIAGRAM 2: RENOVATIONS OF SUBJECTIVITY FLOOR PLAN _...................................230

TABLE 1: FONTS USED FOR PERSONAL EXPERIENCES DURING ANALYSIS CHAPTERS ......54

TABLE 2: EXAMPLES OF POTENTIAL KEY COMPETENCY OBJECTIVES FOR MARCUS WITHIN HUMANISM AND POSTHUMANISM ......................................................22

TABLE 3: COMPARISON OF THE NUMBER OF HITS FOUND FOR TERMS RELATING TO OPPRESSION WITH "MUSIC THERAPY" ON THE 27/08/2018. 250 


\section{Disclaimers and terminology}

1. Though I am able to, I have not musically notated audio examples: I do not wish to create a barrier to matters I intend to illuminate for a broad audience, including those with disabilities for whom music education has been inaccessible.

2. For accessibility to blind and low vision readers, the format does not adhere to APA guidelines. I have been guided by the recommendations by the New Zealand Blind Foundation. However, I acknowledge that there are some sections that have not met all accessibility criteria due to the way the content needed to be presented. I have, in these moments, given descriptions of proceeding structural changes to alert readers in the hope that it will be easier to follow.

3. I deliberately change between using the person-first language of "person with a disability", and the political reclaiming of disability as "disabled person/people". Person-first language stresses the importance of the person rather than the category, whereas identity-first language wishes to acknowledge that one cannot separate themselves from their disability (it is not a shameful aspect that negates them from being a person). As I explore multiple positions throughout this thesis, and advocate for plural identities, I use both accordingly.

4. I refer to chronic illness as an impairment and disability in this thesis. Therefore, the terms "disabled" and "illness" are used interchangeably. When disability is considered to be dynamically produced-by that which is physical/biological and that which is social, cultural and environmental-it makes little sense to separate illness from disability. People with chronic illnesses are exposed to forms of social exclusion, disadvantage and disablism, like those with other impairments, whether or not they identify as disabled (Thomas, 2007). The terms "impairment" and "disability" are also used interchangeably due to the poststructural critique of impairment not being the sole domain of the body/mind and the inseparableness of impairment and disability.

5. I struggled with the terminology for practitioners who experience illness/disability. The term practitioner-patient was predominantly used in my research proposal and earlier chapters of this thesis. The term includes both 
physical and mental health. However, "patient" and "client" have been challenged by the E-Patient movement and music therapy (McFerran, 2012) and my awareness and identification with disabled identities increased. As practitioner-patient became less appropriate, I stopped using this term in later chapters of the thesis. I have not amended this, nor sought to use one consistent term. I have instead left this inconsistency to give a sense of the different and changing positions throughout. 


\section{Chapter 1}

\section{Introduction}

"Leaving for work": painting by my husband Bevan ${ }^{1}$

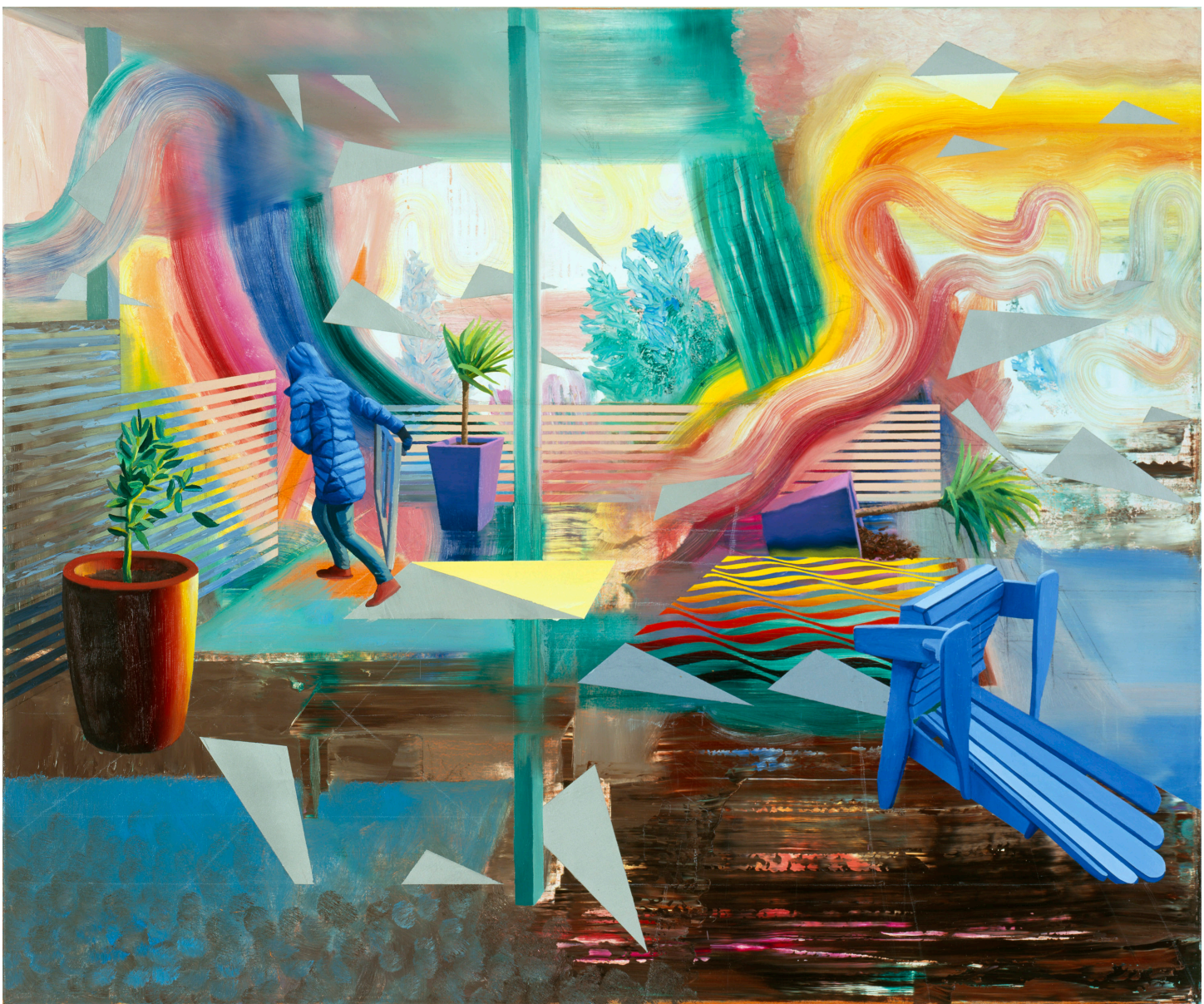

[Colourful painting of a person leaving in a hurry through a gate. On one side a chair and plant have fallen over from a rainbow wind gush.]

\footnotetext{
${ }^{1}$ Each chapter starts with a photograph/image from my life that encapsulates some ideas in the chapter.
} 


\section{Background: A personal and professional jolt}

\section{1st journal entry 2011}

In my student days, music therapy "training-wheels" kept me moving, creating motion despite not always knowing what I was doing, or where the intertwining of melodies would take us. I fought against the constant selfdoubt that my intentions of "helping" would not flower, that I would be left with a seed I could not grow.

As time went by, I watched as flowers bloomed bright reds and soft yellows. Their flowering once a surprise began to take on a more predictable manner. With this came the expectation that through the process of creative sound we would grow in the warm sun. Doubt sunk with the rise of deep-seated trust that intended outcomes transpire to some extent, no matter how small, in such conditions. But you don't know what you don't know. What I thought about my work and the stable foundations I had built were challenged when diagnoses were added like a list of converse credentials.

Prior to foundations rocking I danced to the sound of Mozart concertos, rich in order and predictability. It was a time when dissonance would always resolve as the energy to reach the tonic would prevail. I played Bach's Brandenburg concertos, smiling with the comfort of knowing the familiar.

But some things change, with illness an abrupt shift to the atonal rendered me scratching my head with how to dance. The music of life that now surrounded me was unfamiliar, unpredictable, chaotic with no lovely resolutions to the stable tonic: direction thrown off course by the wind of dissonance. I played loud note clusters on the piano and wanted to burn it like Diego Stocco.

My world had changed and with it came new glasses that sharpened the previously blurry smudges. I wanted to take them off, but they were super glue cemented. My client's flowers were no longer "whole". I saw the missing petals, the holes left from insects' lunches. Their bright colours 


hiding the subtler things that were not seen before: their struggle within the
garden for a place, for sun, for water. What was important had changed. It
was not about getting the seed to flower. It may not flower like the others,
but form a strong stem for life around it with a beauty no less. It may not
flower but become something else: not a broken flower.
Some things change: I am unwell. Some stay the same: I am a music
therapist. When one element changes the ripple effect is inevitable. I am
back to that place of "training-wheels", I am learning it all again.
With pharmaceuticals rattling, I have re-trained my body to sit up for more
than two minutes, to stand and to walk some. Now I am learning to do my
job with the new glasses.
When I first listened to atonal music it annoyed me and made no sense. But
with time came an appreciation of what 12-tone music had to offer: its joy
and beauty growing from a place of initial dislike. So, I pick up my pencil
and scribble with the hope that each mark will create understanding and
with this the discomfort of my ambiguous thoughts will ease.

This research journey started with the journal entry above: after returning to work from a period of sick leave in 2010. I wondered why my practice suddenly felt so different, and what to do about it. Like the feeling in the painting above, going to work felt like a constant struggle. Developing a chronic illness may challenge one's previous world-view and identity (Charmas, 1999; Karnilowicz, 2001), cause "cognitive dissonance" (Birk, 2013), expose aspects of oppression caused by disability, and reveal the previous "unearned privileges"2 of being able-bodied (Birk, 2013; McIntosh, 1988). Consequently, I wrote many more journal entries after this one. Writing can enhance the reflexive process of sense-making, which is intensified when an individual experiences dissonance in the normal flow of daily life (Boylorn

\footnotetext{
2 The concept of "unearned privileges" was developed from Du Bois' idea of "psychological wage" for white laborers that gave them special status to poor blacks (Du Bois, 1972). The concept was developed further by Peggy McIntosh in her 1988 working paper titled, "White privilege and male privilege: A personal account of coming to see correspondences through work in women's studies".
} 
\& Orbe, 2014a), and produce "new practices that serve the writer's life purposes and challenge the communities in which she lives" (Kamler, 2001, p. 54). With the help of my MT supervisor, I realised that I was developing a large area of inquiry focusing on the different lens that illness/disability brought to my work and the role of creativity (such as writing) in developing understanding and ways of living. Indeed, the interest that there might be more to be uncovered in the meaning of illness, disability and consequently music therapy (MT) intrigued me. I commenced this study two and a half years after this initial entry.

To provide background to the shaping of my early practice and the differentness I felt post 2010, I trained at a time (between 2004-2006) when critical approaches were beginning to gain traction within the profession, but had yet to yield significant engagement in New Zealand (NZ). Medical understandings of disability were prevalent in assigned readings and humanism framed a lot of course content. I then practiced as a music therapist from 2007 under the NZ MT Code of Ethics and practice standards that were framed by the medical model of disability. My work predominantly centered on working with children and their families within school and home contexts. Prior to illness, my identity was that of an able-bodied, Pākehā, cisgendered, heterosexual woman from a middle-class family.

\section{Overview: Introductory thoughts}

The position of practitioners who experience illness/disability offers a differing perspective to examine understandings of therapeutic practice. Despite disability and illness being the focus of much attention in MT, illness/disability within the profession is often not acknowledged or discussed, and lacks research attention. My research explores how the close examination of one's illness/disability can have an impact on the understanding and implementation of practice and the experience of being a practitioner-patient (PP).

Where the patient-and-therapist-self meet may pose a challenge for the person, and/or present an opportunity for professional and personal development (Goldberg, HadasLidor, \& Karnieli-Miller, 2014). One's MT work is influenced by life experiences, and vice-versa (Hadley, 2001, 2003), and both personal and professional experiences significantly influence professional growth (Rønnestad \& Skovholt, 2003). Therefore, 
a process supporting personal-professional integration (Rønnestad \& Skovholt, 2003), and the understanding of one's intersectional and shifting identities, may be beneficial. The current study sought to integrate personal experience and professional knowledge through the creative process: an arts-based autoethnography with a Foucauldian analysis.

Autoethnographic and arts-based approaches to research are increasing in MT research. However, they have previously had scant attention in the discipline comparative to other areas of social science (D. Bakan, 2014; Ledger \& Edwards, 2011) and are still in their infancy (Viega \& Forinash, 2016). Additionally, within the confines of this study, no MT arts-based autoethnographies were found that utilised a Foucauldian form of analysis. Therefore, I examined what new knowledge emerges if this different theoretical approach is taken, as well as how autoethnography could support my practice and research, thereby showing the potential of the methodology to be a supportive tool on accounts of both research and practice.

The primary aim is to cultivate new meanings, understandings, and therapeutic practices to support good services for clients. I seek to extend the limits of what is thinkable, in order to enhance the contestability of what we take to be natural and 'given' about our current ways of relating to ourselves and others in the therapy space (Rose, 1996). Although this research refers to MT, it is intended to be useful for and contribute to multiple disciplinary and academic fields. The implications could be of interest to allied health professionals, doctors, patients, educators, theoreticians, music therapists, and other areas, such as disability studies.

\section{Research questions}

1. How does the close examination of a music therapist's experience of illness/disability impact on their professional practice?

2. How can the work of therapy be managed when client and practitioner-patient share similar illness/disability narratives?

3. What different light does a poststructural analysis of these narratives shed on the therapeutic relationship/process?

4. What does the method/writing of a poststructurally-informed art-based autoethnography offer one's practice? 


\section{Aims of the study}

1. To open up and cultivate new meanings and new practices

2. To illuminate how societal ideas about illness and disability may have an impact on the therapeutic process

3. To foster 'care of the self' within practice

4. To support ways of integrating professional knowledge and personal experience

5. To decrease the near silence around PPs in MT and other related professions

\section{Justification}

I have identified four main reasons as to why this research is necessary:

- The paucity of literature regarding therapists with disabilities in MT

- To hear previously silenced voices: To diversify practice

- To contribute to our understanding of how therapists' life events shape practice and impact on professional identity

- To provide further tools that may support reflexivity

In summary, there is a scarcity of literature, research, and active voices heard in MT relating to therapists who experience illness/disability. This contributes to a lack of resources and tools, professional displacement, and marginalisation. Cultivating further space in the profession for PPs' experiences and perspectives to be voiced may support the current call in the profession to diversify practice. Exploring different and emerging tools and theoretical approaches may lead to the development of new practices. A more detailed explanation of the four justifications follows.

\subsection{Paucity of literature regarding therapists with disabilities/illness}

There is a paucity of literature regarding PPs within MT. In the context of NZ, apart from one article (Webster \& Rickson, 2018), there is no research regarding the experiences of PPs ${ }^{3}$. However, anecdotally, there seems to be interest for this research

\footnotetext{
${ }^{3}$ The experience of a therapist having a family member with a disability is, however, discussed in the 'New Zealand Journal of Music Therapy' (Rickson, 2004).
} 
area: colleagues have discussed with me the impact of their own, or their loved one's, experiences of illness/disability after inquiring about my research.

There is a small amount of international literature. It indicates that key figures in MT, such as Clive Robbins (Hadley, 2003) and Mary Priestley (Hadley, 2001), and other engaging writers (Dunn, 2009; LaCom \& Reed, 2014) have, in part, had their understandings of practice shaped by their experiences of illness/disability. It is interesting that therapists' experiences of illness/disability may in fact be a contributing force in theoretical knowledge, yet it receives such little research attention. Hadley (2017) gives anecdotal examples of microaggressions experienced by therapists who have a disability, but a large-scale inquiry into such professional discrimination is lacking. Most literature that refers to the position of PP often mentions it briefly and is not the main focus research.

This inadequate attention signifies a lack of literary support and contributes to marginalisation. The underrepresentation of the experiences of PPs in the literature may hinder feelings of professional inclusion and acceptance. It has been acknowledged that some PPs want to know that they are not the only person managing this position (Velde, 1999). Feelings of isolation may occur when there is silence, or networks are not established within a profession (Velde, 1999). For the most part, the literature from multiple disciplines suggests that PPs are managing the position in isolation and relative secrecy, which can result in self-stigma and shame (Zerubavel \& O’Dougherty-Wright, 2012). Increasing the visibility of practitioner disability through literature and research may help transgress marginality (Chacala, McCormack, Collins, \& Beagan, 2013) and isolation.

\subsection{Hearing previously silenced voices: diversifying practice}

Relatedly, in order to enhance the contestability of normalised therapeutic practices and open up new understandings, there is a need to hear voices that are often silenced. It can be difficult for therapists who are not in the dominant group to counter takenfor-granted ideas in our profession (Hadley, 2013a). Silence occurs because they do not feel comfortable or safe in sharing their experiences as they speak from a less privileged position (Hadley, 2013a). Despite barriers to voicing experiences, PPs may bring unique and valuable perspectives on disability and illness (Chacala et al., 2013) 
that can assist in diversifying practice. Their perspective may invite reflection on how disability is understood and approached within a profession (Chacala et al., 2013).

There is an active call to increase diversity and respectful openness to difference within creative arts therapies (Hadley, 2013a, 2014) and other allied health professions (Chacala et al., 2013). Within MT there has been movement away from the medical model that grounded much practice in the $20^{\text {th }}$ century towards practices that foster social change, value diversity, and/or approach disability from other perspectives (Rickson, 2014d). An increased platform has emerged since my training for diverse voices and engagement with critical lenses. There are many critical and social justice approaches, such as feminist MT (Edwards, 2006; Edwards \& Hadley, 2007), activist approaches (Curtis, 2012), creative and anti-oppressive practices (Baines, 2013; J. Smith, 2012), queer MT (Bain, Grzanka, \& Crowe, 2016; Boggan, Grzanka, \& Bain, 2017; Whitehead-Pleaux et al., 2012), resource orientated MT (Rolvsjord, 2010), and community MT (Pavlicevic \& Ansdell, 2004; Vaillancourt, 2012). Recent and proposed conferences in the profession are embracing the theme of diversifying practice, such as the Australian MT Association national conference 2018, and the proposed theme for the world congress of MT conference (2020), "The polyrhythms of music therapy". Some journals actively seek to represent a full range of positions and diverse practices. Given this current interest, and the slow but increasing engagement with disability studies ${ }^{4}$ in MT (Hadley, 2014; Tsiris, 2018), the proposed research is timely in contributing another voice and position to these critical approaches.

\subsection{Therapists' life events shape practice and impact on professional identity}

There is a need to examine therapists' life events and shifting identities as these shape practice and have an impact on the development of professional identity (Goldberg et al., 2014; Hadley, 2001, 2003, 2013b; Rønnestad \& Skovholt, 2003). Aldridge (1993), states that "music therapy is a transpersonal happening and what happens cannot be separated from the person of the therapist" (p.120). Our professional and personal experiences and identities form a lens "that affects how we see and experience the

\footnotetext{
${ }^{4}$ Disability studies operates in a social oppression paradigm which contrasts with medical sociologies underpinning of disability as social deviance (Thomas, 2007).
} 
world and how we think about ourselves and others" (Morella-Pozzi, 2014 p. 176). Therefore, understanding others is inextricably linked to understanding ourselves (Foucault, 1997b; Phillips, 2007). Interestingly, Edwards (2005) notes, that most clinical studies do not scrutinize what the therapist does or include the therapist's experiences/perspectives: they instead focus heavily on the needs and experiences of the client. Likewise, student music therapists tend to focus on the client (Bunt \& Hoskyns, 2002). Whilst studies utilising critical approaches are likely to include therapist positions, Edwards' observation still appears to be the case for many other studies and presentations of practice.

\subsection{Increasing tools that may support reflexivity}

Because arts-based autoethnography is still emerging and there is a paucity of Foucauldian forms of analysis in MT, my research aims to determine what the tools utilised in these methodologies have to offer everyday practice. Due to the inseparableness of the therapist's self from their practice, it may be of benefit to continue to extend our current toolkit that supports a reflexive process. In particular, this includes supporting the integration of personal and professional knowledge, uncovering normative assumptions, and assisting in the reconceptualising practice.

It has been suggested that autoethnography may support reflexivity (Foster, 2013; Hoppes, Hamilton, \& Robinson, 2007; Jones, 2012; McIlveen, Beccaria, du Preez, \& Patton, 2010). Reflexivity is a practice used both within autoethnographic research and therapeutic work that helps us to be aware of our responses to other people and events and to use "this knowledge to inform our actions, communications and understandings" (Etherington, 2004, p. 19). Furthermore, autoethnography offers an applied cultural lens to personal narratives, linking personal understandings with cultural meaning during reflexive thinking.

Arts-based practices and Foucault's work may also further support reflexivity. Because the creative and art-making process is crucial in MT, the use of creative practices as a reflexive tool helps to ensure outcomes are representative of the realities encountered in the therapeutic relationship. This can inform and contribute immensely to our understanding (Baker \& Krout, 2012; McNiff, 1998). As far as I'm aware, Foucault's "critical ontology of ourselves" (Foucault, 1984b, p. 47) has not been used in MT and therefore it is important to see if new knowledge emerges from its use. 
This form of self-analysis offers defamiliarising and reconceptulisation tools that may help identify hidden practices of the self through the investigation of three domains: truth, power, and ethics.

\section{Thesis structure}

Throughout all of the chapters, journal entries and personal accounts-including creative writings-will be in text boxes. This thesis starts with three preparatory chapters. Following this introduction, it explores the literature alongside a composite patient history (chapter 2). The research methodology is then outlined. Chapter 3 considers not only the suitability of the methodology for the research questions, but its compatibility with the therapeutic values, practice and epistemological needs of a music therapist, ultimately assessesing the potential impact of the methodology on practice.

The middle of this thesis encompasses four analysis chapters: "Seeing the unseen: the shadow puppets of disability and illness' (chapter 4), 'Disclosure: to hide or not to hide in the box?" (chapter 5), "Falling in the space between: Oppositional binaries" (chapter 6) and "Finding ladders: Success" (chapter 7). As the analysis chapters primarily look at the first two domains of Foucault's "critical ontology of ourselves" (Foucault, 1984b, p. 47) (truth and power) whilst only touching on ethics, the following section further develops the third domain (ethics) by "creating the self as a work of art" (Foucault, 1984a). This section is split into two parts: part one leads to the development of Post-Ableist Music Therapy (PAMT) (chapter 8), while part two explores my changing subjectivity through illness/disability, music, and research (chapter 9).

The last section brings together the study's findings in discussion with the literature (chapter 10). It concludes with a final chapter that reflects on the research process, providing recommendations and final remarks (chapter 11). Whilst the findings are not immediately generalisable, it is hoped that from directly addressing uncomfortable experiences and topics that others are moved to interrogate their own hidden aspects of practice and uncomfortable silences. 


\section{Chapter 2}

\section{Literature Review: A Patient History}

"Patient files": Photograph of my own patient files from 2007-2018

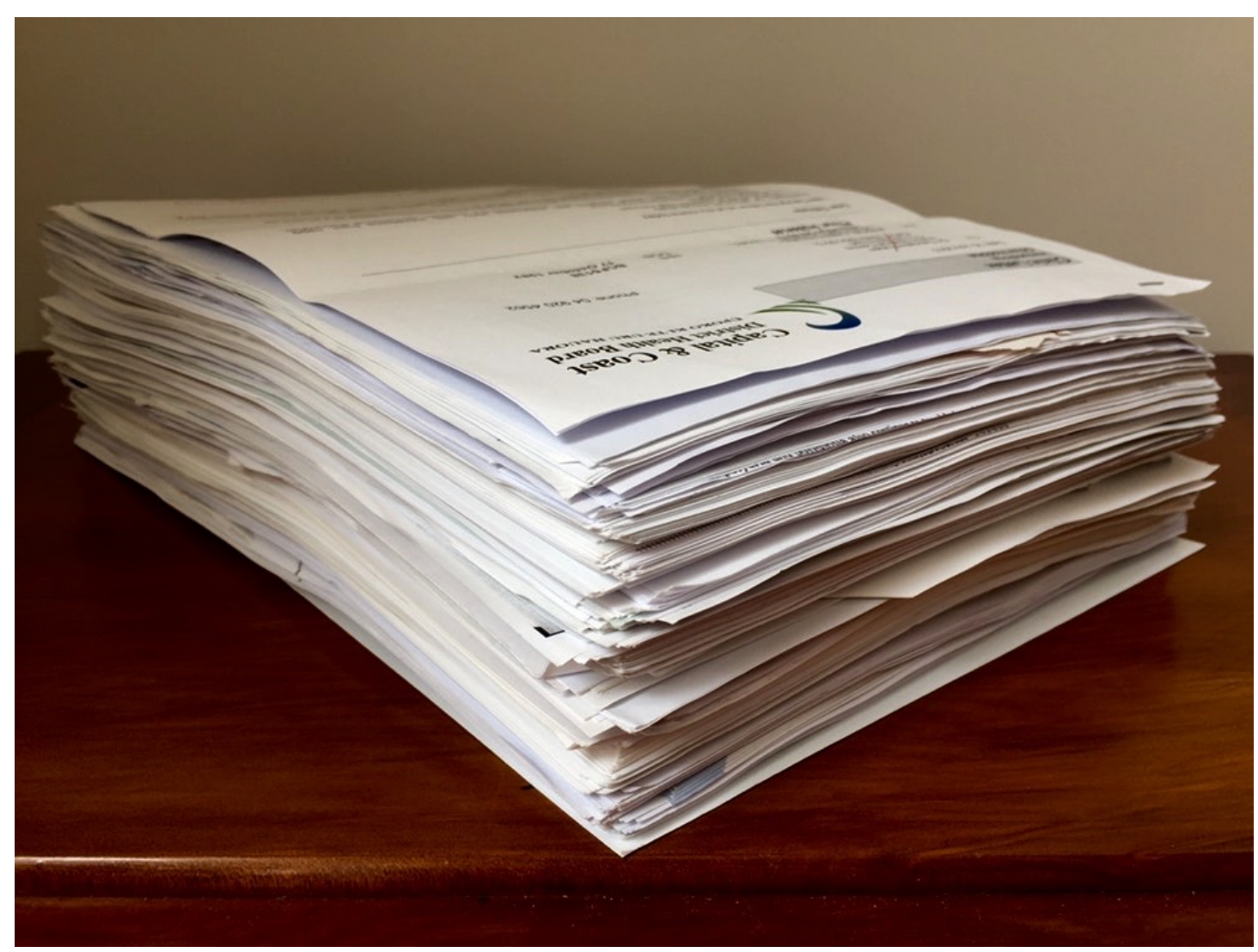

[A large stack of paper on a table] 


\section{Introduction}

Medical consultations always start with the patient's history, and at the beginning of research there is a literature review. They are not too dissimilar: both gather information: look at what has been said: consider key issues: uncover what's missing: discuss a way forward, making recommendations. As I review the contributions of prior scholars, I will present the discussion in the form of a patient history that draws on aspects of my own patient histories. The patient in the history is therefore me (Mrs. Shaw); my own reactions, curiousness, and medical records are conveyed. The fictitious doctor (Dr Zambezi) attempts to answer the questions I have. His character's tone and commentary was created from the statements made in my previous records from multiple doctors.

I hope this novel way will offer the reader a sense of the position from which I first encountered the literature at the beginning of my research: a practitioner-patient (PP) who spent much time encountering the medical world personally as well as working in educational contexts in New Zealand (NZ) that still enculturated medicalised understandings of disability ${ }^{5}$. Presenting it in this way also enacts my reactions and confrontations as a PP from reading the literature. Furthermore, by deliberately presenting some of my pre-research analysis experiences ${ }^{6}$ through a third person (the fictitious doctor) it represents the all too common reality for many people with illness/disability: their voices are often represented indirectly through other people, such as researchers, doctors, family members, therapists: it portrays some of what it feels like to be the subject of reports and the expert gaze of others. Like Rykov (2011), I was concerned that writing the literature review in purely "academic" prose would "fall flat on the page in a manner that was not worthy" (p.4) of the experience of being a PP.

This literature review is relatively concise because there is much discussion of the literature throughout the following chapters. Therefore, this section provides an

\footnotetext{
${ }^{5}$ I acknowledge the work to shift dominant thinking through the discourse of inclusive education. However, IEP goals, processes and role discriptions, for example, were framed by medical model understandings in the majority of schools that I worked at.

${ }^{6}$ I have only drawn on initial thoughts/experiences I had prior to beginning my research analysis as to not preclude in this literature review.
} 
introduction to the literature, and the proceeding chapters extend it. I have taken a broad approach that has included literature from the central discipline of music therapy (MT) as well as related and allied professions. The main area of my search was placed on practitioners who have experienced illness (physical and mental) and/or disability. Literature regarding patient, disability, and practitioner narratives were also included. This seemed appropriate as the study of a PP will contribute to, and is informed by, patient, disability, and practitioner narratives.

Regarding literature pertaining to MT, I searched the last 10 years in Englishspeaking prominent journals: Music Therapy Perspectives, Journal of Music Therapy, British Journal of Music Therapy, Australian Journal of Music Therapy, Canadian Journal of Music Therapy, Nordic Journal of Music therapy, Approaches: An Interdisciplinary Journal of Music Therapy, New Zealand Journal of Music Therapy and Voices: A world Forum for Music Therapy. The review of the literature is structured by first exploring the potential benefits and detractions of being a PP. It ends with exploring the potential support, tools and resources suggested in the literature for PPs.

\section{Patient History: A review of the literature}

Name $^{7}$ : Mrs. Shaw

Problem list $^{\mathbf{8}}$ : Please see the attached report from immunology.

Review: I saw Mrs. Shaw today with her husband. She noted some concerns about her return to work. Our discussion today focused on the three main questions she raised: "Is the return to work appropriate?"; "What concepts and approaches in MT may support PPs in the profession?"; "What tools and resources are indicated in the literature for PPs"?

\footnotetext{
${ }^{7}$ As this patient history is based on my previous patient histories and my thoughts when encountering the literature, I have used my last name to indicate that the patient is me despite the third person reference.

${ }^{8}$ Patient histories in local private and public settings start with a "problem list" which states diagnoses. My last report from immunology at the time of writing stated 11 diagnoses/medical problems. I have deliberately not listed them in this research, as it is not the diagnostic names that capture what it is to embody them, nor do they provide understanding of what they mean to live with. I realise I am already resisting, and veering from, the position I intend to demonstrate by presenting a patient history.
} 


\subsection{Is the return to work appropriate? The double-edged sword}

To answer this question, we explored the potential benefits and detractions of being a PP discussed in the literature.

\subsubsection{Music therapy literature}

Mrs. Shaw may be struggling with her question around returning to work as literature has acknowledged that the implications of practitioner disability on practice is almost absent from the discourse of MT (Rolvsjord, 2014). There may be some isolation experienced from the relative silence. However, I explained to Mrs. Shaw that MT reports/essays (Hadley, 2017; LaCom \& Reed, 2014; Rolvsjord, 2014), research (Hadley, 2001, 2003) and interviews (Bunt, 2004) have noted that people in her position may experience both helpful and negative impacts on their practice.

We first discussed the potential detractions. PPs may experience issues around disclosure (LaCom \& Reed, 2014). Deciding whether to disclose or not can be complicated and challenging. There can be an ongoing negotiation of the position with practice (Bunt, 2004) and disabling barriers in the social and cultural contexts in which Mrs. Shaw may work and study (Hadley, 2017). Hadley (2017) describes how student therapists who have a disability have experienced discriminatory microaggressions in the profession and work place. The microaggressions discussed related to professional exclusion, barriers in obtaining and utilising accommodations, and demeaning work-place interactions.

The small amount of literature pertaining to music therapy PPs demonstrates that Mrs. Shaw may find her position shapes how she comes to view and conduct her practice in positive ways. Hadley's doctoral research explored the life and work of two MT pioneers, Clive Robbins (Hadley, 2003) and Mary Priestley (Hadley, 2001). Clive and Mary had experienced mental illness, disability, and/or mental stressors. Hadley described how these experiences shaped their theoretical approach to practice. For example, Priestley's analytical MT approach developed out of her personal experience with manic-depressive episodes and the influence of her Kleinian analyst (Hadley, 2001). Likewise, Dunn (2009) discusses his parallel journey with a client which details his own experience of mental illness. He examined how this affected the therapeutic relationship and concluded that his illness was not a barrier, but was 
creatively used to support the client. An example of a NZ article drawing on a therapist's experience of illness to inform practice is co-authored by Webster and Rickson (2018). From Webster's personal account, they suggest that when considering the best music for patient listening, preferences and use may change when one is unwell. These examples may explain why Mrs. Shaw voiced that she is "feeling differently" about her work-related tasks. She can feel heartened by the fact that MT practitioners have made significant contributions as PPs.

Authors from other disciplines have also used their personal illness/disability experiences to inform MT. An ethnomusicologist, Schrag (2015), who uses arts practices for healing, found that his experience with Huntington's Disease offered some insight. He suggests that therapists can support people with conditions like Huntington's by using methods that elicit artistic biographies, spark community creativity, and become multiartistic activists. Lewis (2017), also a writer from another discipline, found insight into how music affords recovery from her experience of child abuse.

Whilst unsettling, the PP position may bring to the fore problematic power dynamics within the therapeutic relationship, which if recognised may have a positive impact on critical thinking in practice. For example, therapists may be confronted with how the dis-abled aspects of their self might destabilise the perception of "expert therapist" and highlight issues of the patient-therapist divide. The privilege inherent in therapists having more choice over whether to disclose than the client may become a more apparent issue (LaCom \& Reed, 2014; Rolvsjord, 2014).

\subsubsection{Related professions}

Music therapy PPs may draw similarities with PPs from other related professions: psychotherapy, social work, nursing, medicine, and occupational therapy (OT). A small body of research, within the corresponding disciplines, illuminates the potential challenges and benefits of experiencing illness/disability as a practitioner. Alongside this research, literature consisting of reports and essays also discuss the experiences of PPs. We drew on this literature to assist us in determining whether Mrs. Shaw's return to work might be appropriate at this point in time. 
In psychotherapy, there has been a rapid increase in articles written on the previously taboo subject: countertransference, self-disclosure, and the positive and negative impacts were topics frequently addressed. Dewald's (1994) essay discusses countertransference issues for PPs. He suggests that a potentially valuable opportunity can be lost if the therapist and patient avoid paying attention to countertransference issues. Leon's (2004) report discusses how he navigated and reflected on countertransference and therapeutic processes arising after sharing the same diagnosis as the therapy group he facilitated for Parkinson's patients. Psychotherapist Morrison (1997) also gives a personal account of the countertransference she experienced in her work related to her cancer diagnosis. She explores the decision-making around selfdisclosure and the clinical implications: disclosing when helpful for patients and refraining when not.

Other positive and negative implications were discussed in two studies regarding therapists with histories of eating disorders who were working with the same client population (Johnston, Smethurst, \& Gowers, 2005; Rance, Moller, \& Douglas, 2010). The potential dangers outlined for the therapists were over-identification, involvement, and enmeshment. The potential benefits discussed were increased empathy, understanding, resilience, and enhancement of the therapeutic relationship.

Mrs. Shaw pressed for further information. We may need to consider that, as research in other disciplines suggests, she may struggle to manage the integration of her professional and personal knowledge and needs. Goldberg et al (2014) investigated social work students with psychiatric difficulties. The data gathered from interviews uncovered a four-stage process, where the student moved from exploring and questioning the possibility of a patient being a therapist through to the final stage, where they were able to integrate their patient and therapist selves, allowing them to live together and enrich each other. It showed that there were additional challenges and dilemmas for students with psychiatric difficulty, such as having concerns that the patient part of themselves might impact negatively on their client. Participants felt that they had to navigate these challenges and the process of integrating their professional knowledge and personal patient experiences silently, alone, and without guidance. Mrs. Shaw felt particularly disconcerted when I discussed the findings in Neal-Boylan's (2012) interpretive naturalistic inquiry, which showed that it was rare for even qualified health professionals who had a disability to ask for 
accommodations and support for fear of being seen as trouble-makers or incompetent, demonstrating the struggle in integrating personal and professional needs.

Mrs. Shaw may potentially experience internalised and externalised stigma that may further hinder such integration. Like a lot of the issues brought up in today's discussion, this is outside of my area of expertise. I am aware of a nursing study in the form of a collective autoethnography, by Kidd \& Finlayson (2010), that examined practitioners who had experienced mental illness and identified similar themes of interest. It was felt that therapists' patient experiences helped to increase their ability to understand patients' perspectives and empathise with them, resulting in improved patient outcomes. However, all nurses in the study experienced internalised and/or externalised stigma. This resulted in the perception of having to choose between silence as a nurse and receiving care as a patient. Similarly, Korzon's (2012) study indicated systemic discrimination against nurses with impairments, which contributed to the participants negotiating the identity of disability largely in isolation.

Furthermore, workplace environments that operated on an assumption of nondisability created tension around issues of disclosure and non-disclosure, which were complicated by internalised and externalised stigma. As a side note, given the invisibility of Mrs. Shaw's condition, she may be assumed to be non-disabled. However, there is a resultant disability from her impaired function. The results from the tests enclosed lay outside of normal range, indicating "moderate to severe" abnormalities. Some irregularities have "failed medical treatment"10 11.

There is no cure for Mrs. Shaw's condition. However, to avoid some of the potential negative impacts of her illness on her work, as described above, we reviewed and considered further pharmaceutical interventions. Mrs. Shaw felt that the medications given by previous specialists have been partially helpful. She has tried different beta blockers (propranalol, atenalol, metoprolol), calcium channel blockers (Nifedipine, IV Iloprost infusions), sinus node inhibitor (Ivabradine), oral cholinesterase inhibitor (Pyridostigmine), DMARD’s (hydroxychloroquine), antimuscarinics (Solifenacin), Steroids (IV Methylprednisolone, Hyrodcortisone, Prednisolone, Fludrocortisone), non-steroid anti-inflammatories (Naproxen), as well as a slew of anti-nausea

\footnotetext{
${ }^{9}$ Wording from autonomic function tests.

${ }^{10}$ Quote from one of my patient histories by a surgeon.

${ }^{11}$ Doctors' define and label disability in medical terms.
} 
medications, proton-pump inhibitors, antihistamines, analgesics, and sedatives. Her current treatment (please see overleaf for details) is providing partial relief from symptoms, and I'm investigating IVIG (intravenous immunoglobulin) access for further symptomatic treatment. I consider that we have explored all possibilities at this point in time. She is of childbearing age and has declined trialing some treatments that may impact on fertility and pregnancy. From my end, there is not much more I can offer that would dampen symptoms to a level where she would not need to consider the impact of her impairment on practice.

Given this, the potential impact her condition may have (positive or negative) on her work was further considered. Being a PP may contribute to questioning professional culture and values. In Frank's (2004) chapter titled, "physicians generosity", doctors who had been marginalised in some way developed a questioning of received values. The cultural values of a profession clashing with the values of a therapist with an impairment, is also echoed in the OT literature. Chacala et al., (2013), found four major themes: a clash in the cultural values of the profession with disability culture; negative attitudes creating barriers to work; having to take responsibility for the disabled-nondisabled cultural divide exacerbating inequity; and disability offering advantages for practice rather than reducing effectiveness.

Continuing with OT literature, Bevan (2013) found that the majority of barriers for PPs were attitudinal rather than environmental or organisational. Despite these barriers, the studies reviewed in OT consistently mentioned that impairment could be an asset to their practice. Velde's (1999) phenomenological study elicited that PPs approach their practice from a unique perspective, consider themselves to have unique skills, and have developed strategies to cope with the experience of disability.

\subsubsection{The "wounded healer"}

We also considered the "wounded healer" literature as it similarly discusses whether the position is of help or hindrance to practice. The concept is discussed in MT literature (Austin, 2002; Bunt, 2004; Pavlicevic, 1997; Rolvsjord, 2014) and within other disciplines (Zerubavel \& O'Dougherty-Wright, 2012). The term refers to a healer who has an inner "woundedness", like illness or trauma, which is thought to contribute to the capacity to support others (Jackson, 2001). Wounded healers use their personal experience constructively to form attitudes, practices, increase empathy 
or develop transformative qualities that are used usefully in therapeutic endeavours (Jackson, 2001).

Wounded healers have been historically written about in many contexts, such as in shamanism, in pastoral care, and in the myth of Chiron the centaur (Jackson, 2001). The Jungian tradition suggests that all healers are wounded, but that "wounded" and "healer" are conceived of as a duality rather than a dichotomy (Pavlicevic, 1997; Zerubavel \& O’Dougherty-Wright, 2012). If they are seen as dichotomous it can negatively influence the therapeutic process, firstly by the therapist denying their own suffering (and being unable to access their experiences to empathise with the client), and secondly it may result in a lack of acknowledgement that the client has their own healing powers (thereby stifling encouragement of the client's ability) (Pavlicevic, 1997; Zerubavel \& O’Dougherty-Wright, 2012). Activating and internally dialoguing both "wounded" and "healer" at the same time is considered advantageous. This idea links with the previous literature about the need for PPs to integrate professional and personal knowledge, which Mrs. Shaw admits she is working on.

Whilst Austin (2002) notes the helpful aspects of being a wounded healer that are echoed in literature, she also discusses the particular challenges for MT wounded healers. The intimacy of creating music together can be challenging because unconscious aspects for both therapist and client can be easily accessed through music. The need to be well acquainted with one's own feelings in order to differentiate client's feelings is intensified when the therapist is a wounded healer. She suggests that wounded healers like herself should attend therapy, supervision and peer support, in order to guard against potential vicarious trauma and countertransferential issues.

Zerubavel et al (2012) discussed the tendency in the psychology profession to silence, reject, or stigmatise the wounded healer. This is thought to stem from social stigma and wariness about the healer's stability. I could refer Mrs. Shaw for more testing to further ascertain and demonstrate her stability, if it would help support her working and provide others with reassurance. Paradoxically, it is thought that being open and accepting of woundedness helps us to become more human and more real as therapists and less likely to cause harm (Pavlicevic, 1997; Zerubavel \& O'DoughertyWright, 2012). 


\subsubsection{Summary of the appropriateness of the return to work}

Literature across disciplines suggests that being a PP/wounded healer may enhance practice: as a catalyst in shaping and developing one's practice; increasing the questioning of professional cultures and values (supporting critical thinking); increasing empathy and resilience; and supporting the understanding of client's perspectives. Ultimately, it may enhance the therapeutic relationship. However, the literature also suggests detractions for PPs. They may experience microagressions, stigma, discrimination, and barriers to seeking accommodations. It may be challenging to negotiate the position and to integrate professional and personal knowledge, resulting in negative impacts on practice. Over-identification, involvement, and enmeshment can occur. The silence around PPs and lack of attention creates isolation, shame and conflict about disclosing. Lastly, professional cultural values and PP values may clash. To complicate matters, the literature suggests that disclosing one's illness/disability can have positive and negative implications for clients, and that countertransference occurring from sharing a similar position can also be a help or hindrance.

To answer Mrs. Shaw's question, return to work may be appropriate. However, given the potential detractions discussed, it appears that this will need to be well-managed to mitigate some of the negative impacts. Tools and resources will need to be put in place to sustain her return to practice.

\subsection{What concepts and approaches in MT can be found in the literature that may support PPs within the profession?}

We then discussed which concepts and approaches in the MT literature might be supportive or less supportive for Mrs. Shaw. Aigen (2014) outlines three stages in MT theory development. The first stage includes theories imported from psychology (psychotherapy and behavioural learning) and the second stage involves treatment models of practice. Medical and psychological models have been considered a grand narrative in MT (Rolvsjord, 2010). Within these, the medical model of disability, which understands disability as a personal deficit or a deviation from documented norms, has dominated: the "problem" is considered to reside in the individual, caused by disease or injury (Mitra, 2006). Derived from this is the idea that diseases and their consequences are adverse, and require efforts to reduce symptoms and eliminate 
disease (Kielhofner, 2005). Mrs. Shaw noted during our consultation, that the recent controversy over the movie Me Before You (2016) underscores the extent to which many people still accept that a life with a disability is a personal tragedy not worth living. Mrs. Shaw expressed that she finds it challenging to read MT literature that strongly utilises medical discourses. Much literature describes disability as deficit (Gooding, 2011; Kwak \& Kim, 2013; LaGasse \& Hardy, 2013; Raglio, Traficante, \& Oasi, 2011) and aims to "treat" children with autism (Lim, 2010b; Raglio et al., 2011) and intellectual disability (Hooper, Wigram, Carson, \& Lindsay, 2008). In this literature there is an overemphasis of need for MT and a tendency to list all the ways the clients were lacking. Reading this literature makes Mrs. Shaw sometimes feel that as a PP she is sorely lacking with multiple deficits and only in need of treatment. This is not surprising given that a research study from nursing found the medical model to be a barrier for PPs (Korzon, 2012).

Although the medical model has been a dominant discourse in MT, music therapists have been challenging the appropriateness of it since the mid 1960s (Aigen, 2014), and practices continue to diversify. Some contemporary approaches associated with the third stage in MT theory development (Aigen, 2014) offer more balanced and celebratory writings of difference. They include a plethora of models and forms, such as interdisciplinary and indigenous MT theories. Examples of some of the third-wave orientations, which seek to not pathologise the individual and/or support social justice, include: Kenny’s “field of play” (2006); aesthetic MT (C. Lee, 2003); community music therapy (Ansdell, 2002; Pavlicevic, 1997); resource oriented MT (Rolvsjord, 2010); music-centered MT (Aigen, 2005); anti-oppressive MT (Baines, 2013); feminist MT (Hadley, 2006); culture-centered MT (Stige, 2002). There has been a general movement in MT practice away from the medical model that grounded much MT practice in the $20^{\text {th }}$ century towards practices that foster social change, value diversity, and approach disability from other perspectives (Rickson, 2014d). Previously marginalised perspectives are increasingly included and utilised to inform theoretical thinking/practice. For example, the queer MT model by Bain, Grzanka, \& Crowe (2016), generated much positive attention for its future implications in MT (Boggan et al., 2017). MT journals have become increasingly inclusive with some seeking to increase diversity. For example, the Voices editorial team seek to represent a full range of positions and nurture a critical edge focusing on social justice and 
cultural issues (McFerran, 2017a). MT is becoming increasingly diverse, providing a supportive environment/space for therapists to contribute to theoretical knowledge from personal lenses of disability.

MT practices have diversified with the inclusion of alternative understandings of disability, reducing the barriers that the dominant medical model may pose for PPs. The social model of disability understands disability as a social construct: instead of being attributed to the individual, it is created by social environments and therefore requires social change (Mitra, 2006; Oliver, 1996; Shakespeare, 2013). The term was coined by Michael Oliver in 1983 (Thomas, 2007). Pfeiffer (2001) outlined nine versions of the social model of disability, one of the most prominent being the social model of the United Kingdom which understands disability as social oppression (Oliver, 1996). This form developed from the ideas of the organisation, Union of the Physically Impaired Against Segregation. Gross (2018) gives practical suggestions to implementing the social model of disability in MT practice, such as being aware of hierarchies.

Mrs. Shaw became quite excited when she shared with me the interdisciplinary special issue in Voices on MT and disability studies (2014, vol. 14, n. 3). Disability studies operates in a social oppression paradigm where impairment is understood not as a personal lack or limitation, but as a unique way of being situated in the world (Kielhofner, 2005). This contrasts with medical sociologies' underpinning of disability as social deviance (Thomas, 2007). In MT, "cure narratives" that are aligned with the medical model differ from disability studies' absence of these (Bassler, 2014). In this special issue of Voices, LaCom and Reed (2014) discuss the instability of disability and outline Reed's questioning of her understandings of disability as a disabled MT student. Outside this issue, there are a small number of articles, chapters, and presentations that explore or include this interdisciplinary exchange and dialogue between disability studies and MT (Gross, 2018; Hadley, 2017; Hadley \& Thomas, 2018; Metell \& Stige, 2015; Tsiris, 2013, 2018). The term PP may not be as appropriate within the context of disability studies as often disability is seen as part of identity within this literature, not as something to be fixed through being a patient. A disability studies perspective would consider whether the contexts Mrs. Shaw works in have any disabling barriers that impact her work, potentially offering some support. 
Literature relating to social models of disability seem to highlight that disability can help therapists in their work with clients, and any hindrances come from unhelpful/disablist attitudes or working environments (Bevan, 2013; Hadley, 2017). However, Rolvsjord (2014) raises an important caution to viewing only the therapist's disability as a potential positive generative component whilst not extending this to client's disabilities. She states that "we tend to view such vulnerabilities in the therapist as strengths, by for example implying that these vulnerabilities make empathy possible. While moving back to the client...strengths... are easily ignored or considered of less relevance regarding the therapeutic process" (Rolvsjord, 2014). It would appear that in much literature, the disabilities/illnesses of PPs and disabled practitioners are given a different status to that of the clients.

Continuing with alternative models of disability, the bio-psycho-social model incorporates both social and medical understandings. This model understands functioning and disability as a dynamic interaction between health conditions and contextual factors, both personal and environmental. The International Classification of Functioning (ICF), adopted this as the conceptual framework for the world report on disability (World Health Organisation, 2011). It posits that disability has its genesis in a health condition, which gives rise to impairments that limit activity within contextual factors. An example of a MT paper that draws on aspects of this model is McDermott, Orrell, \& Ridder's (2014), which explored the importance of music for people with dementia.

There are other definitions of disability in the wider literature, such as the capabilities approach (Mitra, 2006), the affirmation model (Swain \& French, 2000), and the ethnographic model of disability (Bakan, 2015). In MT, Rickson (2014) used the affirmative model of disability, which doesn't deny negative aspects of impairment but recognises that it is not always undesirable, even valuable, in her research with young people with intellectual disability. Additionally, Straus (2011), outlines four ways that western thought has historically understood disability: 1) as a permanent affliction, 2) as a divine inspiration, 3) as an individual pathological deficit, 4) as a social, personal, cultural identity affirmatively claimed. In short, disability has been subject to many definitions within different disciplines. Accordingly, the multiple definitions of disability may lend to different understandings of PPs/disabled therapists and have varying implications for them. 


\subsubsection{Summary of the concepts and approaches in MT that may support PPs}

From the literature reviewed, the historically dominant medical model of disability may be a barrier for PPs, due to its individualistic focus and pathologisation. Alternative understandings of disability are explored in the MT literature and offer alternative positions for PPs. Many critical approaches may support PPs in the profession due to their contribution towards diversifying practice and social justice focus. They offer potential in addressing some of the issues facing PPs. For example, the dissonance between the profession's values and that of the PP may lessen within critical orientations due to the reduction of pathologisation and discrimination through the social justice focus. The increasing diversity in contemporary practice suggests greater professional inclusivity. Perhaps there is less need for therapists, like Mrs. Shaw, to remain silent. The diversification of practice could be further supported by hearing from more, and making visible the experiences of, PPs. Literature from OT and psychology is encouraging in this regard (Neville-Jan, 2003; Sollod, 2002).

\subsection{What tools and resources are indicated in the literature for therapists who experience illness/disability?}

As pharmaceutical treatment has not resulted in full resolution of symptoms, Mrs. Shaw might be interested in the tools, resources, and techniques that PPs/wounded healers across disciplines have used to navigate their work. It appears that writing and talking about her experience as a PP may help to externalise the problem, and that spending time reading other people's experiences of illness may be beneficial. Consulting with colleagues about potential issues that arise in practice is also considered a useful strategy for PPs in the literature (Dewald, 1994; Zerubavel \& O’Dougherty-Wright, 2012).

Writing was the most discussed tool. A psychotherapy article introduced the technique of externalising the problem through writing as a useful strategy for PPs. From her experience with lupus, Maggio (2007), discussed how writing allows one to move the issue outside the individual allowing them to reflect on their struggles from different perspectives. Writing stories about one's practice may help develop critical thinking and challenge assumptions, assisting in deep thinking about patients (Sharpiro, 2012). The narratives written may also help healthcare professionals to 
process and explore complex emotions in themselves and others, and assist in stress management, self-awareness and self-care (Roscoe, 2012).

Narrative medicine has led to a resurgence of interest in narrative writing, for not only the benefit of patients but for physicians as well (Sharpiro, 2012). Narrative medicine means practicing medicine with "narrative competence" with the intent to bridge the patient-health professional divide (Charon, 2001, p. 1897). A tool for developing narrative competence is writing reflective texts about one's practice and reading patient narratives (Charon, 2001). PPs can write about both experiences of being a patient and practitioner and therefore contribute to the body of narrative medicine literature from both sides.

Some patients have written personal illness narratives as a way of expressing their experience, for political activism, and to communicate something of what it is like to be a patient (Aronson, 2000; Easton \& Atkin, 2011). Popular examples include JeanDominiques Bauby's memoir The Diving Bell and the Butterfly (1997) and John Diamond's C: Because Cowards Get Cancer Too (1998). Autopathography is a term used for accounts of being a patient (Aronson, 2000). During the last twenty years there has been an increase of autopathographies written. The benefit of these can be in raising awareness in the general public and enabling doctors to gain access to the experience vicariously (Aronson, 2000). Within research literature there are patient narratives in the form of autoethnographies regarding various types of illness, such as chronic pain (Birk, 2013; Neville-Jan, 2004; White \& Seibold, 2008), dystonia (C. Smith, 2012), ulcerative colitis (Moore, 2013), thyrotoxicosis (Ettorre, 2005), acquired brain injury (Claire Smith, 2005), kidney failure (Richards, 2008) and mental health (Brooks, 2011; Kidd \& Finlayson, 2010). One autoethnographer reflected on the transformative learning tools of the creative arts for those with chronic illness, in particular using music for processing (Storer, 2014).

Mrs. Shaw may be interested in finding local support. A Victoria University of Wellington $\mathrm{PhD}$ researcher wrote a personal narrative on living with chronic pain, alongside a study of three writers who had lived with and had written about pain (de Montalk, 2013). At the end of her thesis she asks, "If we cannot feel another's pain, is it possible to imagine or understand it?" (p.306) She felt that there was a possibility that we are hard-wired not to accept the pain or woundedness of others, but that 
sharing stories of pain can provide empathetic disclosure between patients. Such sharing and resonant understanding can help decrease feelings of isolation (Defenbaugh, 2008) and support people in coming to terms with and recognising their experience (de Montalk, 2013). Mrs. Shaw pointed out that, interestingly, much literature that explores patient experiences often overlooks this direct benefit patients can get from reading it. For example, the book, Understanding and Using Health Experiences: Improving Patient Care edited by Ziebland, Coulter et al (2013), is tailored to healthcare professionals and does not mention the benefits of patients accessing and reading it.

If Mrs. Shaw does decide to write her stories as a tool to navigate the position, there are possible detractions to be aware of. Literature indicates that patients may encounter problems in writing their stories as illness may trouble one's ability to share their story coherently, intelligibly, and therein exacerbate their suffering (Frank, 1995). Because of this, some authors and researchers write their narratives retrospectively (Birk, 2013; Neville-Jan, 2004). Furthermore, Richards (2008) explains that illness stories are often intimate and may leave the writer vulnerable. Different models of disability suggest differing understandings about where the tools and resources should be targeted. A medical understanding would target the individual rather than the social contexts that would take focus within social models. I have highlighted the tools and resources that Mrs. Shaw might like to try, such as writing and reading, but I am less sure about how to resource her work contexts. However, Korzon (2012) suggests the following ways to support inclusive environments for nurses with disabilities: foster positive attitudes in embracing diversity through challenging medicalised understandings of disability; provide professional development for practitioners in examining cultural scripts of disability; focus on advocacy and utilise positive recruitment strategies. Hadley (2017) suggests that to effectively deal to the microagressions disabled music therapists and clients face, practitioners need to develop an anti-oppressive practice, which she draws from Baines (2013). 


\section{Summary of literature review}

Literature suggests that there may be a lot for Mrs. Shaw to navigate and negotiate in her return to work. PPs can experience stigma, discrimination, and microagressions across a variety of professions including and relating to MT. They do not always get the resources they need to do their work. Their position can have both positive and negative implications for their practice, making the position something of a doubleedged sword.

Given these found complexities, tools, resources, and orientations that may support Mrs. Shaw were considered. Overall there was a paucity of MT research relating to therapists who experience illness/disability and further attention is required. In the context of NZ, apart from one article (Webster \& Rickson, 2018), no other research was found that explored this area, further contributing to the silence of such voices in this context.

Integration of both personal experiences and professional perspectives was viewed as helpful and important in the literature for PPs. Tools that may help support this process for PPs were: writing their patient and practitioner narratives; reading other people's experiences of illness/disability; and discussion with colleagues/supervisors. However, the tools and resources that were tailored specifically to PPs were limited overall, and more recommendations for the social spaces they practice in are needed.

Autoethnography, creativity as a medium for process, and Foucauldian tools need further consideration as potential tools for MT PPs. From the papers reviewed, no MT study used a poststructural or Foucauldian lens for analysis within the framework of an arts-based autoethnography. Therefore, a study that draws on this methodology and assesses its relevant tools for practice is uniquely positioned to add to new knowledge.

The literature indicates that MT practice is becoming increasingly diverse with the incorporation of multiple models of disability and critical approaches that address social justice. Thus, positioning it well to include and support the voices of PPs/disabled therapists. With this in mind, it seems timely for Mrs. Shaw to 
contribute to theory development by proceeding with the examination of her narratives of illness/disability alongside her practice, to see if further meanings and practices can be uncovered.

I wish Mrs. Shaw all the best in making decisions around whether to continue her MT work. I offer support for her proposed study and would be happy to review with Mrs. Shaw at a later time.

Kind regards,

Dr Zambezi 


\section{Chapter 3}

\section{Methodology}

"New growth within surrounding structures": Photograph taken in Wellington CBD

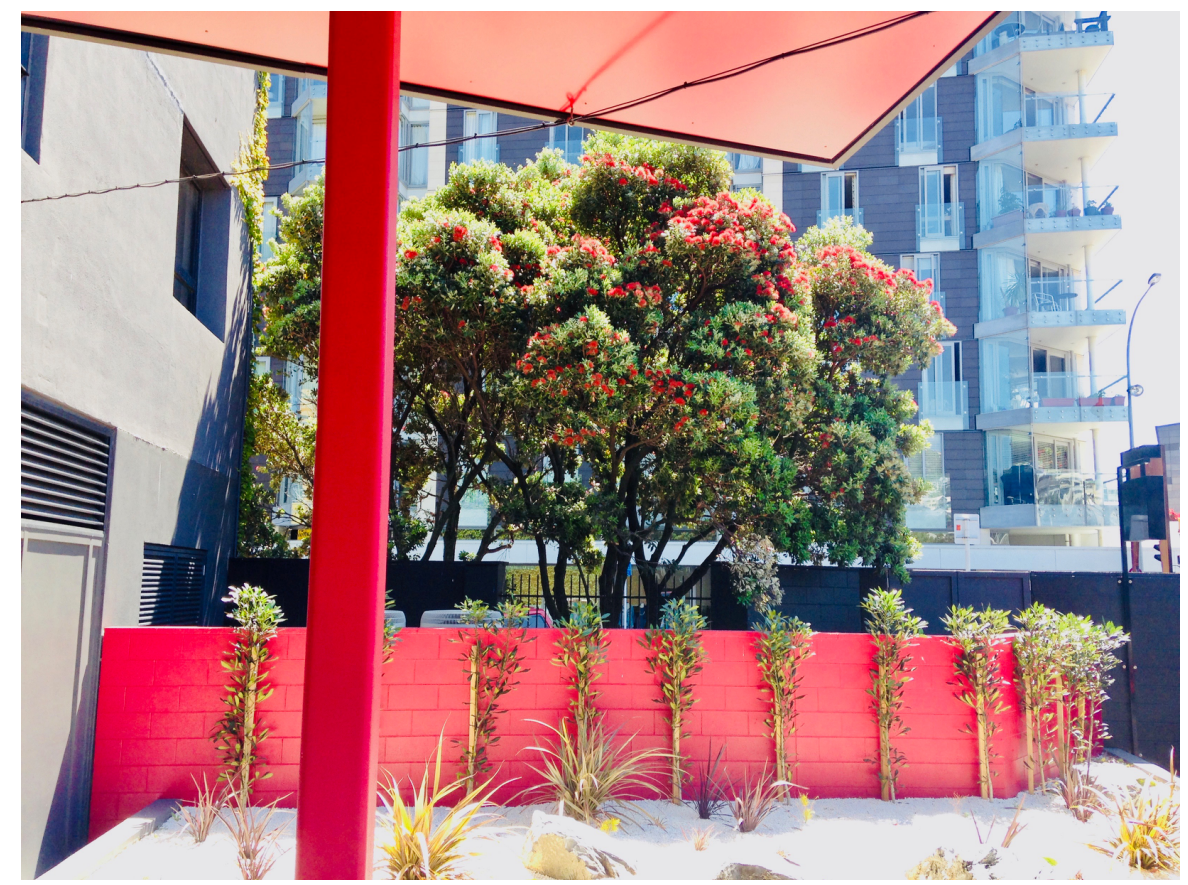

[A flowering pōhutukawa tree and new plants growing amongst surrounding tall building structures]. 


\section{Introduction}

Outlined in this chapter are the research approach, the theoretical underpinnings, the data gathered, the analysis taken and ethical considerations. The chapter ends with a prelude to the analysis chapters that follow. When making methodological decisions, the suitability of the method for the research questions was not the only important consideration. Also important was the compatibility of the methodology with my therapeutic values, practice and epistemological needs as a music therapist and its potential impact on my practice. Having an awareness of the paradigmatic context allowed me to evaluate the particular political and worldviews my methodology would promote and the consequences of these (Edwards, 2005a; Peile, 1988). As a research question directly addresses how conducting autoethnographic research may impact on practice, it seemed important to discuss the pre-existing literature regarding this to assess its potential. Literature discussed in this chapter includes autoethnography and arts-based research from music therapy (MT) as well as other related professions.

This chapter will discuss the approach of a poststructurally-informed, art-based autoethnography. I postulate that this approach supports my research questions and complements and benefits practice by elevating attention to the contextual space of therapy, fostering the understanding and use of the therapist's self, and supporting ways of knowing and skills that are essential to practice. Such outcomes are important because MT cannot separate itself from culture (Pavlicevic \& Ansdell, 2004; Stige, 2005), therapists cannot separate themselves from the work (Aldridge, 1993) and there is an iterative relationship between practice and research in which they both influence each other (Hoskyns, 2013).

Throughout this chapter, I interweave literature discussions with reflections on personal experiences that have shaped how I engage with and understand the methodology. As "we cannot separate ourselves from what we do" (S. Wall, 2006, pp. 09-10), by taking these steps I have made the self that has guided these methodological choices visible. 


\section{Research approach}

\subsection{Form of autoethnography}

I used an arts-based autoethnographic approach combined with a poststructural form of analysis. Firstly, I will discuss the autoethnographic aspect. Autoethnography is "an approach to research and writing that seeks to describe and analyse (graphy) personal experience (auto) in order to understand cultural experience (ethno)" (Ellis, Adams, \& Bochner, 2011, p. 1). It is a contemporary qualitative research method within the discipline of ethnography (Grant, Short, \& Turner, 2013). Chang (2008) argues that autoethnography would fall short of being an auto-ethno-graphy if any of the following are deficient: ethnographical in its methodological orientation, cultural in its interpretative orientation, and autobiographical in its content orientation.

Multiple historical developments paved the way for the development of autoethnography. There was an increasing need to resist colonialist and sterile research, contention around the authoritative voice in ethnographic research, recognition that the researcher isn't neutral, the turn of narrative based research in the social sciences, whilst at the same time feminist researchers and disability studies were seeking to extend and accommodate different ways of knowing in research (Ellis et al., 2011; Grant et al., 2013). Scholars turned to autoethnography because "they wanted to concentrate on ways of producing meaningful research that would sensitise readers to issues of identity politics, to experiences shrouded in silence, and to forms of representation that deepen our capacity to empathise with people who are different from us" (Ellis et al., 2011, p. 2; Ellis \& Bochner, 2000).

It is difficult to precisely define autoethnography due to its myriad of forms, approaches and creative representations (Boylorn \& Orbe, 2014b). Some autoethnographies place more weight on the personal narrative and others are weighted more heavily on theoretical discussion (Chang, 2008). However, all autoethnographies are concerned with connecting personal experience to cultural meanings. This social/cultural aspect is important, as MT always takes place in context within our social-cultural life. Therefore, the cultural gaze is not an optional add-on (Pavlicevic \& Ansdell, 2004; Stige, 2005). Autoethnography may further develop a practitioner's awareness of social/cultural factors that influence and are 
influenced by practice. For example, Foster (2013) found that writing an autoethnography facilitated family medicine physicians to cast a critical lens on the culture of medicine. Autoethnography was effective in revealing the "hidden curriculum" of medicine, which refers to the enculturation and automatic nature of what is passed on to new medical students (Foster, 2013).

Whilst there are many different approaches to autoethnography, in developing my methodology I found it helpful to draw on critical, interpretive, and evocative autoethnography. Boylorn and Orbe (2014b) discuss "critical autoethnography" that shares similarities with the conception of critical ethnography. This method uses features of critical theory. Whilst my research does not use critical theory, the features of examining power arrangements and social conditions and challenging these were relevant.

Denzin (2014) describes "interpretative autoethnography", a critical performative practice in which the researcher moves back and forth in time. Interrogation of the cultural and historical conditions that created past experience and events are examined and interpretations of these move the subject/author forward towards action (Denzin, 2001, 2014). There is a social justice agenda to such an inquiry (Denzin, 2014). The data analysis approach taken is similar to the critical performative practice of interpretative autoethnography.

A poststructural lens is used in my research and no other participants are included beyond myself. Due to this, Anderson's (2006) analytic approach to autoethnography did not seem suitable. Analytic autoethnography is more aligned with traditional ethnographic practices than what he termed as "evocative autoethnography", which is often associated with poststructuralism and postmodernism. The poststructural aspect also differs from other music therapy autoethnographies, which draw on phenomenological frameworks (Freeman, 2018; Woodward, 2015), constructivist (Baines, 2014), and interpretivist approaches (Lipski, 2014).

\subsection{Representational features of autoethnography}

This evocative aspect is one of many beneficial representational features autoethnography offers (Grant et al., 2013). Issues of representation were important 
when considering the methodology, as I was aware of the problems I encountered in research regarding my own conditions.

I tried to find my experience in research reports regarding living with chronic illness. I searched because, "take these pills, these pills, and these pills" was insufficient information for how to actually live with the challenges that the pills didn't take away. But the research I found reflected back a different image than the one I lived: An unreflective mirror, showing rationalistic displays of neat findings in contained categories and tables, un-emotive, and disembodied. This being the opposite of illness, where my body screamed to be felt, and seeped its way through the walls of life. Finance, career, relationships, and hobbies all jumbled together in a mess as the walls caved in. I swam in a muddle of mixed conflicting emotions: A sense of joy and pride for managing to walk to the end of my driveway, tinted with sadness and head hanging shame that I only walked to the end of my driveway. Conventional representation strategies of research were unable to convey the depth, complexity, messiness, and contradictions that I had come to know in living the experience of chronic illness.

Four representational features and the benefits of these will be discussed. Autoethnographic research is often:

- Evocative

- Creative

- Complex

- Non-reductionist

Evocative writing in autoethnographies is used to draw the reader closer, instead of creating distance (McIlveen et al., 2010). This quality is thought to help vocational psychologists reduce "othering" in their work and increase sensitivity and empathy (McIlveen et al., 2010). Presenting autoethnographies in creative ways, such as poetry and paintings, can capture the depth of the human experience and bring the reader closer to the experience being studied (Gallardo, Furman, \& Kulkarni, 2009). Creative depictions can reach people on a visceral level whilst conveying the visual, intellectual, emotional, and bodily qualities of the studied experience (Etherington, 
2004). Therefore, art-based representations have the ability to more accurately describe participants' experiences (Rykov, 2011). MT is often hard to convey due to difficulty in using words to explain the non-verbal nature of music making and some experiences (Aldridge, 1993). Autoethnographers seek to present such complexities of experience. They challenge the notion that research should lead to clear, tidy conclusions. Instead they present phenomena as they are-messy, complicated, and confusing (McGraw Hunt, 2016; Spry, 2012).

Along with being evocative, creative, and complex, another benefit of autoethnography is its contextual non-reductionist representation. Grant (2011) highlights that reductionist representation of research that is de-contextualised can lead to people being treated in a corresponding reductionist way, giving the illusion of a simplistic linear trajectory of therapy. Representational practices in autoethnography that embrace contextual, holistic presentations may benefit one's practice: the way clients are described in texts often effects how they are perceived and treated (Grant, 2011).

Like Inkle (2010), I am aware of the multifaceted implications and responsibilities of representation in research. Personally, I can recount many examples of being treated in a distanced and mechanical way as a patient that resembled the privileged statistical and reductionist methods in medical research.

The sharp object, that didn't exist, moved through my skull as soon as I was upright. By day 5, tears fell as my husband lifted me. They said such severe pain was really rare, only a mild headache was to be expected. "It is rare", said not one, not two, not three, but many. For some, statistics filled the air with doubt and closed their ears to my voice. My narrative of agony was indeed correct when it took an epidural blood patch to fix the spinal leak that the lumber puncture had created. I was very unlucky said not one, not two, not three, but many. I didn't feel unlucky before entering the hospital, I felt unlucky when I left.

But I am like the other snowflakes falling from the sky moulded on my journey. My patterns throw off light in a manner like no other, for we all fall differently. 
Counterbalancing reductionist research with alternative ways of representing experiences of illness/disability felt important because representation in research can construct and constitute the real experience in therapy (Grant, 2011). Therefore, autoethnography may positively affect the social practice of therapy: it responds to the representational issues people with illness/disability face by embracing complex, creative, contextualised and evocative ways of presenting research.

\subsection{Arts-based autoethnography}

I used an arts-based form of autoethnography. The various forms autoethnographies take demonstrates that "not all autoethnographers think alike" (Ellis \& Bochner, 2006, p. 435) and that there is scope for the way this method can be framed.

Autoethnographies take a variety of representational forms (Ellis, 2004; Ellis \& Adams, 2014; Ellis \& Bochner, 2006), such as poetic (Gallardo et al., 2009) collaborative autoethnography (Chang, Ngunijiri, \& Hernandez, 2013), and visual art (Averett \& Soper, 2011; Etherington, 2004). Although autoethnographies often have aesthetic sensibilities, the strong presence of arts-based practices in my research justified its conception as an arts-based autoethnography. The inclusion of arts-based practices may help decrease the gulf between research and practice within arts therapies. Fundamentally, I am a music therapist and the art of music and creativity are vital components in my practice and, therefore, in understanding my work.

Arts-based autoethnographies (Storer, 2014), and arts-based practices in research, have been helpful in developing new thoughts and practices in music therapy (Viega, 2013; Woodward, 2015) and other disciplines. Slattery (2001) makes a case for "artsbased autoethnography" (Ellis, 2004). He contends that an arts-based approach encourages engagement with the unconscious and develops new thoughts (Slattery, 2001). Jung believed that the creation of his psychotherapeutic methods and theories emerged from his sustained "confrontation with the unconscious" (McNiff, 1998, p. 13). Levine (2013) also illuminates the paradox of using artistic improvisation to let go of certainty in pursuit of answering research questions. Improvisation is an engagement in what has not yet been and what cannot be anticipated (Levine, 2013). Arts-based practices may open up our understanding and bring something new into being, which guards against research being a repetition of what the inquirer already knows (Austin \& Forinash, 2005; Levine, 2013). For example, Manovski’s (2014) 
arts-based autoethnography gained further understanding about the culture of marginalisation by analysing singing lessons and engaging in artful narratives, visual images, and music.

Because I framed this research as an arts-based autoethnography, I will briefly define arts-based research (ABR) in MT, and the current project's relationship to it. McNiff's (1998) definition of ABR is specifically targeted to arts therapists as "a method of inquiry which uses elements of the creative arts therapy experience... as ways of understanding the significance of what we do within our practice" (p.13). Austin and Forinash (2005) define arts-based research as a:

"method in which the arts play a primary role in any or all of the steps of the research method. Art forms... are essential to the research process itself and central in formulating the research question, generating data, analysing data, and presenting the research results" (pp.458-459).

In my research, creative practices were involved throughout all stages: the subject under investigation, the data collected (music and creative writing), data analysis (improvisation, creative writing), and representation (photographs, music compositions, creative writing). However, which art practices were used and for what purpose was not predetermined. I was aware of the necessary messiness in qualitative research and need to remain flexible and open to different possibilities. Ledger and Edwards (2011) contend that few MT studies, at the time of their literature review, had fully met Austin and Forinash's (2005) description of ABR despite using the arts at some stage in the process. Ledger and McCaffrey (2015) propose ABR in MT to be a flexible research strategy and resist giving strict definition. In the $3^{\text {rd }}$ edition of Music Therapy Research (2016), Viega and Forinash expand the definition of ABR from the previous edition (Austin \& Forinash, 2005) to highlight the diversity of approaches and current trend of including arts-based practices in any or all of the stages of research. They place more focus on the arts informing and sometimes leading the research process. My research drew on aspects and principles of $A B R$, in accordance with this expanded view of ABR. An arts-based approach fits with autoethnography's "attempt to disrupt notions of normalcy in research" (Slattery, 2001, p. 389). 


\subsection{Music therapy skills supported by art-based autoethnography}

As well as the use of art and creativity in my autoethnographic approach, which has an obvious direct link with practice, reflexivity is a skill both ethnographers and music therapists employ. This potential exchange of skills between practice and research excited me as I wanted my research and practice to support and impact on each other in constructive ways.

Although the two terms are often used interchangeably, in this research I recognise that there are differences between reflective and reflexive practice. Reflective practice is an in-depth review of events to work out what happened, what was thought and felt, and who was involved (Bolton \& Delderfield, 2018). Reflecting on practice may lead to insight about something unnoticed at the time (Bolton \& Delderfield, 2018).

Reflexive practice expands the frame by finding strategies to examine values, assumptions, prejudices, theories, and attitudes. It is about questioning how congruent actions are with espoused values and theories (Bolton \& Delderfield, 2018).

Reflexivity is the skill of being aware of our active role in shaping surroundings and responses to other people and events and the use of "this knowledge to inform our actions, communications and understandings" (Etherington, 2004, p. 19).

The reflexivity required for autoethnography has been thought to enhance and facilitate the use of reflexive practice for occupational therapists (Hoppes et al., 2007), family physicians (Foster, 2013), nurses (Jones, 2012), and vocational psychologists (McIlveen et al., 2010). To help improve this skill, Hoppes, et al (2007) developed a course in autoethnography for occupational therapy students. To be reflexive, we need to be aware of the cultural/social and personal contexts in which we live and work, and to understand the ways in which these impact on how we interpret our surroundings (Etherington, 2004). What autoethnography offers the development of reflexive practice is its focus on linking personal understandings with facets of cultural meaning.

Alongside reflexivity, I became more aware of the similar skillsets ethnography and MT share while doing an ethnography/ethnomusicology paper. Within the autoethnographic approach, I drew on the ethnographic tool of thick description. I found the five characteristics of Geertz's (1973) expanded explanation of “thick description" (p. 3) to be present in my MT reports. I also recognised that in sessions I 
simultaneously participate in and observe what is going on, much like the practice of participant observation in ethnography. Therefore, as an autoethnographer I could build on skills acquired through practice and vice versa.

\subsection{Music therapy literature regarding autoethnography and arts-based research}

Autoethnography and ABR have not previously been widely used and explored in MT (Austin \& Forinash, 2005; Edwards, 2015; McNiff, 1998; Wheeler, 2016), but there is a growing interest in these methodologies, resulting in more exposure and examples over the last few years (Viega, 2016b; Viega \& Forinash, 2016; Woodward, 2015). The first example of an ABR study that is often cited is Austin's 1991 "Grace Street" (Austin et al., 2016). However, other music therapists, such as Carolyn Kenny, have advocated for the use of arts as early as the 1980s (Viega, 2016b). From these beginnings, momentum has grown and $\mathrm{ABR}$ studies have explored a range of topics (Ledger \& McCaffrey, 2015). Recently, a number of MT journals have included special or themed issues on ABR: Music Therapy Perspectives (2016) and the Journal of Music Therapy, winter (2015). Additionally, the online journal of Voices: A World Forum for Music Therapy dedicated a special edition to "the glorious blurred boundaries of art, research, and performance" (Viega, 2017, p. 1).

There is diversity in the epistemology, topics covered, and rationale given for ABR in MT literature, indicating that there is not a fixed ontological basis (Ledger \& McCaffrey, 2015). ABR has been used in MT as a way of more adequately conveying participants' experiences (Rykov, 2011; Viega, 2013): as a vehicle for embodied reflexivity (Gilbertson, 2015; McCaffrey \& Edwards, 2015; Schenstead, 2012); for supporting analysis (McCaffrey \& Edwards, 2015); to elicit unforeseen and unexpected findings (Hoskyns, 2017); to enlarge understanding in both intersubjective and intra-psychological domains (Gerge, Wärja, \& Pedersen, 2017); to enlighten the creative process in MT; and to reveal complexities and integrate aspects of the therapists self (Viega, 2016a, 2016b). MT ABR studies have covered numerous topics, for example a MT cancer support group (Rykov, 2011), developing a method of mentorship in MT (Vaillancourt, 2009, 2011), and examining hip hop songs written by adolescents who had experienced adverse childhood events (Viega, 2013). The 
boundaries of ABR are contested in the literature (Ledger \& Edwards, 2011; Ledger \& McCaffrey, 2015).

Despite the small number of ABR studies in MT (Wheeler, 2016), ABR is increasing. However, there appears to be less autoethnographic examples found and less attention given to this research method. Some music therapists have engaged with autoethnography, but have not yet found a way to publish their narratives (Edwards, 2015). Edwards (2015) encourages students to have an autoethnographic chapter in their thesis due its helpfulness. Music therapy master's theses (Klaette, 2018; Lipski, 2014; Zhang, Shi, \& Hsu, 2016) and doctoral theses (Baines, 2014; Woodward, 2015) have utilised autoethnography. Woodward's (2015) autoethnographic thesis uses artsbased techniques. Her PhD explored using MT in humanitarian aid in Bosnia, Herzegonvia. She noted the aesthetic and reflexive process of this method was helpful to her research.

It was only in 2016 that autoethnography had more of a presence in the third edition of the well-known Music Therapy Research, edited by Wheeler \& Murphy. Stige \& Ledger (2016) note that there is a wide range of ethnographic research emerging, “including experiments with autoethnography” (p.412), citing Woodward's (2015) work, noted above. The chapter on first-person research by McGraw Hunt (2016) briefly discusses autoethnography, again citing Woodward's work, but also includes Bruscia's description of his experiences at the First International Symposium of Qualitative Research (Bruscia, 1996). Other chapters briefly mention autoethnography.

Other published examples were found. Freeman's (2018) autoethnography explored MT with people living with dementia and Devlin's (2018) explored the question, "How do I see you, and what does that mean for us"? when working with nonspeaking clients. However, while it appears that there is a growing awareness of autoethnography, there is a significant lack of published autoethnographic MT research. There are more examples of other first person research/essays that use data from the self (McGraw Hunt, 2016 ), such as personal narratives (Amir, 1996; Rickson, 2004), but these differ in regards to autoethnographic analysis. Furthermore, I note that researchers from related disciplines have informed MT by using autoethnography (K. Lee, 2010; Lewis, 2017; Turner, 2017). 
In conclusion, there is a need to extend and build on the current literature to further determine what ABR and autoethnography may offer MT and their potential detractions. It is an exciting time to use such methods, as they have not yet been institutionalised or constrained by MT discourses, allowing novel exploration of possibilities (McCaffrey \& Edwards, 2015).

\subsection{Detractions, criticisms and their retorts}

I have noted some potential benefits of using an arts-based autoethnographic approach but there is also a need to be aware of criticisms, counter arguments, and ways to manage possible detractions.

\subsubsection{The flaws of the self-indulgent critique}

Autoethnography has been criticised for being self-indulgent due to the focus on personal experience (Atkinson, 2006; Delamont, 2009). However, this critique fails to recognise the inextricable relationship between selves and cultural systems (Ellis \& Adams, 2014). Autoethnography takes the stance that we cannot separate ourselves from how we understand the world, as this plays an important part in how we understand others (Jones, 2012; McIlveen, 2007). Foucault contends that it is ethical to first take care of the self, which includes understanding oneself, before taking care of others (Foucault, 1997b). Therefore, individuals who intensify the relationship of the self are not self-indulgent: they can reinvent themselves as subjects, better fitted to engage with the broader community and act responsibly in relationships (Danaher, Schirato, \& Webb, 2000). On this note, Jones (2012) argues that writing autoethnography can enhance the "therapeutic use of the self" (p. 575) for nurses caring for people with borderline personality disorder. The notion of the therapeutic use of self links with Foucault's idea, in that it refers to evaluating ones morals, characteristics, and expertise in connection with others (Jones, 2012; Kwaitek, McKenize, \& Loads, 2005). Ultimately, this self-examination is used to enhance practice outcomes.

Another reason to refute self-indulgent critique is that there is a need for more personalised research methods, as they can be effective in confronting challenging topics that arise in practice and provide a platform for silenced, marginalised voices. What initially struck me about some autoethnographies was their difficult topics, 
which are traditionally hard to research (see, Averett \& Soper, 2011; Long, 2008; Richards, 2008; Uotinen, 2011). Some examples include exploring sexuality in physical impairments (Neville-Jan, 2004), bodily knowledge and experience in an ICU (Uotinen, 2011), mental illness in the nursing workplace (Kidd \& Finlayson, 2010), fear in a woman's life and the implications for social work (Averett \& Soper, 2011), and the first encounter with death in occupational therapy (Warne \& Hoppes, 2009).

To further understand why it is important that we examine ourselves in relation to social context, I turn to Foucault's concept of governmentality. Governmentality means that individuals are constituted as subjects by governmental practices and normalisation, and that they regulate (govern) themselves to adhere to these (Danaher et al., 2000; Foucault, 2010). The power dynamics that create self-regulating subjects are more powerful when hidden from view ${ }^{12}$. This means that people can be unaware of how they regulate themselves to fulfil governmental and normalising practices. To illustrate governmentality I offer this example, as it demonstrates how our actions/responses can become habitual and subconscious and raises the importance of gaining self-awareness of our own hidden self-governing practices.

She held the kettle and tilted it towards our cups. Our minds poured over who might be interested in reading my proposed research. Music therapists, doctors, allied health professionals, educators, theoreticians were all written down. Feeling satisfied with the list of important people, I started to talk about other matters. After time had elapsed, and my drink had decreased in size and warmth, my supervisor said, "what about patients"? Is this how I unthinkingly and by default placed patients, with the leftover cold dregs in the teacups? Maintaining the prestige hierarchy, where patients were at the bottom.

${ }^{12}$ Foucault's idea about power is that it is not a thing that people have; rather it moves through people and can be seen in our interactions, or when it is put into action (Danaher et al., 2000; Foucault, 2010). 
It could be this low-patient status that makes me continually question whether studying my narratives has anything to offer MT theory. However, we are not just docile bodies: by identifying the regulatory institutions and discourses by which we are produced and how we govern ourselves, we can choose to respond to or resist these normalising practices, and are therefore capable of ethical and moral dimensions (Danaher et al., 2000). By studying the self to identify these practices, we can create new ways of working.

Whilst there are multiple ways to argue the flaws of the self-indulgent critique, it does remind one to have in place measures that will constantly challenge, question, illuminate hidden perspectives/ideas and assist with reflexivity. Accordingly, in the first two years of my study I attended a PhD supervision group that focused on poststructuralism and ethics. Throughout my study, I attended regular clinical and research supervision. Furthermore, a peer reviewer was consulted towards the end of my research regarding the theoretical lens I was developing. The peer-reviewer acted as a critical friend during this stage of the process.

\subsubsection{Critiques based on normalised standards of research}

Other critiques of autoethnography have been based on the inability of the method to meet traditional notions of generalisability, validity, and reliability (Ellis \& Adams, 2014). Ellis and Adams (2014) offer alternative ways to assess these aspects in autoethnographies. For example, generalisability is assessed based on the story's ability to speak to the reader and validity is based on verisimilitude (the reader finds the experience possible, believable, and lifelike). Furthermore, they outline seven criteria to examine autoethnographies: that they

1) "use personal experience; 2) have a familiarity with existing research; 3) describe and/or critique cultural experience; 4) illuminate insider knowledge; 5) break silence and reclaim voice about a topic; 6) manoeuvre through pain, confusion, anger, and/or uncertainty; 7) and be accessible" (Ellis \& Adams, 2014, p. 269).

Aesthetic merit has also been highlighted as an evaluative criterion (Richardson, 2000). Therefore, auteothnography can come under scientific and artistic scrutiny. 
The theoretical approach and Foucauldian tools I present later on address these issues and the seven criteria mentioned above.

\subsubsection{The detraction of the boundless possibilities of art-making}

A possible detraction for using an arts-based approach is that the researcher could become stuck in the art-making process and struggle to produce tangible results (Austin \& Forinash, 2005). McNiff (1998) suggests the greatest challenge of ABR is its boundless possibilities, and Ledger and MacCaffrey (2015) note that choosing art mediums is not straight forward. The university limitations placed on this research project, such as the allocated timeframe and word limit, maintained some boundaries during the art-making process. The research questions also provided boundaries.

\section{Theoretical underpinnings}

This research used a poststructural lens, with attentiveness to social constructionism and feminism. Poststructuralism refers to a range of theoretical positions and links language, social organisation, subjectivity, and power (Drewery \& Monk, 1994; Richardson, 2000). It includes work from Foucault, Derrida, and Butler who have theorised and critiqued language, discourse, knowledge, power, and the subject since structuralism (St. Pierre, 2001). Poststructuralists presume that there is not a real world that can be captured through research and analysis. Rather, "the world and the subject are textual constructions" (Denzin, 2014, p. 36). Meanings are changeable and in motion, contradictory, and inclusive. They do not mirror experience: they create representations of experience (Denzin, 2014).

I argue that social constructionism is underpinned by poststructuralist assumptions (Drewery \& Monk, 1994), such as truth being constructed and multiple. It is relevant because an autoethnographer's ontology would assume "personal reality" to be a psychosocial construction (McIlveen, 2008). Social constructionism fits with this ontology (Drewery \& Monk, 1994). In social constructionism people create meaning within social contexts, using language as a medium. It is through language that we ascribe to ourselves bodily feelings, emotions, and other personal attributes (Rose, 1996). Subjectivity is constructed through language/discourse, as this is where social organisation and power are defined and contested (Richardson, 2000). The discourses available to a person can determine what something means to them (Richardson, 
2000). Due to competing discourses “one's subjectivity is shifting and contradictory, not stable, fixed and rigid" (Richardson, 2000, p. 9). The concepts we use, such as disability or femininity, are socially determined: they don't refer to an independent reality, but instead vary across cultures and time (Barker, Pistrang, \& Elliott, 2002). Therefore, there is no essential meaning or fixed self, which enables the self and meanings to be open to change (Drewery \& Monk, 1994). Accordingly, my research does not look to discover if something is true or not, but instead focuses on how things are constructed and what these constructions do.

What social constructionism means in the context of therapy is that the therapist is supporting the client's self in a continual process of change and becoming, rather than a movement towards an end point (Drewery \& Monk, 1994). In counselling, Drewery and Monk (1994) argue that suggesting that our identities are socially constructed has therapeutic importance. They explain a self-liberating process when essentialist thinking is shed: when a client can locate an issue as not within themselves but as a construction that is congruent with problematic stories, it allows the old problematic self-story to be replaced with a new one.

In MT, Ansdell (2003) describes what happens in a session with a social constructionist orientation as "the musical performance of the self - in - culture" (p.157). That is, clients would be improvising themselves within social/cultural space, instead of finding themselves within a private space that is decontextualised and internalised (Ansdell, 2003). For therapists, considering the dynamics of interpersonal relationships as instances of construction, reminds one to be mindful and vigilant in one's role of addressing power, social control, and politics within one's practice (Drewery \& Monk, 1994; Hadley, 2013a).

A feature of poststructuralism is "concerns about the gendered nature of transmission and interpretation of cultural meaning" (Drewery \& Monk, 1994, p. 304). It is here that I link it with feminism. "Feminism and poststructuralism work similarly to critique and reinscribe normative, hegemonic, and exclusionary ideologies and practices" (St. Pierre, 2001, p. 149). Feminist theorists have illuminated how masculine standards and male domination have influenced traditional research methodologies and epistemologies (Ruud, 2005a). For example, in reading Behar's (1993) article while doing my ethnography course, I came to realise that women have 
been mostly written out of early American anthropology. I recognised the well-cited names of the male authors referred to, but apart from Margaret Mead, I did not know of the less cited women she discussed such as, Ella Deloria, Gladys Reichard, Jean Briggs, and Alice Walker. The women mentioned above were addressing the distancing forms of fieldwork and exploring the possibilities of self-reflexive practice long before it was popular, but were often marginalised for doing so (Behar, 1993). Ideals such as empathy, closeness, intuition, and specificity, have been marginalised by the domination of distance, control, neutrality, and objectivity in a masculine produced social science (Alvesson, 2002; Ruud, 2005b).

The connection of feminism with my research is the idea that patriarchy impacts on everyone, including MT practice and research (Hadley, 2013a). A feminist lens encourages one to "think about, deconstruct, disrupt, and interrogate existing values that operate within the construction of social values" (Edwards, 2006, p. 367; Hadley, 2013a), and therefore in the construction of practice. It emphasises that individual problems are connected to political and social contexts (Hadley, 2013a) and that the personal is political (Hanisch, 1970). Like St Pierre (2001) explains, to do this research I needed to do the feminist work of paying attention to my gendered experiences.

A further connection with feminism is that feminist theory has much to offer disability studies, and vice versa. Disability's inclusion in feminist examinations is important, given that disabled scholars have noted the exclusion of disabled bodies in feminist theory and practices (Wendell, 2001). The pervasive feminist assumption of autonomy and independence, feuling female empowerment, undermines some disabled women's struggles (Garland-Thomson, 1997). However, contemporary feminist theory has become self-critical and diffuse, speaking in "feminisms" and of "conflicts in feminism" (Garland-Thomson, 1997, p. 21). Adding to the conversation between disability studies and feminism would be beneficial and embraced within this contemporary space.

Feminist writers often insist on the embodiment of the subject. This is because the illusion of the universal disembodied person/researcher is that of the male, consequently denying the female sex (Rose, 1996). Embodied research advocates for 
a reinsertion of the body into our thinking in subjectivity. Knowing through one's body, in my case a female body, is integral in the subject matter of my research.

\section{Data Sources}

I am the only participant in this research. There are no other direct participants. This is because my investigation centres around how I understand and implement my work, as well as how cultural forces have impacted on my practice as a therapist, rather than how clients respond to and engage in sessions. That is, I am examining myself, society, and culture. To answer my research questions, I am using myself as a historical and cultural artefact (Rose, 1996).

I draw on three groups of data during my analysis: 1) data relating to my MT practice; 2) data relating to my own personal experiences; and 3) external sources of data that relate to cultural/social discourses and practices.

\subsection{Music therapy data}

I accessed data through "clinical data-mining", "the metaphorical mine of information contained in therapists' routinely kept records" (Epstein, 2002; O'Callaghan, 2009, p. 16). Clinical data-mining included documentation of individual and group sessions from three contracted facilities, and clients from private practice. The musical material used, and songs composed during sessions were also "mined". I had kept a reflexive journal regarding thoughts about my practice since 2011, which was a significant data source as it detailed my experiences and changing thoughts. Notes that I had taken during clinical supervision were also included.

\subsection{Personal experience data}

Personal artefacts that provided data from my life included: My personal journal, my medical records, my own music compositions/music making, emails, and photographs.

\subsection{External cultural data}

Literature was treated as a source of data. This helped to locate myself and the positions I took within relevant discourses in order to better explore the subjectivities available to me. Sources of literature included writings about MT, other allied health 
professions, chronic illness and disability. Research literature, as well as creative/poetic writings, were utilised. Public blogs relating to disability and illness provided data regarding other people's experiences to use in dialogue with my own. Various professional documents, such as codes of ethics, practice standards, school contracts, renewing practicing certificate guidelines, ministry of education documents, and Kia Orite, the national code of practice for tertiary education students with impairments in New Zealand, were also drawn on as sources of data.

\section{Data analysis}

For the data analysis, I chose to use tools from Foucault's critical ontology of ourselves. This is an historical analysis and investigation consisting of an archaeology and geneology. The archaeology is the overarching approach focusing on the formation of discursive rules and how artefacts fit together in a historical moment. The genealogy, which is based on the archaeology, is a closer analysis of how discursive rules produce us as subjects. The genealogical analysis has three open questions and domains which align with my research questions and are as follows:

- "How are we constituted as subjects of our own knowledge" (Foucault, 1984b, p. 49)? This is the domain of truth (Foucault, 1984a). It relates most strongly to my first and third research questions.

- "How are we constituted as subjects who exercise or submit to power relations" (Foucault, 1984b, p. 49)? This is the domain of power (Foucault, 1984a). It relates primarily to my first three research questions.

- "How are we constituted as moral subjects of our own actions" (Foucault, 1984b, p. 49)? This is the domain of ethics and becoming moral agents (Foucault, 1984a). This relates to all of my research questions.

These three axes of analysis-knowledge, social relations, and selves-are not contradictory but complementary. They assist in turning oneself into a subject to uncover constitution and practices of the self (Kay, 1999). Foucault comments that, "it seems to me, that all the so called literature of the self-private dairies, narratives of the self, etc.,--cannot be understood unless it is put into the general and very rich framework of these practices of the self" (Foucault, 1984a, p. 369). He contends that practices of the self are historically analysable; but without targeted tools they can be 
challenging to analyse because techniques of the self are often invisible and frequently linked to supporting others to manage themselves (such as the role of a therapist) (Foucault, 1984a).

There are key features to this historico-critical inquiry of oneself that are of interest here. Foucauldian analyses of the modern subject uncover the ways that marginalised individuals, like the disabled, have been excluded and regulated, which is of interest to this study. The critical ontology has to be considered as an ethos, an attitude, a philosophical way of life where we both critique what we are and experiment with going beyond the limits imposed on us: a helpful ethos for a reflexive practitioner. It is also an adaptable and flexible approach that needs to be translated and developed for each particular inquiry for the analysis of specific problems in specific contexts (Foucault, 1984b; Yates \& Hiles, 2010).

With this in mind, the manner in which I have gone about this critical ontology may be different to others' interpretations of Foucault. Foucault believed that it is a tribute to another's thought to "precisely use it, to deform it, to make it groan and protest" (Foucault, 1980, pp. 53-54; St. Pierre, 2001). Furthermore, Foucault developed no specific steps for this analysis (Hook, 2005). What he does offer is a mode of critique, an array of defamiliarising and reconceptualisation tools (Hook, 2005). Therefore, I went about this analysis in the following way:

1. Firstly, I identified personal experiences and examples from practice (historical events) that were unsettling or remained alive for me, and were therefore relevant to the research questions.

2. Secondly, as Foucault proposed the critical ontology to be archaeological in its method (Foucault, 1984b), I illuminated the discourses that articulated what was said, done and thought within the historical events i.e. the above experience. I looked at the conditions under which the experiences had taken shape, defining the discursive space and illuminating its rules of formation (Kay, 1999; Rose, 1996). That is, I looked for what was accepted as "true" within certain discourses/conditions. Truth is the first domain in the critical ontology of the self.

3. Thirdly, as Foucault proposed the critical ontology to be genealogical in its design (1984b), I critiqued and analysed the data and discursive "truths" from 
the events selected in a way that addressed the three domains (truth, power, ethics) and questions. This was not a history of a person or a discipline but an investigation of the practices and techniques that constituted them (Rose, 1996). The genealogy looked for specific meanings not universal ones (Hook, 2005). I used the following Foucauldian tools to do the genealogical analysis:

3.1 I found moments of problematisations and problematised my data. Problematisations develop when we reflect, discuss and become concerned with how we live in the world (Packer, 2011). They come about in everyday practices where conduct has become problematic to others and ourselves (Rose, 1996). Problematisation refers to exploring the conditions in which problems were possible, instead of evaluating them. By doing so, "it unsettles our tendency to accept a problem as 'given' and allows us to see it instead as arising from specific cultural and historical configuration" (Packer, 2011, p. 376). It was a tool that helped me to unsettle the "truths" in my practice.

3.2 I looked for moments of governmentality in my data. Foucault's concept of governmentality means that individuals are constituted as subjects by governmental practices and normalisation, and that they regulate (govern) themselves to adhere to these (Danaher et al., 2000). For example, people govern themselves to stick to the road rules. So, I looked for ways that I responded to discursive practices and cultural forces by which I was produced. By doing so, how I governed myself became more visible and opened up such practices for interrogation and transformation, enabling choice in how to respond to or resist these normalising practices which may enhance capabilities of ethical and moral dimensions (Danaher et al., 2000). MT practice is part of a therapeutic tradition that has played a role in the ways human beings have regulated others and have regulated themselves within certain games of truth (Rose, 1996). Therefore, governmentality was a relevant and important aspect of the analysis. Finding moments of governmentality illuminated the domain of power, whilst opening up possibilities for ethical change.

3.3 I identified moments of "the ethical care of the self". This related to the third domain (ethics). I looked specifically for examples or moments of 
moral conduct, the ways I was invited to recognise moral obligations, and therefore changed in order to become an ethical subject (Packer, 2011). In contrast to the normalised conception of the self, idealised through experts such as teachers, doctors, therapists etc., Foucault's ethic was disconnected from "the sciences of the self and their associated professional experts" (Randall \& Munro, 2010, p. 1487). This ethic of the self can be understood in terms of the ways in which one tries to shape and master themselves and their life (Randall \& Munro, 2010). From the care of the self the subject is constructed through practice rather than contemplation (St. Pierre, 2001). I looked for clinical and personal practices that changed, such as the way I wrote my clinical notes. I attempted to fulfil the necessary attitude: Foucault indicates that this confronted what I was thinking and saying with what I was doing (St. Pierre, 2001). The ethical care of the self is a self-forming activity with the goal to both "produce oneself as the ethical subject of one's actions as well as to create one's life as a work of art” (St. Pierre, 2001, p. 156). I was asked during the confirmation event for my $\mathrm{PhD}$ proposal if there was space for agency in my Foucauldian approach to the analysis. The process of the constant creation of the self, and the use of art, is how I used agency within this research. Whilst his earlier work is critiqued for its lack of agency, in his later work Foucault accepted and developed this creative and self-care form of agency. This leads into the final stage in my analysis.

4. Fourthly, I created myself as a work of art. Relating to the domain of ethics, from the data gathered and analysed in chapters $4,5,6$, and 7, I then created myself as a work of art in chapters 8 and 9. Foucault states that "from the idea that the self is not given to us, I think there is only one practical consequence: we have to create ourselves as a work of art" (Foucault, 1984a, p. 351). This fits with his idea that the critical ontology of ourselves should experiment with the possibility of going beyond the limits imposed on us and create something new. I took up this notion literally and used arts-based practices during the research process to help open up new practices and understandings. The use of art during research analysis can provide a way to gain insights not knowable 
by other means and opportunities that further examine the data (Austin \& Forinash, 2005; Ledger \& Edwards, 2011). As Foucault so eloquently put it, "why should a painter work if he is not transformed by his own painting" (Foucault, 1997a, p. 131)? It was during this stage of the analysis where I consulted with a peer reviewer. This involved verbally presenting some of my findings from the analysis and discussing these with her.

\section{Ethical considerations}

This research project received ethical approval from the human ethics committee at Victoria University of Wellington (approval: 22214) in November 2015. Although I am the only participant in this research, other people could also be implicated in the use of my experiences. Relational ethics and concerns were an important dimension of the study that I needed to keep in mind throughout the writing and research process (Ellis et al., 2011). Relational concerns were important because I had continued relationships during and post study with the people who may be implicated, for example my husband (Ellis et al., 2011). Although I do not use the full name that I usually go by in this thesis ${ }^{13}$, I am still identifiable as the researcher. Therefore, family and supervisors could not be granted anonymity, even with the use of pseudonyms. Consent was sought from those who were implicated in this way (appendix 1). It was clearly outlined during the consent process that they may be identifiable. Sharing what I wrote, and allowing them to respond to it, was implemented in this situation.

In respect to other people (teachers, doctors, nurses etc.) and people I have worked with, I used composite cases/stories: emotionally rendered representations of experiences using fictional characters and settings. These focused on common unresolved problematisations that I faced in my practice with multiple students. By keeping the composite cases attentive to problematisations, the issues themselves were not fictional, but rather the examples used to explain them were fictionalised for the protection of clients and staff. The use of this technique did not require consent from the client/family and was used to ensure client confidentiality. However,

13 The reason for this is discussed in the conclusion. 
informed consent was sought from the facilities I worked at to generate data for the composite cases through "clinical data mining" (Epstein, 2002). The advantage to using "clinical data-mining" (Epstein, 2002), was that the research could be conducted in a non-intrusive way to my clients (compared with data generating research methods) as it involved information that had been obtained during routinely kept records (O’Callaghan, 2009).

Although in most instances I used composite cases/stories, particular examples of my work with three clients were used to illuminate the findings. When a composite story was not used, I sought consent from the clients and their families to be included in this research (appendix 1). To help uphold their confidentiality, identifying characteristics, for example, gender, name, place etc. were altered. The importance was the crux of the story as opposed to the precise detail (Ellis et al., 2011).

This research conformed to the University's Treaty of Waitangi Statute. Participation and protection were supported by consulting with a Māori advisor at Victoria University of Wellington. We had four meetings in which we discussed Māori models of health and related these to personal experiences and practice. This was important because I have worked with Māori students in my practice. MT has similarities with Māori philosophies towards health as it is based on a wellness or holistic health model and has synergies with the concept of Te Whare Tapa Whā, outlined by Mason Durie $(1985) \cdot{ }^{14}$

The ethical considerations of doing this autoethnography did not just involve protecting those implicated by my stories, but protecting myself as well (ChathamCarpenter, 2010). The topic under investigation was a challenging one. As indicated in the literature, PPs can experience discrimination, marginalisation and microagressions. By undertaking this research, my illness/disability was disclosed to people who previously did not know of my situation. Autoethnographic research can make the author vulnerable as they expose their thoughts, experiences and struggles (Fahr, 2006). Some self-protection strategies include waiting until being in a good space to write, choosing and experimenting with what to tell and what to omit, understanding that what is written is permanent, and anticipating future vulnerabilities

\footnotetext{
${ }^{14}$ Further discussed in chapter 7
} 
(Chatham-Carpenter, 2010). I used all of these strategies throughout the research process.

Confidentiality, consent, and self-exposure were troubling issues at times. However, I am satisfied with my decision to use a mixture of composite stories and sought consent. Furthermore, using a poststructural lens meant that I focused on texts/discourses rather than personalities and characteristics. This mitigated some of the potential danger in making myself too vulnerable, as it relocated the personal into the domains of culture, language and the subjectivities available to someone in my position.

\section{Prelude to, and structure of, the following analysis chapters}

Now that I have outlined the methodology, I hesitantly strike a match; flame to candle; I first have to show you what's there. When it comes to invisible illness/disability, we are "finding our way in the dark" (D. Wall, 2005, p. 4). Darkness breeds fear. Where there is fear stigma can grow and intensify (Coleman-Brown, 2013 ) and like wind to a candle it can blow it out or make it flicker. Can I illuminate that which is unseen in these conditions? Italics are how I mull over problematisations - head tilted, squinting a little more. Hershey (2011) cautions writers with disabilities: "Your happiness will be called bravery, denial. Your sadness will justify their pity. Your fear will magnify their fears" (p.134). It makes lighting this candle difficult for I do not want those things; there are many hands that pull at my arms (even my own). A part of me wants to "bury what it is I do not want to see, (and) forget that this also creates my world" (1+alifax, 2009, p. 20). Sometimes that part wins. In handwriting you can see how I govern myself. In the following analysis, I'm searching for ghosts not running away from them. This last change in font represents the ethical care of the self: An attempt to change from the mess. In short, the following analysis chapters have, paradoxically, been very challenging, illuminating and exciting to write.

Four analysis chapters follow (chapters 4, 5, 6, and 7). Although they have been written as separate chapters, the "truths" discussed across the chapters work together to create the ways in which disability and illness had been predominantly understood 
in my practice, and therefore how practice was implemented. The chapters relate to and address different research questions at different times. I will draw the findings of the research questions together in the discussion chapter following the "creating the self as a work of art" chapters.

Table 1: Fonts used for personal experiences during analysis chapters

\begin{tabular}{|l|l|}
\hline Italics & $\begin{array}{l}\text { Problematisations: events/experiences that problematise the } \\
\text { truths of the particular discourses discussed (truth). }\end{array}$ \\
\hline Bradley Hand & $\begin{array}{l}\text { Governmentality: events/experiences where I have } \\
\text { governed myself according to the discursive truth } \\
\text { (power). }\end{array}$ \\
\hline $\begin{array}{l}\text { Prestige } \\
\text { Elite Stud }\end{array}$ & $\begin{array}{l}\text { Ethical issues: ethical care of self } \\
\text { (ethics). }\end{array}$ \\
\hline Times New Roman & Normal text. \\
\hline
\end{tabular}




\section{Chapter 4}

\section{Seeing the Unseen: The Shadow Puppets of Disability and Illness}

"Shadow puppets": photograph of the shadows of the figurines others and I made at the Te Papa exhibition of "curious creatures and marvellous monsters".

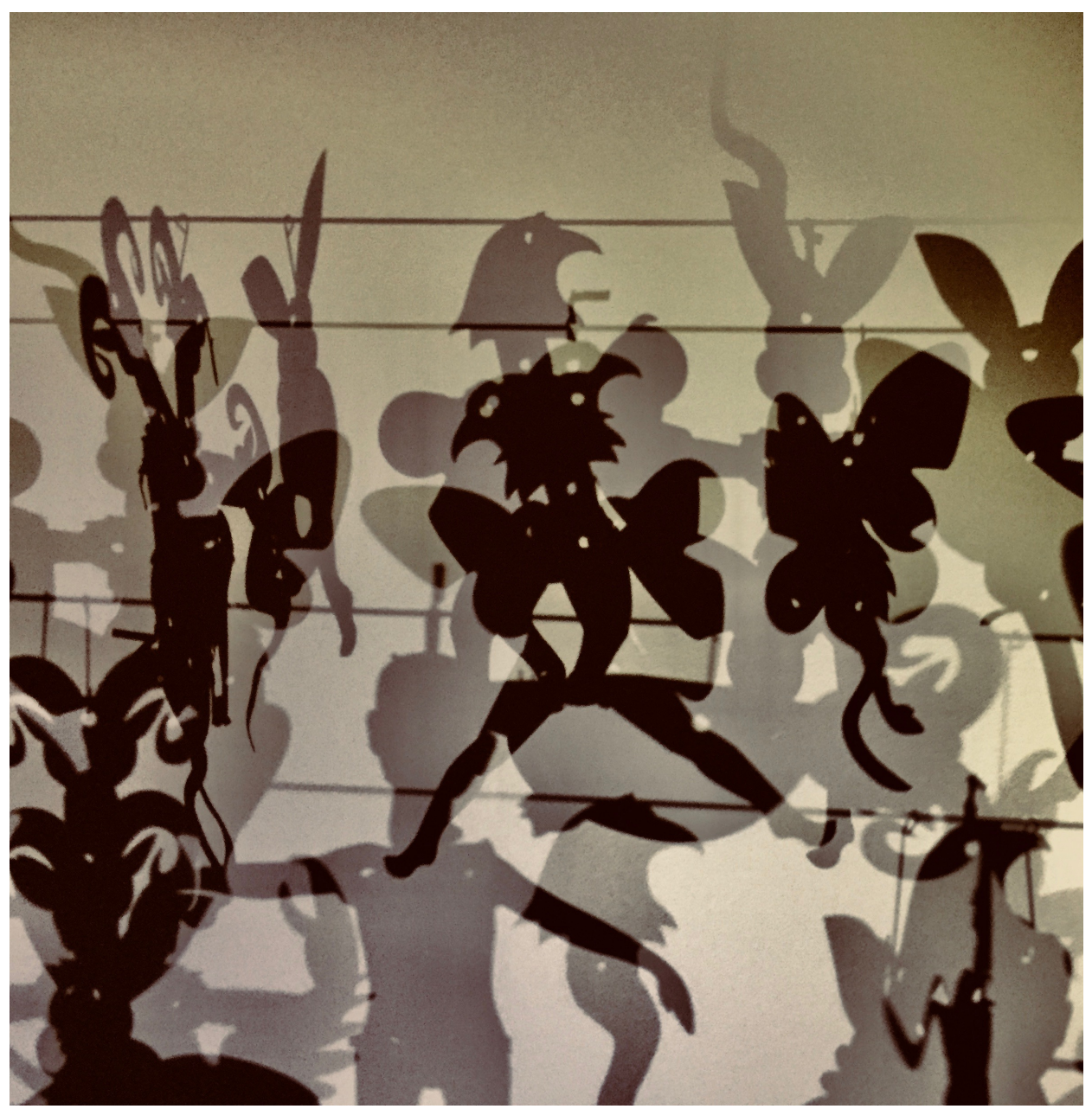

[Shadows of strange figure shapes. Some bird or animal like and others human like] 


\section{Introduction}

In keeping with autoethnography's commitment to show not just tell, I introduce this chapter with an exercise of "what do you see"? This frames the analysis that follows while describing the experiences that lead me to problematise the discursive practices of illness/disability identification in my practice.

\section{What do you see? A personal account}

I light the first candle.

What do you see?

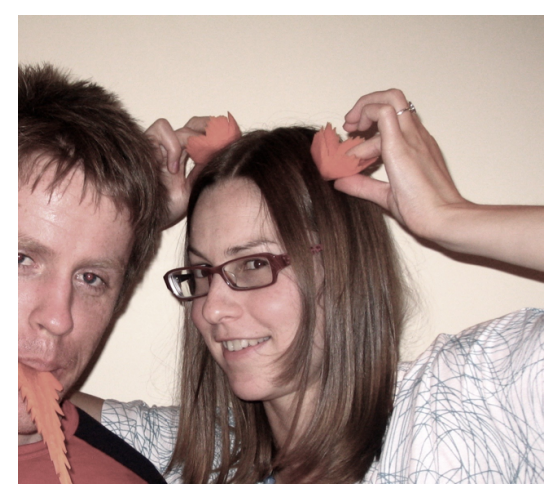

[Photograph of my husband and me. I am holding two carrots, that are sculpted into an ear like shape, up to my brown hair. My husband has a carrot sticking out of his mouth].

I light the second candle.

What do you see?

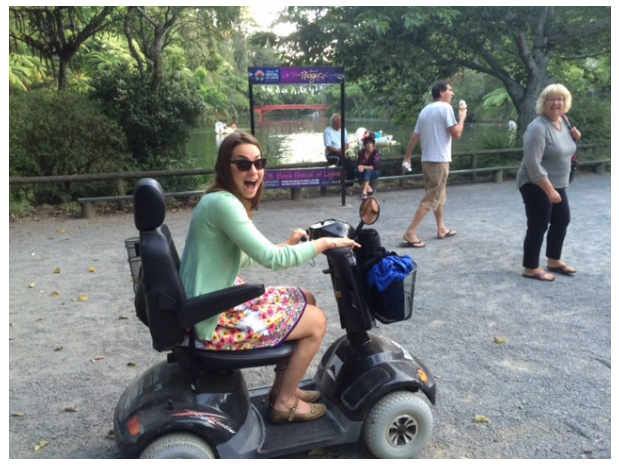

[Photo of me on a mobility scooter with an exaggerated smile] 
I light the third candle.

What do you see?

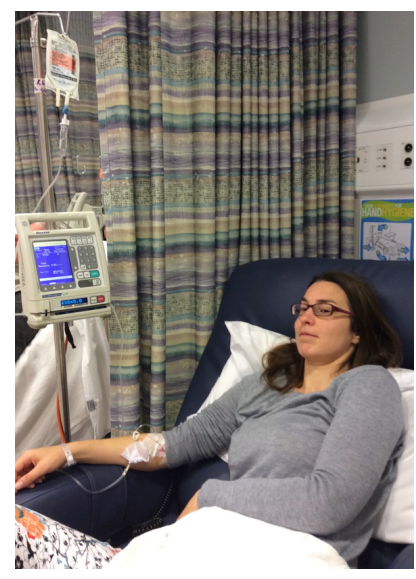

[Photo of me in a hospital chair getting intravenous medication]

Which picture did you think I was struggling with my health?

Which picture did you associate with disability?

Which picture did you not associate with either disability or illness?

My questions are flawed, as to answer them one has to rely on the discursive practices of illness/disability identification which create the continued fallacy that health and non-disability and illness and disability are categories that are obvious, knowable (Samuels, 2014b) and easily identifiable. It was in the first photo that I was most unwell and disabled. Here, I am at my brothers $30^{\text {th }}$ playing with the carrot decorations. I may have looked like a happy lady or a jokester bear impersonator. A magic trick to the eye as this is the body I felt:

A face that earlier cried in protest when mum said, "I'm taking you home, you can't even walk". A chest where each breath was a labour of love. With pulse pressure like a collapsible chair folding down to barely there, there was nothing for my body to rest on. It worked harder, faster, pumping at a rate that rings cardiac alarms. I felt a brain struggling to connect words to tongue. A connection lost because the electricity was switched off for not paying with enough blood. I felt a stomach that 
tried to eat a few small bites, only to be bitten back. I starred at the plate of food hoping that hungry eyes could digest food and bypass the stomach. I felt flesh that stabbed and ached for a position that could release a thumbscrew. I felt a mouth that smiled and laughed. A person that was there, while not quite being there: There is everywhere with dizziness. The self not quite occupying the body, lost

Somewhere

between the space of consciousness

and

nothing.

Scared, at a ropes end while enjoying the life jacket of laughter from family.

Leaving early, because participation got too hard. Leaving early, because one can't lie down in the middle of a restaurant.

Admitted to hospital the next day, where here you can observe that I am not well from the surroundings. However, I felt more comfortable here, than I did at the restaurant.

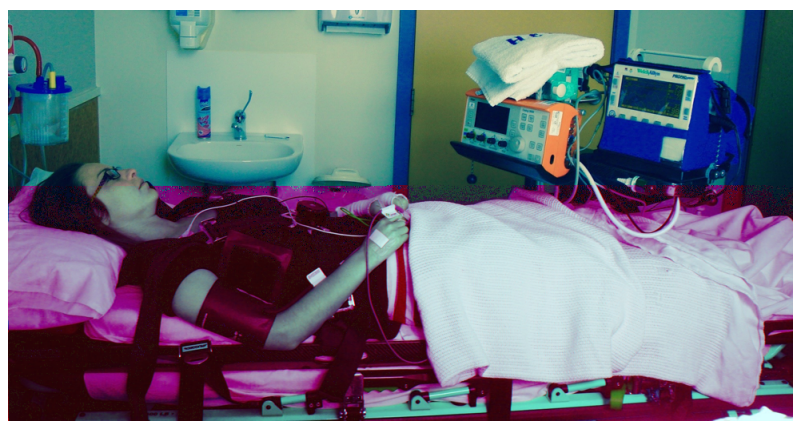

[Photo of me lying down in hospital with blood pressure and heart rate monitors]

Illness can look like nothing.

Disability can look like nothing.

Something comes from something.

Nothing comes from nothing.

The signs by which I knew my old self, and signs that resonated with my new self, were missing: The self erased (Hogue, 2011). Who was 1? (No)body (Erevelles, 2011)? I was confused, "fireworks look like jelly fish, but jelly 
fish don't resemble fireworks" (Brinks, 2011, p. 363), the non-disabled can look like the disabled and the disabled don't resemble the disabled.

The second photo is of me at the festival of the lights. I may look like a woman in her 30's with a disability? Or perhaps a woman who seemed to have stolen a mobility scooter for a joy ride? Neither are true because in that moment, I didn't feel disabled in my body. I didn't feel disabled by attitudes (the staff gave me the scooter no questions asked). I didn't feel disabled by access: for, like those riding legs, I was seeing all I wanted to see. But as I sat on this symbol of disability, others identified me as disabled. Sometimes I am disabled when I am in my wheelchair, especially when access issues present or my body fatigues, but on this occasion, I wasn't experiencing disability.

The third photo is of me receiving routine monthly treatment. It may look like my health is compromised? However, my health had been stable due to the immunomodulatory therapy. At this stage I was thinking of illness as coexisting with health. Health only becomes meaningful when it is confronted with illness (Lindsey, 1996). A high level of health does not mitigate the level of illness. Therefore, as I considered myself to be healthy here, I was thinking of this medicine more as my mobility device, or as my assistive technology, for the treatment won't cure. This fits more within a disability framework than thinking of myself as i11. As such, this picture is of me using assistive technology for my immunodisability.

The point of "what do you see" is to demonstrate how the ways we identify and position others, in terms of disability and illness, may not be how they identify or position themselves. Furthermore, their identity can constantly change between disability/non-disability and healthy/unhealthy. This counteracts the dominant 
discourses around illness/disability identification that are based on the continued fallacy that disability is a category that is obvious, knowable, and unchangeable (Samuels, 2014b).

As my work has mostly been in educational settings, I have focused on this area in the following analysis. The predominant way students with disabilities are positioned by others in education often relies on three sub-discourses of illness/disability identification: observability, expertise, and normality. These three discursive practices of identification have influenced my practice at times, which can be problematic: "fantasies of identification" can create an experience of bodily/textual dissonance (Samuels, 2014a, p. 2), as noted in the writing above. Ultimately these discourses work together to overwrite bodily knowledge and experience, which have implications for how others are positioned in therapy.

To structure the chapter, the construction and effects of the "truth" -that disability and illness are easily identifiable-are discussed by looking at the three sub-discourses that form the headings under this truth. Within each sub-discourse, I will firstly discuss the discursive formation in my music therapy (MT) practice and broader social context (truth). Examples are given that uphold this "truth" in my practice, the contexts I work, the profession, and literature, as well as in my experiences as a person with a illness/disability. Secondly, I discuss the power effects of the discourse (power). Power effects refer to the potential impacts of submitting to or exercising power relations within certain games of truth. To end the chapter, some preliminary ethical considerations will be discussed and a more detailed exploration of ethics will occur in chapters 8 and 9. I note that not all MT practices/orientations support this "truth". This analysis is an example of its particular construction and effects in my earlier practice and contexts where I live and work. 


\section{2. "Truth": Disability and illness are easily identifiable}

Three interrelated sub-discourses that help to sustain the "truth" that disability and illness are easily identifiable are discussed:

- Observability (visibility/audibility)

- Expertise

- Normality

\subsection{Observability (visibility/audibility)}

\subsubsection{Discursive formation}

Visibility and audibility have played a role in disability identification. Like many features of western modernity, the fantasy of identification is predicated on an epistemology of visibility, in which identity can be easily read upon the body (Samuels, 2014a, p. 17). The impulse to privilege the visible often arises out of the need to reclaim signifiers of difference which dominant ideologies have used to negatively define minority identities (Samuels, 2013). What disability looks like has been influenced by the practice of charity, such as poster children that often emphasised visible characteristics (Longmore, 2013). While some affirm western culture's predominance of vision and ocularcentrism (Siisiainen, 2008), there is the subjugated narrative of the role the ear plays in identifying illness/disability, which is important to MT. Disability identifications have largely been influenced by western medicine, which scanned bodies in search of identifying characteristics and prioritised sensory perception and empirical observation as the basis of its knowledge (Foucault, 2003; Siisiainen, 2008). Auditory-pathological signs of the body have also had a role in grasping the "truth" and taking charge of the living body (Siisiainen, 2008). Modern apparatuses of power needed ears and eyes (Foucault, 2003; Siisiainen, 2008). I found that some MT practices also draw on both ears (music/audibility) and eyes (visible observations/visibility) to identify levels of illness/disability, which together created the discourse of observability in my prior practice.

The need for observability in Aotearoa, New Zealand (NZ) practice arises from the requirement of both the Ministry of Education and Ministry of Health for evidence of therapy's effectiveness. Evidence-based practice often rests on the therapist using 
observable disability/health identification to secure and continue their work, as observable evidence can determine priority and allocation of funding (Edwards, 2005b). The hierarchy of evidence positions opinions, such as the client's, at the bottom, and objective observable indicators, which are used in randomised control studies and meta-analysis data, at the top (Aigen, 2015).

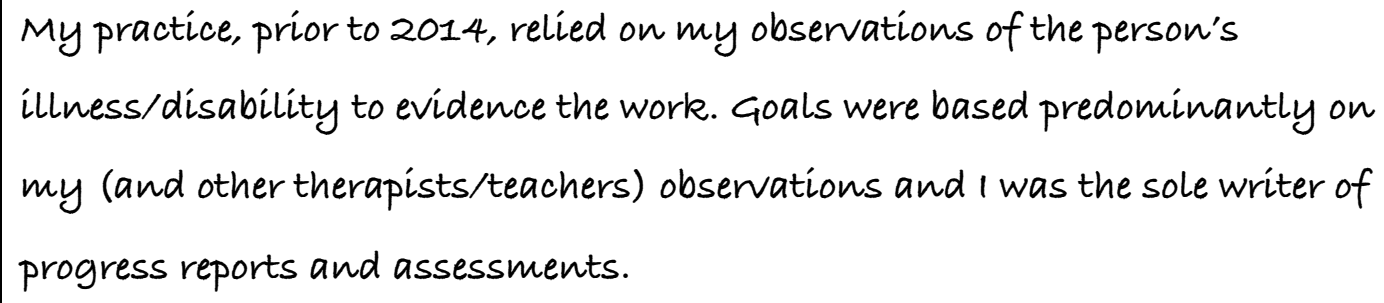

Within my workplace in Aotearoa, the belief that the level of disability is observable influenced processes and discussions, with labels designated to students such as, "low functioning" or "very high needs". These diagnostic terms and "medicoadministrative" (Foucault, 1980, p. 176) truth-making are used as part of the Ongoing Resourcing Scheme (ORS) for regulating school funding allocations for disabled children. This scheme is "predicated upon the belief that disability can in fact be measured, named, and quantified" (Samuels, 2014b, p. 123).

In this context, it is not surprising that in traditional MT it has been common to report on the most observable aspects, which are not always the most relevant or important (Rickson \& McFerran, 2014, p. 137). Kenny (2006) states that "we speak only of observable data... we rarely mention that music goes beyond sign to spirit" (p.10). Relying on observation is common practice in supported learning in NZ. In reviewing articles in the New Zealand Journal of Music Therapy from 2010-2018, regarding work with children in educational settings, examples that focus on observable measures and frameworks based on disability obviousness were found. Churchill \& McFerran (2014) outlined the need for a specific assessment protocol for someone with "severe-profound multiple disabilities" (p. 8). The literature reviewed by Churchill \& McFerran (2014) suggests that therapists can identify people with profound disabilities based on visual criteria such as "skeletal deformations" (p.9). Other identifications mentioned could apply to the human experience of many, such 
as "little control...over their own body" (p.9) and "difficulties expressing themselves clearly and consistently" (p.10). In Churchill \& McFerran's (2014) review of the assessment literature in MT, no assessment tool included culture/spirituality, most likely because these aspects of health/disability are less observable. Interestingly, my own assessment sheet between 2009-2010 relied on observable measures much like tools such as the Beech Brook Group Therapy Assessment Tool (Layman, Hussey, \& Reed, 2013).

In the review of other articles, visual observations were given primacy to acquiring knowledge about a person and used to obtain proof and demonstration of music therapies effectiveness. For example, many observable goals were utilised such as, increased use of left hand (Rickson \& Twyford, 2011), eye contact (Warren \& Nugent, 2010), and "uses simple motor actions" (Ayson, 2011, p. 17). Aural observations included, "speech clarity" (Rickson \& Twyford, 2011, p. 70). In Twyford's (2012) article, peers of students with disabilities also drew on obvious signs that they measured against "normal" standards. Some of their comments included, "I feel he has a new confidence and seems to walk more upright" (p.58), "he knew how to say one, two, three, go!" (p.55).

Music itself is not innocent when it comes to illness/disability identification. Music making has been used to interpret a pathological message from the body (Siisiainen, 2008) and can be understood to represent illness/disability (Straus, 2006, 2014). The Music-Based Scale for Autism Diagnosis developed by Bergmann, Sappok, Diefenbacher, \& Dziobek (2016) is one example. Aigen (2014) suggests that music can become pathologised in psychodynamic practice. For example, arrhythmic playing can be thought to represent a disordered state and progress considered when the client can keep rhythm with the therapist. Like Rickson (2006), I have previously noted beating patterns as an indication of impulsivity levels in practice. Therapists using the Iso principle, endorsed by Altshuler in 1948 (W. Davis, 2003) match the patient's own level of rhythm, melody, timbre as it is assumed that if the patient feels that the therapist is ready to "entrain" with them then this encourages the patient to then "entrain with the therapist's rhythms, which ideally will reflect healthy patterns" (Kenny, 2006, p. 90). Therefore, the more the client sounds like the therapist, the healthier they are believed to be. Additionally, in an analytically-orientated therapy process, the primary focus seems predetermined as a symbolic display of the 
unconscious (De Backer \& Van Camp, 2003; Rolvsjord, 2010). Music has been used to interpret messages from the body/mind within MT assessments such as the Individual Music-Centred Assessment profile for Neurodevelopmental Disorders (IMCAP-ND) by Carpente (2013).

\subsubsection{Power}

The discourse of observability produces the following power effects, which are listed and then further discussed below:

- Accommodations for people with "invisible" disabilities are a struggle to obtain (misunderstood/judged/dismissed).

- Caseload priority and access to therapy can be influenced by "identifiable" measures

- Therapeutic assessment and areas of focus in sessions can be directed by what is observable-operating on a superficial level.

- Music becomes an instrument of disability knowledge, control and normative behaviour.

When your impairment is invisible, you have to constantly remind people of your needs (Wendell, 2001), and push to secure the assistance and resources you require (A. Davis, 2005). The minimisation of invisible illnesses/disabilities is prevalent (M. Williams, 2003), due to both the idea that the only disability worth taking seriously is visible (Stone, 2005) and the connection between visibility and empathy (Honeyman, 2014). This can lead to accommodations not being sought.

Common phrases, such as, "you look well”, are the site of a daily struggle for accommodation. In the case of disabled parking, stereotypes of physical disability being both obvious and permanent shape the social surveillance and policing around disabled parking spaces (Samuels, 2014b).

The wheelchair sign at the parking lot mocks my walking legs. I bow my head and avoid eye contact as I leave my car hoping no one will confront me for parking in the disabled spot. Dominant cultures' insistence on visible signs to legitimate impairments (Samuels, 2013) makes for an ongoing burden of proof (A. 
Davis, 2005; Honeyman, 2014). A stranger's cross-examination of my disability leaves me feeling distressed. This type of surveillance is common: "Every nonvisible disabled person has a host of stories of being challenged by strangers in parking lots" (Samuels, 2014b, p. 132). I need a way to turn myself inside out, for explaining myself is tiring. I miss not having to. It's another layer of fatigue.

While this example is about parking, the effect of this truth on a therapist with an invisible disability is that they may not seek accommodations, or may struggle to obtain accommodations, in the workplace.

Disability/health identifications used by practitioners influence caseload priority and access to therapy. Ultimately deciding which bodies matter, and which are yet to become critical matters of concern (Butler, 1993; Erevelles, 2011). However, observable measures are often not representative of the disability experienced and create a false hierarchy. Ros, who has autism spectrum disorder, points out that her greatest ability of expressing herself verbally is also her greatest disability. Because she is articulate and intelligent, others assume she is confident and comfortable on the inside too (Prizant, 2015). The implications are that someone identified as "high needs" may be prioritized for services over someone with "low needs", even if the experience of disability is insignificant in the former and significant in the latter. Members who don't bear the signifier of difference or look non-disabled are rendered invisible and marginalised within an already marginalised community (Samuels, 2013; L. Walker, 2001). Visible abnormality is at the top of the hierarchy while those without clear markers are minimised, or met with distrust (D. Wall, 2005): "Yet there is no reason to believe that the invisibility of a disability itself necessarily lessons its impact or makes disability less serious" (A. Davis, 2005, p. 154). Understanding only tangible impairments as disability impacts on funding priorities, societal attitudes, and research initiatives that don't direct or guide efforts meaningfully for those with nonapparent impairments, creating marginalisation (Feldman \& Tegart, 2003; Humphrey, 2000; Wendell, 2001).

A colleague referred a teenage boy who did not receive ORS funding. He was articulate and hid his learning disability to blend in with the class. The referral was made due to self-harming behaviours. I tried all avenues to get his sessions funded- 
looking in unlikely places. But the reality was, there was no public or trust source for his sessions to be funded. His internal struggle was made less a priority than the other students I see who were determined as 'disabled enough for funding'.

Alternative understandings of disability, not based on observable and negative ontologies, are often excluded from educational discourses around funding if they do not fit the prescribed fictions (Campbell, 2006).

Therapeutic assessment and areas of focus can be directed by what is observable despite not always being the most relevant or helpful, as demonstrated in the two accounts below.

At the specialists, I told him that my hands and feet were burning. I couldn't sleep. It's like I'd spilled boiling water on them. He said like all my symptoms it would wax and wane. It was like when he renovated his living room he said, "It was hard at the time, but now I don't think about it". Being diagnosed with an illness that significantly impacts daily life was not met with support around how to manage day to day (Reynolds \& Prior, 2003). Like fatigue, pain is often an unmet need: "pain needs a witness, but witness often requires visual cues" (Honeyman, 2014, p. 299). I told him that I wanted to paint my extremities in flames to communicate that it would be more akin to a living room burning down so his assessment could offer more of the support I needed.

At work, active participation is pleasing to the eye (invisible symptoms don't meet the eye, they can't please it). Yesterday Oscar sat slumped in his chair, spending most of his time resting, sleeping, and recovering. Today he has music. With the rhythmic drive his body comes alive, moving, creating, vigorous in its efforts. What a success they all cry: flashes of cameras fill the space. I join them in smiling and keep pushing for such pleasing responses where his disability looks to have reduced. He was on fire. But come Saturday I will not see him. It is 
not my day for visiting. And I wonder, should I have monitored the strength of his flame? Instead of potentially burning it out. Fatigue is a major aspect of his condition and my window of observation was only one hour. But it was just one session, just one hour, what could the backlash really be?

I remember the times when it was just one shower, just 10 minutes, which had the consequence of many hours of bed rest for me. "Fatigue is one of the most challenging impairments to accommodate" (Wendell, 2001, p. 25). While being mindful of it, I have never made "pacing" an area of focus in sessions for people with fatigue as part of their condition. Yet fatigue, for me, makes the invisible map I use to navigate the days of the week. To ignore it, I would be lost.

Therapists may also mistake no observable changes as a sign of no progress or meaningful outcomes; changes can be internal, especially those on a spiritual level. Furthermore, some abilities may not be visible or audible, for example, a client may hum songs in their head, but this remains inaudible to the therapist.

Another effect is that music becomes an instrument of disability knowledge and control. One's music can become subjected to ideas about how it should sound when disability is reduced. For example, it becomes less perseverative or beating becomes more controlled and less sporadic, as in the evaluation of Nordoff and Robbins (2007), Creative Music Therapy. The music becomes disabled if it doesn't fit within prescribed Westernised norms. The experience of music-making as free and unrestrained can become subjugated when it is thought to represent disability. Aigen (2014) believes that buying into the notion of music as a mere tool, for observable non-musical goals, "inevitably leads to a dehumanised practice" (p.68).

\subsection{Expertise}

\subsubsection{Discursive formation}

Whilst discourses of observability allow anyone to determine whether someone has a disability or not, discourses of expertise place therapists, specialists, doctors etc., as the expert-knowers and most equipped to determine disability. As Samuels (2014a) states, "fantasies of identification operate on the level of the 'obvious', the 
'commonsense', yet simultaneously claim that only the expert can fully discern their meanings" (p. 3). The discourse of expertise relies on rationality/objectivity, fixed categories (pathology) and disability identifications that are negative framed. There is a lot of psychotherapy (and supervision) writing about blind and deaf spots. Referring to these disabilities as less than, not helpful to the process, and positioning them as examples of lacking in expertise.

The truth that competent music therapists should have special skills that determine illness/disability is endorsed by the NZ Standards of Practice. It states that we must "recognise and have critical understanding of methods of distinguishing between human health, sickness and disability, including diagnosis, symptoms and treatment" (New Zealand Music Therapy Registration Board, 2012, p. 3). Thus, it suggests that we can distinguish between these things with the application of certain methods. It doesn't take into account the lack of consensus regarding illness/disability definitions and different understandings. It instead implies that there are right ways to identify health/illness/disability: an object that therapists can capture. This understanding underpins the rest of the document that positions the therapist as expert with no inclusion of the client's expertise. Statements suggest that the therapist is in direct control over clients, using their understanding of illness/disability identification over someone else. This document shaped my earlier practice.

Going through old records I see that I have labeled someone's level of anxiety directly from what I have observed. For example, "highly anxious", "very anxious" or "their anxiety has reduced", even though one can be very anxious without showing any obvious observable signs or vice versa.

This belief that the therapist can directly access a client's experience of illness/disability is maintained through professional practices, including journal articles, conferences, and certain models. Disabled people themselves are less likely to be given the opportunity to have their voice directly heard, in whatever form that may be, which corroborates the expert-knower positioning. Due to the nature of their disability, one may not be able to contribute in traditional scholarly ways or use the 
language of expertise. Within this discourse, therapists/health professionals are positioned as experts by labelling illness/disability with professional diagnostic language in the dissemination of information, which is challenging for a layperson to understand. Examples I have used in past discussions of my work include stimming, echolalia, dysarthria and spasticity. An example of the authority of music therapists' voices is inherent in the Journal of Music Therapy, a forum for authoritative articles of current MT research and theory. The authors are music therapists or paraprofessionals. Furthermore, some professional practices render people as cases through individual disability markers. This further supports the position of expert as they become a "case" for the expert to solve and "upon which power is exercised" (Garland-Thomson, 1997, p. 40). Priestley’s pioneering Analytic MT model supports the belief that the therapist can access and understand the client's inner problems of conflict through symbolic music and the transference-countertransference process (Rolvsjord, 2010).

\subsubsection{Power}

Six power effects are highlighted below:

1. Therapists gain the authority to define

2. Therapists can distance themselves from disability

3. Disabled voices struggle to be heard whilst therapists are given platforms to speak

4. Disability is misunderstood with narrow understandings

5. Language used to sustain the position of expert dehumanizes

6. Dehumanisation can affect a person's participation in their own care/process

To demonstrate these six power effects, I start with my reaction to attending the NZ Hui in 2015. I have struggled to leave in the journal entry below, as I greatly respect the presenters for sharing with us all. I offer this not as a critique to their presentations and work, but as an exploration of the felt effects of the use of expertise discourses.

"I see you distance yourself, like the presenter before you. I see you do this, because I have dowe this. Client process highlighted. Therapist process erased 
$(2)^{15}$. Yes, it is about the client, yet who interprets the client? Parents speaking for children. Therapists speaking for clients. The therapist we know little about. Where is the voice of the 'absent special guests' (Todd, 2006)? (3). I was unsettled by this as I later ran my fingers along my own patient notes. I felt the incorrect statements: the ink marks me and doesn't scrub off.

You examine their reactions, struggles, moments of reaching. Where are your reactions, struggles, moments of reaching (2)? You watered them, what of the water they gave you? Equality lost. Shared journey becomes a journey of one. "We" becomes "them". The imbalance separates. But like Priestley, I am an us, I am a them (Bunt, 2004; Hadley, 2001). I am squirming uncomfortably and remain quiet.

I find myself at the hui, but I don't "find myself" at the hui. I see two cultures I belong to, disability and MT, put on display, but I don't see me in them (4). The word disability is said, but the meaning of disability is not talked about (2).

The disabled people have all become "cases" and "case studies" (5). I have made them this too, which I wrote about in my journal. "Sorry, I am unable to take on any new cases", I once said to an inquiring parent. Thirty seconds is how long it took to turn her child into a "case". Afterwards I drummed my fingers one after another on the kitchen bench while wondering whether I would know something of the child had I listened for longer and taken words like "case" out of the dialogue. Did he roll snails out of freshly baked play dough? Did he prefer the sound of his brother's drumming to the sound of his mother's piano playing? I will never know. If I had more information, would I have a better sense as to which therapist to refer him? Sterile terms like 'case' have a way of freezing conversations: anaesthetic for the tongue. My fidgeting fingers did not sooth my desire to go back in time and change that phone call. The outcome would be the

15 The numbers used in this journal entry are examples that correlate with the power effects with the same number above. 
same: I could not see him. But I would be referring on a child not a "case".

You see, I was once asked by a receptionist if I happened to know my NHI number. I replied, “yes it is BDK $5146^{16 "}$ " I am three-letters-four-numbers long when I walk through the sliding doors and stand on the polished floors of the hospital atrium. I am symptoms, tests completed, diagnoses given, and medications tried. I am the 'interesting case' or the 'complicated case'. My contextual life tries to squeeze in to one sentence; a passing mention of getting married. My symptom lists hide my experience from sight. I want to hide. My husband drags me to some appointments (6).

And so at this hui, I contemplate the language and practices that shut the doors for the people we work with to define for themselves and drive what disability and illness mean.

In discourses of expertise, the expert voice is granted platforms to speak (conferences, journals, reports) where the therapist's own subjectivity is not always questioned or disclosed. Therefore, presenting themselves as subjects of rational thought and reasonable action.

The belief that we, as professionals, are the most qualified to assess and describe disability has unsettling implications (A. Davis, 2005). In particular, expertise discourses overshadow one's own narrative of bodily and social identity. In Allan (2006), accounts from disabled students showed "highly sophisticated forms of transgression against the identities and the experiences that their teachers and parents imposed upon them" (p.282). Fairchild and Bibb (2016) ascertained that what children found important and their presentation in MT were not the ways in which family members, schools, and professionals described the children. Thin descriptions of people's identities are often created by professionals and presented as truths (A. Morgan, 2000). Gathering "evidence" to support these problem-saturated stories becomes easy once thin conclusions take hold (Morgan, 2000). Subjugated by the expertise discourse is the more recent view of disability as a personal and cultural

\footnotetext{
${ }^{16}$ Changed NHI number to protect personal information.
} 
identity where the individual defines for themselves their disabled identity and doesn't rely on the experts' interpretation or identification of it.

The expert's different understanding of disability caused significant misunderstandings. To them my disability was increasing because nerves were not regulating blood pressure and heart rate, there was an absent response during QSART and an absent pulse pressure during the Valsalva maneuver. She thought a wheelchair created disability ${ }^{17}$.

To me, I felt disabled by the lack of chairs in art galleries and shopping spaces. I thought the wheelchair lessened the disability. I no longer had to miss out on attending exhibition openings with my husband or abandon shopping missions with family smuggling me unconscious into the car.

Disabled people may be challenged to find representations in therapy journals and conferences that resonate. I and other patients search the internet reading patients stories, positive or negative, because they feel more real than the accounts written about us (Erevelles, 2011). Furthermore, the language used to sustain the position of expert, such as pathological terms and case studies, dehumanise the individual. Passivity in one's own therapeutic journey can ensue.

\subsection{Normality}

\subsubsection{Discursive formation}

A central feature of fantasies of identification is the medical framework that has penetrated into wider culture and influenced representation, policy, and more. It has only been possible for medicine to scan bodies in search of identifying characteristics with the social apparatus of normalisation (Samuels, 2014a). Developments like statistics and the concept of the bell curve in the early $19^{\text {th }}$ century produced the norm (Thomas, 2007). From this, the "normal individual" was developed by psychology

\footnotetext{
17 Wheelchair users are often described as being "wheelchair bound", due to the prevailing fear in society that wheelchair use is severely limiting and associated with significant disability.
} 
and medicine (Rose, 1996). The concept of disability as an abnormality to be normalised, coincided with the emergence of professions that have the remediation of disability as their goal (Straus, 2011). Disabled bodies were deemed inferior and marked with otherness while the unmarked sheltered in "the neutral space of normalcy" (Garland-Thomson, 1997, p. 8).

Discourses of normality are used in practice to identify the presence of illness/disability. Music therapists in NZ must "demonstrate sufficient knowledge about typical and atypical human development throughout the lifespan e.g. language and communication development, congenital and acquired disability" (New Zealand MT Registration Board, 2012, p. 2). I applied this knowledge when in 2009 a colleague and I devised an assessment sheet which we adapted from several sources including the Individualized Music Therapy Assessment Profile (IMTAP) (Baxter et al., 2007) and Dianne Langan's (2009) Music Therapy Assessment Tool for Special Education. It used a scale from 0 (never) to 4 (all the time) to answer statements such as "vocalises at appropriate volume", "stays seated in seated activities", "displays appropriate muscle tone in activities", and "makes eye contact with therapist". The statements and scale on the assessment form were based on what was normal and desirable whilst simultaneously reinforcing the desired norm. I used this for a year before concluding that it was not helpful. Similarly, the IMCAP-ND assessment tool developed by Carpente (2013) ascertains desired responses that, whilst not implicitly stated, are based on norms of relating and being.

Kenny (2006) expressed that observable levels of conformity, functioning level, and appropriate behaviour took precedent in the methods of therapy at that time: "Many therapists and methods presume they have all the knowledge about where and how a person must be healed. Many forms of therapy dictate exactly the way a person must alter their behaviour, attitudes, and mood, and conform with culture" (Kenny, 2006, p. 39).

\subsubsection{Power}

The discourse of normalisation in illness/disability identification has the following interrelated power effects that will be further discussed below: 
- Diverse ways of being become marginalised and invisible: Disabilities' "abnormality" status contributes to othering rather than being understood as another way of being.

- People with disabilities may hide their strategies for managing their disability to meet able-bodied/minded standards in order to be seen as less disabled/nondisabled.

- Incongruent observations of "normal" and "abnormal" create confusion and misunderstanding.

- The "abnormalities" identified influence how the person is understood.

- Music becomes limited to an able-bodied-minded aesthetic/standards.

The practice of normalisation, and the assumed essential able body, can make difference and other ways of being undesirable and invisible. People with disabilities are assessed according to conventional standards about what constitutes desirable normal behavior (Stone, 2005). Conformities are rewarded and departures from dominant expectations never go uninterpreted or unpunished (Garland-Thomson, 1997). This can result in them hiding strategies for managing disability to meet ablebodied/minded standards. For example, if I have to lie down when I'm out of the house, like Crow (2013) explains, "I seek out-of-the-way spaces: corridors and empty classrooms, fields and first aid rooms and once, even, a graveyard. I wait to be alone, tuck myself from sight and then, only then, as though it is a thing of shame, I recline" (p.359). Ros Blackburn described how she hides her autistic self, which is mischievous, quirky, and playful: she learnt to present a polite, restrained, and controlled image to the world (Prizant, 2015). Society teaches people with disabilities to deny much of who they are and suppress it (Kuppers \& Marcus, 2008). Hiding the disabled aspects of one's experience does not lessen them; it just makes them more invisible.

Furthermore, the slightest suggestion of normal function can create suspicion around the categorisation of one as disabled (Samuels, 2014b). Incongruous behavioral displays of normality and abnormality, for example, if a person in a wheelchair stands up to reach the top shelf, causes social confusion (Samuels, 2014b) and is the site of ridicule and disbelief. For example, the image below featured across social media sites in October 2015. 


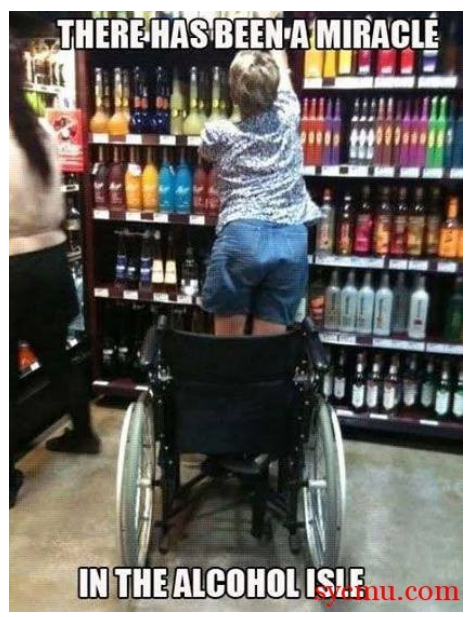

[Meme of a person standing up from their wheelchair to get a bottle of alcohol. The caption says, "there has been a miracle in the alcohol isle"]

Examples like these are plentiful, such as the news heading "Federer's miracle shot helps man rise from wheelchair" in 2016 suggesting how disabled people should look and act.

When we can't tangibly see/feel what is causing a particular behaviour, one's actions can be labeled as deficits or problems (abnormalities) instead of useful strategies and reasonable responses. For example, traditionally people with autism were trained to give eye contact, even though, as they describe, it pains them to do so. This practice is becoming less popular. Similarly, the repetitive behaviours seen in people with autism have been labeled as stimming, problematic, and in need of reduction. As Prizant (2015) explains, their efforts and creative ways of managing what we cannot directly experience are disrespected and shamed if the goal of therapy is to give eye contact or to stop stimming. The discourse of neurodiversity interprets stimming as productive, communicative, and pleasurable and is embraced as a manifestation of difference not a symptom of deficit (Bakan, 2014). Furthermore, author and scholar Walker (2014), who himself has autism, contends that autism has often been misconstrued as being a set of social and communication deficits: autism is actually a by-product of the intense and chaotic nature of sensory and cognitive experience that occupies the person's attention and energy, meaning less energy and attention is available to focus on the subtleties of social-interaction. This interpretation is compounded when social rejection occurs because social expectations aren't met. 
Additionally, when someone is unable to find a strategy to manage an invisible disability, it can be the source of disciplinary action or blame can occur because they look normal.

I watched as a student was told off for not doing something they had forgotten to do instead of being offered tools that supported them to manage their memory difficulties (invisible disability).

A tree was blamed for moving instead of acknowledging the wind.

It's sticks and nearby stones lay innocently still.

It was words that did the breaking.

To have one's strategies labelled "abnormal" loses the humanness of the response. The support teacher said, "he has been stimming all morning, I couldn't get the lids he spins off him sorry-so they have come to music". "That's ok" I replied, "He can have the lids here". I shake my leg when I'm watching TV: it's soothing. I listen to the same play list repetitively as the infusion pump churns: it's calming. I wonder about the different understandings between his "stimming" and my self-soothing: One considered negatively, one positively (Prizant, 2015).

The dominant ideology pathologises people with disabilities (A. Davis, 2005) and they are defined by what they cannot do, as opposed to non-disabled who are defined by what they can (Swain \& Cameron, 1999). When disability is foregrounded, it is expected to explain all life experiences of the individual (Erevelles, 2011). Problems and abnormalities are related back to the individuals impairment (Levinson \& Parritt, 2006). The illness/disability becomes what Goffman (1963) described as a master status, the attribute that colours the perception of the entire person and its ability to neutralise positive qualities. For example, there is no certain way of knowing from observations whether someone's lack of active participation in MT is due to a personality trait or an underlying disability. I have heard many accounts of a person's lack of active participation at the beginning of therapy being abnormalised. Having 
one's personality attributed to one's disability is part of the disability experience (Stanford Encyclopedia of Philosophy, 2011). An effect of this is, as Grant (2011) describes, that reducing me to my disease, reduces the therapy. This is because reductionist understandings impact on how people are treated in the flesh.

The discourse of normality, and its use in disability identification, also influences what we think good music is. Westernised normative music standards can be used to refer to different states of wellbeing or pathology (abnormality/normality) when understood from an able-bodied/minded perspective. Ferris (2011) wonders what it would mean to live in a world that understood a different aesthetic. A disabled aesthetic that appreciated other ways of being. Non-disabled or hearing world perspectives are largely used in describing the music of people with disabilities or deaf individuals (W. Churchill, 2015). There is literature outside of normalised medical discourse that describes an autistic musical culture based on music that pays attention to discrete musical events, private associations, and more (Straus, 2014). However, Deaf musicians, such as Evelyn Glennie, became successful when they found ways that permitted them to conform to audiocentric normalised expectations (W. Churchill, 2015). This is not possible for everyone. Furthermore, it can normalise one out of their cultural frame instead of seeking to enhance it (Straus, 2014). Limitations on what we consider to be musical affect the inclusivity of all human bodies making music and alternative music aesthetics.

When I was first unwell, I felt frustrated and inadequate when confronted with situations with normalised standards of music making. My guitar playing became "below standard" as I played it lying down and I could never last the full length of choir rehearsals.

But I kept starting compositions (short, fragmentary or full of silence) that I never finished. I had the intent to play rhythmically but was like a wind-up toy that slowed with time. I opened YouTube to find new songs, but like a stuck record I listened to the same thing over and over: Finding something new in the rePeat, Repeat, repeaT. Ultimately, I found a new aesthetic from the 
incomplete, arrhythmic, repetition that complemented my way of being ${ }^{18}$.

\subsection{Ethical considerations}

The idea that illness/disability are easily identifiable raises ethical issues around the disabled voice and representation in practice. Definitions of disability, based on discourses of observability, expertise, and normality, can conflict with and invalidate the disabled person's understanding of their own disabilities (Kielhofner, 2005; Scott, 1969). One consequence of professionally-driven understandings is that "a core element of the experience of disability is being seen as something you are not, joined with the realisation that what you are remains invisible" (Gill, 2001, p. 365). As a result, disabled persons can find themselves "prisoners of the misconceptions of others" (Gary \& Hahn, 1997, p. 395; Kielhofner, 2005): the way they are described can influence how we are treated in the flesh (Grant, 2011). Narrative therapists draw attention to how human problems occur when "the way in which people's lives are storied by themselves and others does not significantly fit with their lived experience" (Carr, 1998, p. 486). The above analysis raises the pitfalls of trying to define the reality, interests, needs or experiences of another group or person (Baines, 2013; Mullay, 2001). It suggests that regardless of the level of disability, wherever possible, it might be beneficial to consider and create opportunities for disabled people to influence decisions (Rolvsjord, 2010), and to express an opinion about what they have done and ongoing plans that affects their life (Rickson \& McFerran, 2014). Each person is the source of their own health/disability experience and, therefore, the creative author, because health and disability cannot be prescribed by societal norms (Lindsey, 1996; Parse, 1990). Such a collaborative co-authoring practice parallels narrative therapy (Carr, 1998; Epston, 2016). If we understand that the identification of an individual as disabled does not only involve the use of purely physical or objective standards, but also a range of socially determined norms that direct policies and institutions, it helps us to see why it is a mistake to suppose that the determination of whether someone is disabled is a purely factual or simple matter (A. Davis, 2005).

18 An example and further discussion are given in chapter 9. 
Such assessments that we do as therapists have a "verdictive character" (A. Davis, 2005, p. 157).

The ethical issues related to the disabled voice and representation in practice raise an ethic of subjectivity. In such an ethic, therapists would reflexively critique views and actions that create the disabled subject, and thereby recognise their own subjectivity in the formation of therapeutic knowledge. Reflexivity destabilises the professional as a rational human being describing objective truths about clients. Ethical subjectivism teaches that there are no objective moral truths. Without acknowledging our subjectivity "we run the risk of merely controlling the patient or fitting the patient into a mould, which we as therapists consider ideal, or which we as citizens of a society consider responsible" (Kenny, 2006, p. 134). This ethic counters the objective expert position. MT research methods such as participatory action research (Rickson, 2014d) and approaches such as, anti-oppressive MT (Baines, 2013), community MT (Ansdell, 2002), ecological MT, the field of play (Kenny, 2014) and feminist MT (Edwards \& Hadley, 2007), all supplant the expert position and therefore offer some ways of working in an ethic of subjectivity. To counteract the normative and observational truth-making that disability is easily identifiable, which I encountered in my earlier practice and work contexts, there is a need to consider how the ethics of subjectivity might reframe some of the NZ code of ethics and standards of practice for music therapists. As noted, to date (2018), statements within these documents are still based around disability/health being identifiable by the therapist. Subjectivity offers potential for developing alternative understandings. 


\section{Chapter 5}

\section{Disclosure: To Hide or Not to Hide in the Box?}

"Hiding": Photograph taken during a session at the request of the student

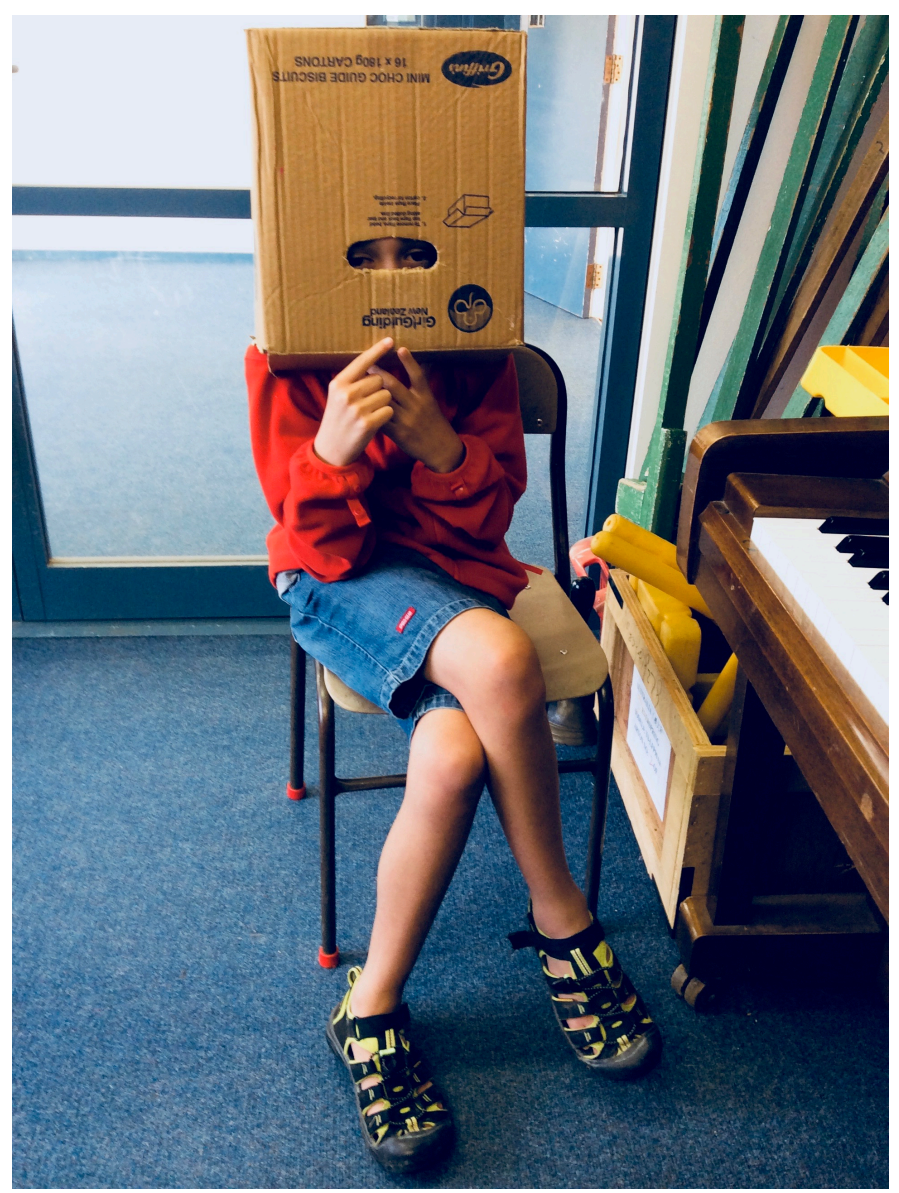

[A young primary school student with a box on their head and eyes peeking out through the hand hole.] 


\section{Introduction}

"Marcus, see his eyes. He has no mouth. No nose. No hair. Hiding somewhere else" (Marcus's song lyrics).

He came to music with a box on his head, only his eyes peeking out. Above were the lyrics he wrote for his Box Head Song. I want to know where else it is he's hiding. Society teaches people with disabilities to deny much of who they are and suppress it (Kuppers \& Marcus, 2008). I hope Marcus can be whatever self. I ask him if he would like some string to secure the box or if he would like to take it off. I later ask myself the same question.

It is not just the people we work with who can grapple with how to present themselves and what to disclose. Due to the "truth" discussed in the previous chapter, that illness/disability are easily identifiable, if there are no observable signs nondisabled is assumed unless otherwise stated (Swain \& Cameron, 1999). An effect of power derived from discourses of disability obviousness is that it creates an environment of disclosure/non-disclosure. Disclosure was a large issue to explore and warranted its own chapter. There are multiple factors that drive issues around disclosure. In this introduction I will discuss some of the wider social/cultural conditions as well as some possible positions available for people with non-apparent disabilities that result from "passing privilege" and "passing disadvantage".

There are many social factors that may incite and pressure the person to pass as nondisabled (Swain \& Cameron, 1999). The term "passing privilege" recognises the benefits obtained by people when culturally accepted markers for disability are missing, such as in invisible disabilities. By passing, the person can avoid potential prejudices and discrimination that can exist towards disabled people. For example, disabled people are over-represented in unemployment and benefits, and disability discrimination has been the most common employment complaint received by the Human Rights Commission (CCS Disability Action, 2013). Negative discourses around disability continue to be used in New Zealand (NZ). In the media, disabled people are presented as victims or heroes, rather than people doing everyday things (Stace, 2007). These affect attitudes towards disabled people, which can result in pitying comments, infantilisation, condescension, and patronising behaviour within 
interactions (Wendell, 2001). Disclosing can therefore result in losing the privileges non-disabled people have in not encountering such discrimination and attitudes.

However, those who contend that the invisibleness of a disability does not mitigate its impact, and that there is similar rejection, humiliation, and social disapproval towards those with invisible disabilities, disagree with the term "passing privilege" (A. Davis, 2005). The certain level of privilege from passing can come with profound internal dissonance and a sense of misrecognition (Samuels, 2013). Wall (2005b) comments that she passed at times to blend in and be less alone, which made her feel more alone in her inner world. For her, there was no privilege in this isolation or alienation from the self. Some people with invisible disabilities opt out of situations where they would experience difficulties without giving a real reason in order to hide their limitations, increasing isolation (Stone, 2005). For others, access to resources and accommodations can be hard to obtain due to their normal appearance. When a person's body does not match identifying disability markers, they are open to scrutiny and can be unfairly blamed for faking their disabilities in order to gain special privileges (Samuels, 2014c). Such accusations of being illegitimate claimants to disability status invalidate one's experience and are a painful indignity (Green, Davis, Karshmer, \& Marsh, 2005). Furthermore, there is often a physical cost when one has to use every inch of strength to pass: for example, fatigue occurring after standing for too long. Unrealistic expectations can be placed on a person when their disability is non-apparent, leading to their difficulties being attributed to laziness. This contrasts with people with visible impairments who are often considered incapable of things they could easily do, which is equally problematic. Therefore, the position of "passing" to obtain privileges also comes with, what I would call, "passing disadvantages".

A dominant discourse in disability studies explains disability as social oppression. Within this discourse not identifying as disabled, non-disclosure and passing, positions the person amongst various forms of oppression and is understood as a form of self-oppression (Swain \& Cameron, 1999). In social oppression discourses, 
"coming out" is a process of rejecting the tyranny of the normate ${ }^{19}$ with an identity that does not need to be hidden; instead disability becomes a social category that needs to be challenged (Swain \& Cameron, 1999). Making difference visible, Siebers (2008) argues, has the capacity to challenge and undermine cultural norms and processes of stigmatisation (LaCom \& Reed, 2014; Siebers, 2008). By trying to appear to meet able-bodied standards, other aspects of our human paradigm can become neglected/subverted (A. Davis, 2005). Within this discourse, if I allow the cultural gaze to categorise me as "normal", it raises the question, "am I in league with the ableist agenda?" (Pafunda, 2011, p. 314). Choosing silence holds up an inequitable system and submits to the notion of better treatment being found in the dominant group. It demonstrates that not all identities are good to have. This discourse offers those who self-identify as disabled a more empowered position, while those who don't are offered a position of being oppressed and/or a contributing oppressor.

Some have undermined the claim that passing as "normal" is by definition a form of negative disability identity (Samuels, 2013). Instead, those who pass are skillful interpreters of the world, not cowards or con-artists (Siebers, 2013). They adapt their position based on whether a context feels safe or not to disclose in. The body itself can also influence non-disclosure: "For all the times I've tried to explain this illness to others, there have been just as many times I've felt too tired to make the effort" (D. Wall, 2005, p. 12).

These social conditions, and the benefits and detractions of different positions, contribute to the dilemma around disclosing ${ }^{20}$. Indeed, one might choose to "embrace, reject, conceal, or reveal a disability" for multiple reasons (Olney \& Brockelman, 2003, p. 35). The issues are complex, multifaceted, and full of contradictions.

\footnotetext{
${ }^{19}$ Rosemarie Garland-Thomson (1997) coined the term "normate" to describe the veiled subject position of cultural self that is knowable from the deviant others that mark the normate's boundaries. The position emerges when we examine and critique the social processes and discourses that constitute otherness.

${ }^{20}$ It's important to note that not all contexts create issues around disclosure, some are more disabling in this way than others. In some work contexts I feel comfortable to disclose, whilst others I am less inclined to do so.
} 
Throughout my day I navigate disclosure across the different contexts I encounter. The contexts have different, although sometimes interrelated, constructions of truth, power relations, and ethical issues. This chapter will be structured by, and divided into, three different contexts that I am faced with disclosure as a music therapist with a illness/disability: 1) university, 2) supervision, 3) music therapy (MT) practice. In the spirit of disclosure, each context starts with a poetic letter illuminating what was said, done, and thought. Following each will be an analysis of truth and power that relate to the discourses and experiences described in the poetic letter. Ethical issues raised will also be discussed. 


\section{University}

\section{Journal:}

Dear university students and staff,

I have to do this course,

I'm not welcome to just sit in.

I must do it "like the other students".

To get the reading list.

I read between lumber punctures,

Cystoscopy and CT.

I can't do it "like the other students",

If my body absences me.

I can't get the requirement waived,

Because another student didn't.

I must do with her another course,

To be granted confirmation.

I have to present my proposal,

I put foundation on

To look just "like the other students"

To get an approving nod.

Upright it was hard to form answers.

I shivered for half an hour after,

I climbed stairs away from the examiners,

And recovered on the carpet. 
Others' watched my presentation,

They said I did just great,

But thought it was too much like the other students,

My struggles did not translate.

It's a struggle to be yourself,

in a place comparing you to others.

It's a struggle to be like the others,

Loosing hours under covers.

This is why I am writing, for it's something you should know:

That in this environment currently,

If I'm real or if I hide.

The face presented of me

Was a struggle I survived.

\subsection{Truth}

At Victoria University of Wellington, the discursive truths of fairness influence disclosure. The particular "truths" that dominate within this educational discourse are that fairness is required to measure student achievement and therefore located within individuals based on the normative of non-disablement. To begin this discussion, I note that for students with impairments, fairness considerations are based on ambiguous wording. Kia Orite, is a code that was written to make higher education more equitable for people with disabilities throughout NZ (Achieve, 2004). It was a landmark document with significant importance in assisting universities in disability inclusion. In this code, an equitable learning environment is one where "reasonable accommodations have been made to enable students with an impairment to participate 
in a course" (Achieve, 2004, p. 62). Interpretations of what is "reasonable" therefore rely on ideas around fairness. This section discusses some issues Kia Orite raises.

Fairness within Kia Orite is related back to and compared with non-disabled students. It explains accommodations as "adaptations that remove barriers to enable equal participation. These are based on the premise that students with impairments should be neither disadvantaged nor advantaged relative to other students" (Achieve, 2004, p. 62). An example of fairness being placed relationally between individual students can be seen in the course outline of a paper initially required for my $\mathrm{PhD}$ that stated, "in fairness to other students, unless a medical certificate is produced, assessment submitted after $5 \mathrm{pm}$ on due date will be subjected to a $5 \%$ demerit on your grade, increasing by 5\% each further working day" (New Zealand School of Music, 2013, p. 3). This example of "fairness" is based on dominant non-disabled standards. For someone usually well, going to the doctor when sick and getting a medical certificate is a typical course of action. In the context of chronic illness, going to the doctor to get a medical certificate due to a flare in symptoms can be less reasonable when it is typically managed at home. For courses where assignments are weekly, multiple unnecessary doctors' visits may ensue. This also creates an environment where one has to continually get their disability validated to prove that accommodations are fair.

It is interesting to note that "fairness" comes into question in these documents when a student requires a change to be made or does something differently. Therefore, "typical" students are less likely to be questioned as fair and reasonable: if the student with an impairment discloses to obtain accommodations, fairness is then considered. It is administrators, lecturers, and heads of school, that determine what is fair, not the student with the disability.

Kia Orite stipulates that reasonable accommodations allow students with impairments to compete on equal terms with others. It doesn't acknowledge that due to the nature of some impairments, they may not be able to compete on equal terms even with the removal of barriers. This idea-that if barriers are removed (such as addressing inaccessible buildings) disabled people will perform the same as everyone else-comes from early social models of disability. In some ways this position tries to satisfy demands for normativity (Erevelles, 2011). In the case of chronic illness, asking for accommodations of pace and time threatens the message that we can perform as 
everyone else if barriers are removed, because studying to the university's course schedule is usually considered an aspect of student performance (Wendell, 2001). Efforts designed to remove obstacles for full participation and "fairness" are often based on the idea that bodies are permanently and predictably impaired (Wendell, 2001).

This aspect of not performing like everyone else when environmental barriers are removed is not considered in what is "fair".

An example of this can be found in the personal experience drawn on in the poetic letter above, where despite asking for a flexible time schedule for assignments, to accommodate unpredictable symptoms, it was initially denied based on needing to know the same material at the same time as the other student for discussions. Getting all of the assignments at the beginning of the course was not possible either, which meant that the management strategy of getting ahead in times of wellness and taking breaks when unwell was not available to me. I needed to perform like the other student.

\subsection{Power}

I will discuss four power effects relating to the truths discussed above:

- Increased bureaucratic exchanges

- Disempowerment

- Financial/physical cost

- $\quad$ Safety

Students with impairments have more bureaucratic exchanges (Morella-Pozzi, 2014), which is part of the surveillance by others to ensure fairness. This might include, supplying a letter from a specialist or doctor to validate the disability when registering with disability services or disclosing to staff; meeting with disability services each time an accommodation is sought; form filling; following up on accommodations that have not been implemented; and email exchanges. The powerful effect of this on students with disabilities is that sometimes it is easier to forgo seeking 
accommodations to spare oneself the energy and time involved trying to jump through hoops.

The advocacy of disclosure has a place, but identifying as impaired still seems to offer a more disempowered subject-position that can be de-authorised and rendered voiceless (Claiborne, Cornforth, Gibson, \& Smith, 2011). As students with impairments have to seek accommodations through university staff, it can create a relationship of helper (staff member) and helped (student with an impairment). Furthermore, those who are positioned to grant less restrictive university environments or create them determine what is fair, even though the needs of the disabled student might be different from those making decisions around how their education is delivered and knowledge evaluated. Disability services have an important role in supporting students to obtain accommodations, but they also do not have a say in final decisions regarding what is fair. When necessary, complaints are to be dealt with using mediation. Legal proceeding can occur in serious cases (Achieve, 2004) but require time and resources to pursue.

Accommodations given are ultimately up to the university department/lecturer. Different university departments interpret reasonable accommodations and fairness differently, and therefore govern themselves accordingly. Such discrepancies are influenced by decision makers subjectivities.

Within a different department they felt it was reasonable for me to "audit" a research paper, without having to attend all classes and complete assignments-to simply gain access to necessary knowledge from which I could self-direct learning from home. This was not considered reasonable in another department.

With such inconsistencies, it becomes difficult to decide whether to disclose the reality of one's impairment; it might be helpful in one circumstance and disempowering in another.

Further strain can be placed on financial and physical resources, which may already be overstretched by significant ongoing medical costs and managing symptoms, in order to ensure the fairness of others, such as paying for the required doctor's certificates for delayed assignments. Physically travelling to the doctors, waiting, and 
the consultation can also deplete energy, increase symptoms, and delay recovery time, with no benefit of a different treatment plan.

Discrimination based on ideas of fairness can make the environment unsafe. A culture of disbelief and skepticism can occur, specifically towards those with non-visible disabilities, when exceptions from normal standards are required (Fraser, 2007; Morella-Pozzi, 2014). Hadley (2017) states that "music therapy students with disabilities have been told that they are 'exploiting' their disabilities to their advantage by 'using' their disabilities to get them out of doing hard work" (p.39). When accommodations of pace and time are perceived as unfair they can be met with resistance from other students and staff due to the politics of resentment (Wendell, 2001; I. Young, 2000). Having a disability can be seen as being inconvenient and unfair on others as they have to bend the rules (Morella-Pozzi, 2014), fostering the attitude, which still remains, that the disabled ask for too many favours (Weise, 2011). Indeed disabled MT students are complemented when they do not need accommodations, thus communicating that they are more fair and acceptable when they appear closer to the norm (Hadley, 2017).

This can impact on disabled students governing themselves to be good, fair or easy going in the eyes of others, sometimes at the expense of what is helpful for them.

I was aware that not adhering to the stereotype of uncomplaining victim, can lead to being identified as another - bitter, unfair (Swain \& Cameron, 1999). My supervisor strongly suggested that we ask the school for a suitable environment for my confirmation event (a warm room with easy access). I down played the need for this, to my own detriment, because I didn't want to be a bother when it was the only option the organiser could come up with. In another instance in the poetic letter written above, I felt like a nuisance for even asking for the reading list, and wondered if I was viewed as demanding, so I stopped asking for it. I would look at myself through the eyes of others (Du Bois, 1999) to present the self I thought they wanted to see.

Within some university relationships/contexts it was easier to resist the power effect of feeling unsafe and the "need to pass" was not felt. For example, it 
felt safe to disclose within PhD supervision (as this was a flexible, adaptable, and responsive space to any issues/barriers), but it felt significantly less safe within other university contexts.

In the poetic letter above, my struggle with how to govern myself is evident. I would rather not mask my disability, but I did not want to deal with potential discrimination (Poulin \& Gouliquer, 2003). I sat before three examiners I had never met. Hence, I managed the sharing of my information on a "need to know basis" so I could demonstrate my competence first. Even now, I prefer to wait until it feels safe (Matthews, 2009; Olney \& Brockelman, 2003).

\subsection{Ethical considerations}

As disclosure remains an issue within the current fairness discourse, considering an ethic of diversity might be beneficial. Whilst Kia Orite has helped to address inclusion, it has not specifically had a major impact on issues around disclosure (Claiborne et al., 2011). Students may feel like they are damned if they do and damned if they don't disclose, with structural fairness not adequately addressed for diverse ways of being and the lack of a safe environment.

How fairness has been constructed in this particular university discourse enables the status quo of course delivery and assessment to remain in place, as it does not address the disabling structures that exist. It is, as Rix (2012) points out, that equality becomes a screen behind which the replication of inequality is hidden when dominant social groups maintain existing conditions while appearing to support those they marginalise. This continues because while opportunities for higher education are now available, institutions rely on the medical model of normalising or accommodating the needs of individual rather than making the environment more universally accessible for diverse ways of being (Humpal, 2017). 
As stated in a research report undertaken at Victoria University of Wellington by Claiborne, Cornforth, Gibson, \& Smith, (2011), "there was some consensus among students with impairments that the need to disclose might be mitigated by a 'safe' environment, flexible and adaptable courses offering a range of possibilities, allowing people to make the choices that suit them" (p.519). This requires the focus of fairness to be moved from students within existing structures, to assess the unfairness of structures that currently support how knowledge is gained and assessed: this could possibly reduce the need for and issues around disclosure. In short, attention to individuals accessing what already exists needs to be refocused to changing what exists to better suit diversity. The ethics of diversity is one that acknowledges both the difference created by impairments and the removal of social barriers in creating a fair environment for diverse ways of being.

As it stands, students with impairments can be treated differently if it achieves equality. If learning environments were adaptable and flexible for all students, there would be no or less need for different or "special treatment" for students with impairments and therefore less need to disclose. Policy and courses based on meeting diverse human needs might eliminate "special needs" with no negative shadow cast (Stanford Encyclopedia of Philosophy, 2011). From this analysis, it seems that we are not yet in such an inclusive situation that embraces diversity at structural levels. I am reminded of my difference. I sometimes feel I do not belong (Reeve, 2006). 


\section{Clinical supervision}

\section{Journal entries:}

Dear Mara (new MT supervisor),

I take a little step backwards on the first count of our waltz. With a new dancing partner I am unsure again, scared of moving incorrectly - waiting to be led. But you wait. Wait for me to create, as I hesitate in showing you some unruly steps: For I do not know your view on the movement of a body that cannot regulate its self, but uses it to support someone else's. So I limit the discussions of my patient narrative to the practical management of letting go of work hours. Alerting you to the fact that I am aware of my limitations and getting in first calling shotgun on, "I told you so". Self-critique as self-defense. I talk about my experience of illness in relation to the work I have let go (job $\times$ had too much driving), but not at appropriate times for the work that I have. I left out the ways it informs and colours, the helpful aspects that guide me at times. It forms a rainbow with an elusive end.

I tip toe around it, looking for something to pull me in to stand in the centre of my experience, but what I look to for help makes the thought more discomforting. I know the code of ethics says that "therapists must maintain adequate standards of physical, emotional and mental health and seek help, or refrain from working with clients if his or her fitness is impaired": A statement open to multiple interpretations of what adequate is. Is your interpretation mine?

I know that the longer I bite my tongue, the sorer it will be to talk. I am writing to know what dance moves are safe, that I am safe.

Can we waltz around the full landscape of my work?

Dear Mara,

Thank you for discussing with me my question. You had wondered whether the 
activity example you gave me, with the therapist standing with the drum, was something I could do? Had it given me the impression that to be a good therapist I must stay standing, you wondered? We had both been wondering instead of asking. In my mind I had already adapted that activity: a hallmark of disability culture is finding different ways of doing things (Ferris, 2011). It was not that your standing example made me feel like an inadequate therapist; I simply felt that I wasn't the therapist you had in mind. I had drawn you the wrong one, because I'm not the therapist I had in mind either. What pictures would our minds think in if we were taught that the disabled were therapists, instead of only in need of therapists?

Let's stop waltzing and improvise; I'm ready.

\subsection{Truth}

Supervision is required in NZ to "safeguard the quality of service to clients" (Music Therapy New Zealand, 2012, p. 2). It is a supervisor's role to assess and confirm during the yearly registration process that the therapist is competent and adhering to the Standards of Practice (SoP) and Code of Ethics $(\mathrm{CoE})$ for the provision of MT. Due to this, these two documents have been analysed to see how "truth" has been constructed and how it may impact on disclosure in supervision. The following discussion is based on three "truths" that have been identified in these documents:

- Disability is primarily a biological/psychological problem residing in the individual that needs to be reduced.

- Disability is something identifiable by others (not self-defined, not a selfidentity).

- There is an adequate level of health required to practice

The SoP are strongly framed by the medical model that understands disability as a result of a physical condition intrinsic to the individual. The individual client is at the centre for standards of assessment and "intervention". Identifying social barriers and the removal of these (such as inaccessibility to music making) are nearly absent. For example, statements such as therapists must "understand relevant client conditions and the application of music as a treatment option" (NZ Music Therapy Registration 
Board, 2012, p. 3) are common whereas sociological and ecological knowledge bases are scarcely mentioned. The SoP call for "sufficient knowledge about typical and atypical human development" (p. 2) and in-depth information about the client's condition, for example effects of medication. However, it does not also ask that therapists have sufficient knowledge in different understandings of disability or social, cultural, environmental conditions that shape how we view and approach disability. The "truth" formed, that disability resides in the individual and needs to be alleviate/reduced, has developed within the broader NZ history where the classification and labeling of the type of impairment has been more important than removing social barriers (Stace, 2007). Due to the predominant medical framing, disability culture, such as Deaf culture, is not specified as something to be aware of within the cultural competence section. This omission and lack of recognition of disability cultures seems significant when therapists predominantly work with disabled people.

The SoP construct disability as something identifiable by others and imposed by someone else, instead of it being an identity and condition that is self-defined. For example, the SoP state that therapists must, "recognise and have critical understanding of methods of distinguishing between human health, sickness and disability including diagnosis, symptoms and treatment" (p.3). Furthermore, while the document makes mention of the therapist's culture and awareness of this, disability is constructed not as an aspect of the therapist's identity (along with gender, sexuality etc.) but as a condition that is bad and potentially dangerous if a certain health standard is not met.

The CoE's suggests that there is an adequate level of health required to practice safely: "therapists must maintain adequate standards of physical, emotional and mental health and seek help, or refrain from working with clients if his or her fitness is impaired" (p. 2). This fitness standard statement is vague and left up to the interpretation of the supervisor and their ability to distinguish illness/disability as mentioned above. Within the context of these documents it is reasonable to anticipate that a supervisor's understanding might rest within a predominantly medical frame, which may not match the therapist's understanding of her illness/disability. Interestingly, the CoE singles out illness, and consequently illness related disability, in this respect. It does not state, for example, that a parent must manage home and 
work life adequately and refrain from working if home life becomes too demanding. One possible reason that illness/disability are specified as potential risks to ethical practice over other life events is the different truths that predominate in society around different situations. The struggles of parenthood are socially acceptable, but illness/disability are less socially palatable, often feared and considered tragic. Adequate health levels are also indicated in the larger global context of MT. For example, Bruscia's (2014) book defining MT states that therapists can only help clients if they preserve and protect their own health.

\subsection{Power}

I will discuss two potential power effects related to the truths constructed above for disabled therapists:

- Internalised negative self-identity/shame (stifling professional growth/understanding)

- Financial fallout

The prevalent use of medical discourse can create a situation where therapists hide their disability or illness due to the shame associated with locating the "problem" solely within the individual and it being framed as "social deviance" (Thomas, 2007). Therapists with disabilities may internalise ableism and stigma that results from this, leading to non-disclosure or discussing only the negative implications on practice (as I did initially). The transformative aspects of the disability experience may not be recognised and therefore stifle/delay professional growth. Because supervisors are working within the same practice guidelines and ethics, their reactions towards disabled therapists might be based on this medical model, which reinforces the stigma. Fortunately, I did not feel that that was the case for me when I fully disclosed. However, I note that in interactions with colleagues and within MT, adherence to the medical model is seldom made explicit, but is still a pervasive influence (Levinson \& Parritt, 2006; Rolvsjord, 2010). Considering that some illness experiences trigger deep transformation in the person's life and the task of modifying who they expected to become can be difficult (Fleischman, 1999; McCullough, 1989), being surrounded by medical discourse at work creates further challenges. 
Interestingly, Stanley et al (2007), found that many allied health professionals who had disabilities themselves did not want to be labeled as disabled. This is not surprising given that our culture offers profound disincentives and few rewards to identifying as disabled (Garland-Thomson, 2013). “The terms 'disability' and ‘disabled person' were perceived by many as being the most stigmatising... and professionals associated being a disabled person with 'feeling inferior', 'you aren't able', 'not full human beings', or being 'one of the weaker members of society' " (Stanley et al., 2007, p. 41). This exemplifies how working within this predominant discourse can cause practitioners to internalise ableism and stifle new meanings of disability or self-definitions/understandings (or invalidate them). As Garland-Thomas (2013) explains, "people refuse to identify with being disabled in part due to a lack of ways to understand or talk about disability that are not oppressive" (p.347). Positive signification of disability becomes unspeakable in the dominant discourse: "Disability can't be thought of/spoken about on any other basis than the negative, to do so, to invoke oppositional discourses, is to run the risk of further pathologisation" (Campbell, 2008b, p. 3).

Because disability and wellness have been constructed in these documents as something others can identify, disabled therapists are put in a passive position where others can distinguish their disability for them, further limiting the potential for selfdefinition/understanding. Supervisors are positioned to recognise, distinguish, and define whether a therapist has a illness/disability, to what degree, and its impact on their clinical work within supervision. However, this is an impossible position to maintain. Supervisors may instead find themselves in the position of not knowing. As mentioned in the poetic letter above, my supervisor acknowledged that she was unsure, which helped me trust that we could create our own understandings about my disability in relation to practice. This had a really positive impact on the way I was able to use supervision.

There is the possibility of financial fallout for disabled therapists. Because fitness standards are vague and non-specific across multiple disciplines (Stanley et al., 2007), they are open to interpretation. Supervisors' interpretations have significant implications as they must sign off on registration forms to validate competence. Money/salary can be one of the power related issues. If a therapist doesn't get registration, on the basis of fitness standards, then they cannot work as a registered 
music therapist. Hadley (2017) points out that some disabled people have been kept out of the profession as their impairments have meant that they cannot meet certain competencies and accommodations have not been given. Illness/disability may impact on how others' perceive what a therapist is capable of, which can affect promotion and job offers/possibilities. Therapists with disabilities have the ability to draw on disability discrimination acts should they need to, but these are often "not as effective for those with unseen disabilities, as the adjustments required are often attitudinal ones rather than physical adjustments" (Stanley et al., 2007). Therefore, disclosing can open one up to potential job discrimination and loss of income if there are not adequate anti-discrimination procedures in place.

\subsection{Ethical considerations}

The "truths" and their subsequent power relations create ethical issues around professional exclusion, oppression and discrimination. Discrimination based on negative understandings of disability creates an environment for non-disclosure and internalised ableism, which may impact negatively on practice. There is a need to take professional exclusion seriously as this can reduce the diversity of the profession. We need to be able to draw from people who bring diversity to our field (Boggan et al., 2017, p. 386) to ensure practices are representative of the diverse range of people it serves.

$\mathrm{NZ}$ practice guidelines do support a supervisory relationship used in such a way that "it provides a place where difficulties, challenges and uncertainties can be shared, discussed and worked through such that the supervisory relationship provides a safety net for clients and therapist" (NZ Music Therapy Registration Board, 2012, p. 5). But it needs to be acknowledged that supervisors are also practicing under these documents, which as demonstrated above can create a feeling that it is unsafe and a risk to disclose illness/disability. Safety nets against discrimination for therapists with disabilities, for example, are lacking. The professional board of representatives themselves are not subject to an audit concerning their fitness to include disabled people/people living with illness (Stanley et al., 2007).

Whilst there are times when a therapist with an illness/disability may be best to refrain from practicing for a period of time, this-like other life experiences such as, parenting, moving to a different country, relationship separation, bereavement, caring 
for elderly parents-could be well-accommodated under practicing self-care. Only marking illness/disability as a potentially dangerous state in which to practice creates othering instead of inclusivity as one of the many human experiences that can have implications for practice. There is a large body of evidence that shows how fitness standards are perceived and the fear of exclusion creates a barrier to disclosure (Rooke-Mathews \& Lindlow, 1998, as cited in Stanley, 2007).

In summary, like the previous section, this analysis calls for an ethic of diversity that supports diverse understandings of disability and therapists with diverse abilities and identities. Drawing on and including discourses such as anti-oppressive MT, feminist MT, and queer MT, within the NZ CoE and SoP would be advantageous. This may go some way in countering the potential of professional exclusion, oppression and discrimination created by medicalised individual deficit discourses that still govern $\mathrm{NZ}$ music therapists' practice, reducing issues around disclosure. 


\section{Music therapy practice}

* Journal entry is indented

Dear Sara,

In this room I transform.

I become who you need me to be.

I am a kind policeman offering boundaries for safety.

An air controller coordinating a group.

A bird photographer waiting...waiting...waiting for that special sight of action.

A conductor, listening to every note in the score.

I am a child in pretend play, leaving this world for the imaginary.

In this room, I am not fully me.

I can forget.

I am free.

Then I met you, your questions making me remember.

You ask if my body has been ill like yours.

I pause.

Do I say I have blue eyes when I have chocolate brown and feel wrath of distrust, for you see otherwise?

But I didn't need to answer, did I?

You were told, by another, the reason for our delay in meeting.

You yawn and ask if I am tired too

We know that "tired" gives it no justice.

Buried behind the thick curtain of chiffon the world is hazy...distant.

You can't tear it, it will not rip, 
Keep trying and more will materialise.

Waiting is the only way for the chiffon blinds to temporarily part.

Yawn. Stop. Rest. Play. Yawn. Stop. Rest. Play.

We play in amongst the swirling dance of fatigue.

You inquire into what is engraved on my silver bracelet;

A tattoo of remembrance.

Soft pink was the colour you wanted for your medic alert charm.

Alarming red is what you got.

For me, the choice was simple silver, to camouflage the symbol.

Our bracelets jingle with the beating of drums.

Have you ever had steroids? You ask.

You tell me of the weight you have lost

now that you no longer need steroids and its ballooning effects.

I wonder if they rendered you an insomniac like me?

Watching the sunrise and listening to the first bird song while the rest sleep.

But we are all unique, side effects for some not others.

Trial being the only way to know.

Guinea pigs for poisons.

Can we play the drum?

Ahhhhhhh an easy one to answer.

Fresh scars from your surgery made for a one-handed drumming session.

I didn't remark on your right arm's holiday

and you didn't comment on my discoloured fingers.

The marks of the ill are perhaps unremarkable,

in the company of each other. 


With all these questions, are you searching for a bigger answer?
I'm afraid a comparison and my empathy won't give you one.
There is no reason why some are healthy, some are ill daily, and some die young.
It just is.
In this room, I keep my illness' entourage on a short leash,
So the pit-bull doesn't bite you.
For you, yours can run wild for the space of the hour.
Jumping, barking, running freely in its chaos.
I will throw it a bone to give it purpose and whistle it in when you need more order.
Should the leash I hold slip free, will my dog bark loudly and unsettle you?
I shudder to think.
I grasp the leash tighter and tighter,
For this room is for you to run free.

\subsection{Truth}

My feelings, described in the poetic letter above, prompted me to look to MT literature that was familiar to me, to better understand how it had become frightening to disclose in MT sessions. Why was I governing myself to hide? From reviewing the literature I had engaged with prior to and around the time of writing the above journal entry, two "truths" in particular seemed important and will be discussed.

- It is necessary for the therapist to determine boundaries

- Therapist disclosure/non-disclosure can cause harm

The first truth developed from the early psychoanalytic and medical approaches used in traditional MT. The second truth developed from both reading traditional and more contemporary discourses in MT. 
I was first interested by Rolvsjord's (2010) questions of, "Why are we so afraid of mutuality and closeness in the therapeutic relationship? Why are we not skeptical of the ethic implications of 'distance' in the therapeutic relationship?" (p.230). Distance is created in part by a lack of disclosure. The position of non-disclosure can be traced to MT's roots in psychotherapy and medical models where traditionally therapists were required to maintain distance with strict boundaries (Dileo, 2000; O'Grady \& McFerran, 2006). Self-disclosure infringing upon client-therapist boundaries and compromising professionalism, from this perspective, is thought to be an ethical concern that warrants risk management (Audet, 2011). My early training prepped me for this view. In documents of MT practice, client disclosures are far more prominent than therapist disclosures, which give the sense of disclosure being uni-directional.

The CoE in NZ states that therapists must, "establish and maintain professional boundaries in the client-therapist relationship" (p.1). Such documents position therapists as accountable for client safety and any harm resulting from boundary disclosure violations. However, different theoretical orientations would interpret that statement differently, as they allow different positioning's around disclosure boundaries. Traditional psychoanalysts are the least disclosive, while contemporary psychoanalysts disclose more. I became more interested in feminist- and resourceorientated approaches around 2011. Feminist therapists proactively disclose with the intention of reducing the power imbalance between therapist and client and to make the interchange more akin to being between two humans rather than patient and expert (Audet, 2011; O’Grady \& McFerran, 2006). Similarly, humanistic therapists disclose to convey their humanness and vulnerability so that the client is not made to feel inferior. In resource orientated MT, self-disclosure is used to support the collaborative relationship and authenticity of the relation (Rolvsjord, 2010).

Therefore, I became aware of the multiple truths that exist around whether it was best to self-disclose or not, which are fiercely debated and at times pitted against each other. For example, Rolvsjord (2010) says that "the need for a conceptualisation of the therapist's sharing must be seen in juxtaposition to the idea of distance as a necessary condition for a healthy therapeutic relationship" (p. 228). Self-disclosure can be viewed as both positive and negative, and arguments are made around how both disclosing and not disclosing can be harmful within different MT discourses. 
All approaches seem to have good intentions of protecting the client. In discourses that support self-disclosure it is done to protect the client from being objectified or taking a subordinate role. In the case of approaches that limit self-disclosure, it is to protect clients from role confusion, role reversal, burdening clients, and the therapist dominating sessions and making it about them. If I use such rationales (discourses both for and against) in this way, the decision becomes quite paternalistic. I note that the more contemporary discourses mentioned do support mutuality in practice in other ways. However, clients being seen as competent in sharing the management and creation of boundaries is often overshadowed in much disclosure literature, even though there can be different client preferences. From my experience, some prefer very flexible boundaries, while others do not.

From the literature I was familiar with at the time, it was the therapist that primarily made these decisions. Clients' thoughts, and ways of identifying client preferences of therapeutic boundaries, seemed to be lacking in the conversation. Therefore, whether or not to disclose was often at the therapist's discretion, as opposed to a joint decision-making and management process.

There was also a strong focus on what was best for the client and less acknowledgment of the potential impact on the therapist. Sometimes the therapist might not be ready to disclose (as was the case initially in the poetic letter above), sometimes they may be better off to disclose so that clients are more accommodating if they take time off to undergo treatment for example. In discussions around harm, this was less of a focus.

\subsection{Power}

I will discuss three potential power effects resulting from the "truths".

- Increased levels of individual accountability through the proliferation of $\mathrm{CoE}$, SoP and competency checking processes in NZ.

- Increased individual accountability/responsibility may create a strong sense of uncertainty (and perhaps fear) around disclosure decisions for the therapist.

- The potential for joint management of boundaries may be limited.

As responsibility is placed on the therapist to determine things like safe disclosure boundaries, an increase in accountability can ensue. Interestingly, since first 
becoming NZ registered, there has been an increase in measures of accountability. For example, the application to renew my practicing certificate moved from bi-yearly to yearly and supervision requirements increased. Within the broader context, the Health Practitioners Competency Assurance Act (HPCAA) was developed to protect the public from serious harm caused by health practitioners practicing below required standards. MT is not registered under this act because it does not meet the criteria set for the potential to do serious harm and is considered low-risk. Not being registered under this act can affect access to clients and pay disparity (Cornforth, 2006). Therefore, it may be beneficial in locating potential negative impacts of therapy within the individual therapist (including their disclosures) as describing MT in this way would associate it with professions that are perhaps taken more seriously under the act, resulting in increased levels of accountability.

With increased levels of accountability, and the underpinning of protecting clients and causing no harm in CoEs, the onus on the therapist to take a position for what's best for the client can feel like a weighty issue, contributing to the discomfort of potentially getting it wrong. As different discourses place harm either resulting from disclosure or non-disclosure, whether the therapist decides to disclose or not can be thought of as potentially harmful from the alternative position perspective. Therefore there is no "right" place to be. The power of the multiple truths and their resulting debate creates a constant tension in my thought processes. To illuminate what this is like, here are the type of thoughts that run through my head when I am in a situation like the one in the poetic letter above:

You asked a question. I am uncertain whether I should answer it. As Dewald (1994) suggests, would having an awareness of my illness make you filter your reactions to what you think I can tolerate? Are you downplaying your symptoms? Did I see your shoulders fall further between blinks? On the other hand sharing personal information can build rapport and enhance the therapeutic relationship (Ashmore \& Banks, 2002; Jones, 2012). However, I know there are some parts of your journey you must go alone. "Always to be accompanied, always to be understood would be intolerable" (Woolf, 1930 p. 39). I will save you from the "yes I know how that feels", for each person's illness is unique. But then again, might my 
own sharing make you feel less alone? The parts of me that I sometimes think of in terms of weakness or vulnerability might enhance the therapeutic process (Austin, 2002; Rolvsjord, 2010) or equally they may not. I have paused for too long and haven't responded. I'm scared to respond; it could be bad either way. I revert to my default response - to be quiet as much as possible.

The "truths" limit disclosure from being managed in a joint way by directly asking if the conversation is helpful for the client. The internal dialogue above can detract from listening and taking cues from the client as to whether they would find therapist disclosure helpful or not. The many questions the client asked in the poetic letter may have indicated that they would find some disclosure helpful. The discursive debate may detract from what the therapist might be comfortable with as the focus is primarily on the potential impact on the client instead of client and therapist.

\subsection{Ethical considerations}

The main ethical issue that the "truths" and power relations found in my practice create are that the partnership between therapist and client can be reduced to a more paternalistic relationship with harm located in individual therapists. Disclosure therefore becomes about doing something to someone else instead of a relational act between the therapist and client. Through a collaborative process, it may be determined where the boundaries feel comfortable for both therapist and client. This approach allows for flexibility to move between different levels of disclosure depending on client and therapist preferences. Competence is placed on being attuned to client and therapist preferences for boundaries, as opposed to whether or not disclosure or lack thereof it is harmful. In this way, disclosure is seen as a continuum where any position is valid and helpful if it is relationally negotiated. This may decentre the dilemma a therapist can have about what position to take.

Furthermore, as has been done in counselling in NZ, shifting the association of harm to unjust social contexts and lack of partnership/mutuality in $\mathrm{CoE}$ and SoP, instead of only locating harm within particular individuals (Cornforth, 2006), may also reduce some of the difficulty therapists may experience around disclosure. By doing this, we 
can recognise the harm that comes from wider social and cultural factors that may present in disclosing or not disclosing: the responsibility is shared.

This analysis raises an ethic of mutuality, where boundaries are co-managed and determined in partnership. The practice of music as communitas is helpful here, as roles and boundaries are more open to "processes of pragmatic negotiation within specific contexts and communities" (Aigen, 2014, p. 32). 


\section{Chapter 6}

\section{Falling in the Space Between: Oppositional Binaries}

"Playing dichotomies": A photograph of my piano.

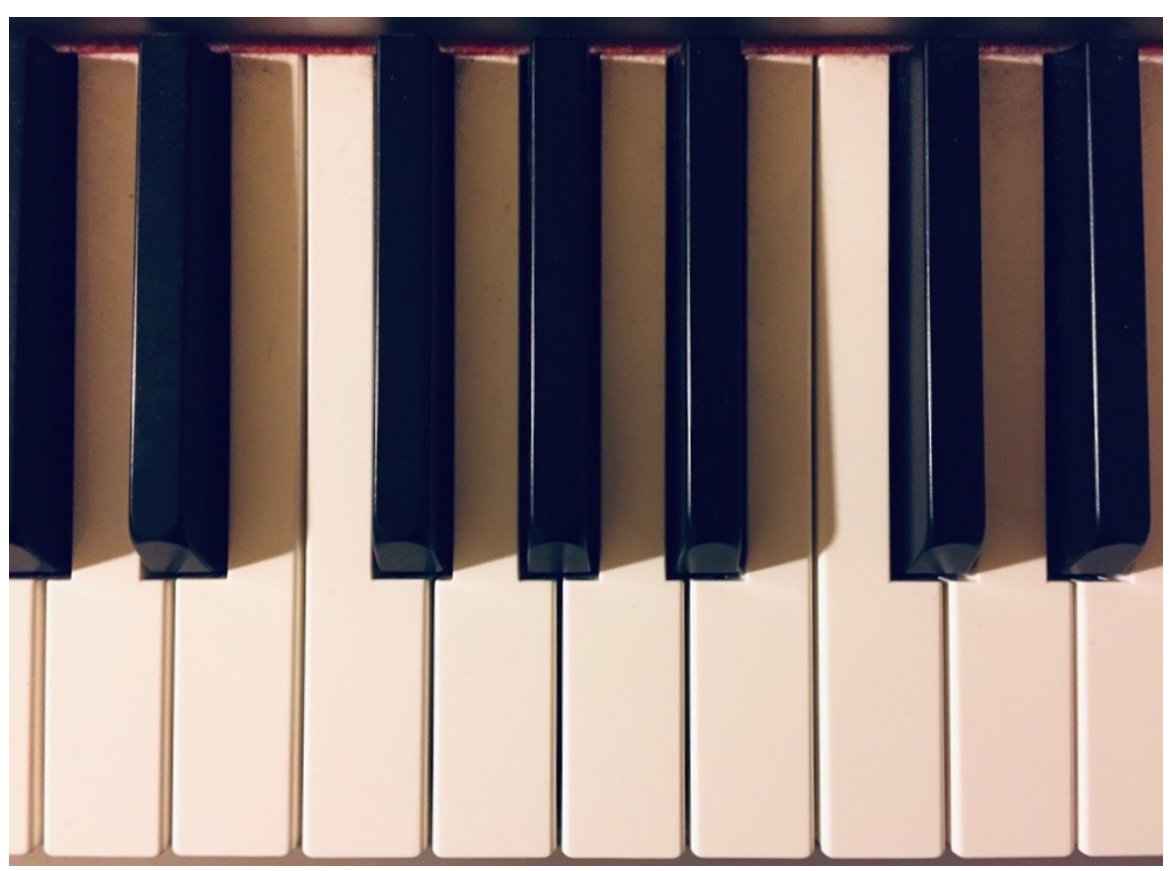

[Black and white piano keys with some shadows between them] 


\section{Introduction}

This chapter focuses on oppositional binaries. When looking through my journal data, I discovered a reoccurring concern around dichotomous thinking that seemed to limit my experience of illness/disability in some way: the dichotomies created a gap between language and my experience. When looking through these events/entries, I became aware of the space where dichotomies became unstable in my practice, and I started to recognise and value these more. This relates strongly to my research question, "How does the close examination of a practitioner's experience of illness/disability impact on their understandings and implementation of practice?"

As part of their ontological "truth", structuralist's believe that oppositional binaries are fundamental or foundational to the human mind (Davies \& Whitehouse, 1997). Western thinking is founded upon the logic of binary oppositions. Conversely, drawing attention to and deconstructing the thinking that underpins terminology has been a focus of poststructuralists (Thomas, 2007). Such thinkers have illuminated the arbitrary and inherent instability of oppositions. Derrida described how in oppositional binaries, one side dominates while the other is suppressed (one associated with positively and the other negatively), depending on the discourse (Skaife, 2013). They do not have equal opposing relationships of power (Cornforth, 2006). This privileging of one side is seen as natural and the way things are. The nondominant term becomes the marked term because it is different to and considered lesser than the dominant term (Cornforth, 2006). Therefore, dualismsnormal/abnormal, ill/healthy-can sustain the oppression of disabled people (Thomas, 2007): for example, the dominant ideology of ability is so much part of every thought, action, and judgment that it can be difficult to isolate, especially when literacy about disability is sparse (Siebers, 2013) because of its lesser term status. These binaries are set up through the boundaries made from specific socialisations, social expectations, and ideological constraints.

Yet there is no inherent logic to such oppositional binaries, as they are interdependent and indeed one cannot live without the other. A "both/and" orientation has therefore been proposed by poststructuralists and black feminists who have emphasised the importance of avoiding dualistic thinking (Feldman \& Tegart, 2003; C. Williams, 1999). Interestingly, like Skaife (2013), I have been conscious of the contradiction within binaries: they are simultaneously artificial and unhelpful, but often necessary. 
For this chapter's analysis, it was helpful to think in terms of Derrida's notion that one binary is given privilege over the other, and Foucault's tool of looking for what is not said. Both theorists and strategies helped me look beyond binaries and their hierarchies as adequate of finite meaning, so that a space could be created for absent voices/experiences in my work ${ }^{21}$. The intention was to move beyond the boundaries of the dichotomy. These dichotomies are so ingrained in western culture that they had become "common sense" in my music therapy (MT) practice. This chapter deconstructs this sense making.

From reviewing my journal entries and MT notes, four main interrelated dichotomies emerged $^{22}$ :

\section{- Therapist-client}

- Ill-healthy / disabled-abled

- Positive emotions-negative emotions

- Humans-Non-human

The term in bold highlights the term usually marked within my practice when dichotomous discourses were at play. I have done this to illustrate that these pairs were embedded hierarchically, and have put the lesser term in bold to increase visibility and to disrupt the default hierarchy. I recognise that other music therapists have thought and practiced differently.

These four dichotomies form the main headings for this chapter. The structure within each dichotomy follows my process of analysis:

1. The practices, conditions and discourses that sustained the "truth" of binaries in my practice in 2012 (truth)

${ }^{21}$ Derrida's idea of différance is also important to decreasing binary thinking. Différance plays on the fact that 'differer' in French means both to differ and to defer. Both meanings are always present and absent. Furthermore, because there are multiple meanings of a word, there can be no ultimate meaning, as it is constantly being defered. Linguistic meaning is therefore created not given. Meaning is created by the "play" of differences between words. By changing the usual spelling of différence to différance Derrida introduces a distinction that is only discernible in writing (the two spellings are pronounced identically).

${ }^{22}$ Other dichotomies were also found in my prior practice, such as biology (individual)-social (culture) and independent-dependent. These also support the disabled-able divide. However, including detailed analysis of all the dichotomies found in my practice was beyond the scope of this study. 
2. The power effects of the dichotomy (power)

3. Personal experiences that deconstructed/dismantled the dichotomous divide: Identification of non-binary moments (ethics).

4. Locating examples that deconstruct the dichotomous divide within session notes/practice: Examples where the structures themselves began to clash, overlap and dismantle themselves from within (ethics).

I note that the discourses discussed that constructed the dichotomies in my prior practice (under headings 1,2, and 3) are not the only discourses that other music therapists have engaged with. Rather, this is a study of my historical practice/experiences and how they came to be constructed. What I can do is be reflexive about myself. I do not intend to preclude other discourses. Some alternative discourses are also explored later in each section (under heading 4). Researching all that has been written about each dichotomy was beyond the scope of this thesis. 


\subsection{The practices, conditions and discourses that sustained the "truth" of the client-therapist dichotomy in my practice in 2012 (truth)}

Curious to know what my previous language in reports had been, I looked back at my notes from 2012. As my practice changed in response to my own experiences of illness/disability and $\mathrm{PhD}$ journey, going back to a prior time was important. Records were also easily accessible to me on my laptop. From reviewing these reports, three interrelated reocurring discursive practices emerged that separated clients ${ }^{23}$ and the therapist ${ }^{24}$ :

1) The therapist's actions and responses and the client's actions and responses were framed differently.

2) Clients' outcomes seemed to be portrayed as dependent on the therapist's actions (the interdependency of client and therapist actions lacked acknowledgement).

3) The client was positioned as in need of change (goals/focus areas) and the therapist positioned as an unchangeable agent.

These role binary sustaining practices support and were produced by discourses of expertise. The language used in reports pertaining to therapist and client differed. Depending on whether outcomes were desirable or not, student actions were framed either positively or negatively and drew on discourses of expertise, in which the therapist was positioned as assessor. Examples of negative descriptors of client's actions were: "behaviours", "control”, and "inflexibility". Positive descriptors for client's actions were: "participation", “initiations", “choosing”. In contrast, the expert therapist's actions were positioned positively with descriptors such as, "modelled", “encouraged", "challenged", "prompted" or "contained”.

\footnotetext{
${ }^{23}$ For consistency I use the term "client" in this section to refer to the person I was working with. However, due to working in education the term "client" is interchangeable with "student".

${ }^{24}$ I have used "therapist" instead of referring to myself in first person ("I") to show the clear distinctions made between client and therapist in these reports.
} 
Words used to describe client actions and therapist actions were different. How the therapist "controlled" a situation was not acknowledged in this way. Instead, words like "prompted" were used. When the therapist described using structured activities for "containment", it did not refer to the therapist's "inflexible' offering. If we reverse this for clients, they may have used "control" to remind others of their needs, and became "inflexible" as a self-strategy for containment. Therefore, they too could be "prompting" and "containing", but these terms were reserved for describing therapist's actions.

The therapist's actions were often noted in reports before a positive change was described in the client, giving the impression that they were the agent for the transformation instead of it being co-created. For example, one report stated, "I introduced structured activities and Terry became more settled and engaged in playing each instrument for longer periods of time". The client's actions that led to a change were predominantly framed as "choices" instead of "decisions". A choice is a response to an opportunity and alternatives, as opposed to a decision that can be generative without the existence of alternatives and may create alternatives from which to choose. This positioned client outcomes and processes as dependent on the therapist and the opportunities they create, rather than the interdependency of the interactions that produced them and the client decisions that led to them identifying their own set of alternatives. The positioning of the therapist as the "helper/giver" and the client as the "helped/receiver" contributes to this, as the therapist's actions "help" the client without due acknowledgement that clients also "help" therapists in generating outcomes.

Interestingly, while therapist actions were considered the agent for change, goals and focus areas were only assigned to the client. Clients were positioned as the person in need of growth in a particular area for the "success" of therapy to be assessed. Fairchild and Bibb (2016) noticed that academic writing privileges the challenges and negative aspects of people's lives in order to demonstrate the importance of the work of the therapist. Similarly, the notes hyper-focused on clients' areas of weakness for focus.

While the reports do indicate what changes the therapist made during the therapy process, such as moving from individual to group sessions, they do not address the ways the therapist personally/professionally developed within interactions to better meet the client. For example, did the therapist learn to wait for longer so that the 
client had more time to process and successfully respond? This was kept in the private space of supervision. The reports did not communicate that other people may also need to adapt their actions or develop in areas to improve outcomes for clients. The reports were perhaps written in this way as they were thought to be about the client. However, client outcomes and actions cannot be separated from the therapists: they are the result of an interactive, co-constructive relationship.

\subsection{The power effects of the client-therapist dichotomy (power)}

Six power effects of the either/or oppositional roles of the client-therapist binary are discussed:

- It creates a relationship where clients depend on the therapist's expertise

- Clients may lose their own "voice"

- Therapists are recognised for the success of the work, clients' contributions to the outcomes go unacknowledged

- It may impede the therapist's recognition of their own areas of development/learning or disclosure of disability

- Therapist deemed incompetent if they don't adhere the expert role

- Subjugation of other worldviews

It was helpful to consider the discursive power effects in my practice alongside discussions in Bruscia's (2014) book around "help" to guide the following discussion.

When outcomes are predominantly related to therapist actions, it creates a dynamic where clients are dependent on the therapist's expertise. For example when "help" includes things like, "giving them a voice", as stated in Bruscia (2014), it suggests that clients need to be "given" a voice by the therapist instead of "having" a voice they share/re-discover/discover in therapy that the therapist learns to hear it. When voice is "given", it begs the question of whether it is the client's voice or the therapist's. The power effect of the therapist's dominancy in the binary is that clients may lose their own sense of voice. To be "given" a voice, is to lose one's voice.

Within the discourse of expertise, therapists/doctors tend to be praised for therapeutic outcomes without acknowledgement also given to the client. The helper-helped binary that takes place within the client-therapist binary, supports this. Some therapists have objected to using "help" in MT definitions because it can easily become oppressive to the client (Bruscia, 2014). Bruscia (2014) makes the point that 
not all help is necessarily oppressive. While that may be, being thought of as in need of help, assistance, and reliant on others can be oppressive when it doesn't account for the ways the person also gives back, helps, and contributes in other ways to sessions or society. However, client and therapist are interdependent in helping. For example, remuneration for services is given to the therapist. They need this to enable their necessities of food and shelter. Therapists learn from clients, which helps them in their role (and possibly their own lives).

The analysis above indicates that the therapist can potentially dominate the client through using more positive terminology for their actions than that of their clients, for example. This differs to Bruscia's (2014) thought that "it's inauthentic to assume that there is a hierarchical power disparity between therapist and client and that the therapist and client have 'power over' one another" (p.294). He suggests that all clients have free will. However, looking closely at the reports, free will can be impeded within certain relations of power. Exercising free will may affect the quality of care received by being labelled as a "difficult patient/student" or "non-compliant" (this is demonstrated in the journal entries below).

Having strong distinctions between therapist and client creates an oppositional role rather than a "both/and" dynamic. In an oppositional framework, therapists may hide their disabilities to maintain strong distinctions between therapist and client, suppressing areas of their own need/development to reduce role confusion, reduce complexity, and maintain professionalism. A potential consequence for a therapist who does not adhere to the expert position is to be deemed less competent or lacking necessary abilities. When discourses of expertise purport the binary divide, it not only negatively impacts clients but therapists also.

I recognise that the maintenance of the therapist-client relationship disregards other worldviews. For example, an African principle is that human beings only realise themselves in moral relations with others (collectively, not individually) (Sue \& Sue, 2015). Collectivity is also important in Māori culture. The helpee and helper are not separated; they are bound, spiritually and ethically.

\subsection{Personal experiences that deconstructed/dismantled the dichotomous divide: Identification of non-binary moments (ethics)}

Below is a creative piece of writing based on a mixture of interactions I had with a number of specialists/doctors. Inbetween the dichotomous roles, there were moments 
where the boundary became dismantled and fell apart. The binary was deconstructed. The text positioned flush left (client) and flush right (therapist) pertain to the binary sides. The text in the middle deconstructs the binary. ${ }^{25}$

1 used to feel

like I did not have

anything to offer

them.

1 was a problem for

them to solve.

Medical voices were qualified to speak,

my bodily knowledge

lacked a certificate.

So, I said, "thank you".

At first my thank you was not returned.

I was to have nor-

epinephrine blood tests,

One while lying down,

one while standing up.

The nurse immediately started to withdraw the first sample.

“Stop" I said, "I have

only been lying down for

one minute. This test

requires that I lie down

25 I recognise that this is not ideal for some blind and low vision readers. I am happy to provide a copy of this thesis with the text flush left. 


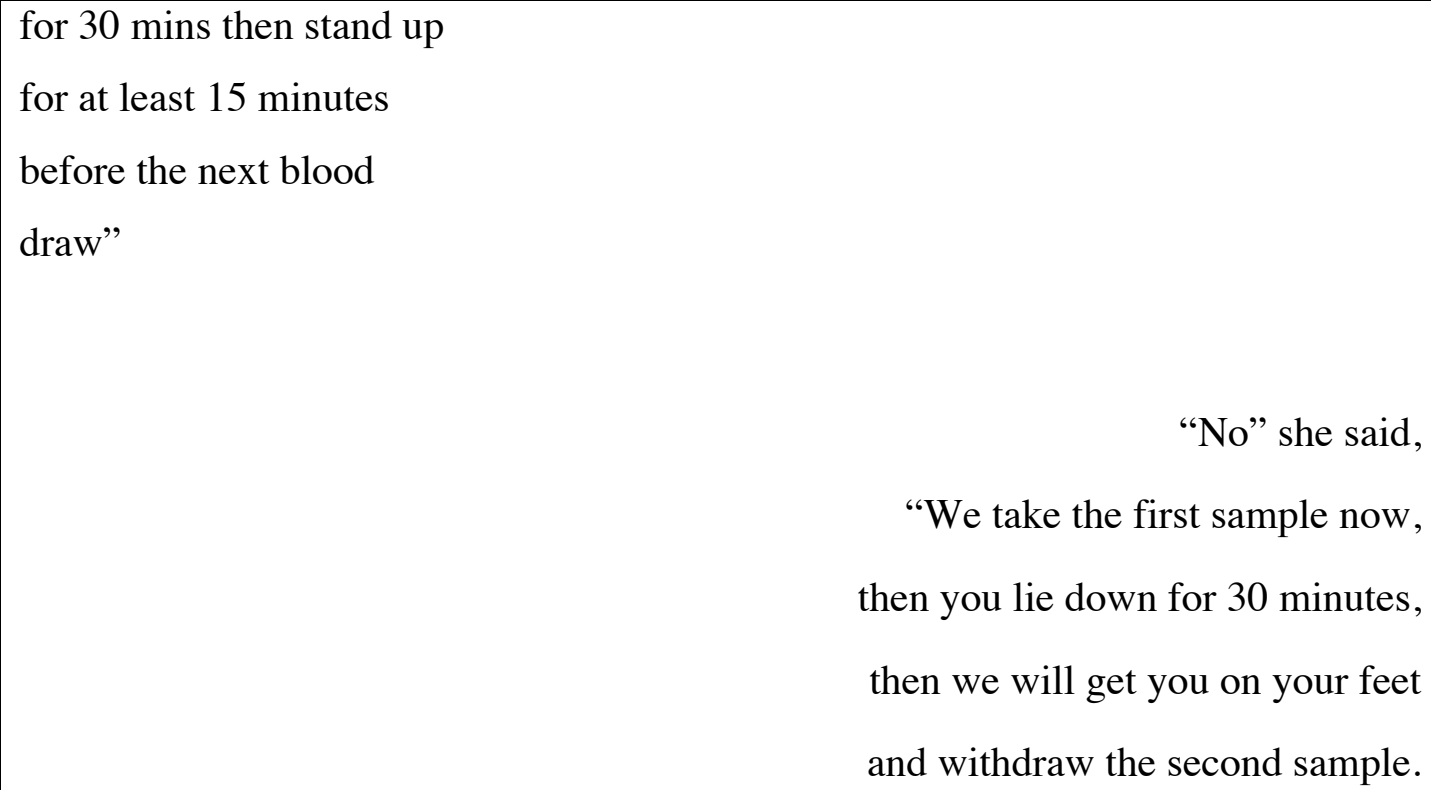

"We take the first sample now, then you lie down for 30 minutes, then we will get you on your feet and withdraw the second sample.

"I don't think that's right"

"Yes it is"

"It makes no sense"

"Yes it does"

"but..."

"Look I'm going to do this now"

"Please,

ask my specialist"

"She has already informed me"

1 comply.

My blood,

my time,

my afternoons income

was taken.

I emailed the specialist when I go home.

She replied:

“ Thanks for your email. That was not the correct procedure.

I will go up now to discuss with the nurse. 


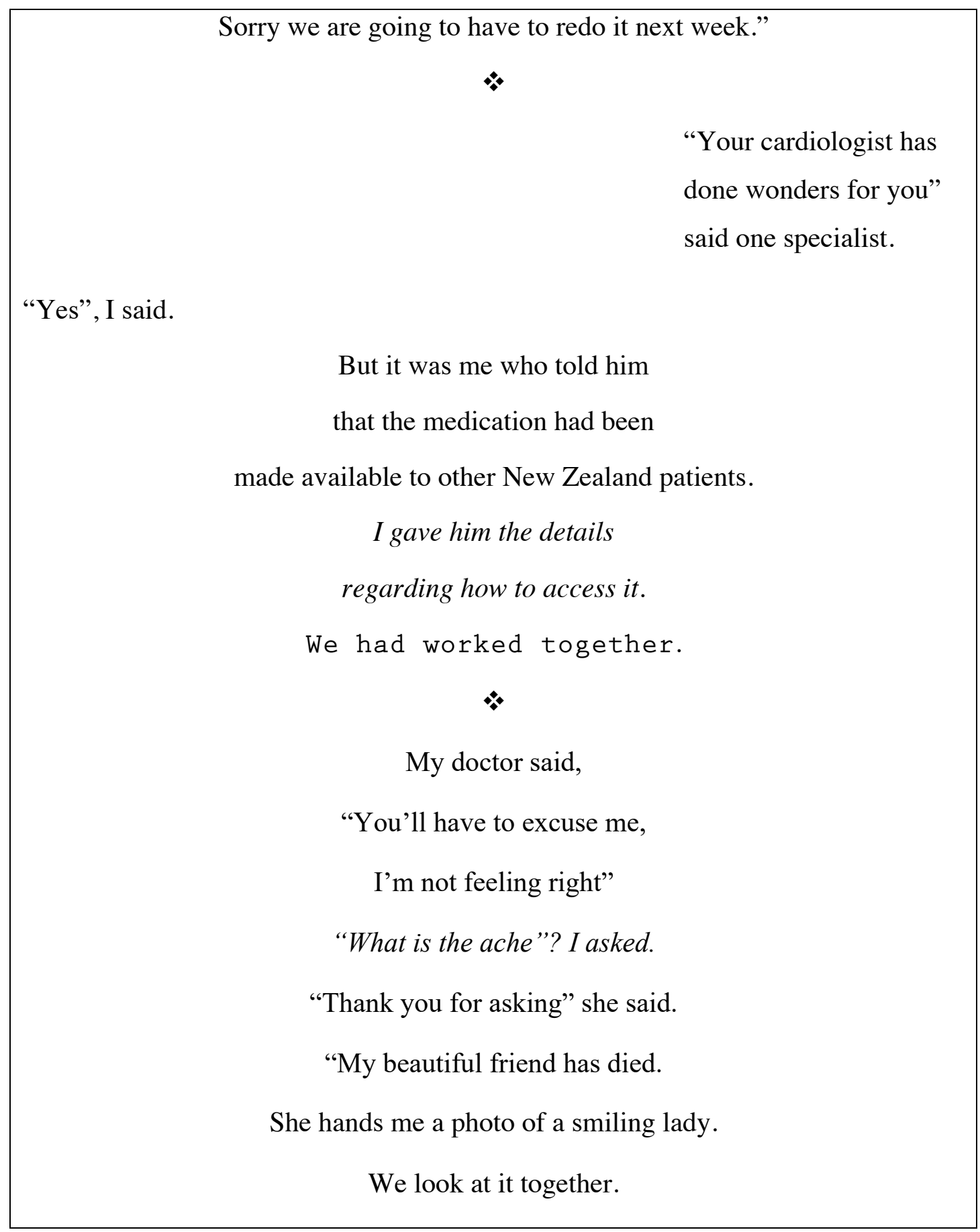

From this piece of writing, three points counter the dividing practices of expertise with discourses of mutuality.

1) Clients can be active agents/helpers in their own heath/wellbeing (their own inner therapist). This capacity can be minimised in discourses of expertise.

2) Clients can and do support or help their doctors/therapists both personally and professionally. Help is bi-directional.

3) Mutual recognition is integral in subverting the voicelessness and devaluing felt in the suppressed position of the binary. Clients too can be recognised for their contributions and their voices heard within mutual relationships. 


\title{
2.4 Locating examples that deconstruct the dichotomous divide within session notes/practice (ethics)
}

This section will discuss MT examples that existed beyond dichotomous boundaries and understandings associated with discourses of expertise in an ethic of seeing the marked term (client) as inherently valuable and its interdependence in the therapy process. Counter discourses that reduce the binary divide in the literature are briefly mentioned below as they helped me to locate the examples alongside my own experience. The counter discourse of mutuality is increasingly discussed in MT literature, such as in Community Music Therapy (CoMT) and Resource Orientated Music Therapy (ROMT) (Aigen, 2014; Rickson \& McFerran, 2014; Rolvsjord, 2010, 2014). Mutuality can be supported by thinking of MT as taking place in an aesthetic interplay rather than an hierarchical relationship where the patient is sick and the therapist is well (Kenny, 2006). Rolvsjord (2015) highlights the bidirectional nature of the therapeutic relationship. When mutuality is present, roles and boundaries are open to negotiation and the focus shifts to include what the client does in the experience to promote outcomes.

The Māori discursive idea of "Ako"-sometimes teacher, sometimes learner-and the discourse of "the wounded healer", were used in identifying the following experiences. The relationship within these discourses moves to one of interdependency. The writing below is a collection of interactions from my MT practice that draw on the discourse of mutuality.

\section{I have been offered food.}

It is their way of nourishing me.

I ate fish and chips with one family as we talked about the child's session.

Another gave me her freshly baked sourdough bread.

Once, a gluten free chocolate cake was placed in my hands:

Giving an energy burst for that last hour of work.

\author{
$*$ \\ "Thank you" \\ They said. \\ "Thank you"
}


I said back.

We both grew together.

I took two loads of instruments to my car,

I walked across the playground.

It was the stairs that did me in: Passed out on the office room floor.

As I came to, I heard a little voice while we waited for the ambulance.

"Ohhh, Carolyn, what happened, are you sick", said one of my students.

She knelt beside me and placed her warm hand on my shoulder:

An action she usually receives.

"I am ok, I am just like the Bunny in our Bunny song" I said.

In the song, Mr. Bunny starts bouncing once he is better.

"Oh I'm sorry" she replied, as she started to play with the things in the office.

I supported her by explaining in a way she could understand while she offered her support.

We were both helper and helped.

As for the other student who saw me on the office room floor, we didn’t speak until his next session.

It was he who was asking the questions:

"You collapsed eh?"

"Did you run around too much?"

I was now in the position of answering.

A different way for each of us to be in the relationship.

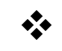

It was the students who thought of making a music video.

It was a parent who suggested that her child needed more time to get to know me before we tried engaging musically again.

It was a teacher who suggested a music resource. 
It was not always I, the music therapist.

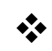

2016 MT reports note what I had learnt in the process: noting actions that may not have been helpful. This differed to the 2012 reports.

For example, in one 2016 report it states:

"To begin with, Louisa preferred shorter sessions. She would ask to go to the toilet after about 10 minutes. I initially interpreted this as a genuine need for the toilet.

However, I realised after two sessions that she was actually communicating a needed break or to finish. Short breaks/spaces between interactions were henceforth incorporated into sessions." 


\subsection{The practices, conditions and discourses that sustained the "truth" of the binaries in my practice in 2012 (truth).}

Like the previous section, I have looked back at my MT notes from 2012 in conjunction with considering the general context of my practice. I will discuss three discursive practices that supported dichotomous thinking regarding disability and illness in my previous work.

- The disabled or ill aspects relating to the person are predominant focus areas of sessions. This supports and relies on a problem-orientated deficit discourse.

- Dichotomies were produced within and between disability and illness, supporting the idea that disability and illness can be separated. This is produced by both social model and medical model discourses of disability.

- Disability and illness were presented as stable across contexts/environments. The discourse of the medical model of disability produces and relies on this.

In reports, identified areas of focus were predominantly problem orientated and/or related to the person's impairment. For example, "Peter will successfully take turns with a wider variety of peers". Focusing on supporting aspects of the person that were well/able did not sit alongside these goals. Goals were not open to the possibility of ability within the identified disability. While the well and able aspects of the person were mentioned in reports, they were mentioned disproportionately less and minimised by their lack of appearance in the indicated focus areas for sessions. In this way, the problem-oriented deficit discourse aligned with a pathogenic orientation that understands health as an either/or state (Bruscia, 2014).

It is interesting that the dominant terms of the binary-health and ability-are less visible in the areas of focus, and the suppressed terms of the binaries are the focus. A reason for this may be, as Fairchild and Bibb (2016) point out, that to obtain funding the need for the service is emphasised. Illness/disability within a deficit discourse is an abnormal, undesirable state of being that requires focus to lessen the undesirable implications of these states. Therefore, the divide between health/ability- 
illness/disability is generated in part due to dichotomous thinking of health/ability being good and desirable, and illness/disability being bad and less desirable. Nowhere in my reports does it state how the person's disability or illness has enhanced an aspect of the client's being.

Even within contemporary MT discourses that focus on wellness rather than deficit, the generativity of incapacities, limitations or disabilities is not adequately acknowledged. Here, capacities rather than limitations are the focus of attention, supporting the other side of the dis/ability divide. Growth directly from the limitations of impairment is often lost or not a focus. However, studies of disabled musicians demonstrate how their limitations resulted in prized originality because of, not despite, limitations (McKay, 2009). Examples of this include Joni Mitchell's open tuning guitar technique (due to polio) and Carl Perkins's adapted position for jazz piano created exciting new sounds for their audiences (McKay, 2009). This connects with Nachmanovitch's (1991) idea that "sometimes we damn the limits, but without them the art is not be possible. They provide us with something to work with and against" (pp.80-81).

The reports analysed don't seem to align with a pathogenic orientation when it comes to diagnosis, as diagnoses weren't mentioned. Within the broader context of my work, however, categories and diagnoses do have a strong presence in how disabled students are described. There are even categories within a disability/impairment, for example, high functioning and low functioning. Such categories are present in MT research (Lim, 2010a). Strong distinctions are also made between health and disability due to different funding pathways available to the person seeking therapy, such as students referred from health services described as having "health needs", while those from educational services have disabilities or "learning support needs". The differing discourses around illness and disability create a dichotomy between them. This affected the language I used in the reports. Outcomes for disabled students were associated with new abilities or skills, while outcomes for those who were ill were associated with increases in wellbeing. Illnesses within disability and vice versa (the ill-disabled) were not presented as such (one aspect was chosen over the other). This demonstrates that binaries within binaries, and binaries between binaries, exist. Contributing to the idea that there are divisions between them all.

The separation of illness and disability can be seen within both the social model and medical model of disability. Within the field of disability studies the prevailing 
position is that disability and illness are two different phenomena (Souza, 2014). The NZ Disability Strategy, which draws on the social model, seeks to "ensure disability services do not perpetuate the myth that disabled people are ill" (Ministry of Health, 2001, p. 21). This affects the unhealthy disabled (those living with chronic illness). There are connections here with the body-mind divide, which raises similar issues.

Within medical model discourse, the illness/disability a person is experiencing is presented as stable across contexts and environments. For example, classroom observations were often used to validate the need for MT. However, if the person presented differently in MT, such as their disability was not/less present, they were still considered as a disabled person in need of therapy based on classroom observations. The reports didn't take into account that illness/disability is an unstable state that can be present in some contexts and not in others. They change in relation to different situations. When illness/disability is understood as a fixed state of the individual and contextual constructions of disability are suppressed, dichotomous thinking is supported.

\subsection{The power effects of the dichotomy (power)}

The main power effect is that binaries are exclusionary and create exceptionalism. This contributes to the misrepresentation of disabled people. Discussed examples include:

- The ill-disabled can be excluded from either category.

- The challenges of "high functioning" individuals may be minimised and the ability of "low functioning" individuals limited.

- The generativity of limitations and incapacities to bring about some abilities because of, not despite disability, are often not represented.

- To maintain the binary, Disabled people are often not represented as living everyday lives. They are othered in the form of "disabled heroes" or the "helpless".

- The psychic wellbeing of "normal" non-disabled people rests on the binary divide to the detriment of the psychic wellbeing of disabled people.

- It leads to a limited understanding of disability.

- It results in a lack of ways to language the experience of being disabled-abled. 
If illness/disability is focused on for direction, a large part of the persons whole experience is missing and therefore denied (Lindsey, 1996). It excludes the possibility of high levels of health in the presence of chronic illness and high levels of ability in the presence of disability (Lindsey, 1996). Going further, the illness-disability divide excludes the experience of the ill-disabled. It makes little sense to separate disability from illness, when disability is framed as being produced by interactions between that which is biological/physical with that which is sociocultural, historical, linguistic (Souza, 2014). Binaries within the disabled-able binary contribute to the misrepresentation. For example, the binary of high and low functioning can minimise the challenges of people classified as high functioning and limit the view of the person's ability when they are labeled as low functioning (Prizant, 2015). All of these contribute to an identity politics that rest on the normal-abnormal divide.

Another power effect is that, as the marked terms in the dichotomy, illness/disability are positioned as only bad and some sort of limit without possibility. However, positive and negative aspects co-exist with possibilities generated from limits. The poet Robin Morgan said, "I do not feel diminished by Parkinson's; I feel distilled by it, and I actually very much like the woman I'm distilling into" (R. Morgan, 2015). The fact that illness/disability can be a positive contributor to life and a catalyst for psychological growth is often overlooked (Kielhofner, 2005; Vash, 1981) within deficit discourses. For example, Jazz musician Horace Parlan and saxophonist David Sanborn took up their instruments in childhood as part of their physiotherapy for polio. It is "arguable that a successful music career came about because rather than in spite of the childhood disease" (McKay, 2009, p. 348), as this contributed to their unique playing styles and sound.

The two predominant societal narratives of disabled people, the vulnerable and helpless or the disabled hero, both rely on a negative view of disability that perceives achievements as being despite the disability. If disability/illness were understood as having both good and bad aspects, it would be more likely that the media would portray disabled/ill people as living everyday lives. The binary created through negative associations positions the disabled as other to sustain the psychic wellbeing and comfort of those who can obtain that they are normal (with good lives) by contrast.

Another power effect is that, if illness/disability is understood as something that the person either has or doesn't have, context and culture may not be taken into account 
in the therapy process. Health is a relational phenomenon that varies across contexts. There are cultural differences. Disabilities in one context, such as UK, may not be disabilities in Sarawak (Ling, 2012). Or how the person is disabled in the classroom, may not be present in the MT room. Therefore, the terms "disability", "illness" and "health" are floating signifiers. A floating signifier is a word that has no agreed upon meaning, it means different things to different people. Dichotomies make it appear that the meaning of disability is clear and simple: meaning the inability to do something. However, in disability and social research, there is no consensus on what constitutes disability (Mitra, 2006). No single model can totally explain it (Pfeiffer, 2001). This lack of a clear definition destabilises the boundaries of the binaries. When inconsistent definitions are hidden, simplistic understandings of disability take hold (for example, maintaining a person's disability status across contexts).

It is difficult to get around using the words "disabled" and "able-bodied" and their associated power effects because they are the most available and accepted in communicating with wider audiences. For example, LaCom and Reed (2014) mentioned that they used the terms "able-bodied" and "disabled" with reservations due to their role in shaping binary understandings of embodiment. However, they proceeded to use the terms, as they were the most accessible short-hand available to them. A final power effect, therefore, is that other ways of languaging the experience of the disabled-able are largely missing.

\subsection{Personal experiences that deconstructed/dismantled the dichotomous divide: Identification of non-binary moments (ethics)}

The writing below is a patchwork of journal entries. To escape binary thinking that failed to capture my experience, I needed to create my own language at times (newly created words and definitions are in Imprint MT Shadow font).

\section{There is constant change.}

My identity, my body, the places I go, move across borders so often, that I can't locate them.

At 8:30am I eat breakfast then walk to the mailbox 
before heading to my

first session.

At 11.00am I'm sucking ice chips and hugging walls as I walk before star fishing on the couch to sleep.

At 4pm, I go to the

library and read on the

couches.

At 5pm, I meet my husband at an art gallery then abruptly leave: there were no seats.

Between the worlds of the ill/disabled and well/abled,

Wordless,

I travel in search of poetics. Creating words for these new states of being.

\section{'Functionoflux'}

$\mathrm{n}$. The state where your function in the world is always changing and moving. The state of wanting to play with sand, to sculpt it, and preserve it when it's beautifully balanced, but never can for the wind. You can only but watch as it moves to anyplace at any time. The boundaries of the beach don't contain it, as you scrape the sand from your ear.

Sometimes I am positioned as disabled to not be disabled.

In the terminal, the announcer asked, 
"Could passengers with disabilities and those travelling with young children please proceed to board the plane".

If I board the plane

before others, I am not disabled.

I will not have to stand

in the aisle queue fighting for consciousness,

Legs dragging.

To board the plane early to not experience disability

I become disabled.

\section{'Multiclone'}

$\mathrm{n}$. The awareness that you can be in multiple places at one time, while not knowing where you fit best. Like putting your home into boxes with the wrong label, your system of knowing where to put things is messed up. The toothbrushes end up in the laundry box. Flattening the boxes, you realise that the toothbrush is a good cleaning utensil. You put one in the laundry and one in the bathroom.

"Do you let it hold you back?"

The reporter asked the disabled man.

I laugh.

It tugs at my hoodie when casually out,

It tugs at my slippery heel when naked in the shower,

It tugs at the collar of my work shirt and my PJ's when I climb into bed.

There is no choice to not "let it" hold me back. 
The reporter failed to see that being held back by his impairment is what simultaneously propelled him forward.

Now that I can no longer work full time, I can study again.

Now that my body can't travel far, my mind travels.

Now that I can't garden, the flowers look brighter.

Now that I can't sustain walking, fitness is regularly scheduled.

Illness and disability were never a limit without possibility.

Sometimes I grow because of, not despite, the limits.

\section{'Heldangrow'.}

n. The understanding that, like tomato plants, we can grow taller being tied up.

Key points emerged from the above writings that countered the identified discourses that constructed binary thinking:

- We can relate to illness-health and disability-ability on many different levels and ways.

- Illness/disability is not a stable state. It is fluid and can change moment to moment. Accordingly, one is not either disabled or able-bodied/minded, but instead temporarily disabled and temporarily able-bodied-minded.

- Health/ability and illness/disability are not dichotomous. As an organic unity, the body's sensibilities of health and illness cannot be divided into categories; instead there is a sense of wholeness where the experience of health and illness coexist (Lindsey, 1996). A high level of health does not mitigate the level of illness. To express the diversity of illness experiences, the concept of health within illness was developed in the 1990's (Carel, 2013). Recently, disability scholars have proposed an inclusive mode of embodiment that encompasses human diversity that subverts the binary division that separated ability from disability (Howe \& Jensen-Moulton, 2016).

- Health only becomes meaningful when it is confronted with illness (Lindsey, 1996). 
The new words and definitions I developed to describe my experiences can be encapsulated within an identity of what I call "ability fluid"26. "Ability fluid" allows one to identify as a dynamic mix of disabled and abled, destabilising the boundaries between these categories through acknowledging the changeability, fluidity, and inseparableness of each state.

\subsection{Locating examples that deconstruct the dichotomous divide within session notes/practice (ethics).}

MT examples are discussed that existed beyond dichotomous boundaries and understandings in an ethic of seeing the marked term as inherently valuable and its interdependence in the therapy process. Some music therapists have outlined a practice that addresses the issue that clients cannot be categorised and labeled according to disability. Kenny (2006) outlines issues that can be supported in practice, such as, "how do we allow ourselves to experience beauty and fill our aesthetic needs?" and "Can we recognise and accept patterns which connect us", which do not rely on disability categories (p. 5). This allows for the possibility of health within illness, regardless of the physiological condition; aligning with studies that show how individual variation in well-being is not accounted for by disease condition (Carel, 2013).

I have been seeing Pippa for many years.

She has grown and I have too; our haircuts have been one of the many changes.

Her disability is funded as 'very high needs' at school.

But when she walks in the room the light is brighter, and the smiles of others become richer.

"She is tonic"

Her godmother told me. I agreed.

She cannot talk.

But she is rich in smiles and love.

When her Daddy returns from a work trip, she will not leave his side.

26 There were parallels with the term "gender fluid". Both gender fluid and ability fluid describe a both/and orientation. There seems a wide range of terms and ways of identifying in gender studies compared with disability studies. I, therefore, drew on gender studies in labelling an "ability fluid" identity. 
She laughs when I say "uh oh".

\section{'Multiclone'}

Due to her disability, she likes to make sure things are predictable and as they should be: She helpfully reminds me where things go and gets my guitar pick for me if I forget.

Due to her language disability, her music is wordless, yet we create understanding all the same. Her music was uninhibited by words.

\section{'Heldangrow'}

Pippa's particular creations were made from her limits: open string symphonies of beautiful simplicity and vowel sound operas.

Between our musical improvisations her hand slapped hard against her chest signing for 'more'. The gesture powerfully communicates, we play again.

\section{'Functionoflux'}

At the begging of the session,

She made her own choices.

A seizure then unmade them.

I chose to sing her a gentle song.

Pippa's goals were reframed.

I wrote: "Areas and possibilities explored in 2015"

1) Social understanding

2) Self-expression, creativity

This reframing allowed for disability/ability and/or illness/health to co-exist and to both be recognised in session outcomes. 


\subsection{The practices, conditions and discourses that sustained the "truth" of the negative-positive emotion binary in my practice in 2012 (truth)}

Discourses of positivity had produced a positive bias and dichotomy in my prior practice. Whilst the holding of negative emotions was evidenced in the 2012 MT reports, this space was thought of as creating opportunities to express and release negative emotions (the undesired state) without acknowledgment of the generative/functional possibilities of negative emotions. Positive emotions were always portrayed positively. The reduction of negative emotions was seen as a positive outcome. Therefore, supporting the binary divide that "positive" emotions are desirable resources and "negative" emotions are not. Positive emotions were paired with joy, happiness, excitement, and anticipation. Whereas anger, unhappiness, anxiety, fear, loss, denial, apathy were positioned as negative emotions. These correlate with dominant western labeling of "positive" and "negative" emotions.

To understand how my practice was more positively-biased and dichotomous, I will discuss discourses of positivity in MT that I had engaged with, as well as the dominance of positivity in my social context $\mathrm{t}^{27}$. I had become increasingly interested in ROMT around 2012. ROMT is framed with the labeling of "positive" and "negative" emotions. Although it doesn't ignore "negative" emotions, it also privileges positive emotions. One of its main focuses is to "foster positive emotions" (Rolvsjord, 2010, p. 54). This approach has been based on positive psychology. The field of positive psychology focuses on the development of positive experience, such as well-being, happiness, \& hope (Rolvsjord, 2010). Such an approach doesn't intend to devalue the role of and attentiveness to negative emotions, it was developed to counteract the negative bias in psychotherapy.

\footnotetext{
${ }^{27}$ I note here that different discourses may produce a negative bias rather than a positive bias in a therapist's practice. For example, Bodner and Polansky (2016) discuss how those with a strong psychodynamic orientation may perceive the session to be more meaningful and authentic when negative emotions are expressed. They also found that the perception of negative emotions being more meaningful to therapy was strongly related to a low level of professional experience. Therefore, the theoretical orientation and experience of the therapist influence how this binary might be enacted in the work.
} 
Whilst the positive psychology movement was a corrective supplement to mainstream psychology, not an alternative (Rolvsjord, 2010), it seems that it has influenced societal discourses of pop psychology to an unhelpful level for disabled people. Cando optimism has become a Western cultural myth. As a child I was often read the book The Little Engine That Could, learning the message that positive thinking can overcome obstacles. Stories of disabled people overcoming through positivity have become moral lessons for the reassurance of the healthy/non-disabled (D. Wall, 2005).

People with illness/disabilities are constantly encouraged to have a positive mind-set and a can-do attitude. This is thought to decrease their illness/disability and/or improve their lives. The television programme showcasing the lives of disabled New Zealanders is called Attitude. At Victoria University of Wellington, discourses of positivity are prevalent for students with disabilities, repeating the message that the main thing is attitude and difficulties can be conquered by the right frame of mind. One of the criteria for assessing students for a disability access award at Victoria University of Wellington, 2016, is having "a positive attitude". Interestingly, the awards/scholarships I applied for, which didn't relate to disability, did not have this criteria stated. In the information pack I received from disability services in 2014 (which overall I have found to be a helpful university service) was a resource sheet about building resilience. On this it encouraged disabled students to have an optimistic outlook. This contributes to the expectation that "disabled people are expected to keep their happy smiles" (Cameron, 2009, p. 388). In broader contexts of social media, positive discourses continue to reinforce this message with popular memes like the one below:

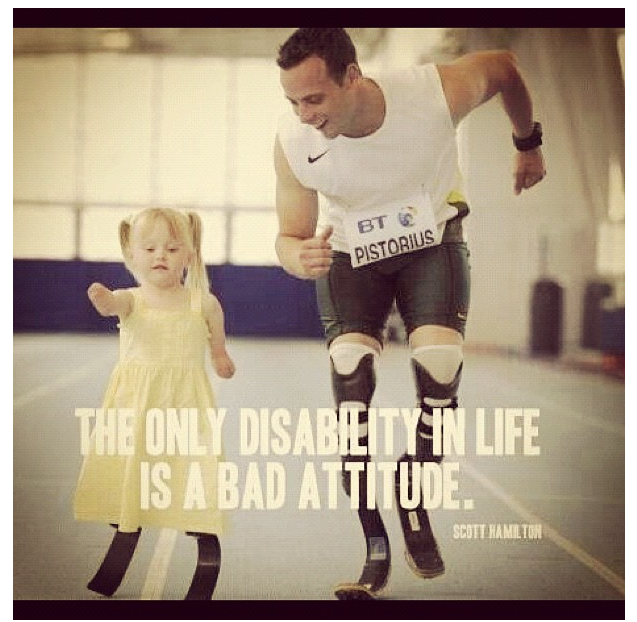


[Image of a young girl and man running with prosthetic blades. The caption says, "The only disability in life is a bad attitude]

The cultural myth of positivity overcoming disability is often maintained by silencing the messiness and range of emotions with popular news statements like, "they never complained", praising disabled people for their positivity. Looking back at my notes, I can also see this. A goal mentioned in one report was to reduce "I can't statements".

Privileging "positive" emotions/thoughts has played a role in disability studies and activism. The absence of upsetting or "bad" emotions in this field has been noted by some authors (Donaldson \& Prendergast, 2011). A NZ example of disability activism silencing negative emotions can be seen in an activist's blog who told other members of the disabled community to "harden up" and not to portray themselves as "traumatically wounded". He called for sharing stories of "way to go" instead of "woe" (Patston, 2015). An apology followed, however, it demonstrates the prevalence of privileging positive emotions and silencing negative emotions. In order for disability to not be seen as the lesser binary term, some activists have dissociated it from negative emotions because they too have the lesser term status.

\subsection{The power effects of the dichotomy (power)}

The power effects of this dichotomy and the privileging of positive emotions are:

- Negative emotions become unwelcomed, fraught with stigma and silenced. Consequently, disabled people can be denied the full range of emotions.

- The generative and functional uses of "negative" emotions in a person's experience are limited, whilst the potential of positive emotions to impede can go unrecognised: positive-negative emotions and negative-positive emotions become invisible.

- Illness/disability can be attributed to an individual's lack of a positive mindset, which denies cultural and social disablement.

I preface this discussion with the point that I am not suggesting that getting stuck in an emotion that causes distress isn't problematic; more that all emotions can be valuable have the potential to be both positive and negative. For example, anger can propel one to action and denial can give one a break from difficult realities.

The idea that prevails in western society that happiness is a default position and that everything we do is in pursuit of it, has created a fear of sadness (Mackay, 2013). 
Whilst it is thought that we grow through pain we are simultaneously given the message to "move on", and "cheer up!". This can actually create a need for therapy, as talking about negative emotions becomes unwelcome and silenced in social interactions where these discourses pervade. People often don't like hearing the raw sound of pain (D. Wall, 2005). Even in therapy, positive emotions can be strived for when these are privileged. Experiencing or voicing a full range of emotions, in which disappointment, frustration, and failure are a part, can become limited in some situations.

Disabled people in particular are impacted by such discourses as they are often used as examples of the power of positivity. Inspirational stories of disabled people tell us that we should be fighting the good fight, and to always have hope, and so forth. When narratives of overcoming by remaining positive dominate, individuals can be blamed for creating or not overcoming their disability through a lack of positive attitude. Thus, voicing difficult emotions becomes fraught with stigma, the fact that some things can't be overcome by a positive attitude is denied, and the potential harm from a positive mindset is not considered along with its helpful possibilities. For example, thinking you can do a task that pushes you past physical limits in chronic illness, can lead to a flare in symptoms and a decrease in function. It can negatively impact one's health.

Disabled people are denied a full range of emotions when success is framed by positive emotions. Prominent news statements like they "never complained" praises the silencing of negative aspects and shuts down the expression of negative emotions. This leads to thin descriptions of feelings and simplified understandings. Hyper focusing on positivity, and not being offended by your illness, conforms to the expectations of the healthy to be the good patient. Being praised for your behaviour confirms you status as conformist (Carel, 2013). Non-conformists go unpraised and negative aspects become socially difficult to speak of. Social benefits are gained by hiding realities from the non-disabled and well (Carel, 2013). When topics other than the difficulties of illness are discussed, there is the social reward of being seen as brave, graceful, a good sport, the one coping well (Carel, 2013). Conforming to these expectations and societal demands perpetuates the "truth" that disabled people can overcome with positivity. What is tragic about illness/disability is this silence (Carel, 2013). Disabled musicians have referenced this silencing in their song lyrics. Folk musician Ian Stanton in "Remember Douglas Bader", wrote (Cameron, 2009): 
'Cos that's what my doctor said to do

Overcome those negative feelings...

And I will smile

And nod and smile

And I'll be happy all the while

You might think I'm a hero too...

The idea that positive emotions/mindset can overcome disability ignores the social aspects of disability. The late Stella Young, Australian disability activist, noted this in her Ted Talk. She said that the statement that "the only disability in life is a bad attitude" is incorrect because, "no amount of smiling at a flight of stairs has ever made it turn into a ramp. No amount of standing in the middle of a bookshelf and radiating a positive attitude is going to turn all those books into braille” (S. Young, 2014).

\subsection{Personal experiences that deconstructed/dismantled the dichotomous divide: Identification of non-binary moments (ethics)}

A mixture of short extracts from my journal is presented below. There were moments where the boundaries between "positive" and "negative" emotions/thoughts dismantled themselves and fell apart. The text positioned flush left ("negative" emotions) and flush right (positive emotions) pertain to the binary sides. The text in the middle deconstructs the binary.

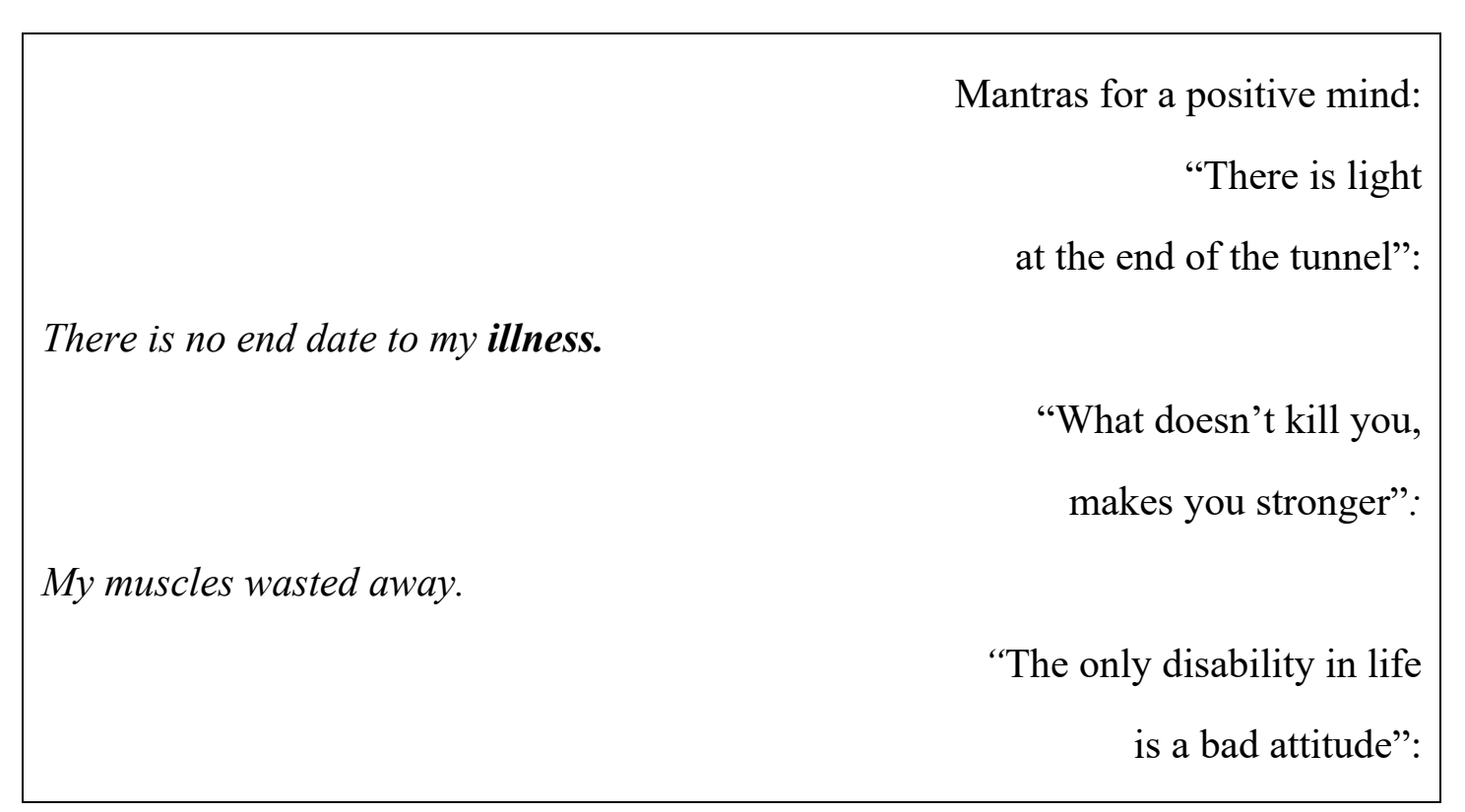


"I think I can" and fainted.

"If life gives you lemons, make lemonade":

My stomach aches with lemons.

It is what you believe this means

for my life that makes

honest disclosure uncomfortable.

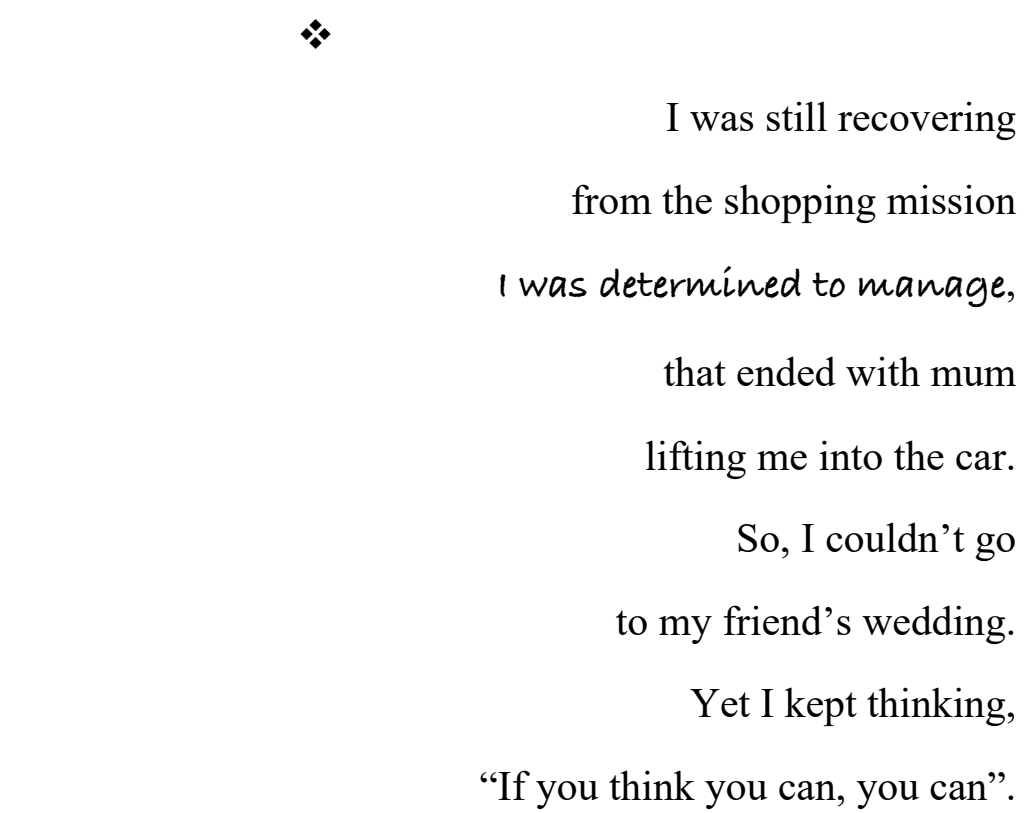

A mantra that has hurt me physically

and mentally.

I begin to mend,

with the words,

"I can't".

Anger brought the action

of better medical treatment.

Apathy brought a much-needed 
"There was loss,

and there was joy, and there was a state in which both of them were one"

(McGrath-Smith, 2011, p. 286).

I thought the petrol tank fueling

my hope was on empty.

That I had bled it dry with too many

frowns and turned down lips.

I had resigned myself to the

grunting and growling of a car on empty.

It was OK, I would drive slow.

But as I opened the envelope hope grew and filled once more.

Tears fell while reading.

"Complicated...rare...her conditions

are difficult to manage together...

sorry there are no easy answers".

The long list of medications tried

with no meaningful success and

the empty list of other medications

to try was disheartening.

With that, hope was emptied once more.

Hope helps and hope hurts.

We discussed nail polish colours between the echocardiogram, CT, 
x-rays and bloods drawn.

"Be careful inserting the cannula" the consultant told the nurse, "She is getting married in a couple of days and still has bruises from her last admission, let's not add to them". Even as the needles were inserted, we all held hope that all it would take was a change in medications.

As my sister arrived back from her manicure

bad news was broken.

Her pretty nails were out of place

as we heard the cry of hope breaking,

so she hid them.

The shattered glass of our hopes,

formed a puzzle for new ones to be found.

As we flew in the air ambulance to the bigger hospital, we hoped we would get and find home soon.

People crossed fingers, set dandelion fairies free, descended knives through birthday cakes, Pulled Christmas crackers, snapped wish bones and sent prayers. To hold hope that I would recover. 


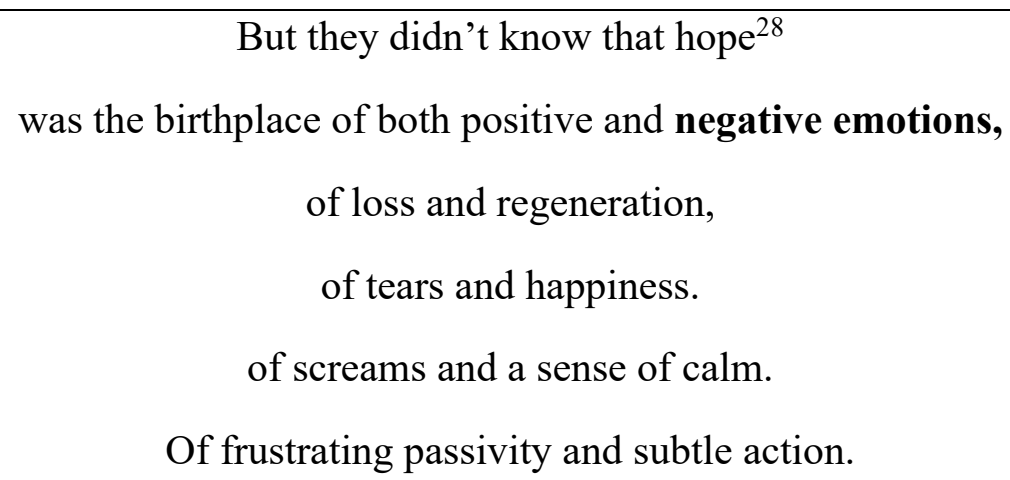

The experiences written deconstruct the positive emotions bias and binary between positive and negative emotions. It does so by:

- Describing how fostering "positive" emotions can have both negative and positive outcomes.

- Demonstrating the existence of both positive and negative emotions in an experience. When positive emotions function alongside negative emotions they contribute to the individual's emotional complexity (an ability to maintain sadness and happiness at the same time, for example). As Bodner \& Polansky (2016) state in their literature review, emotional complexity is associated with higher self-esteem, life satisfaction and other strengths.

The implications of these on my MT practice is that greater consideration is needed in pursuing goals such as "fostering hope" and ensuring certain emotions aren't privileged over others. Supporting emotional complexity might be advantageous. Playing or experiencing music that is sad or happy does not always translate to the experience of happiness or sadness etc. Music regarded as sad is often experienced as pleasurable (Sachs, Damasio, \& Habibi, 2015). Emotions are not a reliable indicator of positive or negative therapeutic outcomes.

\footnotetext{
${ }^{28}$ Hope is often a desired therapy outcome in the MT literature due to its association with "positive emotions". Maintaining hope becomes a central therapeutic concern within this understanding.
} 


\subsection{Locating examples that deconstruct the dichotomous divide within session notes/practice (ethics).}

This section will discuss MT examples that existed beyond dichotomous boundaries and understandings in an ethic of seeing the marked term as inherently valuable and its interdependence in the therapy process.

\section{Some days Marcus didn't like the rain, Some days he did. \\ Some days he both liked it and hated it.}

Marcus improvised songs about the rain, while I accompanied on the piano. He titled them, "Rain 1" through to, "Rain 10".

He often asked himself,

"Do you like rain?"

"Do you like the hailing?"

His answers explored different answers to these questions and both "positive" and "negative" reactions to the rain.

Excerpts from his lyrics follow:

"Hail extreme, everyone dies, everyone has died....thunderstorm, what happens

$$
\text { when you don't run?" (rain 4) }
$$

“It's starting to get really stronger. It's horrible, and it's terrible." (rain 5)

$$
\text { "Enjoy your sunshine" (rain 4) }
$$

"The rain is heavy once again, even more like the rain.

The rain is coming. Do you like the rain?

I like the rain. Please don't tell me it's Saturday though" (rain 5).

"The weather is cloudy and sunny,

"Do you enjoy yourself, every time that the rain stops"? (rain 5)

"Rain 6 is fun" (rain 6)

"I was terrible every time when the rain starts" (rain 6). 
"Me and sister like to play outside. The sun is up, rain is coming, that will be good as well" (rain 6).

In his rain songs there was joy and happiness, fear and worry.

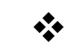

\section{William.}

Major keys were his safety net.

There he hid behind the pleasure of happy scales.

I would test the water and start with a minor chord.

"Only happy" he would plead.

In major keys I played for a further four sessions.

The positive vibe was deceiving.

His music was happy, but he was not.

His world had shrunk,

He could no longer participate in class.

I presented him with more space for both modalities to co-exist.

I played an improvisation in minor tonality, "it's your choice whether you join in William", I said, "but I need a short break from playing happy".

$$
\text { Part way through he played. }
$$

We moved between modalities, both happy and sad sounding. Sometimes both coexisting at the same time: his hands playing in the pentatonic, mine in A minor.

"How was that?" I asked

“Well, actually it sounded like my video games. It's not how I wanted you to play, but sounded cool."

In my journal, I wrote this poem after his session: 
Let's go camping in dark tunnels.

Eating marshmallows with monsters in the dark.

Listen to the language of ghost stories.

We will stay,

Boy,

We will wait

Until we are ok in not seeing a way out.

If we can feel the black around our sleeping bags,

Know all its bumps and shapes.

We will learn what it is to speak of

Close your eyes

Boy,

Rest your eyes.

They're no longer looking for the light at the end of the tunnel,

For you have learnt how to see in the dark. 


\subsection{The practices, conditions and discourses that sustained the "truth" of binaries in my practice in 2012 (truth).}

For a variety of reasons, humans in developed countries are thought to have developed a sense of being separate from non-human entities (Vining, Merrick, \& Price, 2008). Non-human entities in this section will refer to environments that are not human creations: plants, animals, insects and products of the earth and planet itself. Ideas about the non-human in my practice were based on anthropocentric concepts, they were portrayed as being separate to humans in written material. Within this view, humanist man is front and centre and put before other species and the environment. Our current politics continually reproduce the exceptionalism of humans (Puar, 2013). They are seen to have unique capacities and abilities.

I identified three discursive practices of anthropocentrism and humanism in my 2012 work that created the binary:

- The focus of the therapeutic relationship was between humans

- The therapeutic objective of 'fostering relationships with others' was limited to human relationships.

- The physical boundaries of the music therapy room

The practice examples below support the anthropocentric and humanistic "truth" that humans are separate from non-humans and that non-human presence is less important than the human aspects of the therapy process.

In MT definitions I have used when describing my work for presentations and school brochures etc., the therapeutic relationship was framed as being between therapist and client, using a strong anthropocentric therapeutic discourse. This corresponds with many MT definitions where the relationship alluded to is an intra-psychic one between two human beings (American Music Therapy Association, 2016, Australian Music Therapy Association, 2016). For example, in 2014 the American Music Therapy Association defined MT as "the use of interventions to accomplish individualised goals within a therapeutic relationship by a credentialed professional 
who has completed an approved music therapy programme"29. Kenny (2015) notes that so much of our MT literature is anthropocentric. Bruscia's (2014) working definition of MT opens up the possibility of this relationship between human and nonhuman entities. The definition states that therapists help optimise client's health through "using various facets of music experience and the relationships formed through them as the impetus for change" (p.36). He clarifies that music affords opportunities for multifaceted relationships including between a person and their physical environment.

However, it is difficult to utilise such a definition within education contexts. The key competencies that frame student's work at some schools have little room for acknowledging relationships with the non-human. The key competency of relating to others is focused on human relationships. In accordance with this, outcomes of my practice often mentioned relationships formed between human beings.

MT discourse makes it appear that the process takes place in a vacuum when the environment that the therapy takes place in is not mentioned. My practice is conducted inside a school building, that I have organised and can control the specific placement of furniture, instruments etc. (Berger, 2007). Non-human made environments are quite different to this place. I do not control elements such as the weather. The boundaries of the room limit the opportunity to interact with other environments at various levels within sessions (Swank, Min Shin, Cabrita, Cheung, \& Rivers, 2015). In Hoskyns' (2016) review of Ansdell's (2014) book, she notes a need for ecological music therapists to develop and embrace indigenous people's special relationship to the natural world, the landscape and place of experience. She felt this to be missing.

\subsection{The power effects of the dichotomy (power)}

Three effects of privileging the human aspects of therapy and separating humans and non-human entities were identified:

- It places individuals that meet humanistic ideals in an ethical state above the non-human (Lamb, 1996; Vining et al., 2008)

- The non-human's role in our wellbeing and its therapeutic effects may not be adequately addressed, stated, or thought about.

29 (https://www.musictherapy.org/about/musictherapy/) 
- It may increase the separateness one may feel to non-human entities. This may, for example, limit people's understandings of the life-threatening impact of climate change.

Individuals that meet humanistic ideals can be placed in an ethical state above the non-human (Lamb, 1996; Vining et al., 2008). In this humanistic therapeutic discourse that purports anthropocentrism, outcomes supporting autonomous, independent, rational and linguistic ways of "being human" are privileged over interdependence/dependence and disabled ways of being. Disabled people who don't meet humanistic traits can be denied inclusion in the category of human and become non-human. The main problem isn't that disabled people become "non-human" or "less human". The issue stems from the fact that humanistic ways of separating human and non-human entities makes a certain type of human more valued and those non-human like less important (Goodley, Lawthom, \& Runswick-Cole, 2014). This individualised "human nature" is therefore not only a hindrance to how the disabled are treated but it is environmentally harmful. Disability and environmental politics therefore become interrelated issues.

Due to the privileging of humanism in my prior practice, exploring ways of the nonhuman have at times been censured. For example, I had a student, Peter, who would meow when I asked him a question. Following the request of his teacher, I asked that he replied to me in English. His opportunity for learning "with" and "from" making cat noises could not be respected within humanism, as he needed to "act like a human". However, he used cat sounds when verbal interaction became challenging for him. Once they were observed for what they were instead of silenced, through the cat sounds Peter and support staff learned of his needs and ways of managing.

Non-human's role in our wellbeing and its therapeutic effects may not be adequately addressed, stated, or thought about within such binary producing discourses. Research suggests that engagement with non-human entities contributes to both physical and mental health (McCurdy, Winterbottom, Mehta, \& Roberts, 2010; Swank et al., 2015). It allows people to learn about survival and shed feelings of alienation with the realisation that they are connected to the earth and other living creatures (Kenny, 2006). For this reason, devaluing the non-human limits the potential of the therapy.

Binary discourses may contribute to the separateness one may feel to the non-human. Vining et al (2008) found that a lack of contact with non-human made environments 
and an increase in contact with human built environments may lead people to feel more separate from non-human entities. Therefore, the experience of place in therapy sessions may directly tie one's connectedness with or separateness from non-human entities. This becomes a concern, as feeling separate from the non-human may limit people's ability to understand what is happening to the earth/natural world as a result of climate change, which is ultimately life-threatening. Being more connected to the non-human encourages action.

\subsection{Personal experiences that deconstructed/dismantled the dichotomous divide: Identification of non-binary moments (ethics).}

A shortened version of a prior journal entry is presented below. There was a struggle within this entry to free myself from anthropocentric and humanistic thinking, which wasn't always achieved. However, it works towards dismantling the binary through becoming with the tree and exploring the interconnection with the non-human. Becoming animal, tree, plant, etc., is about displacing anthropocentrism. Further discussion follows the entry.

2011:

There is a large tree outside my window.

I see her as an unlikely

friend as she speaks in twigs

and moves in bends. Claps

with leaves and grew from

seed.

On first glance, could we be more different?

We didn't properly meet until I was

stilled enough to see our connection. 
Her brown leaved branches

poke through her belly: Dying

slowly from the inside out.

Inside me browned postganglionic nerves wait to see if they can turn green, so I can go.

Tree or human,

disease knows no difference.

I look for my misplaced smile out the window and find my friend.

A bird unpacks his picnic

on her limb.

She is never alone as the

wind touches her and the

clouds brush past for a hug.

She has only one spot in the

soil, but she changes colour

in different light.

She need not go anywhere

to change. 
as they put more pins on the map from going places and growing careers and families,

While I lie passively still and stunted in this room:

a hibernating bear waiting for summer as if the snow wasn't pretty enough.

Rain showers her, husband showers me.

Soil feeds her, family feeds me.

Wind moves her. People move me.

She makes depending on others look easy,

I realise that while she depends on others for some things, others depend on her.

We are interdependent. 
Bare to the elements, my

friend flexes and bends:

nearly breaking in the

Gael force winds.

Being rooted in

Wellington she has no

choice but to bear through

the sideways rain and the

early frosts.

I worry for her, for I

know of the bite of chest

pain that shivers each

breath and the swirling

nausea of being bashed

around from a force not

seen.

Will she be there

tomorrow?

But she still remains

through the storms: a

success rate of a hundred

percent.

$$
\begin{gathered}
\text { I can't help } \\
\text { but want to see } \\
\text { myself like } \\
\text { that. }
\end{gathered}
$$

Though her branches may

ache, she is there for others to

provide, shelter, shade, 
safety, and a home with her

vulnerable strength.

She doesn't judge or suggest,

she's just there.

She is my therapist.

As Virginia Woolf explained

(1930), people learn virtue from

plants.

It is in their indifference that they

are comforting.

She releases the oxygen that runs

through my lungs.

And so I watch my

friend, some days

more than others.

Sometimes it's all

and everything I can

do.

As I watch

I learn how to be a tree.

I slip from being human.

There is more on offer to me right now

in this tree way of being.

There is a struggle in this entry to move away from humanistic perspectives that define how we understand the non-human and ourselves. Slippage into anthropocentric and humanistic perspectives is easy given that they are constituted in my subjectivity. Consequently, this entry personalises the tree and gives it human 
qualities and experiences, venerating "human" understandings and embodiment. Human meaning-making was clearly a constitutive force. However, apart from minor edits I have kept its original form because it highlights the power effect of holding on to the humanistic ideals of independence, autonomy, rationality, and control. The emotional challenge of not meeting these "human" standards was evident. Feeling "less human" and denied the category of "human" become possible within these discourses. It was only when I became disabled and produced as non-human that I started to feel "less than". I attempted to reconcile this within the entry by valuing other ways of being that are suppressed within humanistic discourse. The more I valued the tree the more I valued my current self.

The entry indicates a shift away from humanism. For example, ideas about interdependence were explored, which started to change the way I related to myself and the tree. Disability demands mutuality, support, and interdependent connections with humans and non-human entities, such as the tied togetherness of the guide dog and human (Goodley et al., 2014). From watching the tree, I started to re-constitute and reposition myself.

I found the counter discourse of posthumanism, where fixed distinctions between human and non-human spheres no longer hold (Quinn, 2013), helpful. The human is not distinct, but instead coexists with the non-human in various interconnected forms. Demolishing the human-non-human divide in a posthuman way treats all ways of being as valuable and treated with importance in therapy: A "trans-species solidarity" (Braidotti, 2013, p. 67).

From reviewing my journal entry and moving towards the counter discourse of posthumanism, I became aware that:

- Non-human entities provide us with the possibility of knowing other ways of being (Davies \& Whitehouse, 1997) that move one out of humanistic ideals. Health and wellbeing do not just consist of humanistic ways of being-which are prominently privileged in humanities discourses. Many writers with illness/disability have written about this and have discussed the ways that snails (Bailey, 2010; Schuman, 2002), worms (Halifax, 2009) and dogs (Honeyman, 2014) have been of support to them during illness. They increased one's appreciation of other ways of being or acted as a proxy (a way of externalising an invisible illness). Mimicking animal behaviour may be 
respected as an opportunity for learning within posthuman discourse. Learning “"with' and 'from' rather than 'about' non-human animals...Learning within this context may be perceived as a convergence of energies" (Pedersen, 2011, p. 7).

- Human and non-human entities are interrelated and constitute each other in practice: "We are the earth, through the plants and animals that nourish us. We are the rains and the oceans that flow through our veins" (Suzuki, 1992, in the "Declaration of Interdependence").

- There can be comfort and ease around non-humans that is not present around peers or family. Emotional attachment to the non-human can be greater than that of humans. This may occur partly because for some disabled Others, being human feels alien because their way of being never made it into full humanity (Goodley et al., 2014).

\subsection{Locating examples that deconstruct the dichotomous divide within session notes/practice (ethics).}

MT examples are presented that existed beyond dichotomous boundaries and understandings in an ethic of seeing the marked term as inherently valuable and its interdependence in the therapy process. I found the work of Kenny (2014) and Hodgeson (2014) helpful in thinking about the examples below. Whilst not a selfproclaimed post-humanist, Carolyn Kenny's MT work and her strong message to "stay close to the earth" (Kenny, 2014), helped support how this can be realised in my practice. Hodgson's (2014) exploration of kaupapa Māori models of health in relation to his MT practice also had much to offer. His thesis is rich in examples of practice that support the interconnected relationship with the non-human environment. One example below demonstrates the role of a bird in constituting and exploring different ways of being.

He didn't want to explore, and I struggled to engage him in playing music.

He sat on the swivel office chair.

With arms outstretched, he vocalized,

"Shhhhhwish" as he spun around.

I reached my arms out, and said, "Swish" while flying around the room.

I then begun singing: 


\section{"Oh we are birds, \\ where are we flying to?}

Will we fly to the drum or the xylophone?

"No, piano", he said.

"Arms out wide,

Ready to fly.

Swish."

He got to his feet and started gliding around the room.

One of his hands runs up the piano as he glides towards me.

He went places as a bird that he previously wouldn't as a "human". When he got to a new place in the room he would play and explore sounds.

The group couldn't decide what to do.

"I want to make instruments", one said. "But we don't have anything to make them with", said another. "What could we find to make sounds with", I asked. "stones" one said. "Can we go outside and see if we can find stuff to make instruments with"? They asked excitedly. "Ok, get your coats" I replied.

On the muddy banks down to the playground and between the trees, cries of, "look what I found", "This makes a cool sound”, "hey yours sounds good with mine”, echoed back to me. I trailed behind them, supervising as I perched on a log: leaving them to work together to find what they were after.

After 20 minutes we came back to the music room. Hands grasping twigs, rocks, leaves, as well as rubbish left from little human hands that lay beside the earth's offerings. The following week with scissors, glue, and string they made instruments from their gatherings.

An improvisation occurred from their creations. I accompanied their instruments on the drum made from wood and the skin of an animal. Music made and produced by and with humans and non-human entities: interdependent for this moment to happen. 
Liddy wrote a song that demonstrated how the therapeutic relationship with the non-human can be held and supported within sessions. The relationship she had with her dog and the outdoors was significant to her wellbeing. The land taught her another way of being a "girl" through contact with dirt. The outdoors became part of her identity as an "outdoors girl".

'Being outside' by Liddy (9 years old)

I like going outside with Marley (dog)

It makes me feel calm,

Outside there's lots of space,

Sheep, dogs, chickens, cows, pigs.

Being with them all I am there, just there

Being with the animals.

Dirt, lots of dirt.

On my dirt bike,

Fun, going fast.

I'm not a normal girl.

I'm an adventurous girl, an outdoors girl,

A girl dirt bike rider". 


\section{Chapter 7}

\section{Finding Ladders: Success}

"Moment of success": Painting by my husband Bevan.

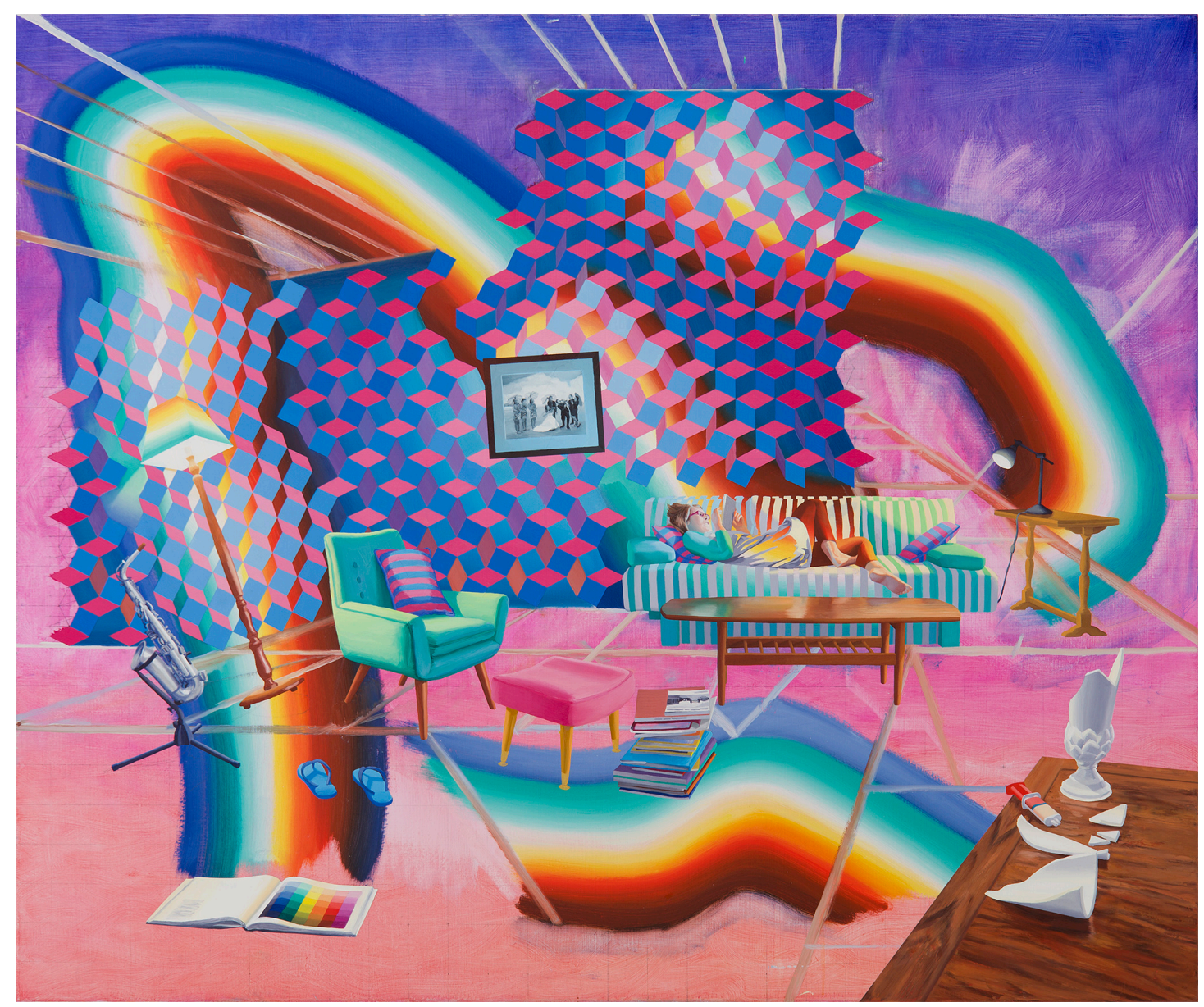

[Colourful painting of me lying on the couch-my typical $\mathrm{PhD}$ work position-reading from an iPad in a living room.] 


\section{Introduction}

It is hard to feel like a success when success isn't successful in preventing you from falling down the rabbit hole of felt failure. So, I wrote you a list to ladder a way out:

You rock when you work part-time

You rock when you cannot

You rock when you can cook

You rock when you cannot

You rock when you can get dressed

You rock when you cannot

You rock when you keep breakfast down

You rock when you cannot

Others rock when they run marathons

And you rock though you cannot

You rock when doctor's like "cases" like yours

You rock when doctors do not

You rock when medications work well

You rock when they do not

You rock when you contribute

And you rock when you do not

You rock when you have it together

You rock when you do not

Some of these things bring pats on the back, and the other things do not.

But success surrounds you; you just have to know where to look.

It can be found in moments of movie watching to get through an afternoon, 
It can be found in shared jokes and in holding someone's hand.

Find it, embrace it, go swing off the chandeliers and celebrate it all.

And in that moment of despair, where you think you are not enough,

know that not being as able in some way does not make you less.

For this ladder of a list disabled success.

How we define success can shape the ways in which one comes to experience oneself as a success or failure. The above writing problematises what success is thought to be in a neoliberal society.

When I was unwell, I found myself in a state of not knowing what success actually was. I felt a dissonance between the ways I had redefined success personally and the ways I had to describe it at work. How humbling it was, when every strength I thought I had could be reduced to weakness (Frank, 1995) and the ways I measured success became irrelevant. When I reframed success with the thought that we do not exist purely for our achievements and functional capacities, I started to see other forms of success. I also note that the two dominant disability discourses (the medical and social models) couldn't always capture or adequately frame my successes. There were times when medical intervention could not relive my struggles or social change. My experience, in these moments, fell outside of medical, social and medical-social hybrid models of understanding of success.

This chapter looks at how success is determined and defined in the context of, the previously coined, "special" 30 education in New Zealand (NZ) and the impact of this

\footnotetext{
${ }^{30}$ Many disabled people refute the term "special" and prefer "disabled". Using words such as "special" implies that the word disabled is too terrible to say, further stigmatising disability. I recognise the shift in the Ministry of Education's terminology that attempts to be more inclusive. Using terms such as, "learning support"and "inclusive education". However, at the time of writing, the term, "special" continued to be dominantly used by schools, teachers, and parents. Having noted this, I will use the Ministrys new terminology of "supported learning" throughout the rest of the chapter.
} 
on my practice. It explores alternative ways of understanding success. Success in education is heavily influenced by neoliberalism, which draws on discourses of humanism, measurability, predictability, and standardisation to sustain it. Capitalist imperatives call for control, standardisation and predictability in the market and the humanistic man, with autonomy, choice and competence, to be the consumer. Leading to a "compulsory able-bodiedness" (McRuer, 2006). These dominant discourses often determine how success is defined in education, which in turn often governs understandings of success in music therapy (MT) practice within this context. How people with disabilities were represented in my MT paper work pre-2014, had been influenced by the ideologies of autonomy, progress, self-reliance and the modern subject. This demonstrates the effect of external pressures from these discourses enforced through neoliberal government policy.

Since beginning my practice in 2006, there has been increasing demand to provide evidence of the success of the work, particularly within secondary schools. Due to the differences in primary and secondary school settings, the following analysis is based on my work in secondary schools only. There has been more flexibility in framing success in the primary schools I have worked in, with less demand for evidencing success in specific ways, possibly due to the different funding pools and processes. Because the secondary schools I work at are fund-holder schools ${ }^{31}$, the Ministry of Education (MoE) routinely reviews my practice. The review process for the specialist service standards, that regularly takes place, is used to assure the MoE, who provides the bulk funding, that specialist services are delivered in line with the standards and promote achievement. Therefore, I must talk of success in the ways they have outlined to meet my obligations as a therapist funded by taxpayer money. Generally, there seems to be preference for interventions that produce predictable and measurable outcomes. Funding is competitive in some institutions. This results in fluctuating hours, cuts to inefficient working practices, and a need to prove that money has been well spent. Uncertainty around job continuation is prevalent, so mapping success becomes important.

\footnotetext{
${ }^{31}$ Fund-holder schools get the funding for the ORS students on their role to then employ/contract therapy services and specialist teaching and support staff.
} 
This environment contributes to the need for the MT profession to develop standardised tools to measure and record client changes. Roman (2016) reflects that perhaps the profession's difficulty in developing measuring tools of success indicates the complexities that music therapists have in evidencing the work, particularly in quantifiable ways. Furthermore, I propose that there is difficulty in determining what constitutes "success".

In this chapter, three interrelated "truths" produced in this neoliberal environment will be discussed that constitute "success" for students with disabilities in educational settings. In all three, success is defined by standardised norms:

- The ability to meet normalised standards determines the level of success

- "Success" can be measured

- "Success" is predictable and therefore can be planned

Power effects of these "truths" will be discussed as well as ethical considerations. Each "truth" will start with a journal entry in which I have personally struggled with, problematised, and attempted to resist these "truths" in some way. The analysis that follows, links with these entries. Journal entries are included because they were where I worked through the "truths" being at odds with my MT practice and personal understandings. I took these entries to MT supervision to develop further understanding.

The three "truths" form the main headings in this chapter, which are structured with the following subheadings:

- Journal entry-used to highlight the points discussed within the following three subheadings

- Truth

- Power

- Ethical considerations 


\section{2. "Truth" 1: The ability to meet normalised standards determines the level of success}

\subsection{Journal entry}

Dear ill body, I Surrender.

For years I used the words given to me. These words I used to make sense of this mess. You're a "fighter," I was told, and so that is what I became. And you, you were my Darth Vader. The one I feared, despised and longed to turn back to the good side. For those raspy breaths in your presence, whilst comical on screen, felt as raw as they sound in real life. So, I built armour from chopping $3 / 4$ of my work hours and a stress less policy. From compulsory bed routines and a diet greener than my nauseous stomach. I swallowed swords and artillery all shapes and colours from pharmaceutical companies all around the world. And pierced your skin with needles, pouring liquid weapons directly into your veins. I aspired to be like everyone else and would one day "win the battle" (swain g cameron, 2002).

Yes, we have been at war for some years now and defeat has blown my candle out. I no longer want to be a soldier obtained by conscription, where bravery must be upheld to play the part well. For there are times when I am scared for you and I don't want to shove my face behind the mask of a soldier. Magazines and newspapers are littered with statements of, "a brave fighter of__ " or "lost the battle to___. Praised are those who continue to fight.

Using war as a metaphor for chronic and incurable illnesses sentences one to lifelong failure: head hanging in the shame of no complete victory and drowning in the burden to continually keep up a soldier's fight. Of carrying hate, as it is what feeds war, and giving up life to dedicate all hours to the cause of remission.

Body, this is not the life I wish for us.

So, from today I will not fight you. Nothing good comes from violence. If I do not fight, I cannot win, and I cannot loose. It is simpler this way. 
Kinder, perhaps (Marshall, 2015). Whatever the new metaphor will be, I know this: When your triple figured heart rate makes me too dizzy to stand, I will lie on my back and show you how pretty the sky is. When you ache from central core to every outer inch, I will hug you in the arms of an Epsom salt bath.

Someday we may get better. Till we do, let us learn to live as if living might matter (Ferris, 2013, p. 550). Through love, not war.

\subsection{Truth}

I will discuss how disabled people are assessed according to conventional standards about what constitutes normal and desirable outcomes (Stone, 2005) and how they are encouraged to "fight" (self-govern) to reach able-bodied standards. I examine how capitalism, humanism and metaphors of illness and disability construct this discourse of standardisation that frames success in education and consequently some of my MT practices.

The normative ideal of mental and physical wholeness is privileged in society. As such, the normal developing child is at the epicentre of educational systems. The NZ Curriculum is based on an ableist body/mind, which educators in supported learning need to adapt for people with disabilities ${ }^{32}$. Specialist services need to be linked with the NZ Curriculum, which, when met, validates success. In August 2016 (the time of writing this chapter), Hekia Parata (Minister for Education) announced that schools receiving $\mathrm{ORS}^{33}$ funding would need to show how students are progressing, which would be measured through NCEA and National Standards (Moir, 2016). Therefore, the aim of supported learning within discourses of standardisation is for students to overcome their disabilities or to cure them, which is neither possible or desirable (Straus, 2014).

\footnotetext{
${ }^{32}$ One examples is the Blind and Low Vision Education New Zealand curriculum.

${ }^{33}$ Ongoing Resourcing Scheme (ORS) = Funding for disabled students in NZ
} 
Standardisation draws on the sub discourses of capitalism and humanism to form the normal standards. The capitalist notion, that money determines the value of something, is present within the NZ School Curriculum.

One parent I talked to was shocked that her severely disabled daughter had to have a career plan as part of her education.

The MoE claims that career education and guidance seeks to "enable all students to realise their full potential as individuals, and to develop the values needed to become full members of New Zealand's society" (Ministry of Education, 2009a, p. 5). It further suggests that becoming resilient career managers is essential in enabling students "to live full and satisfying lives and contribute to a sustainable future for Aotearoa New Zealand" (Ministry of Education, 2009a, p. 7). Such statements imply that a life with paid employment is a happier one and that we are only "full members" of society when we do. An individual's social worth rests fundamentally on their waged or non-waged status. If students grow up to take more money than they give they become social problems without full citizenship. An ethics of minimisation and normalisation of disability is present in this conception of citizenship (Campbell, 2006). Success, therefore, is framed by use values that are based on normalised standards. Interestingly, whilst disabled bodies are deemed of less worth, capitalist consumerism, where demand is manipulated to the advantage of sellers, capitalize on disabled bodies. For example, people with chronic illness are large consumers of pharmaceuticals and disabled people consume assistive technology.

The drive for specialist services to increase independence reinforces the expectation that disabled people should endeavour to avoid needing financial or personal assistance (Kielhofner, 2005; Zola, 1993). One of the key questions during the specialist service standards review that I am required to answer is: "How do you ensure that individual programme plans are current, contain meaningful learning outcomes, and promote achievement and independence?" (Ministry of Education, 2013, p. 4). Examples like this are plentiful.

Capitalism also drives what is considered therapeutic success outside of education. Rehabilitation therapists are required to show how their services will reduce costs 
over time by reducing disability and enabling the person to be more independently productive, lessening social/economic burden (Kielhofner, 2005).

One client I worked with was denied funding for rehabilitation therapy services through ACC, as it was thought that receiving therapy was not likely to increase her functionality. It was deemed that such resources were better allocated to those who may become more financially productive. She was given carer support services only.

This "truth" that meeting normalised standards constitutes success in education is therefore a wider phenomenon impacting practice in other settings.

Supporting economic policies of capitalist societies are the idealised essentialist humanistic traits; the autonomous, competent, rational self (Erevelles, 2011). Much of education uses traditional humanistic discourse, which inscribes these characteristics as pertaining to the "good" citizen. The notion of the autonomous self and rationalised schemes have underpinned how we have come to understand others and ourselves and therefore how we evaluate people and lives (Rose, 1996). In NZ education, autonomy is evaluated under the key competency of "managing self". Autonomy also has a place in many MT practices, such as, autonomy is one of the profiles in Bruscia's (1987) “Improvisation Assessment Profiles". Carl-Roger's person-centred therapy, a founding figure in the humanistic school of psychotherapy, has indeed been central to my practice for the past 10 years. Many MT approaches are underpinned by humanism, even in more contemporary models it is solidly present (Ansdell \& Stige, 2018). For example, ROMT claims that it is deeply rooted in a humanistic tradition and in humanistic values related to humanity (Rolvsjord, 2010).

If capitalist and humanist discourses constitute the standards of normality, military metaphors encourage one to strive towards meeting them, to "overcome". The military metaphor of disability and illness is present across contexts. It is often believed that disability diminishes one's quality of life (which has been challenged vigorously by disability theorists), and therefore disabled people "fight" to overcome them (Carlson, 2013; Honisch, 2014). "Overcoming” disability communicates that in their disabled state they were inferior (Hadley, 2017), less successful. 
I remember a well-intentioned family member saying to me during a remission that, "it was good to have the old Carolyn back". Implying that "disabled Carolyn" was not as good/inferior. I tried to explain that the pre-illness Carolyn will never be back: I had changed and learned too much to not be different.

Disabled people are enticed through social rewards to "fight" to meet these standards. This metaphor is prominent in both health and disability spheres. In the 1980 s the military metaphor came into wide use in medicine: the discovery of bacteria led to them being described as invading or infiltrating (Sontag, 1978).

In MT, "resistance" can be referred to when the person chooses not to play music with the therapist and may employ "defensive" strategies. Rolvsjord (2010) notes that in analytic MT, there is a focus on conflict. The regulation of patients' defense mechanisms is a particular focus (Priestley, 1994; Rolvsjord, 2010). "Intervention" is also associated with military language and implies that action by others is being taken (Rolvsjord, 2010). Intervention is referred to in MT and used in some older definitions (Bruscia, 1998, p. 20). Within the NZ Standards of Practice, "intervention" is one of the categories required in demonstrating a music therapist's competence (New Zealand Music Therapy Registration Board, 2012). The metaphor is present in MT papers, for example, the title, "Battling illness with wellness" in Solli (2015). Indeed, it has been celebrated when clients adopt an identity as a "fighter" during the MT process, as seen in the Kennelly (1999) example given by Baker (2017). Thus, demonstrating music therapies role in constructing identities with this metaphor. Similarly, the metaphor of "wholeness" is often used in MT discourse to stabilise the incomplete disabled figure and give direction.

\subsection{Power}

I have identified four power effects of standardisation:

- People with illness/disability are excluded from success status and are devalued when their successes don't fit within neoliberal humanism.

- The focus on standardisation reduces the cultural tolerance for human variation and vulnerability (Garland-Thomson, 2013). 
- Standardisation and accompanying discourses, particularly military metaphors, position people as either triumphant winners who overcome disability/illness or abnormal losers in need of pity and fixing.

- People with illness/disability can be "at war" within themselves to meet normalised standards.

Any attempt to standardise must come to terms with what it excludes (Kenny, 2006). The material conditions of capitalism produce disability as "lack" (Erevelles, 2011) and therefore excludes it as a successful way of being. Due to this, as embodied in the above journal entry, "the corporeality of the disabled body is constantly in a state of deferral, in a holding pattern, waiting for the day it will be not just repaired but made anew (cured)" (Campbell, 2008a, p. 157). Humanistic ideals of autonomy, control, rationality, and independence position people with disabilities as (ir)rational, (non)autonomous and (non)competent, leading to their exclusion, denial of full selfhood, and status as unsuccessful human beings (Erevelles, 2011). The dignified position of oral speech in humanism also contributes to exclusion. Voices deemed not rational and intelligible are judged against normative standards in an attempt to maintain the boundaries of human (St. Pierre, 2015). This links with the power effects discussed in chapter 5 pertaining to the human-non-human binary divide. The fact that disabled people may contribute to society in other ways than paid employment, such as through artistic endeavours, as supporters or witnesses', and giving joy or compassion to others, counts for little to nothing (Thomas, 2007) in determining success.

Illness/disability have no or little importance in success when it is framed by standardisation. The capacity to be unwell or disabled struggles to be valued and held (Roman, 2016). Halifax (2009) demonstrates the governmentality of this when she states, "I'm a failure again. My body has gone its own way...I am broken again, and you who are so good at fixing can't fix me" (p.124). This may impact on therapists not valuing students and accepting them as they are in the here and now. They may pursue an overcoming of disability instead of supporting one in learning to live a content and full life with disability (Nabors \& Pettee, 2003). This acceptance is different to allowing people to be unsupported in inadequate services (Roman, 2016). The drive to overcome can impact negatively on the focus of sessions. For example, 
the maintenance of walking might not be the "best" option for a person, even if it is a standardised goal. A wheelchair might be a more feasible option for mobility and not necessarily a failure (Eisenberg, 2012).

When standardisation and associated metaphors frame success, two positions are made available to people with disabilities/illness: winners who overcome their disabilities to embody more normalised ideals and losers who do not, they lose the "war". As mentioned in the above journal entry, this can cause people with disabilities/illness to be at war with themselves, trying to, and often failing at, meeting normalised standards. It is exhausting to keep fighting, especially when the message that you must change, you are not getting it right, you are not progressing, squashes self-esteem (Prizant, 2015). Knowing that no matter how hard one tries they won't succeed in normalised ways, causes frustration, unhappiness, and anxiety (Prizant, 2015). Such a frivolous pursuit can also be financially draining (Prizant, 2015). Like the idea of overcoming, the standard of wholeness is arguably an unobtainable corporeal aesthetic that refuses to esteem differences (Campbell, 2008b). Therefore, disabled people may find themselves, more often than not, in the position of failure.

\subsection{Ethical considerations}

The above analysis suggests that an ethic of practice that keeps extending what it means to be human, accepts illness and disability, and interrogates metaphors and practices that perpetuate the idealisation of normalised standards, might be advantageous. In order to change how success is understood, we need to question the kind of human currently valued by society. Thus, raising the ethical question, "what is a good life" 34 . Some music therapists have already developed thinking around this, such as Kenny (2006) who suggests that we need to suspend and question our judgments on what a life should be.

Further possibilities/ideas for music therapists to counter some of the effects of measurability that restrict disabled people through practice are raised below. 'Disability asks of ability: 'Is that all you've got: ability?' 'Are you really that superficial in what you consider to be worthy of merit?' and 'Is individual achievement all you desire and live for?'” (Goodley et al., 2014, p. 3). When we ask

\footnotetext{
${ }^{34}$ I have underlined ethical questions that stuck out for me.
} 
such questions we can begin to supplant neoliberal ideals with an "ethos of care, interdependency, and slowness" (St. Pierre, 2015, p. 341). Valuing this change of pace was acknowledged in the journal entry above. Thinking of our humanness in terms of interdependence, mutuality and interconnection (Goodley et al., 2014) may extend and expand what it means to be a successful human. The counter discourse of posthumanism might be helpful here in eschewing individualistic successes of communicative purity, autonomy, and self-mastery (St. Pierre, 2015). Thus, making room for a relational and distributed form of success. However, Goodley and Runswick-Cole (2014) do caution that there are times when it is important to embrace these normal standards. As they say, "after all, like them or loathe them, these phenomena undergird the language of citizenship, law and humanity" (D. Goodley et al., 2014, p. 5). Accordingly, I am not proposing that such successes are "bad" or not worth pursuing at times, more that they are not the only things worthy of acknowledging as "success". Other forms of success would do well to enter more of our consciousness in education.

Given the stronghold of normalisation, humanism and capitalism within this context, how do we do no harm? The position of music therapists as outsiders might offer some potential (Annesley, 2014; Roman, 2016). When we are not fully embedded in educational practices, we can perhaps more easily take a position of resistance to ways of thinking of success that are exclusionary and unhelpful for disabled people. Indeed, holding another viewpoint and agenda has always been active and central in my practice, despite the need to conform at times for funding continuation. As Annesley (2014) explains, starting with a schools expectation to "fix this kid" might be a necessary starting point, but there is also a need to take the child as they are. In order to do this, it is crucial not to "collude with the institutional fantasy-there is something wrong with this child that the therapist will cure" (Annesley, 2014, p. 41). Therapists therefore must necessarily fail at some level in becoming part of the educational institution. This failure creates space for accepting challenging situations, uncomfortable feelings and pain as contributing to success. We can then accept people's disabilities, illnesses, difficulties, struggles, limitations, as part of a rich life and not see these aspects as failures of our therapy or considered undesirable outcomes (Annesley, 2014). 
More weight needs to be assigned to focusing on alternative successes. As Schuman (2002) states, "what mattered had to be what mattered to me-not the things healthy people could do, not the things I could no longer do, but the things that were left for me to do, such as observe a snail" (p.33). This relates to my connection with the tree in the previous chapter. Getting well, as Wall (D. Wall, 2005) champions, may be less about fighting illness than of counter balancing it with healthy aspects of our lives. This indicates that we cannot project what is important for another person's life based on normalised standards nor call for a higher functioning level (Kenny, 2006).

Overdetermined metaphoric uses of disability and illness distort or delete the experience of people with disabilities (Garland-Thomson, 2005). They perpetuate concocted fantasies about being ill. Sontag (1978) writes that the most truthful understanding of illness, and the best way to manage it, is one resistant to metaphorical thinking. However, because pain is inarticulable (Scurry, 1985), metaphors are sometimes needed to convey what is invisible (Pafunda, 2011). But they are not meant to be permanent or generalisable. It is a practitioner's ethical responsibility to be self-critical and reflect on the metaphors they use (Diekema, 1989; Fleischman, 1999) as it is necessary for clients to create their own language. There is no other than their own to be accurate (Kuppers \& Marcus, 2008). Music therapists like Randi Rolvsjord, who have drawn attention to the issues of military metaphors within practice, have set some foundations for such an ethic. 


\section{3. "Truth" 2: "Success" can be measured}

\subsection{Journal entry}

The journal entry draws on Te Whare Tapa Whā model of health described and developed by Sir Mason Durie (1985). Concerns around contemporary psychosomatic constructs only measuring a person's health from physical or mental states led to its development (Durie, 1998). The WHO model of disability/health was also found to be wanting (Durie, 1985). Te Whare Tapa Whā understands health as a four-sided concept: te taha wairua (spirituality), te taha hinengaro (thoughts and feelings), te taha tinana (the physical side), te taha whānau (family) (Durie, 1985). These four aspects are interconnected.

Key:

Red= Whānau

Blue $=$ Tinana

Green $=$ Wairua

Orange $=$ Hinengaro

I met with the Māori advisor.

She asked me:

How do you keep your Tapa Whā intact?

I wrote my reply before our next meeting:

The foundations were laid when

Whānau held me,

Body rocked to a lullaby,

With unquestioned faith that I would be watered, as the first forgotten thoughts were formed. 
The wood matured as I,

Gave people giggles,

Chased after my slinky,

Jumped higher on the trampoline to see if I could join the fairies,

Whilst counting how many before 'ready or not'.

Four walls were built:

Whānau,

Insulated with Nelson holidays, water fights, sorry cuddles, dinner debates.

Tinana

Braced with plates of green, glasses of water, tapping feet, skiing slopes.

Wairua

Painted with Mount Cook Treks, outdoor sleepovers,

Monarch's wings, musical awakenings.

Hinengaro

Plastered with plays and writing, piano and violin, listening to life lessons, colouring in.

Built, but fragile to the elements:

A tornado could blow this whare in.

So, the four walls stood meeting at the elbows,

talking with each other to hold one another up.

But Tinana, the dancer of medals, could no longer stand.

The others took her weight: Cracks spreading and nails popping.

I tried to cover them with my hands.

I sat in the garden with my feet buried in the soil to grow more Wairua 
I found an online Whānau for others like me, for when my driveway was my outer limit.

In PJs I applied to do my doctorate, to keep Hinengaro busy.

Tinana was touched with needles and Epson salt baths.

Western medicine did not give my whare a permit.

While they tried to bring it up to code, it did not measure up, for they only checked one side.

They care for Tinana.

I am a NZ European woman, but I knew one wall was not enough.

My house has more walls too.

As the IV pump churns, I work on the homework of how I keep my Tapa Whā intact.

under this stiff white sheet, 1 feel uncertain as to whether it is, For I struggle to measure it.

How can I when it depends on where I stand to view my whare.

From the outside it's a construction site; inside it is shelter.

Perhaps health is always a construction site? Needing constant maintenance and care: Messy is what my health looks like.

I keep the rain out by spreading plaster on all the walls as I play music and go to Pilates. I brace the walls with extra planks as I catch waves and stop to feel air come and go from a depth I'm still discovering.

While I'm renovating,

Tinana gets to her knees, stands briefly, and then lies down again.

She repeats, repeats, and repeats this.

To the measuring tape of norms, she is more impaired and less intact. 


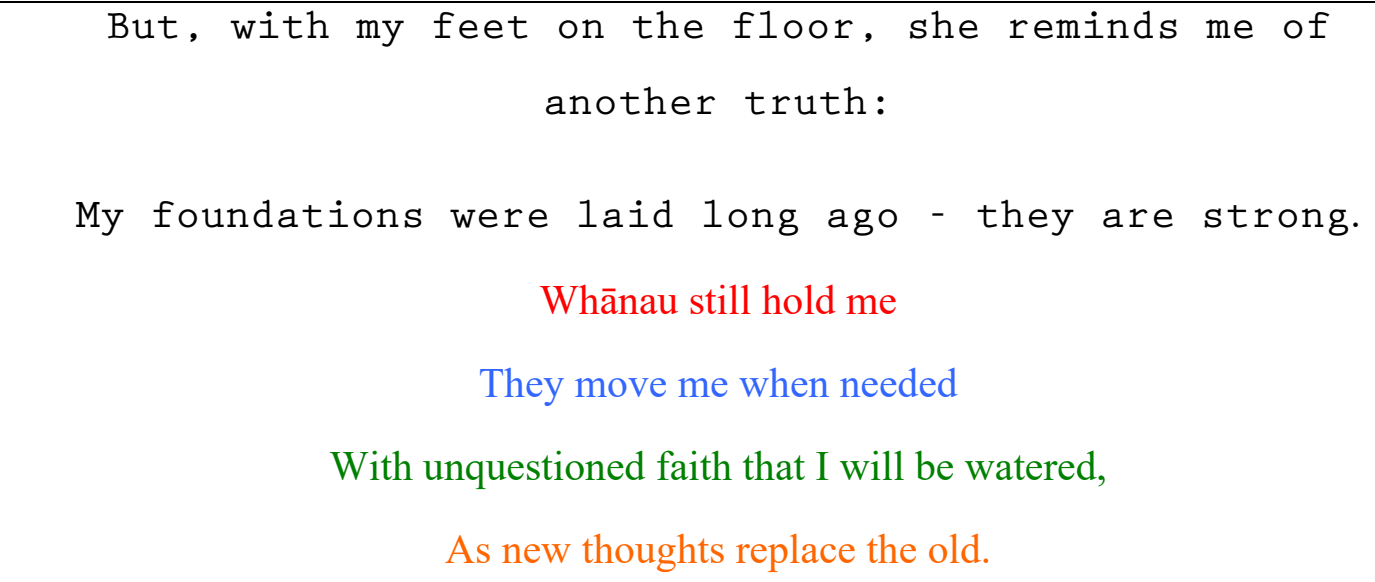

\subsection{Truth}

There is a double focus in this section: the belief that success can be measured, and what exactly is measured in supported learning in NZ. Both aspects create the discourse of measurability in education. I will discuss how success based on normalised standards is supported by the "truth" that it can be measured. Discourses of measurability in supported learning rely on student actions, endurance (active participation), and measurable responses (Ministry of Education, 2013). What seems to be missing, or less visible, in measurements of success are aspects that do not lend well to quantitative or specific measurement, such as spirituality and those which are associated with inability: passivity and inaction.

The above entry was helpful in locating what was missing from discourses of measurability in education, illuminating its constitution. As well as aspects like spirituality, it also highlighted that success is often individualistically framed compared with Te Whare Tapa Whā model (1985) where ecological measures are present.

Changing the definition of health/disability from a medicalised western perspective to the Māori model of Te Whare Tapa Whā, moved me from struggling to achieve a sense of health (western medicine) to having a sense of health (Te Whare Tapa Whā model). 
The journal entry problematised measurements as being fixed predictors of success. Sparked by the entry, I sought to uncover how success is measured within supported learning education to expand what constitutes success for disabled people in this setting.

In the programme planning section in the specialist service standards, SMART goals (specific, measurable, attainable, realistic/relevant and time-based goals) are suggested as an indicator of a successful service. An example of a SMART goal: "Melissa will choose between two instruments using PECS three times each session". SMART goals rely on responses that can be measured and these are therefore given importance over other aspects, such as spirituality, which do not lend themselves well to being measured. Churchill \& McFerran, (2014), looked at assessment tools for persons with severe to profound multiple disabilities, and found that spirituality lacked mention in prior assessment tools. Furthermore, Tsiris (2016) illuminated that some therapists had concerns about associating spirituality with MT, due to the possibility of weakening credibility. Indeed spirituality, which is well accommodated in Te Whare Tapa Whā, is poorly accommodated within SMART goals. Spirituality is just one example: I had a teacher say to me "yes, but how do I write this as a measurable goal?" when we were discussing a musical area of focus for a student attending a mainstream music class. Some aspects of music, such as the ways in which someone perceives or appreciates music, can be difficult to measure. It is interesting to note that SMART goals are also present in MT with other populations, such as neuro-disability (Daveson, 2008).

Discourses of measurability rely on active responses from students. In a presentation about key competencies success rubrics (Madge, 2010), the competency of participating and contributing described students who take a passive role in class as being "novice" and those who actively participated as "expert". The more active they were the more successful they were considered to be. Therein lies a dichotomous understanding of active and passive learners. To show that learning has taken place, the learner must do something that is different from before. This endorses the virtues of accomplishment, boldness, winning against the odds. It is a struggle to find examples of students passively experiencing something as an indication of success in IEP's as it instead indicates the failings of silence, invisibility, disquieting range of emotions and failing to improve. Active responses require endurance and strength, not 
rest and stillness. Relying on strength to gain strength. This bolsters the belief that endurance creates success, that we should keep going despite the aches and fatigue in order to live well. The reality of the ill/disabled body in needing to invest in loss and stillness to feel a bit better is excluded in this truth making (D. Wall, 2005). Examples of the kinds of language I have heard surrounding those who do invest, even if unwillingly, in loss and stillness are that they have given up, are lazy or lack motivation. Discourses of measurability are therefore based on the ablebodied/minded.

It is not only client success that is measured by active engagement and responses. Registered music therapists in NZ need to show how many professional development hours they have done. There is a lower limit indicating the least amount of hours needed for successful competence but no upper limit. This gives the impression that the more PD actively undertaken the more successful a therapist's practice will be. Down time spent not working, self-care, is not indicated as important to evaluate in the therapist's success of being fit to practice.

\subsection{Power}

There are a number of power effects that result from the truth that success can be measured through student actions, endurance (active participation), and measurable responses. They all relate to the fact that the discourse of measurability in education creates a hierarchy of success markers that celebrates some and overshadows other important factors that contribute towards broader understandings of success. A Eurocentric "normal" way of being is privileged in the measurability discourse, that of the active, doing, stable able-bodied/minded person, and some disabled ways of being are suppressed:

- Passive successes (such as receptive, listening, understanding, spirituality, moments of beauty, stillness) may lack recognition or are sacrificed to adhere to more measurable funding criteria.

- It may become challenging to work fully with regression (Roman, 2016), or to invest in loss, within pre-set measurements of success.

- Success based on particular responses (such as in SMART goals) may overshadow unspecified successes and other contributing factors. 
- Ecological measures lack consideration ${ }^{35}$

Because discourses of measurability in education rely on active responses/participation, passive listening to music may be less privileged in evaluating the 'success' of a session. Although I work with students who often have high health needs and complex disabilities, it can be challenging to formally acknowledge moments of necessary stillness or aesthetic experience as worthwhile when discourse positions them often as being immeasurable. It raises questions of how do you measure beauty or an aesthetic experience? ${ }^{36}$ Would you want to even if this were possible, considering it would lose some of its magic. Despite the challenge of being unable to accurately measure some moments, there are profound benefits to not doing.

One student became unwell towards the end of our sessions together. She wanted to just sit and occasionally listen to me play some music, sometimes preferring silence. Her measurable IEP goals were not achieved and she was considered by some teachers as "passive" and "lacking response". This suggests "failure" or that the therapy wasn't "working”.

However, Wall (D. Wall, 2005) explains the benefits of not doing and silence when she says, 'I'm too ill to talk, I need to do nothing but rest. I need you to be silent too, to join me in a restful quiet. In silence, the terrible disjuncture between myself and others...is whittled away. To be tended in silence is to be joined" (p.116). Discourses of measurability in education struggle to allow people to be successfully passive. This is due to framing active and passive responses as a dichotomy and as such, passive listeners struggle to be seen as active participants in education. ${ }^{37}$

Another student I worked with preferred to listen to the group session in the adjoining room. Her mother let me know that she sings the songs she hears at home. Therefore, whilst she was not considered an "active" group member in the

\footnotetext{
${ }^{35}$ Discussed later in this section

${ }^{36}$ Aesthetic experience refers to the personal subjective response to beauty or other aesthetic qualities of an event, object, or phenomenon.

37 This creates a delimma in education in the support of students with degenerative conditions.
} 
sense of making music and being physically present, she was still actively learning from the comfort of her own room and therefore was an "active" member. However, during group sessions, there was no way to measure her success.

As previously discussed, proof of the therapy's effectiveness is required by the ministry through the privileging of active playing and being present to contribute.

Relatedly, how music supports spirituality and offers moments of beauty are often thought of as less essential in this context due to their immeasurability. Beauty is often considered a "frill, a token gesture" (Kenny, 2006, p. 34), as its value has been lost. In MT practice, the concept of beauty is often neglected (Kenny, 2006). However, recent approaches seem to foster greater importance of aesthetic factors (Aigen, 2008). Wall (2005) cautions that it would be a mistake to think of beauty as a luxury, the first thing to be disposed of, because the more ill and pained she was the more essential it seemed.

Like Wall, I too craved beauty as a way of counter balancing illness with healthy aspects of life. I devoured poetry and felt bolstered by an unexpected dumping of snow for a week after it had melted. Beauty is a need, it can be a way of gathering strength (Kenny, 2006) and can alert and stimulate (D. Wall, 2005). For example, one student had taken a keen interest in a tambourine that had patterns in primary colours. She was excited by this tambourine and liked to draw it when she got back to the classroom. She took it with her during difficult transitions. Her teacher asked me, "what is it about the tambourine that makes her engage so well'? I said that I suspect that, to her, it is thing of beauty, much like a bunch of flowers.

This discourse of measurability fosters the idea that active ways of being are more desirable. The richness and benefits in just being not doing, in listening and not making a sound, in watching things dance while still, are harder to appreciate within this context. They are not the "peacock" display of active participation that can capture change through a measurable phenomenon. These moments took on more meaning for me when I was unwell and were part of my own "success" in managing illness. 
The landscape changes around me, and different birds soar the marshmallow sky. I am in high spirits sitting and watching it all go by. With my body so still, I see each ruffle of bush as the wind breaths. Although I am not moving and I am not doing, change is happening, and I am there with it. It moves me.

This inaction, not pushing my body past its limits, this "giving up" while my body has "fallen apart" positions me as failure when success is based on active measurements. Privileging action sustains the idea that giving up and accepting that one must not do is easy, that falling apart is bad, and that true strength and success comes from not giving up or falling apart.

Immeasurable aspects of therapy also link with the second listed power effect, that working with regression can be seen as a negative step backwards. Measurements need to be precise and become inflexible. You either achieve within the measures of success or you don't. The lack of flexibility doesn't allow for changing states and for working with regression for success. Students may move from achieving within a particular measurement to not achieving due to the changeability of their condition, which is poorly accommodated in such targets. Pupils with profound disabilities do seem to be particularly poor consumers of SMART targets. They can take a long time to move onto the next measurable skill, or loose a measurable skill, but that does not mean they are not learning or that change isn't occurring (Lacey, 2010).

The third power effect listed is that the focus on particular responses (such as in SMART goals) may overshadow unspecified successes or take into account other factors that hinder or help the particular. For example, if a student was experiencing anxiety that was impacting her learning, SMART goals might only specifically measure whether she could or could not complete a set task. Therefore, not giving insight into any shifts she had in her experience of anxiety during the task and whether she became distressed later in the day from completing the previous task, affecting her learning thereafter.

The last power effect is that ecological measures can lack consideration in justifications. A student's funding is individualistic and therefore the measurements of services for that student often are too. As demonstrated in the example below, not considering ecological measures and only relying on individual responses impacted 
on decisions around whether a student should continue therapy. When therapists are to justify their services for an individual student, this creates a predicament.

She is still, the drum rests under her twisted hands. She is watching, looking at the others noisily playing. Should she be in this group? I have asked myself repeatedly to no avail. The others actively play instruments and sing. She witnesses their changing music, movements and creations. I look for any signs of distress, or joy, or anything in-between. She is difficult to read. As her physical actions are minimal, there is little to mention in my notes. Data falls short of why she should remain. Based on her responses, I cannot measure whether or not this group is beneficial for her. However, it just wouldn't feel the same without her. Another group member likes to cover her in the cloth for the hiding game. Another passes her instruments, even though she cannot play. Her calmer presence shows the other students going full throttle another way of being. She has a role that is different but no less important. At her IEP I struggled to justify the use of her individual funding for MT when the best measurement I had was what others did with her in mind.

To summarise the discussion of the power effects, a "normal" way of being is privileged in the discourse of measurability in NZ education, that of the active, doing, stable able-bodied/minded person. Disabled ways of being, ecological factors and other aspects of disability/health, are suppressed.

\subsection{Ethical considerations}

The main ethical consideration is that dominant discourses of measurability in education devalue disabled ways of being. Those with disability struggle at times to be seen as successful when they don't meet normalised measurements. This raises the ethical question: "Can and should all successes be measured?" Indeed, the inability to be, needs to be recognised as a way of being, and the unable immeasurable self to be accepted (Carel, 2013). Foucault's idea of success would be that we resist putting people into measurable standardised boxes. Flexibility, spirituality, beauty, passive listening, and stillness need consideration and elevation in MT practice to counter the 
devaluation of disabled students created by measurability discourses in supported learning education.

Receptive MT methods, such as guided imagery and music (Bonny method), music assisted relaxation, music and imagery (Grocke, 2016), can help to counter the active participation privileged in discourses of measurability in education. Receptive methods aren't new to MT. They have been called passive in the past, however, with the acknowledgement that physiological and affective involvement are more than passive, the term receptive has become popular (Grocke, 2016). In developing my thinking around how to manage issues resulting from measurability discourses, I found four other counter discourses to be helpful: 1) Te Whare Tapa Whā, 2) SCRUFFY targets or narrative assessments, 3) The field of play, 4) Small's construct of "Musicking” (Small, 1998) and Elliott's (1995) concept of musicing.

Indigenous perspectives have much to offer, especially in thinking about, "what is the value and use of aesthetics"? Both Te Whare Tapa Wha ${ }^{38}$ and Kenny's field of play offer spirituality and beauty a prominent place in therapy. They raise an ethic of creativity and beauty. I note that achievement is not an idea used for constructs in the field of play (Kenny, 2015). Kenny (2014) describes music as a form of beauty and the need to bring it to people in pain and struggling. Aesthetic is the first field of play. This is based on the Navajo way that, "as we move more toward beauty, we move toward wholeness (Kenny, 2014, p. 4)". Māori models of health also encompass beauty within spirituality (Hodgson, 2014). Although my understanding of Māori health models is still developing, my appreciation for spirituality and beauty in practice feels more nurtured within these. It made me think how differently educational plans would look if they had Te Whare Tapa Whā as the foundation. Currently the secondary schools I work at do not formulate IEPs in this way, however, there is the possibility to do so as specialist service standards documentation mentions that specialists may use Te Whare Tapa Whā.

SCRUFFY targets (Student-led, Creative, Relevant, Unspecified, Fun, For Youngsters) offer flexibility (Lacey, 2010). SCRUFFY targets are still targets, but

\footnotetext{
${ }^{38} \mathrm{Te}$ wheke is another useful Māori health model. However, I do not detail it here, as
} I am still learning about this with the Māori advisor. It is a more complex model. 
they are more general aims. How the targets are measured is unspecified and not fixed, which accommodates variability and student preferences. Although these targets are still measured in some way, how they are measured can change and develop. A similar approach I've encountered is narrative assessment, where objectives don't need to be quantifiable. Narrative assessments involve writing and analysing learning stories that capture progress in student's learning. They record subtle interactions between students, their peers and learning environment. This approach also allows more flexibility in how success is measured. However, the MoE suggests that measurements should be in relation to national standards (Ministry of Education, 2009b).

Small's (1998) musicking offers passive listening a more equal position with that of active participation. All forms of participation (passive and active listening) are valid in musicking and therefore can be considered as successful session outcomes (Rickson \& McFerran, 2014). Elliott's (1995) musicing shares with Small's construct the idea that music is an activity rather than a thing. It acknowledges the social and cultural contexts in which the interaction, both active and passive, takes place (Aigen, 2014). I would also add the need to have times where support doesn't involve musicking/musicing, where we support in silence and with our presence. 


\section{4. "Truth" 3: Success is predictable and therefore can be planned}

\subsection{Journal entry}

What were his goals? Did he reach them? Any new aims? IEP after IEP asking the same thing. I answer robotically; I know the lingo, the 'appropriate' way to describe and "plan" for success in developmental terms. But for some children we are stuffing square pegs into round holes.

In the culture of the well and able, goals and plans can lead to magnificent places. Places made of dreams and Kodak moments. There is an underpinning of certainty comforting you with its predictable lullaby. If you work hard you can get to the place you envision. Sipping on a celebratory wine when you finally reach your intended destination: the taste of future aspirations on your tongue.

For some, in the culture of the ill and disabled, each day is not like the one past. Every hour changing like the winds on Makara hill. There is no calm rock of predictable and certainty not granted. Goals morphing like play dough each minute that passes. The skill of improvising is mandatory as plans are no good here. Sipping on wine spontaneously when life offers a nice surprise, tasting the beauty of the here and now.

It is different this way of life. Joy snatched in a gobble of ice cream, in a shared joke, in the sea breeze tumbling through your hair. Presently living provides the needed strength to keep swimming. To feel the repeated disappointment in a goal not reached, the grief in the dream lost, the guilt that you did not live up to an expectation, the anger that for others it looks easy, all rub salt on a weepy wound. Are the goals we predict for others really what they want?

When I entered Matilda's classroom, her teacher tilted his head in Matilda's direction with a, 'it's one of those days' look. She was wedged between the wall 
and the bookshelf with pieces of white tissue sprinkled over her: A cozy safe place where she could disperse sadness with each tear of tissue made. She looked up and said, "Sad face, no music". "OK" I said, and I sat beside her for five minutes.

"Music now?" I asked, she responded by putting her shoes on. Usually she comes with some buddies, but today it was not appropriate. She chose to play with the boomwhackers in the hall. On "ready steady go" we rolled one each to see whose would go the greatest distance. She listened carefully to the sound of the boomwhackers rolling along the hard surface, resonating in the big empty space. "More" she asked excitedly as she chased after them. On the next turn our tubes crashed and she laughed. On returning to class she was calm and ready to learn as her immediate need was met, while her formal planned goals were abandoned.

So how do I articulate what my ${ }^{39}$ future 'goals' are for those who we are meeting for? Just like the conditions they have, I want them to be malleable: changing in a heartbeat to keep the swimmer afloat. How do I translate that into this IEP format of planned and predictable goals set?

\subsection{Truth}

The fact that SMART goals can be set before success occurs relies on the underlying belief that success can be predictable, and we can plan for it. In IEP's I have been involved in SMART goals are made by the professionals, who make a plan for success. Other processes too, such as planning and assessment meetings, rest on the truth that there is a way to plan for success and that professionals can predict how it will occur. If we do plan $\mathrm{A}$ and $\mathrm{B}$, we will get the result of $\mathrm{C}$.

${ }^{39}$ Again we can see the presence of the discourse of expertise in this journal entry, as I try to articulate "my" future goals for this person. This was discussed earlier in chapter 4 . 
This discourse of predictability is sustained by Evidence-Based Practice (EBP). EBP made its way into therapy and education through evidence based medicine (Aigen, 2015). Music therapists such as Taylor and Thaut have medical based practices congruent with EBP principles (Aigen, 2014). In EBP there is a hierarchy of evidence that determines the predictability of the therapy outcomes. Meta-analysis and randomised control samples are at the top, descriptive and qualitative studies lower down, and opinions at the bottom. Accordingly, some types of evidence are believed to predict certain outcomes/success better than others.

In response to ongoing demand from educational bodies, music therapists have increasingly needed to work within and provide EBP. There have been calls to advance MT through EBP from the American Music Therapy Association (Abrams, 2010). Models, such as The Rational-Scientific Mediating Model, have been developed to help "predict outcomes" and interpret findings in MT research (De l'Etoile, Dachinger, \& Lathroum, 2012). Worksheets, such as the Therapeutic Function of Music Plan are thought to support predictable outcomes (HansonAbromeit, 2015). Kern (2011) has outlined a EBP decision making process. Within a NZ context, EBP has made its way to the professional body. In reading through documents for Music Therapy New Zealand's new website, EBP was mentioned as framing practice. Aigen (2015) notes that, "the current conventional wisdom appears to be that the drive toward EBP is the single most important external force influencing MT and music therapists must accommodate to these demands or face irrelevance" (Aigen, 2015, p. 14). He believes the foundations of EBP's are flawed. Despite the flaws of $\mathrm{EBP}^{40}$, it is promoted as a predictable way to generate success in education.

\subsection{Power}

I found that educations focus on predictable and planned success can restrict MT practice:

- Discourses of predictability provide certainty at the expense of flexibility and the client in the here and now.

- Discourses of predictability produce practices that can control at the expense of working in a state of unknowing.

\footnotetext{
${ }^{40}$ Discussed further below.
} 
- EBP de-emphasises the importance of therapeutic relationships in shaping encounters and decision making (Eisenberg, 2012).

- It encourages therapy services to adhere to EBP for financial gain.

- The EBP hierarchy of evidence suppresses the client's voice as their subjective experiences/opinion of therapy is on the lowest level of the hierarchy (Aigen, 2015).

Kenny (2006) warns that the danger of the illusion of predictability in society is that all behaviours, including death, can and should be predictable (p.19). This predictability provides certainty at the expense of flexibility and may lose or reject the client in the here and now in favour of a future self/outcome. Predictability reduces the need for services to be responsive as plans are followed and stuck to. Supporting a more outcome driven not process-orientated approach. Predicted future outcomes may hinder a person from seeing unexpected and unplanned successes, as planning influences what one looks for in the work.

When one family I worked with let go of the plan for their child to talk, they noticed things that their child was doing that they previously didn't comment on.

Predictability discourse requires us to be in control of what will eventuate. It doesn't foster the capacity of tolerating uncomfortable feelings in MT work or to work in a state of unknowing. Without spontaneity we cannot discover surprises (Kenny, 2015). It may be difficult to accept the unknown unknowable in others and the possibilities it creates (Roman, 2016).

The suppression of spontaneity in predictability discourse has consequences for working with music in therapy. Music making, like improvisation, is an unpredictable activity. One does not know how it will go and what it will sound like. We cannot predetermine exactly how music will affect us. Consequently, outcomes of music making with another person are unpredictable and cannot meet the demands of the predictability. Therefore, the place of free music-making in generating success for students becomes questionable within this truth. Structured or pre-composed music played as notated, would be preferred in this discourse in order to predetermine success. However, this isn't the way I practice MT. Ironically, it is exactly music's 
unpredictable nature that has had value for many students and families I have worked with.

Similarly, as relationships themselves are unpredictable, the relational aspect of therapy may become de-emphasised over the predictable interventions that the therapist employs. The therapeutic relationship cannot be validated due to its individual variability. Whilst it is fundamental to the therapy, it is unclear of how EBP can be implemented meaningfully to accommodate relational aspects (Aigen, 2015).

In a culture that loves the idea that the body and mind can be controlled, those who cannot control their bodies or minds, and those therapies who are supposed to have helped control them, are positioned as failures (Nabors \& Pettee, 2003; Wendell, 1997). This position impacts on financial resource allocation. The 2017 budget is based on the idea that if we intervene early, disabilities will be cured or not impact as greatly on societal resources. Funding was taken from students with disabilities in later schooling years to fund early intervention (services for children aged 0-5). This was done under the guise that early intervention can predict the success of significantly decreasing disabilities and the need for support later in life. They explained that there will be a "better chance of putting them onto positive and sustainable achievement paths" (Kaye, 2017, p. 1). This fails to recognise the ongoing nature of disability, and that not all bodies/minds can be controlled. The type of success planned for is one of "cure" instead of successfully providing better ongoing support that accepts and meets the needs of diverse ways of being.

EBP is believed to be a predictor of money best spent, forecasting neoliberal success. The MoE divvies funds to services based on EBP. The 2012 budget provided funding to implement further evidence-based behaviour programmes (Ministry of Education, 2012). More recently, the 2017 budget increased funding for the Incredible Years programme, $\mathrm{ABC}$ and beyond programme, early intervention and severe behaviour services, as they were claimed to be evidence-based programmes. This budget is based on the premise that we can predict that funding evidence-based practices early in children's lives will mean less support needed for such children later in life (the measure of success). 
Due to this, programmes may develop and adapt their approaches to meet EBP principles for funding. It is financially in their best interests to do so regardless of whether this best serves the interests of the people they are working with. In a 2017 budget announcement it was stated: "Funding for all of these programmes has been made conditional on having robust evaluations in place, so that we can know that we are getting value for your money" (Kaye, 2017, p. 1). These evaluations are framed with the evidence hierarchy. Consequently, disabled people's voices may once again be lost as their opinions are on the bottom of the evidence hierarchy.

\subsection{Ethical considerations}

The principle ethical dilemma of predictability in education is its restriction on the work conducted with people with disabilities. The disabled body/mind challenges the ethical search for certainty. The unpredictable, unstable, disabled body/mind is minimised and suppressed through planning processes which limit responsiveness to bodily/mind changes and restricts the potential generated from music therapies more flexible unpredictable tools. It raises the ethical question: what is the role and value of unpredictability and uncertainty in successful outcomes?

Fostering an attitude of negative capability and centering in the present moment may assist in countering some of the power effects of discourses of predictability on success. Carel (2013), who experienced chronic illness, explains that, "by focusing on the present, I learnt to discount the future, while it seemed to me that so many of my friends were doing the opposite" (p.144). I agree with Carel (2013) that, for me, one way of living well with illness is by living in the present. The present is the only thing we may have, the future is unpredictable and uncertain.

When working with music and methods such as improvisation, it is necessary to have a willingness to play with uncertainty and contradiction (Levine, 2013). John Keats notes, in a letter to his brother, that a fundamental aspect of the aesthetic attitude is: “... Negative Capability, that is, when a man is capable of being in uncertainties, mysteries, doubts, without any irritable reaching after fact \& reason" (Keats, 1899, p. 277). This is a space I enter as a music therapist, and one that has great value to the process, even though it struggles to be validated. Potential outcomes aren't clear and distinct and cannot be precisely predicted and produced beyond any doubt. Kenny (2006) illuminates that there is a certain level of mystery and magic in MT. She states 
"uncertainty betrays outcome or product and leaves the space for surprises in the MT experience" (Kenny, 2015, p. 464). The present focus of the music occurring in the moment is necessary for growth.

I also found it helpful to draw on discourses of palliative care within education, as it recognises that the body is constantly changing, not stable, and cannot be controlled. This provides a framework for valuing the present self and supporting different experiences of being: being heard, spiritual experience, aesthetic experience, experience of trust, experience of play. Kenny's (2014) field of play is also helpful here as she describes being as an essential aspect. This MT approach acknowledges the concern for quality of life (like that of palliative care).

I note that the meaning of "palliative" in the New Collins Concise English Dictionary (1982) simply means to "relieve without curing" and that to palliate is to lessen the severity of the pain without curing: alleviate. There is nothing about dying. In NZ, the medical profession and the hospice movement have adopted palliative to apply to care specific for the terminally ill. This meaning is also applied to the funding criteria set by the Ministry of Health (Swenson, 2012). However, like Swenson (2012), I have found it helpful to think about Palliative Care Plans for the non-terminally ill. A nurturing of social, emotional, spiritual, physical being to create the optimal quality of life for those who live with permanent conditions that they will not recover or die from. These plans do not predict what is going to happen. They constantly explore what is possible in the present moment. 


\section{Chapter 8}

\section{Creating the Self as a Work of Art (Part One): Developing Post-Ableist Music Therapy}

“Creating oneself": Photograph of painting activity with a toddler

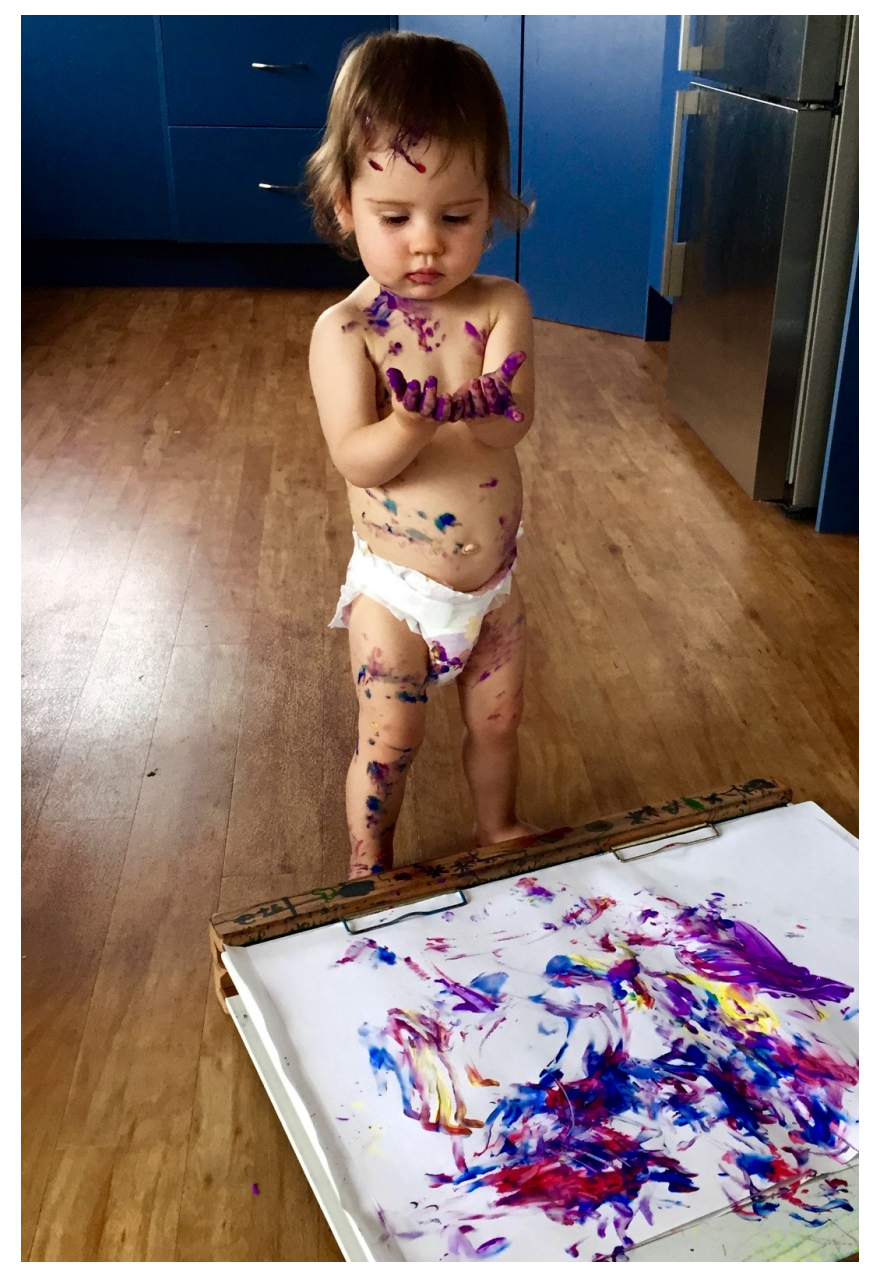

[Image of a toddler looking at paint on her hands. Her painting is in the foreground] 


\section{Introduction: Searching for a way to work without a vegetarian having to eat meat.}

The prior analysis chapters have primarily looked at the first two domains of Foucault's critical ontology of ourselves, truth and power, within my earlier practice and the contexts I inhabited as a practitioner and $\mathrm{PhD}$ student. They also touched on the third axis-ethics-by identifying ethical considerations and moments throughout. This chapter further develops the ethical domain. It does so though the proposed Foucauldian tool of creating the self as a work of art, which is part of the ethical care of the self. Engaging in creative process is a way to defamiliarise and reconceptualise practice.

Although my therapy work was considered by others to exhibit important qualities, such as being enthusiastic, empathetic and relational ${ }^{41}$, I felt there was more to be done. I began to actively address the ethical considerations and power effects previously outlined as my research progressed. Consequently, my practice changed considerably alongside this research. This chapter explores those changes, suggests further steps and develops the research findings.

To date (2017), the ethical care of the self (my actions) has taken an ecological approach. That is, my actions have not only been directed towards my own practice but also towards the broader contexts that my practice takes place in. Ethical care actions within my practice:

- I have increased collaboration in my paperwork, much like the practice of narrative therapy. Students are included in the process of writing reports/assessments, co-writing where possible (appendix 2). Recordings of their music have sometimes been included. For students who do not communicate through spoken or written language, I have incorporated their unique ways of communicating. One student made a collage from photos of our sessions together. It is important that normalised ideals are not privileged, and that people's unique ways of contributing are valued in collaborations.

\footnotetext{
${ }^{41}$ Evidenced in emails from schools and feedback from colleagues.
} 
- Whether or not the person identifies as Disabled is included in assessments.

- I increased the acknowledgement of my own subjectivity in discussions and in written records.

- I attempted to reduce the focus on goals. Some contexts still require goals, which is a constant process of negotiation. I instead focus on exploring and privileging the possibilities that emerge in the process. In reports I use the heading "areas of exploration" instead of "goals".

- I changed the language I use, which opened up new possibilities (Drewery \& Monk, 1994, p. 309). I am more aware of language that dehumanises/deauthorises. I prefer to avoid pathological terms and describe behaviour and actions with descriptions that support understanding. For example, using, "self-regulating" instead of "stimming" or using "way of coping" instead of "defensive strategy", or using "enthusiasms" instead of “obsessions”, where appropriate ${ }^{42}$. I don't refer to people as cases and question disability metaphors I use.

Ethical care actions towards wider music therapy (MT) contexts:

- With the help of Claire Molyneux, I made suggestions to the NZ Standards of Practice Working Party for the standards of practice and code of ethics documents to be more inclusive of different disability perspectives (appendix 3).

- I did a presentation about disability issues in MT practice at a NZ Hui (2016) and advocated for acknowledging illness/disability within the MT profession. I note that the NZ symposium following this presentation included, for the first time, disability access questions, such as "Do you require a disabled park?" on the registration form. The MT professional body has been responsive in thinking about inclusion.

- I wrote to the registration board about raising the importance of self-care during the practicing certificate renewal process in order to balance the

\footnotetext{
${ }^{42}$ I acknowledge that there may be some situations/contexts where "enthusiasms" may not seem appropriate. For example, in an acute mental health setting where someone is not feeling "enthusiastic" about their obsessive behaviours. However, like Prizant (2015) I have used enthusiasms in relation to some people with Autism Spectrum Condition where intense interests can be a helpful resource.
} 
message that working hours and hours of professional development are the only things that create competence. ${ }^{43}$

Even with all the changes and actions above, like Kenny (2015) described, I still felt the "grinding and irritating feeling" (p.465) in my gut about how to describe my practice. I could not sit comfortably. I had un-become. I was wordless. I had yet to find something that fitted as comfortably as Cinderella's slipper. I had been trying on different shoes for years and couldn't find the right one. They were just a little too tight for me to walk straight and proud. Awkwardly I limped.

"What are you searching for?" a supervisor asked. "I am searching for a way to do my job without a vegetarian having to eat meat" I replied. Lee (2003) lamented that music-therapy speak alienated him from his colleagues. I felt the same, for different reasons. I realised that I had to redefine it in my own way (Kenny, 2015). However, there was much difficulty in doing so. My experience, work and the theories I explored were often at odds with each other (Halifax, 2009). There was not one theory that would fit: each threw certain patterns into relief and others into shadow (Halifax, 2009). I needed a patchwork blanket of theories that would cover and address the ethical concerns raised in the analysis chapters. Examples raised in the analysis chapters can be seen in the patchwork blanket below ${ }^{44}$.

\section{Figure 1: Ethical concerns derived from dominant discourses in the practice studied and some counter discourses}

43 This issue came to my attention in the chapter 7.

${ }^{44}$ I am aware that other theories may address these issues, but I have noted orientations/theories that I drew on during the analysis and at this stage in the process. 


\begin{tabular}{|c|c|c|}
\hline $\begin{array}{l}\text { Addresses binary thinking } \\
\text { that contributes to the } \\
\text { dividing practices of } \\
\text { disabled people } \\
\text { - Indigenous health } \\
\text { models } \\
\text { - Narrative therapy } \\
\text { - Field of play } \\
\text { - Posthuman } \\
\text { approaches }\end{array}$ & $\begin{array}{l}\text { Support the interdependence } \\
\text { and interconnection of } \\
\text { human and non-human } \\
\text { entities: addressing } \\
\text { anthropocentrism } \\
\text { - Ecological models } \\
\text { - Posthuman } \\
\text { approaches } \\
\text { - Field of play } \\
\text { - Te Whare Tapa } \\
\text { Whā }\end{array}$ & $\begin{array}{l}\text { Addresses the dominance of } \\
\text { humanistic thinking that } \\
\text { exclude some disabled ways } \\
\text { of being } \\
\text { - } \quad \text { Posthuman } \\
\text { approaches } \\
\text { - Narrative } \\
\text { approaches }\end{array}$ \\
\hline $\begin{array}{l}\text { Addresses standardisation } \\
\text { and increase respect for } \\
\text { different ways of being and } \\
\text { human variation (plurality). } \\
\text { - } \quad \text { Posthuman } \\
\text { approaches } \\
\text { - Queer music } \\
\text { therapy } \\
\text { - Narrative } \\
\text { approaches }\end{array}$ & $\begin{array}{l}\text { Addresses the subjugation } \\
\text { of immeasurable aspects } \\
\text { (stillness, beauty, creativity, } \\
\text { aesthetics) } \\
\text { - Aesthetic MT } \\
\text { - Field of play } \\
\text { - Music centred } \\
\text { approaches }\end{array}$ & $\begin{array}{l}\text { Addresses the need to value } \\
\text { the role of unpredictability, } \\
\text { surprise, disruptions, } \\
\text { unknowing } \\
\text { - Aesthetic MT } \\
\text { - Music centred } \\
\text { approaches } \\
\text { - Field of play } \\
\text { - Psychodynamic MT }\end{array}$ \\
\hline
\end{tabular}

To accommodate and address these needs, such a practice would be based on the primary ethic of an increased relationality that encompasses:

- Subjectivity

- Mutuality (non-hierarchical being, partnership)

- Interdependence-unconditional relationships of responsibility

- Questioning/Negative capability

- Agonism $^{45}$

- Creativity and beauty

- Diversity

Like many well-intentioned relational approaches, my analysed previous practice often talked the language of relationships, but was still confined in some ways to the

\footnotetext{
${ }^{45}$ Foucault spoke of an "agonism" in which there is reciprocal incitation and struggle; which isn't a confrontation that paralyses both sides but a productive permanent provocation of power (Foucault, 1982).
} 
individual (Sampson, 2003). For example, individualising therapeutic concerns and not acknowledging the role of non-human agents, limited its relationality. An extended form of relationality was needed in my practice. Relational ethics insist that relationships are key to human survival and well-being and that relationships are systems that cannot be reduced to the processes of its parts (Sampson, 2003). A relational paradigm has slowly but surely overtaken self-contained individualistic views that were a large part of therapeutic practices (Sampson, 2003) and there are still ways to further extend this in practice.

The rest of this chapter explores how I have redefined my practice with this ethic in mind. It extends the work outlined in this introduction and has important findings for all of the research questions. The chapter will be structured in the following way:

- "Starting with the human: from human to posthuman": this explores the basic unit of reference for the human and describes my shift away from humanism to posthumanism.

- "Person meets music: the musical posthuman": this section includes three musical improvisations, which were creatively reflected on to gain clarity and further direction: A creative musical journey towards the development of PostAbleist Music Therapy (PAMT).

- "Putting it all together: Post-Ableist Music Therapy": descriptions of PAMT and ableism are given as well as vignettes demonstrating aspects of PAMT.

This chapter is part one of the two-part exploration of creating the self as a work of art. 


\section{Starting with the person: From human to posthuman}

I have to start somewhere in redefining my practice ${ }^{46}$. Or rather restart again.

Redoing. Reworking. Reinventing. I go back to the beginning, "What determines our conceptions of and ways of doing MT are our basic views on the nature of the individual, or the human being" (Ruud, 2010). What is my idea of the human person? From asking this, I recognise the foundation of humanism that much of MT rests on. Many of the MT approaches stated in figure one are keenly described as humanistic or describe humanistic ideals. As Ansdell \& Stige (2018) contend, the logic of humanism seems to be solidly present in contemporary MT. It has been embraced by a number of leading MT theorists (Aigen, 2014).

However, I need to rethink humanism as it limits the relational ethic of my practice with its focus on independence and the centrality of human agents. Sampson (2003) suggests that in order to have a genuinely relational practice it must be based on unconditional relationships of responsibility to others. In order to do so, there needs to be a shift from the humanistic character that dominates - the self-contained, autonomous self. I look to rebuild these foundations to support an extended relational ethic of practice with aspects of Braidotti's posthumanism (2013).

Her first paragraph grabs my attention:

"Not all of us can say, with any degree of certainty, that we have always been human, or that we are only that. Some of us are not even considered fully human now, let alone at previous moments of Western social, political and scientific history. Not if by 'human' we mean that creature familiar to us from the Enlightenment and its 'legacy"' (p.1).

She offers me a way to re-think the basic unit of reference for the human: posthuman subjectivity is an interconnected interdependent relational entity that is embodied and embedded. The posthuman subject forms an "enlarged sense of interconnection between self and others, including the non-human or 'earth' others, by removing the obstacle of self-centred individualism" (Braidotti, 2013, p. 190). Renewing claim to

\footnotetext{
${ }^{46}$ I have switched to writing in the present tense to give the reader a sense of journeying with me in the creative process.
} 
the earth, community, global community and belonging by singular subjects through a strong sense of collectivity: fostering collaborative morality. The posthuman subject is a non-hierarchical creative being with multiple capacities for relations of all sorts and "modes of communication by codes that transcend the linguistic sign by exceeding it in many directions" (Braidotti, 2013, p. 190). Allowing room for communication as an "embodied and impure activity based on noise, relationality, and reciprocity" (St. Pierre, 2015, p. 330) and technological voices for example through iPad programmes.

I can walk a little easier with this posthuman subject: In the figurative sense at least. In the literal sense, I am unwell again: My legs have become heavy and hard to move at times. I myself am becoming posthuman ${ }^{47}$ as my rational thought, linguistic prowess and independence gives way to something else. My legs become my husbands. He holds my weight, moving me to the other room. The blood pressure monitor becomes another limb that informs and supports us to know when to get moving again. I feel too much to ration myself out of tears. Words, w o r d s ....w h a $\mathrm{t} w$ a $s \mathrm{t}$ a $\mathrm{t} w$ o $\mathrm{r}$.... I wave my hand to his question as my brain empties the alphabet. I develop my posthuman codes of grunts and particular looks. The posthuman is disabled, interdependent with non-human and human agents, created and relieved by both. I need to become posthuman for if I stay human on this earth, I will continue with much struggle. I realise I am jumping all over the page now. This is how life currently feels. Disrupted. In short snippets.

\section{Person meets music: The musical posthuman}

Between body disruptions, I am in PhD supervision struggling to articulate a new practice. The suggestion is made to "creatively play with music" instead. It feels like the right (and only) thing to do at this juncture. Music can provide release and relief from words: to make music is to engage in a form that is not necessarily narrative driven (McCaffrey \& Edwards, 2015). It can support research by unlocking alternative ways of thinking through its facilitation of playing in the unknown (Hoskyns, 2017). This differs to narrative therapy, which is verbally orientated.

\footnotetext{
${ }^{47}$ We cannot say with certainty that we have become posthuman or that we are only that - it is a process-oriented vision of the subject (Braidotti, 2013).
} 
Many of the theories informing me while creating myself endorse the role of creativity. One of the stated criteria in posthuman ethics is making a "new link between theory and practice" that includes the central role of creativity (Braidotti, 2013, p. 191). This correlates with Lee's (2003) reminder that music therapists cannot forget to make links with the practices of music. Aesthetic MT and music-centered approaches are based on this principle. Furthermore, "faith in the creative powers of the imagination is an integral part of feminists' appraisal of lived embodied experience and the bodily roots of subjectivity" (Braidotti, 2013, p. 191). With such endorsement, my next step is to make music. To create music is to be open to all possibilities (Lee, 2003). The outcome is uncertain. This uncertainty parallels my experience of illness.

The music described below was a joint activity that belonged to none of us, rather it was a co-production of being together. Therefore, with a relational ethic, I alone did not develop the findings to come.

\subsection{First Improvisation: "The failure of 10 minutes and $\mathbf{4 0}$ seconds"}

At my next supervision meeting with Sue and Sarah, I asked if we could make some improvised music together. A collection of instruments was placed on the table for a free improvisation.

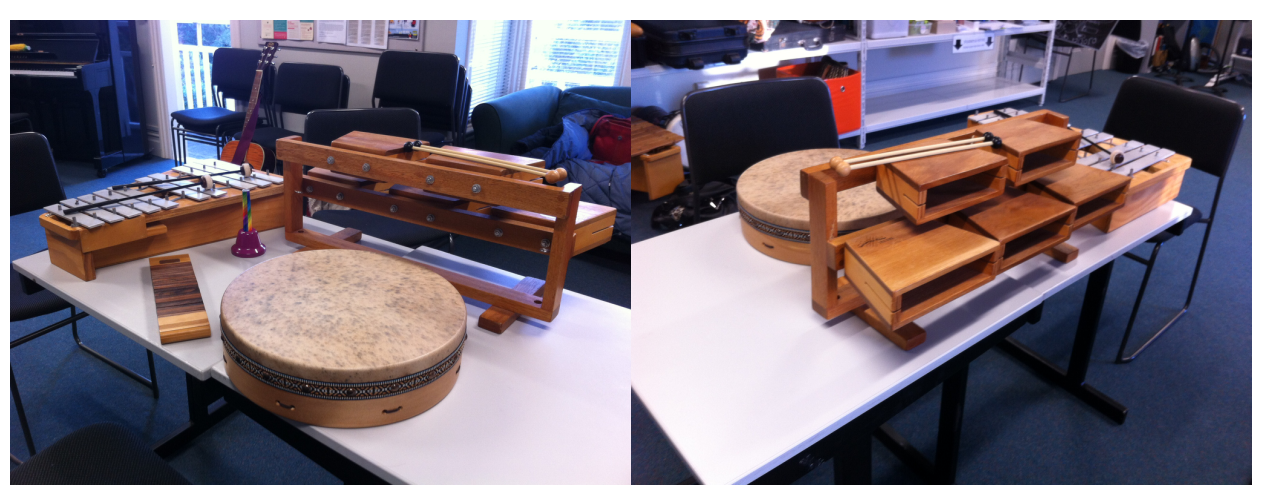

[Two photos of tuned and un-tuned percussion on a table]

After this improvisation a discussion took place with my supervisors, which was audio recorded. The discussion was transcribed, important ideas were highlighted, then a creative writing piece was written. What emerged was an improvisation that further explored ideas around success. Therefore, I creatively revisited chapter 7, which became part of my reflexive process. 


\subsubsection{Creative writing piece: "The failure of 10 minutes and 40 seconds"}

For this improvisation I said, "No play rules": It was never meant to be about anything. Sarah suggested the elephant feet (temple blocks) ${ }^{48}$. I told her of my previous failures with them. I played them anyway: I had a hope that I may enjoy them this time around.

We heard the clonk, clonk, clank of the elephant feet.

Breaking my hopeful expectations.

"Your parts were lovely, I just found it hard to match in the way that I wanted to: to be a part of things in the way that I wanted", I said.

I spent my time trying to escape playing it.

We heard the clonk, clonk, clank of the elephant feet.

Its heaviness broken with raucous laughter.

Sarah's permission to be silly bubbled up "something irreverent". The impersonation of moreporks and oyster catchers: The playfulness,

provided another way to escape from playing it.

We heard the clonk, clonk, clank of the elephant feet.

Its dominant physical structure alluring its player.

Sue said, "It's there, in your face like you're supposed to use that...But you actually wanted something around the corner"-yes, that was where I found the shaker.

Playing the shaker was another way to escape from playing it.

${ }^{48}$ The temple blocks had been called the elephant feet in my practice for many years by students. 
We heard the clonk, clonk, clank of the elephant feet.

With its five notes shaped in boxes like the parameters of success.

"You will tick this box, and you will tick this box, and if you can't tick that box you haven't succeeded” Sue reflected: I hit one 'box' hard with tremolo.

It was another way to escape from properly playing it.

We heard the clonk, clonk, clank of the elephant feet.

That is, until we started to "shush" the sounds in the room.

My voice rebelling against this dominant instrument: I would not stop shushing. The shushing grew louder until there was nothing more to play. With relief I laughed.

Using my voice was a way that I could escape from playing it.

We heard the clonk, clonk, clank of the elephant feet.

Its barrier tall and wide, obstructing my view.

Behind the wall, isolating. Afterwards Sarah decided to "clear the decks" and removed the elephant feet: I could see everyone.

If it wasn't there, I did not have to escape.

I heard the clonk, clonk, clank of the elephant feet.

Its music was different to the music I privilege in knowing: the therapeutic music that connects and moves us.

I heard the symphony of how music can fail: That is, the music that can create disconnecting barriers and obstructions.

I want to escape from playing that. 
I heard the clonk, clonk, clank of the elephant feet.

And now I no longer hope to enjoy playing it.

There are so many more instruments to choose:

Exciting possibilities to play.

\subsubsection{Key finding from "The failure of 10 minutes and 40 seconds"}

During the improvisation, discussion, and creative writing, the elephant feet became a symbolic representation of disabling barriers. From this creative exercise, I extrapolated that a key indicator of MT success is the removal of barriers that limit success: including how success is framed and what it can be.

\subsection{Second improvisation: "The third"}

At the next supervision meeting another improvisation occurred. This time I chose two pianos for us to play (small shakers and bells were also available). No play rules or preconceived ideas of what to think about when playing were given (audio 1 example: https://soundcloud.com/user-419330485/sets/audio-1-example-excerptfrom-2nd-improvisation $)^{49}$.

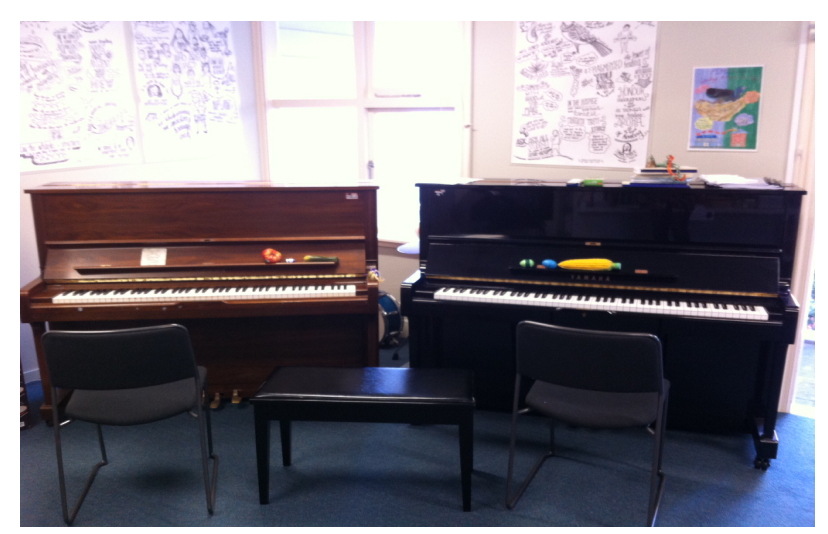

[Two pianos side by side]

After this improvisation a discussion took place with my supervisors, which was

\footnotetext{
${ }^{49}$ Audio examples can be listened to by copying the web address into your browser or clicking the link.
} 
partly recorded (the recording device stopped part way through). I used the transcription from the recorded section and my notes to highlight interesting ideas that emerged. What emerged was an improvisation and discussion that further explored ideas around dichotomies (a creative revisiting of chapter 6).

\subsubsection{Creative writing piece: "The third"}

When they started, they did not know of sides, or camps, or positions.

They stood randomly while the bells rang.

Resonating together.

One picked one piano and then another picked the other.

The third sat in the only seat left.

The one between them.

The two on separate pianos started a dialogue, or was it a debate?

From the two solid piano frames.

Toing and froing.

The third did not know which keys to play and when.

Scared of notes in the wrong place.

Or joining the wrong piano.

With this ambivalence, she randomly tested out different notes.

Surprising the others with unpredictable tone and rhythm.

She changed the direction.

Falling between the two pianos she sometimes escaped the heavy frames.

Calling the two away from their pianos as she played the bells.

But they kept playing... 
Except their playing moulded into tender moments of togetherness.

One supporting the other piano's melodies.

Talking with, not to or over each other.

Perhaps unknowingly, the third kept finding "the unheard music"50.

She pressed two piano keys that lay side by side.

The others took turns to copy.

There was silence between the two different notes singing together.

Space to breathe and to be undone by the clashing.

Beautiful dissonance.

When they finished, they did not know of only their side, or camp, or position.

They knew of the third's ability to connect them and of her beautiful différance ${ }^{51}$.

\subsubsection{Key finding from "the third"}

From the improvisation, discussion and creative writing process, I discovered that a key role of the music therapist is to be like The Third ${ }^{52}$ : to find ways music can both facilitate moments of connection and find new spaces that differ from existing structures or spaces that are less restrictive (new/less restrictive spaces). Importantly, the descriptions of the third suggests an agonistic state where the productive possibilities of playing in the tensions between different structures was illuminated. I expand on this idea later in the chapter.

\footnotetext{
${ }^{50}$ Elaine Streeter, a UK music therapist and researcher, used to teach students to listen to the "unheard music" when learning to improvise (Guildhall School of Music and Drama).

${ }^{51}$ I am making reference to Derrida's idea of différance here, see chapter 6

${ }^{52}$ I also acknowledge that this links with Jessica Benjamin's intersubjective "thirdness". She contends that the degree with which we ever manage to grasp twoway directionality is only from the place of the third, a vantage point outside the two.
} 


\subsection{Third improvisation: "What is this?"}

The final improvisation took place at $\mathrm{PhD}$ supervision a month later. This time a range of drums were selected. This improvisation differed in that we were aware that there were two remaining main areas of the analysis that we hadn't explored musically-disclosure (chapter 5) and invisibility (chapter 4). Before playing, we posed the question: "Is this disclosure or is it invisibility"? Apart from considering this question, no other play rules were given.

After this improvisation a discussion took place, which was recorded and transcribed. I listened to the improvisation again and used the transcription to note interesting ideas that emerged. What unfolded was a creative process that explored the interconnectedness of invisibility and disclosure. You can't have a disclosure without invisibility; it explored both.

Because I became stuck and struggled to start writing this piece, I used the free writing technique, where I set a 20-minute limit. Within that time, I had to constantly write, drawing on what first came to mind. Due to this, the writing is perhaps less worked/refined.

\subsubsection{Creative writing piece: "What is this?"}

A sound $=$ a disclosure

A silence $=$ an invisibility

If only it were that easy.

Loudly banging while invisible.

Playing a rhythm that covers you.

A visible silence.

How do I know what's what?

I try to find an answer from our drums.

Some disclosures were forced.

The surface of one was frantically rubbed raw in the hope to break through to the middle: to intrusively know what lies beneath skin deep. The question was asked, "Whose rules?" Tell them what they want to know. Tell them your personal 
intimate details ${ }^{53}$. There is a demand before a disclosure. Some demands seem reasonable and others just plain silly: "I have been wanting you to tell me about your glasses", she says: A funny joke. "I need you to tell me how your illness impairs your ability to function": no one laughs ${ }^{54}$. What needs to change to make the latter seem funny to ask? This drum took a constant beating to educate others.

On another drum the snake was awoken. Sneakily betraying, letting something slip. “The music pulled it out of me," she said, "I couldn't stop it". Not wanting to disclose but the snake came out: To be pointed out, to be put out, and to be outed. Outside there are snakes. It's not always safe. It is best to hide and wait for the environment to be safe.

Some disclosures were self-driven.

Enough of the surface scratches, the beating pattern of one drum became deliberately arrhythmic. It resisted the entrainment from others: Loudly proclaiming its difference in celebration. Liberated. Beating in the face of normality: challenging its formation. The other drums were interrupted from their trance like regular beating haze. Falling off the measure, their drumming changed.

Invisibility is a point of reference.

The players look at the drums from the same position, looking down at their skins. The resonant chamber of the body and opening remained invisible. Other angles unexplored. If it were seen upside down there would be no need to disclose its "hidden" melodies. No "hidden" music or disclosures. The playing would simply be different.

A sound can be silencing

A silence can speak resistance

An interruption can be celebrated

The upside down can be a better way up.

${ }^{53}$ Whilst writing this, an Australian disabled blogger, Carley Findlay, was asked live on ABC Radio if she can have sex (Carmody, 2018). Disabled people are often demanded to disclose such personal information.

${ }^{54}$ This question is often asked in order to acquire accommodations at university and school, during applications for financial support, and asked informally. 
From the improvisation, discussion and creative writing process, two important ways of managing invisibility and disclosure in MT stuck out for me: 1) Creating less disabling environments (within sessions and professional practices) reduce some of the issues around forced intrusive disclosures that ableist environments demand. I recognise from the writing above that forced disclosures differ to claiming a disabled identity for the self that resists normativity and challenges environments to become less disabling. Therefore, creating less disabling environments may be created through both the reduction of forced disclosure of personal details regarding one's body/mind and self-driven disclosures of disabled identities (if one identifies this way). 2) Developing the ability constantly to question your frame of reference can alter what you hear and see, changing the need for disclosure and changing the visibility of hidden aspects.

\section{Putting it all together: Post-Ableist Music Therapy (PAMT)}

I found the improvisation, discussion, and creative writing process helpful as a way of gaining clarity amongst the mass of qualitative data. It was also helpful in creating direction and moving me outside of the dominant discourses of the practice studied. During this process, five main aspects of practice emerged as important focus areas. These were (in order of appearance):

1. The removal of barriers

2. Supporting moments of connection

3. Finding new/less restrictive spaces to be in practice

4. Creating an environment and experience that is less disabling

5. Questioning my frame of reference

Reflecting on this list, I see that they are all working towards the same agenda: to "create an environment and experience that is less disabling" (number 4), through the removal of barriers (number 1), supporting moments of connection (number 2), finding new/less restrictive spaces to be (number 3), and the questioning my frame of reference (number 5). Looking back at the ethical concerns and issues highlighted in the introduction, it appears that they too are accommodated within this practice focus. To create an ethic of increased relationality through subjectivity, mutuality/nonhierarchical being, interdependence, questioning/negative capability, agonism, 
creativity/beauty, and diversity, would support the creation of a less disabling environment in practice. The focus areas above, therefore, rely on an increased relational ethic, as barriers, disconnections, constricting spaces and normative frames are constructed through a limited humanistic relationality based on the able bodied/minded.

\subsection{Post-Ableist Music Therapy Definition}

To hold central to practice the creation of a less disabling environment/experience, through focus on the aforementioned areas and enhanced relational ethic, led me to the development of what I call, and define as, Post-Ableist Music Therapy (PAMT) ${ }^{55}$. PAMT is a creative process that seeks to work with a person and community to provide an environment and experience that is less disabling through addressing barriers, exploring connections, and providing new/less restrictive spaces through primarily musical or music related experiences. It welcomes different ways of being and resists a one-size fits all approach. Instead, the client and contexts guide the therapist. In this way, the definition I give of PAMT cannot be too prescriptive. It is necessary that the therapist questions their frame of reference during the process to unearth and undo any ableist assumptions. This is important because "We are all, regardless of our subject positions, shaped and formed by the politics of ableism" (Campbell, 2008, p. 151). PAMT is primarily based on aspects of posthumanism to counter the ableism and limited relationality inherent in humanism. As the posthuman subject is an interconnected interdependent relational entity it counters attempts to make the person whole or hold independence as a core measure of success. Furthermore, it aligns with Māori models of health that link the mind, the spirit, the connection with Whānau, and the physical world in a seamless and uncontrived way, which is important in the context of Aotearoa NZ.

As there is no one definition that adequately describes disability, due to its heterogeneity and intersectionality, PAMT draws on multiple disability definitions: the social model, the affirmation model, the minority model, the cultural model, the

\footnotetext{
${ }^{55}$ I considered calling it 'Crip MT'. However, I am aware that some people do not identify with the term "crip", for example, the keynote speaker, Paul Whittaker, at the 'Cripping the Muse' (2018) conference. Therefore, I sought a term that would include the work of all who address ableism.
} 
bio-psychosocial model, disability as identity, and the medical model. It recognises that different positions are available within each discourse that may be helpful at various times in reducing ableism. I have, perhaps controversially, included the medical model (albeit just). I recognise its shortcomings and its perpetuation of much ableism through its individualisation, deficit language, concern with norms, and pathologisation. However, I am certainly not against medication and medicine. I greatly appreciate the opportunities access to good medical intervention gives me. By keeping it in the mix of ways of understanding disability, one can draw on its focus on the particularity of the body/mind, which may be needed at times in creating understanding and reducing ableism. For example, if I explain to someone that my body doesn't allow me to walk far distances due to a particular diagnosis, they can understand why I'm declining their invitation to go on a weekend hiking trip. Furthermore, I do not wish to stigmatise conditions that require pharmacological intervention. For example, pain can become unbearable and require remediation. By using the term post-ableism, I do not intend to imply that all MT practice and theory prior to this was ableist. The term is used to name an orientation that identifies the need to undo ableist practices and for this to take a central focus in MT in a more overt way, as ableism remains under-theorised in MT. Having said that, ableist statements and sentiments were found widely in previous MT literature, practice standards and codes of ethics, which needs to be acknowledged in order to interrogate the violence of ableism and to speak of its injuries. Additionally, I do not offer PAMT as a way to undo what has gone before, but rather as a theoretical orientation that offers another lens to pre-existing theories. It is not an alternative but instead a way to extend our current models, to promote change within them and as Foucault would say, make them "groan" (Foucault, 1980, pp. 53-54). For example, one could apply PAMT within CoMT, use it as a lens within anti-oppressive MT or combine with aspects of narrative therapy.

I have used the term post-ableism as opposed to anti-ableism as the latter frames the practice in a more negative sounding way. As posthumanism is fundamentally optimistic in constructing new realities, it seems fitting for the term used to inspire practical (perhaps cautious) optimism and creation as well. Posthuman theory bases the ethical relation on positive grounds of joint projects and activities, not on the “negative or reactive grounds of shared vulnerability” (Braidotti, 2013, p. 190). 
However, like anti-ableism, I intend post-ableism to be a strategy, theory, action, and practice that challenges and counters ableism, inequalities, prejudices, and discrimination based on developmental, emotional, physical or psychiatric disability.

I have tussled with the use of therapy in PAMT. Like previous MT authors, I too wonder whether therapy is the right word for describing what I do. Feminism offers a critique of the label of therapy as being problematic due to the link with the patriarchal association of psychotherapeutic and medical models of therapy (O'Grady \& McFerran, 2006). PAMT would also be implicated in this way, as there is an inextricable and longstanding history of ableism within medical and psychotherapeutic practices. However, I have left the word therapy for now whilst acknowledging its problems. I may or may not find the right alternative. I note that the word therapy can have many different meanings. For example, in an article regarding Queer MT, Boggan et al., (2017) quoted a participant who said, "The therapist in my job title is not what therapist usually means. I'm not here to fix you. I don't think anything's wrong with you. Music therapist just means we're using music as a way to experience community and be expressive together" (p. 383).

Furthermore, because chapter 6 highlighted that the dichotomy of client and therapist can lead to unequal power relations, re-labeling clients might help to subvert the dichotomy. To this end, it may be helpful at times to borrow the term player from Rickson and McFerran (2014) to apply to everyone involved in the process. They describe player as an all-inclusive term that equalises and helps to reduce the hierarchy inherent in role titles.

\subsection{Ableism}

Before going further into what PAMT might look like, it is helpful to offer a definition of ableism and the forms it takes. The concept of ableism is not well understood (Wolbring, 2008). It is thought to have evolved in the 1960's and 1970's from the disabled people rights movements (Albrecht, 2006; Wolbring, 2012). One understanding often used is that ableism is a form of oppression that describes the negative treatment of disabled people and leads to disablism.

I find Campbell's (2008 and 2009) and Wolbring's (2008, 2012) definitions of ableism helpful. Both authors recognise that ableism can be another "-ism" but extend 
its scope and understanding beyond being related only to so called disabled people, making it applicable to many academic fields. Ableism is "a network of beliefs, processes and practices that produces a particular kind of self and body (the corporeal standard) that is projected as perfect, species-typical and therefore essential and fully human" (Campbell, 2009, p. 5). It is not just a matter of negative attitudes towards disabled people, but a deep way of thinking about bodies, wholeness and a striving for perfection (Campbell, 2014). Ableism expresses the ways in which the normative ideal of mental and physical wholeness is privileged in society: promoting an unobtainable corporeal aesthetic that encourages bodily projects towards that end. Disability signifies violated "wholeness" and incompleteness (Garland-Thomson, 1997) which has been ascribed as a negative cultural icon.

Like Campbell, Wolbring $(2008,2012,2009)$ contends that the dominant understanding of ableism is too limited and proposes a broadened definition. $\mathrm{He}$ defines ableism as:

... a set of beliefs, processes and practices that produce based on one's abilities a particular kind of understanding of oneself, one's body and one's relationship with others of one's species, other species and one's environment and includes one being judged by others. Ableism exhibits a favouritism for certain abilities that are projected as essential while at the same time labelling real or perceived deviations from - a lack of essential abilities - as a diminished state of being (Wolbring, 2009, p. 150).

He suggests that it is one of the most socially entrenched and accepted "-isms" and one of the biggest enablers for other "-isms" (Wolbring, 2008). Wolbring employs the concept of ableism as a tool to investigate other "-isms". Ableism is used to justify sexism through the favouring of certain abilities that women are labelled not to have, for example (Wolbring, 2008). He also uses it to investigate of the relationship between human and non-human entities, eco-ableism. Ability-based judgements are a factor that plays out in climate summits, for example. Expanding the concept of ableism to explore its interconnections with various human and non-human entities allows for the ethic of an increased relationality to be realised. Furthermore, he makes the point that ableism doesn't have to be negative, it just highlights that one favours certain abilities, but that it is often used to support negative actions and can lead to 
disableism, sexism, racism, and nationalism. Accordingly, I am not suggesting that it is a problem to celebrate ones' abilities and achievements in therapy, or strive for meaningful aspirations, but that it can become problematic when certain abilities are privileged.

\subsubsection{Benevolent ableism}

There are different types of problematic ableism that lead to disablism, "the discriminatory, oppressive, or abusive behaviour arising from the belief that people who do not have certain abilities are inferior to others" (Wolbring, 2009, p. 151). These are benevolent, internalised, systemic, and individual. Benevolent ableism is often subtle and is particularly important to consider within the context of therapy. It occurs when well-meaning people who don't intend to discriminate and intend to "help", are misguided by their own assumptions about what a disabled person needs and wants. They do not think of themselves as ableist oppressors, but through condescension, for example, they reinforce oppressive social relations (Cameron, 2009).

Well-intentioned therapists may attempt to normalise people with disabilities so that they will assimilate, rather than offering alternative positions. Benevolent ableism occurs when MT organisations promote services for disabled people without consulting those they deem to be helping. Or when feel-good stories are used in promotions that frame MT and/or the therapist as the hero in the story and the client's disabilities are centralised. As I have contested previously, it occurs when condescending labels are used, such as differently-abled or special needs. Labels that are thought to make disability more palatable can instead be condescending and belittling. The analysis illuminated issues of paternalism, which can lead to benevolent ableism.

\subsubsection{Internalised ableism}

Internalised ableism occurs when negative understandings of disability are internalised and applied to the self. Not identifying as disabled due to negative associations, or not wanting to spend time with other disabled people for the fear of 
being associated as one of "them" may express this ${ }^{56}$. Disabled people can often feel like a burden or undesirable as a result of internalised ableism.

I have seen the powerful effects of internalised ableism as a therapist when a player didn't want to publicly perform the music piece with her class because she felt she wasn't disabled like them and didn't want others to think of her in this way. She, of course, was not made to do something she felt uncomfortable doing. However, we recognised as a department, that we need to offer students examples of positive disabled identities and invited disabled speakers to talk with students the following year. Teaching about stigma was also implemented.

Drawing on analysis examples, internalised ableism may also affect disabled therapists in the way of disclosure issues. Therapists may not identify as disabled in attempt to separate their role from that of the client. Internalised ableism may make one view their disability negatively in relation to their practice, the maintenance of good practice being despite their disability instead of the possibility of good practice because of it.

\subsubsection{Systemic and institutional ableism}

Systemic and institutional ableism occurs within our social structures that do not allow disabled people participation. For example, it can occur within educational structures. Chapter 4 highlighted that accommodations can be hard won, and fairness related back to the normative body. Only some of the students I work with get adequate funding for accommodations and services.

Accessibility, including accessibility to music, is one of the main issues. For example, many music groups are not designed for disabled musicians. They may require music reading, take place in inaccessible buildings, not enough preparation time given to learn orchestral pieces, be paced too fast, go too long, and cost too much. Some blind students encountered inaccessibility to music notation as the musical letters $A$ to $G$ didn't correspond with standard Braille to the same letters, which was often a site of confusion for those learning both systems simultaneously (Challis, 2009).

\footnotetext{
${ }^{56}$ However, I also acknowledge that not identifying as disabled for reasons other than that of internalising negative associations does not mean that one has internalised ableism.
} 
Conventional instruments that rely on good fine and gross motor skills can be inaccessible (Challis, 2009). Often one needs to think outside of conventional instruments with technology-based instruments like midi interfaces, Makey Makey, or the soundbeam, which may be more accessible. Music therapists like Magee (2011, 2018) have written about this. MT can play a key role in creatively finding ways to make music more accessible. This is important because the significant underrepresentation of musicians with disabilities in the popular music industry might be partially counteracted by making music accessible at an educational level (Challis, 2009). Recently, I have been supporting students in accessing mainstream music classes at school within my role as a therapist, which is one part of changing the music environment and creating access.

Services and opportunities can be inaccessible due to cost or presumed ablebodiedness. Due to my disability I am unable to work full time. However, I am fortunate to have part-time wages and extended family that could pay for medical services when we couldn't. Many disabled people live in poverty and cannot afford therapy services. Therefore, disabling processes operating within society create barriers for disabled people to get as far as the MT room. The cost can be prohibitive. Accessibility issues of the profession extend beyond cost. For example, until recently, MT conferences in NZ presumed the able-bodiedness of therapists. The location was not considered in terms of access needs or attempts made to make the event accessible.

Two other forms of institutional ableism have particular relevance. The first is increased bureaucratic demands (chapter 5). There is endless paper work for disabled people and bureaucracy around specialist wait times and services one can access. The second form is that language-based therapies may be inaccessible if verbal language is not the person's mode of communication. MT is positioned to offer those who do not communicate verbally another way to engage in therapy.

\subsubsection{Individual ableism}

Individual ableism is often experienced through many microagressions that build up over time. The term microaggressions, Sue (2010) points out, was coined by African American psychiatrist Chester M. Pierce in 1970. At a later date, Sue (2010), explains microagressions as "brief, everyday exchanges that send denigrating messages to 
certain individuals because of their group membership" (p.xvi). Microaggressions were originally described in terms of racism but have been extended to encompass other marginalised groups experiences by Derald Wing Sue (Sue, 2010; Sue \& Sue, 2015). Hadley (2017) draws attention to microaggressions in MT practice in her important self-reflective chapter, which introduced me to reading the literature above.

There are three major categories of microaggressions: microassaults, microinsults, and microinvalidations (Hadley, 2017; Sue \& Sue, 2015). Microassaults are blatant intentional attacks of discrimination. Microinsults are comments or behaviours that unintentionally convey insensitivity or demean. A microinsult example from the analysis was the disregard of positive aspects of non-normative bodies/minds with statements like, "despite their disability". Finally, microinvalidations are behaviours or comments that dismiss, exclude, negate the disabled person's experience, thoughts, and feelings (Sue \& Sue, 2015). They include the erasure and trivialisation of disabled peoples' experiences. Previous chapter examples include being told, “you don’t look disabled" or to just "have a positive mindset" to overcome disability. This erases the ability to express a wide range of uncomfortable or confronting emotions, as there can be social backlash from marginalised voices that describe the realness of disability and disabling microaggressions.

In summary, microagressions are usually invisible to the perpetrator, unintentional and unconscious, allowing the power dynamic within interactions to be maintained (Hadley, 2017). Therefore, most people can identify blatant ableism, but microaggressions can be difficult to recognise. I experience direct or indirect microaggressions frequently throughout my day, which have a cumulative weight. For example, a colleague questioned the legitimacy of a supervisee's disability and another suggested that I eat a vegan diet to cure my illness. Therefore, barriers in PAMT refer to both physical and probably more so attitudinal barriers.

\subsubsection{Summary of the findings of ableism}

Having reviewed the ethical issues and power effects raised in the analysis chapters, they can be explained under the umbrella of ableism that led to restraining practices in my previous work or in the contexts I practiced/studied as a therapist. Some examples of practices that contributed to negative ableism highlighted in the analysis chapters were: 
- The expert paternalistic gaze: visual and aural observational truth making of disability privileged over the disabled person's embodied experience.

- Normative understandings of fairness in university education.

- The lack of flexible and adaptable university environments in some courses/departments. In such cases course designs, formats, and delivery were based on able-bodied/minded people.

- To date, 2017, the disabled body/mind is subordinate, potentially incompetent and othered in NZ professional regulatory processes (code of ethics and standards of practice).

- Dichotomies played a dominant role in the practice studied. These binaries contributed towards the enforcement of the able-disabled divide.

- The ableist tendencies of humanism had a strong presence in the MT practice studied. Independence, autonomy and control were privileged.

- The "human" was privileged over non-human entities, such as the environment, technologies, animals etc.

- Limited understandings of health and disability restricted understandings of success in an educational context.

- Ideas of success in supported learning in $\mathrm{NZ}$ were based on the normate individual.

- A "normal" way of being was privileged in the discourse of measurability in supported learning education: that of the active, doing, stable ablebodied/minded person. Some disabled ways of being were suppressed.

- The use of the EBP hierarchy of evidence disables people's subjective experiences of illness, disability, and therapy, as it is the lowest level of evidence.

It seems that all along my change in practice can be seen as heading towards the practice of PAMT. In fact, although it doesn't name it directly, the prelude to the analysis chapters at the end of chapter 3 was about the external and internal ableism that made it difficult for me to begin writing the chapters. I didn't have the understanding of ableism at the time to name what I was feeling.

Although previous chapters contain some examples of what I can now describe as PAMT, I will give further vignettes from my practice. These demonstrate how the 
creation of less disabling environments can be supported in MT processes through the four focus areas: The removal of barriers, supporting moments of connection, finding new/less restrictive spaces, therapist questioning their frame of reference. 


\section{PAMT vignettes}

\subsection{Vignettes demonstrating the removal of barriers}

\subsubsection{Devang}

Devang spoke fast, tripping over his words. The beginnings and endings were sometimes missed: it was his way of speaking. One had to learn how to hear him. But even then, it was not always possible to decipher. He was referred to MT to learn the difference between fast and slow, in order to learn how to speak slowly and clearly. Devang loved to sing. I noticed that he was already aware of what fast and slow meant and that he could sing in a slow or fast manner, but his articulation of words wasn't always clear regardless of pace. One day he asked if he could write his own song. While discussing what he would like his song to be about, I picked up on some of his ideas, but also missed much of what he was saying. I asked if he could speak slower, like the slow song we just sung. He attempted. He got frustrated. I suggested singing his ideas musically. He attempted. He got frustrated. I offered many ways to support him in altering his voice so that he could communicate exactly what he wanted. He attempted. He got frustrated. The songs we wrote were not entirely his words. I incorrectly at times had to fill in the parts I couldn't understand.

Understandably, he lost interest in songwriting after a few sessions. If I was working in neurorehabilitation some might say that he had lost motivation and became noncompliant in his voice work. Having witnessed the frustration he had experienced, I understood his need to say, "stuff it" and to take a break. Instead of trying to change his way of talking, I thought about how we could reduce the barriers that we faced in our communication. In talking with his speech and language therapist we introduced the use of touch chat (an iPad app) in his sessions. Through the use of technology, he was able to communicate what he wanted to say. During subsequent sessions he became an enthusiastic songwriter.

This is an example of supporting posthuman subjectivity by reducing barriers through our interdependence with non-human and human agents. Reducing the barriers of privileged normative speech to create a non-disabling environment through augmentative communication enabled Devang to be creative. 


\subsubsection{Lucy}

Lucy could play the piano well and wanted to play with others in her school community. However, she was unable to read music, which the chamber music group relied on as the main way of learning pieces. We therefore focused our sessions on aurally learning the music, so she could feel confident in playing the pieces and become a chamber music group member. At a later time, the group leader asked how she could support Lucy by adapting her teaching methods, resulting in Lucy requiring less support from MT sessions.

This is an example of removing barriers so that the student can access music making within her community. The community chamber group changed in response. For example, they got better at playing by ear and breaking the music into sections when learning, supporting the post-humanist value of interconnection. PAMT supports disabled people in accessing music making without it having to have a non-musical goal. The primary focus of Lucy's sessions was music making and ways to access this, not "the use of music to achieve non-musical goals" 57.

\subsection{Vignettes of supporting moments of connection}

\subsubsection{Lucas}

Lucas used to watch the chimes move while other people played them. Sally, another group player, had just started working with Lucas and seemed unsure about what to do. She stated that she couldn't sing and sat and watched the others. Her interaction with Lucas during the group was minimal. I suggested that she could hold the chimes so that Lucas could play them. The position in which she held them was not the right angle for Lucas' movements. We worked together to find the best place for her to hold them. Lucas swung his hand to make them crash while squealing "eeeeee". "That's it Lucas", Sally encouraged. The next session Sally offered Lucas the chimes. She also started squealing "eeeee" at the same time Lucas did. Lucas laughed when she did this, and it became a game that whole group participated in.

\footnotetext{
${ }^{57}$ A phrase often used in definitions of MT in NZ and one that I had used in explaining and presenting about MT.
} 
This is an example of how the less able aspects of oneself are viewed as a powerful source of connection in PAMT. Lucas required someone close to hold/position the chimes, and Lucas' different way of singing (“eeeee") provided Sally another way to use her voice during the session that was less songlike and less intimidating. It was, what could be viewed as, the incompleteness of Lucas' full range of movement and Sally's limited voice confidence that allowed this specific moment of connection to take place. The role of the therapist was to support how they could effectively work and create together.

\subsubsection{Patrice}

Patrice had shown a keen interest in my violin. I found a child-sized violin for her to use in sessions and also play throughout the week during break times from the classroom. We would improvise around the open string notes that she would bow. Patrice wanted to see the music we had made together, so I emailed some video clips to her parents for her to watch. Her father emailed back saying that the videos meant a lot to their family as Patrice's grandmother used to play the violin in orchestras. Her father contacted family to track down Patrice's Grandmother's old violin ${ }^{58}$. He shared how he had unearthed a lot about their family history from doing this. Patrice's interest in the violin connected her with her family and engaged them in the process of discovering new possibilities for Patrice that situated her within stories of her ancestors, connecting with two aspects of Te Whare Tapa Whā in particular-whānau and wairua.

\subsection{Vignettes of finding new/less restrictive spaces}

\subsubsection{Hazel}

Hazel's music was off the beat, sliding between tones and keys as if to never rest anywhere. Free from form, key and beat, we played whatever, whenever, however, but always with a mischievous grin and always together. It made me think of a quote by Siebers:

"Disability aesthetics refuses to recognize the representation of the

\footnotetext{
58 This aspect of the work had many parallels with my own story of the violin. My Nana played the violin and when I was interested in learning to play it she had her violin repaired for me.
} 
healthy body — and its definition of harmony, integrity, and beauty — as the sole determination of the aesthetic. Rather, disability aesthetics embraces beauty that seems by traditional standards to be broken, and yet it is not less beautiful, but more so, as a result" (Siebers, 2010, p. 3$)^{59}$.

This was a less restrictive space. I did not provide a beat for her to entrain to or settle in a key for her to come home to. I did not use the Iso principle. I came to the music without an agenda, then began to play, musician to musician (Kenny, 2015). Hazel did not have to conform to the western form of musical composition that deems "some notes consonant and others dissonant; some rhythms as falling on the beat and others off it" (Howe \& Jensen-Moulton, 2016, p. 526). She did not have to adhere to conformational practices that "dictate that one is either in tune or out of tune, blends with the ensemble or sticks out from it" (Howe \& Jensen-Moulton, 2016, p. 526). We celebrated every note, there was no hierarchy here: A supported disability aesthetics where Siebers (2010) describes how "the systemic oppression of disabled people would fail, and fail precisely because it could no longer be based on human appearances, features, and conditions deemed inferior" (Bakan, 2016, p. 548). Disability aesthetics considers cultural ideas and ideals of satisfaction and beauty through the lens of non-normativity (Howe \& Jensen-Moulton, 2016). By having an awareness of how music might enforce normalcy, I could understand how supporting Hazel's way of making music might reverse its dominance: perhaps even offering a disabled identity a place. The disabled aesthetic did not express defect but rather it enlarged "our vision of human variation and difference" (Siebers, 2010, p. 3). Such aesthetics and beauty can provide new, less restrictive spaces.

MT can therefore support one in escaping the dissonance between internal and external experiences and enable a space that might help to repair some of the injury of the chronic experience of misfit: the incongruity between mind and/or body and

${ }^{59}$ Siebers's notes that he did not write the book Disability Aesthetics to represent the exclusion of disability from aesthetic history, because no such exclusion has taken place. Rather his intent was to make the influence of disability obvious. Similarly, I argue that music therapists have not excluded disabled aesthetics but rather that its generative and transformative potential is not always acknowledged. Siebers argues that beauty always maintains an underlying sense of disability and that it is this presence of disability that allows the beauty of the creative work to endure. This links with the Japanese aesthetics of wabi-sabi, where beauty is imperfect, incomplete and impermanent. 
environment (Garland-Thomson, 2011). I hope that Hazel got a sense of that-from her motivation to create music we may have been on a helpful track. For me, I felt more "at home" in this music space.

\subsubsection{Penny}

Penny's teacher was concerned about what Penny would do after high school as she felt that Penny used her condition as an excuse to get involved in various things and focused on it too much in discussions. In MT she wanted to discuss the challenging aspects of pain (that was part of her condition), in between playing music-where she felt less pain for a short while. Penny felt silenced, "Nobody wants to understand, they don't want to hear how tough it is", she would say. Penny would request to sing, "Numb" by Linkin Park during these moments:

I'm tired of being what you want me to be

Feeling so faithless, lost under the surface

I don't know what you're expecting of me

Put under the pressure of walking in your shoes...

Every step that I take is another mistake to you...

I've become so numb, I can't feel you there

Become so tired, so much more aware

By becoming this, all I want to do

Is be more like me and be less like you.

Can't you see that you're smothering me?

Holding too tightly, afraid to lose control

'Cause everything that you thought I would be

Has fallen apart right in front of you...

After these moments where Penny could tell me how it was for her, she would request to play free music together. She explained that during improvisations she did not notice the sensation of pain, it would "stop for a bit". Penny's sessions perhaps provided a less restrictive space from that of having to censor what she said about her pain as well as temporary relief from the restrictive sensations of pain. PAMT can address the ableism imbedded in the censoring of disabled voices to fit within social 
conventions that makes some conversations feel impossible. Many MT practices support this well.

\subsection{Vignettes of therapist questioning their frame of reference}

The following vignettes place value on disruptions in my thinking. Such as when I perceived things as going wrong, when uncertainty was present, a new thought appeared, or when things were troubling/messy. This links with how the posthuman subject disrupts our vision of the human and that such disruptions are valued in posthumanism. Disability disrupts in the way that posthuman does, the presence of disability "disrupts, shakes up and interrogates the normative position...Disability inevitably disorientates normativity" (Goodley \& Runswick-Cole, 2014, p. 4). The following vignettes also demonstrate the need for a heightened reflexivity. As Baines (2013) explains, reflexivity is a political activity as it uses what we think, say, and do as the material for transformation (Allan, 2006).

\subsubsection{Callum}

Callum said he wanted to continue MT next year. He wrote in his report, "I'd like to play better than I already can. I would like to play the games better and it also brings friendship up from other people and it means others want to come and play with me, which is actually really rare" (appendix 2). Based on this, I continued to focus sessions around supporting relationships and social understanding.

One session Callum showed an interest in writing a song on GarageBand. He struggled to work collaboratively with the other students as he had specific ideas about how he wanted it to sound. After the session, I asked him how he found it. He said, "if I am working on my song, I do not want to bring anyone, because it makes it harder and they don't recognise the beat that I want. They are not mind readers". I initially thought, great he understands that they cannot possibly know exactly what he wants (which caused friction in prior sessions), but I felt that he still needed group sessions. I replied with, "Ok how about we alternate between one week with your friends and the other coming by yourself"? He responded, "No, how about the next month I come alone to work on my song". My initial reaction was to think that he had regressed and to continue to try and hold a space for the group work. 
On reflection, I reframed this as a progression in him understanding and voicing his needs and the need for me to support his way of being. I recognised that he doesn't have to like working with people all the time and that he had voiced wanting to be alone sometimes. His way of being required more alone time than my way of being. He came individually for the next five weeks and created a great piece of music he was happy with. The lyrics were:

There's hundreds of us! Some great others not, we all do one thing and that's free run.

Vaulting and climbing in the urban world!

I'm not the greatest and definitely not the safest,

But I gotta deal with this pain as it only makes me better

Treader and netter, come on let's move

Free runners, we are free men, not held down by artificial weights, we are the people of a new world, a better world!

Now come on let's make that better world!

He expressed, during this song, a better world "not held down by artificial weights". Was I initially one of those weights? He shared this song with his friends in a subsequent session and it provided a tool for him to generate conversation.

As well as being an example of a disruption and shift in my thinking, it also exemplifies how our limitations can offer other possibilities. Reframing his new limits around group work as a possible site of growth in another area - musical development. This vignette demonstrates my struggle against trying to restore a normal self within a player who is not necessarily biologically configured in ways that will allow him to ever meet the requirements of being socially 'normal' as these are currently constituted (Hodge, 2013). I shared my reflections and questions with him and in his notes, which parallels with the practice of narrative therapy.

\subsubsection{Marcus}

Marcus is a neurodiverse student who would get upset when having to leave the classroom on rainy days (discussed also in chapters $5 \& 6$ ). At the time of working with him, I was grappling with moving away from humanism towards posthumanism. 
If I thought about his sessions with a humanistic framework, I considered how they supported his language and communication. In particular how it facilitated opportunities to practice speech clarity and increase verbal interactions (speech is venerated in humanistic thinking). I considered how I could support independence by providing a motivating activity for him to leave the classroom for, without being piggy backed so his feet didn't touch the water. I saw how his autonomy, when it was raining, was facilitated through his control of the music. But ultimately, I felt that disability was seen as only lack when such humanistic qualities were worked towards.

Later viewing our sessions from a posthuman perspective, I considered how sessions could support enabling connections with human (me) and non-human entities: technology enabled an amplification of his voice through a microphone and an iPad provided an enhanced memory recall of the experience through video that we looked at after the music finished. His exploration of the weather and how to coexist with this non-human element enhanced a relational consciousness with his environment, which is important for survival and health. Thus, acknowledging the environmental humannature relationship. Marcus was singing about his relationship with rain, sometimes liking it, sometimes unsure about it. I considered moments of disruption as helpful, not as regression.

I felt that the posthuman reframing accepted his way of being. I could celebrate the interdependence of our music and more greatly appreciate that moments of anxiety around the rain were co-produced. For example, did we give Marcus enough time to fill his regulatory needs with self-calming behaviors on rainy days? Ultimately, I came to see disability as possibility.

However, to situate this in an NZ education context, I was required to work within the key competencies of "managing self", "participating and contributing", "relating to others", and "using language symbols and text" (Ministry of Education, 2019), which are currently based on humanistic thinking. There is a strong drive in the competencies towards independence and self-management. Therefore, I needed to reframe and rework the key competencies with a posthuman perspective. 
Table 2: Examples of potential key competency objectives for Marcus within humanism and posthumanism

\begin{tabular}{|c|c|c|}
\hline & Humanism & Posthumanism \\
\hline Managing self & $\begin{array}{l}\text { Marcus would work towards } \\
\text { independently going outside } \\
\text { in the rain. }\end{array}$ & $\begin{array}{l}\text { Interconnected relationships } \\
\text { would be explored that may } \\
\text { assist on rainy days. }\end{array}$ \\
\hline $\begin{array}{l}\text { Participating } \\
\text { and contributing }\end{array}$ & $\begin{array}{l}\text { Would strive towards active } \\
\text { participation as a sign of } \\
\text { success. }\end{array}$ & $\begin{array}{l}\text { Would celebrate both active and } \\
\text { passive participation equally. }\end{array}$ \\
\hline $\begin{array}{l}\text { Relating to } \\
\text { others }\end{array}$ & $\begin{array}{l}\text { Marcus would work towards } \\
\text { furthering his social skills } \\
\text { development and peer } \\
\text { relationships: Work towards } \\
\text { having peers join him in } \\
\text { sessions. }\end{array}$ & $\begin{array}{l}\text { To facilitate everyone's social } \\
\text { understanding. To explore } \\
\text { relationships with multiple } \\
\text { others (human and non-human) }\end{array}$ \\
\hline $\begin{array}{l}\text { Using language } \\
\text { symbols and text }\end{array}$ & $\begin{array}{l}\text { Work towards speech clarity } \\
\text { and normalised productions } \\
\text { of speech (speech is } \\
\text { venerated). }\end{array}$ & $\begin{array}{l}\text { Would support an embodied } \\
\text { and impure activity based on } \\
\text { noise, relationality, and } \\
\text { reciprocity" (St. Pierre, 2015) }\end{array}$ \\
\hline Thinking & $\begin{array}{l}\text { To share thoughts, ask } \\
\text { questions, independently } \\
\text { complete tasks. }\end{array}$ & $\begin{array}{l}\text { To support creativity and } \\
\text { embrace disabled aesthetics. }\end{array}$ \\
\hline
\end{tabular}

\subsubsection{Olive}

I do not like to admit this, but I had become bored working with Olive. I initially thought her repetitive music choices were the reason. I reflected later that my boredom arose partly through frustration at what seemed to be a repetitive therapy process with little change. Like most western therapy, mine is embedded within a 
culture of transformation narratives, such as the formative account of Dibs and play therapy (Axline, 1964). In such narratives children with autism, through therapeutic intervention, make miraculous breakthroughs into social and emotional normality (Fisher, 2007; Hodge, 2013). I was not experiencing such dramatic changes with Olive. Perhaps this made the sessions somewhat disappointing and less engaging for me personally? (Hodge, 2013). The problem of my boredom didn't stem from Olive's repetitive sameness but from larger social discourses and expectations.

\subsection{Further thoughts on vignettes}

I note that there are many more examples of using a PAMT orientation. PAMT is context specific; therefore, it will change across contexts and therapists and might look quite different to the examples given here. I also acknowledge that even within the examples given there is room for growth and development in working in a postableist way. Across all focus areas that may support the creation of a less disabling environment/experience, an extended relational ethic was important. 


\section{Chapter 9}

\section{Creating the Self as a Work of Art (Part Two): Changing}

Subjectivity Through Illness/Disability, Music and Research.

"Becoming an other": Photograph taken at the National Gallery of Victoria, 2013

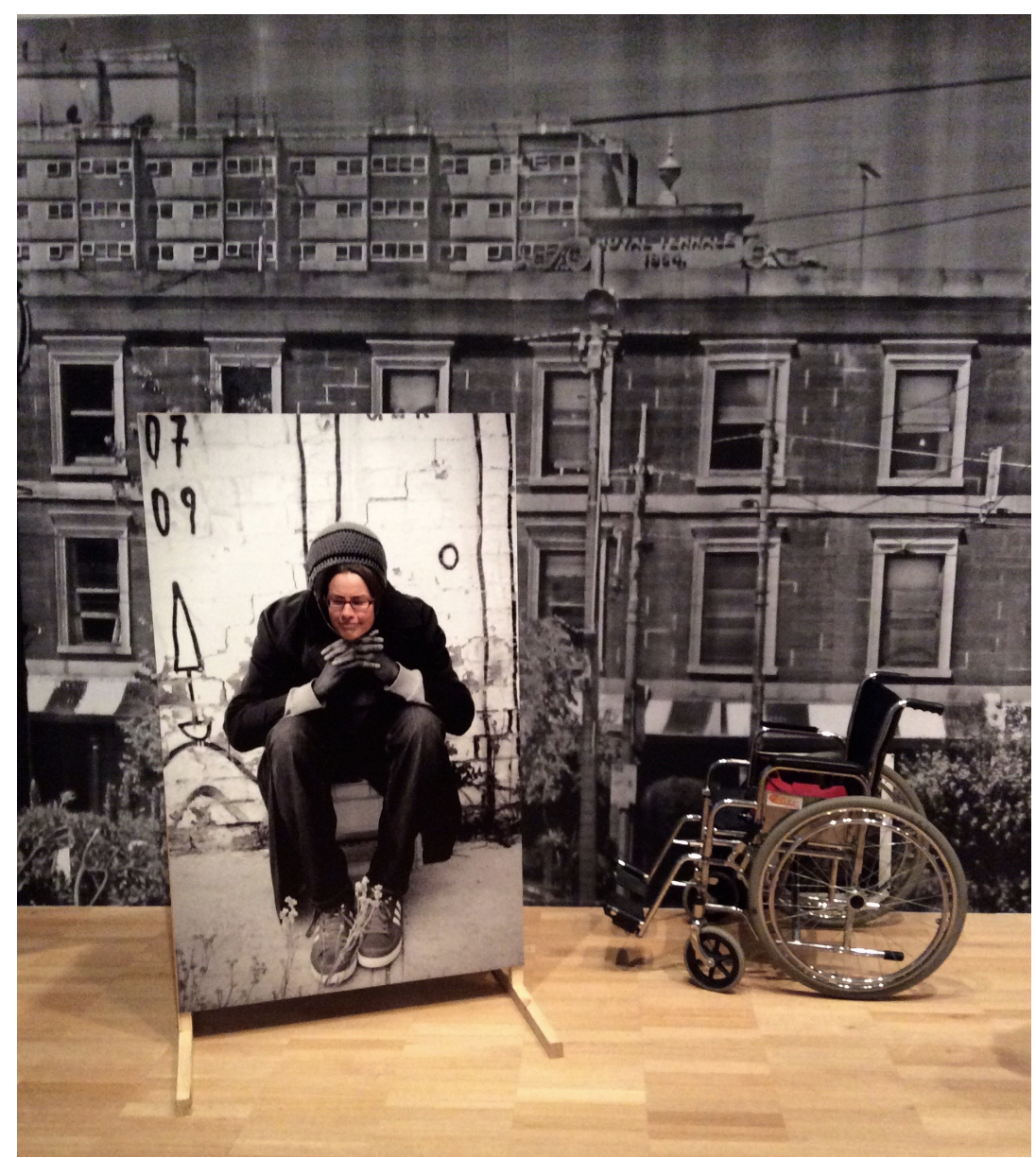

[Photograph of my face in a picture of a person sitting down. Background of an apartment block. Wheelchair in foreground] 


\section{Introduction}

"But the question may be asked: Are not the kinds of phenomena that I have been discussing of interest precisely because they produce us as human beings with a certain kind of subjectivity" (Rose, 2000, p. 320).

The previous chapter primarily explored creating my professional identity and practice, which resulted in the development of PAMT with an expanded relational ethic generated from aspects of posthumanism. This chapter furthers the process of creating the self as a work of art by extending the idea of agonism as an important aspect of the relational ethic and looking at how my personal subjectivity/identity ${ }^{60}$ has changed during the development of PAMT. That is, I was compelled to look at how my own subjectivity has developed in response to confronting ableism in my practice.

This seemed relevant for three reasons. Firstly, how one practices can have impact on personal experiences and subjectivity/identity and vice versa. Secondly, how we understand disability may impact on the client's subjectivity/identity. Thirdly, it demonstrates the discursive and cultural construction of disability (Straus, 2011), reminding that we as therapists can play a role in its assemblage. Because my experiences of illness/disability and MT practice are co-constructed, the findings from this chapter have relevance to many of the research questions, particularly around how the work of therapy can be managed and the potential of poststructural autoethnography.

Feminism's standpoint theory is a useful concept to frame this chapter. It reinforces the multiplicity of all disabled women's identities, histories, bodies and minds asserting that individual experiences constitute subjectivity from which they perceive and speak (Garland-Thomson, 1997). Just as standpoint theory has reformulated gender identity, disabled identity too can be understood within this theory as a complex, dynamic web of interrelated, often contradictory experiences, approaches,

${ }^{60}$ Identity is understood in this thesis not as a fixed entity but as an emotionally charged discursive description of ourselves which is subject to change. Subjectivity is the processes by which we become a person and the condition of being a person; how we are constituted as subjects (biologically and culturally) and how we experience ourselves. 
and attributions mediated by culture and individual history (Garland-Thomson, 1997). For example, a change in my body and physical possibility changed subjectivity itself because my standpoint had changed. My previous non-disabled standpoint in the early years of my practice had been shaped by the normalisation of humanism, normalised music, and medicalised identifications of disability. The dominance of these discourses concealed alternative positions, causing a disjuncture with the kind of subjectivity my changed bodily experience called for. My subjectivity transformed during the research process and in response to life events.

From my change in standpoint, the identity and subjectivity created in this chapter is that of the discontinuous other. At the time of writing, my experience had been transformed by discourses of posthumanism, disability culture and Crip music. I never found myself within each discourse but instead, from the discontinuous and incomplete self, I found many selves in many places that helped in different ways to subvert aspects of ableism. Often, I remained in the tensions of different discourses. Due to this, agonism is another helpful Foucauldian and feminist concept, as it understands the struggle between ethical standpoints as a potential site of productive possibility (agonistic struggle). Meanings and practices are continually negotiated in the public, political arena, where they are subject to open debate and conflict, as opposed to holding essential principles (Cloyes, 2002; Mouffe, 2016). Agonism recognises ethical pluralism, the understanding that ethical systems are diverse and heterogeneous. This chapter demonstrates that my ethical care of the self was constructed through contestable and negotiated discursive disabled politics. Agonism allows for the possibility that things could be otherwise. In short, this chapter posits the need to let go of quests to find ourselves and others and instead appreciate many selves for the self existed nowhere in totality. This has implications for PAMT: the need to foster multiplicity and many positions within practice to support an agonistic ethic. This ethic links with PAMT's principle of therapists questioning their frame of reference.

This chapter explores the changes in my own identity and subjectivity through the visual analogy of floor plans and music compositions that I composed at various times. It demonstrates how I both destabilise my identity and claim the particularity of my body and experience to manage negative aspects of disability. 


\section{Constructing a new home: Changing subjectivity through illness/disability, music and research to subvert internalised ableism.}

Prior to illness, my subjectivity regarding illness/disability was constructed primarily through humanistic discourse, discourses of normativity in music, and the medicalised understandings of disability. These dominant discourses had a strong presence in the MT profession in Aotearoa New Zealand (NZ) and/or in day-to-day life. Other discourses had a smaller presence. If my subjectivity were a house it would look like the diagram of the floor plan below. The size each room is relative to the approximate amount of time I spent occupying positions within these discourses.

\section{Diagram 1: Floor plan of initial subjectivity}

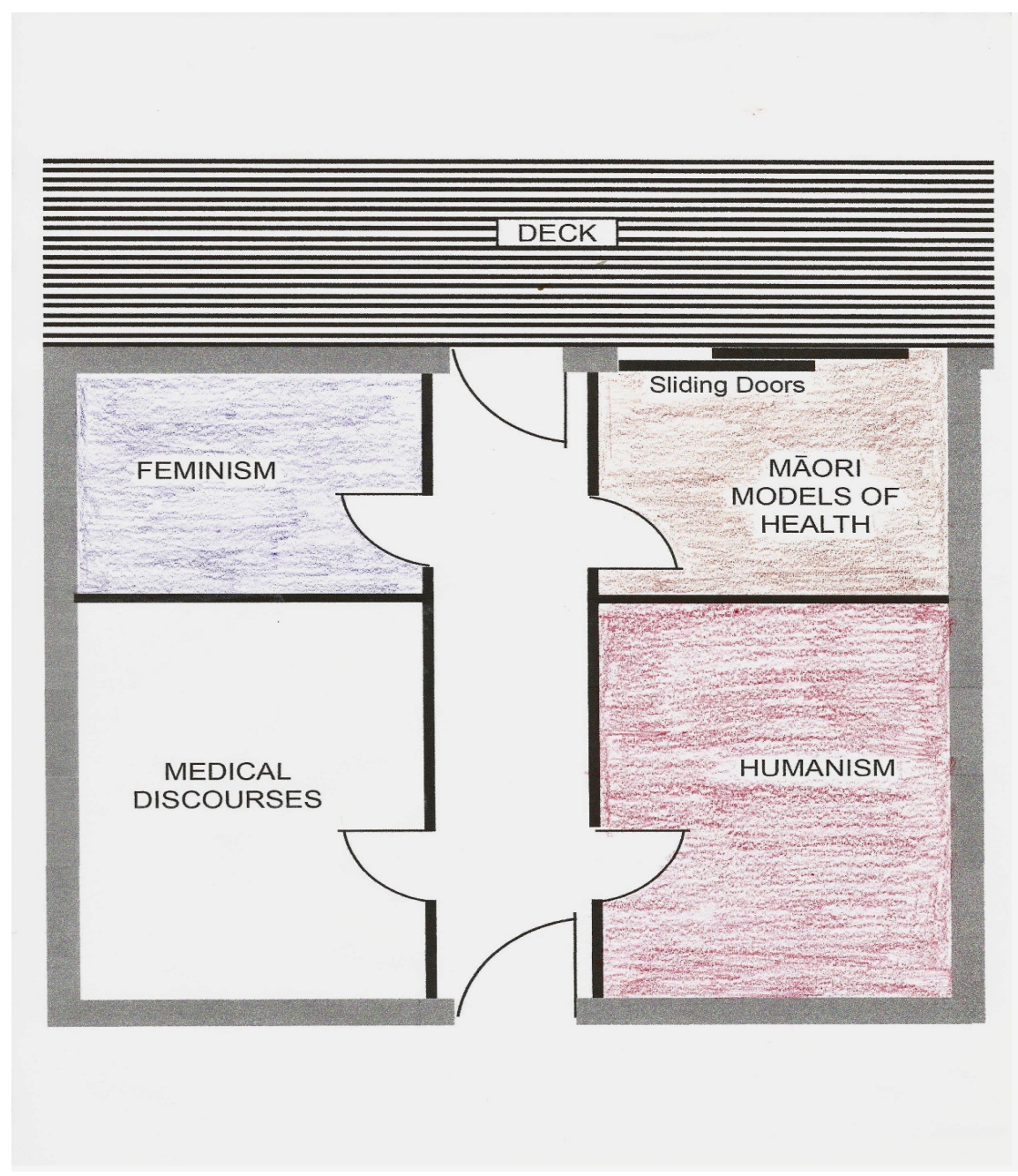

[Floor plan with two large rooms (humanism, medical discourses) and two smaller rooms (feminism, Māori models of health)]. 
My floor plan has undergone many changes during the years of illness in response to ableism, discrimination, and to escape the negative aspects of these discourses on my identity. My old floor plan felt an "unhomely" space. Manathunga (2007) uses the postcolonial metaphor of unhomeliness, derived from Bhabha (1994), in describing academic development and academic developer identities. Here, it captures my desire to change and recreate the space to make it homelier. I have added rooms, increased the size of smaller rooms and decreased larger ones. Currently the diagram below represents where the renovations have got:

\section{Diagram 2: Renovations of subjectivity floor plan}

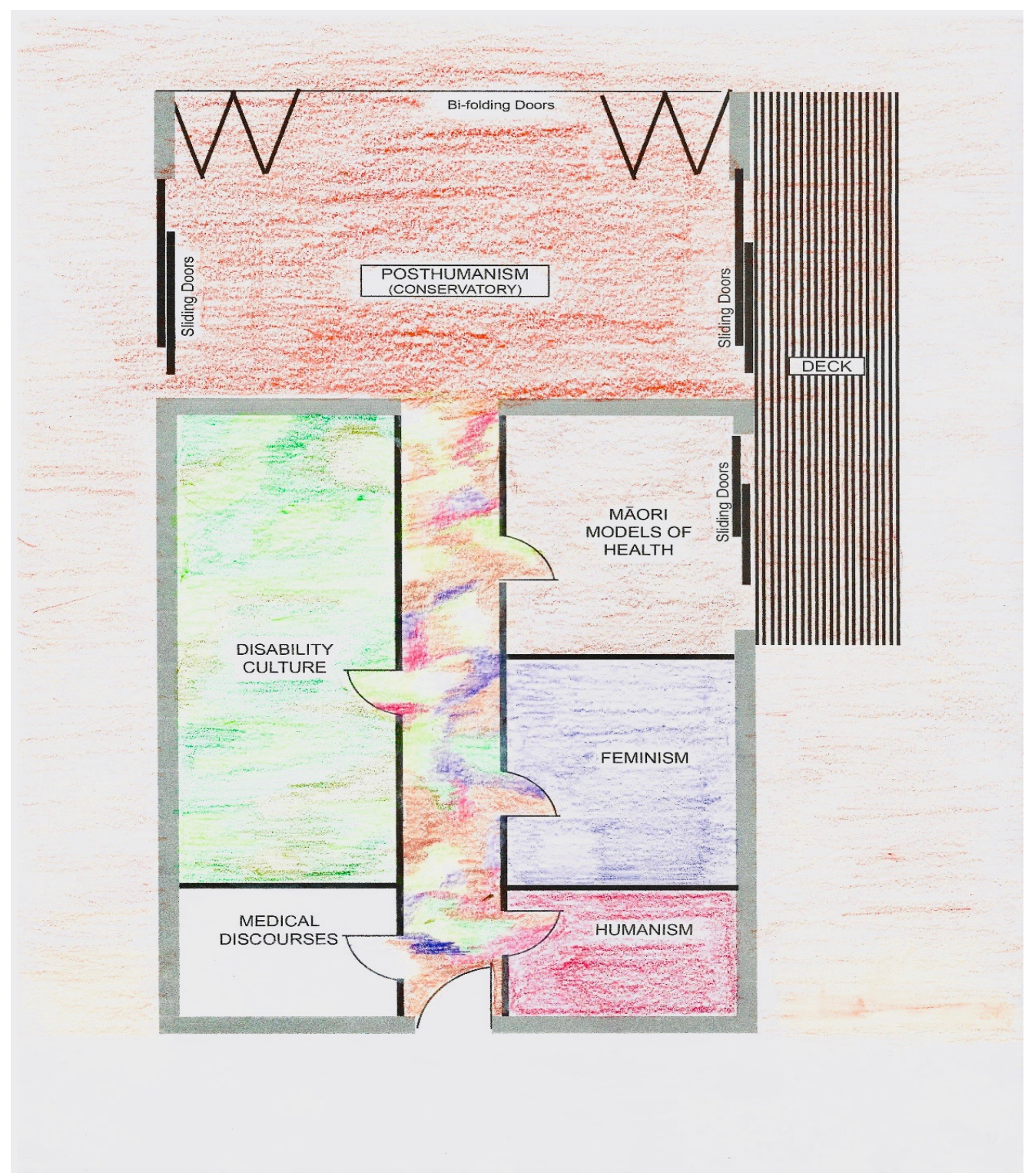

[Floor plan with two large rooms (disability culture, posthumanism), two medium rooms (feminism, Māori models of health), and two small rooms (medical discourse, humanism) and a colourful hallway]. 
The diagram represents the current discourses that primarily impact on my subjective understanding about illness/disability (larger rooms) and some of those which have less influence (smaller rooms). I recognise that I haven't included everything. Furthermore, the floor plan metaphor draws on the poststructural understanding that the self is not given to us but instead constructed. Therefore, poststructuralism is the wiring of the house.

The focus of the descriptions below is mostly concerned with negotiating disabled identities. Other aspects that impact on identity, such as pākehā, heterosexual, mother, low-income family, cisgender, and spiritual but not religious, are not explicitly discussed, but nevertheless interact within the various discourses described. I may have leaned towards different discourses when reconstructing my home if some of these aspects of identity were different. This reconstruction has taken place through experiencing illness/disability, music-making, and undertaking research, which opened up different positions and discourses to inhabit.

I have chosen to only discuss the main discursive pairs that created tension and therefore provided sites agonism in myself and work. These are:

- Medical discourse and disability studies

- Humanism and posthumansim

The tensions between humanism and posthumanism, and disability studies and medical discourse, became important in agonising a post-ableist practice and care of the self that was less restrictive and full of possibilities. This did not call for a reconciling of the discourses because within agonism the tensions themselves are productive and possible starting points for developing an effective political postableist theory of practice (Cloyes, 2002).

Furthermore, I have not described all of the rooms because the aim was mainly to demonstrate that different positions are taken at different times, some positions are taken more often than others, multiple discourses may construct one's subjectivity, there are tensions within and between discursive selves, and the discourses that we find helpful change over time and are influenced by bodily experiences. Indeed, I intend to show some of the complexity of identity negotiation. It is not possible to capture my subjectivity/identity-it is intricate, complex, and always on the move. 
This is just a "floor plan", not an actual house so to speak. The narrative descriptions capture where I identify within certain discourses and where I do not: the many different identities I occupy at different times and how they can feel. The rooms/discourses discussed are accompanied by examples of music compositions that I had previously written at various times. The examples match particular discursive ways of being.

\subsection{Room one: medical discourse}

Entering the front door, I find myself in the room that many people first visit. The room is white, pristine and clean: My reflection looked back from the souvenirs from a past life. The life I was reminded to get back to. A striving of a valued representation of myself in relation with the nondisabled majority (Garland-Thomson, 1997). I sat here awhile: "Many disabled people at one time considered themselves nondisabled" (Garland-Thomson, 1997, p.15). I was just sick. Temporarily. Momentarily. Moments turned into more moments.

At first, there was something comforting about the order and neatness of this room. Everyone seemed to know what to do within it. They would direct me. I needed them to for I did not know what this was and what to do: to relieve the discomfort from a body screaming. I followed. What else was I to do: they spoke in a language I had never heard before: pronounce "ganglionic acetylcholine receptor autoantibody".

The longer I stayed in this room, the more I started to devalue myself: a spoiled identity (Goffman, 1963). My body created mess. Some of the mess they couldn't tidy up into neat categories and the rest they split off into many. I struggled to identify with the names given. Names here are always changing. Had I been in this room 10 years ago, the names given may have been different. Some illnesses once felt entirely real in medicine exist no more (e.g., hysteria, neurasthenia, fugue, or nostalgia-all once legitimate medical diagnoses), and others that never existed before as precise categories now seem all too real at work (e.g., attention deficit disorder or autism) (Straus, 2011). What will they call my things at a later time? Within the unbalanced power dynamics stigma can be felt here. I didn't know what they knew. What they knew was most important. But the messiness my body created called bluff on their certainty. 
I can't stay long in this room of being "the patient", for "if the self we see reflected in the eyes of others is devalued, there is little incentive for social interaction" (Green, Davis, Karshmer, \& Marsh, 2005, p. 2010). I stopped wanting to talk to them. I took a four-week break (or more) from their scheduled weekly viewing appointments.

I still visit this room sometimes, albeit not if I don't have to, to relieve some of the bodily aches that other rooms can't adequately attend to. To deny that this room is here, would be to deny a body struggling and stigmatise the "unhealthy disabled" (Wendell, 2001, p. 17). Sometimes I can stand in the hallway to access what is in this room. Some doctors step out at times and resist the ableist notion that some bodies know, and some bodies don't, some bodies are valued, and some are not.

\subsubsection{The music of room one}

Music has a restrained feel in this room. Prior to illness, I wrote music here for a long time: complete works, structured, and coherent. Informed by Western normativity of harmonic understandings and rhythmic techniques (Audio 2 example:

https://soundcloud.com/user-419330485/audio-1-example). I struggle to write in this way now. My body interrupts the finishing, calling me to take breaks or to abruptly stop writing. The music playing in this room was another distressing portrayal of my defectiveness, for I was not ordered or complete like these compositions. I lacked wholeness and fluidity in relation to it.

Standards. The standards started to exclude. There is a best way for singing to sound and music to dance. In my MT practice I rehearsed with the students multiple times to make sure their music was up to scratch. There was the parent who asked if I could support her child to sing in tune. My identity here became more and more out of tune. There were standards I could see many struggling to meet. I escape making music in this way when the failure feels too much.

Sometimes, however, I still make music this way. There are after all some benefits. People see competence and ability when normative music standards are met, subverting held notions of what disabled people are capable of and the idea that disabled people are only that. I also make music in this way to connect and relate with friends, family, and anyone else who prefer this. A shared activity when I can. 


\subsection{Room two: Discourses of disability culture and positive disabled identities}

The room is green (green has the most shades of any colour): there are many styles and patterns. I can't find my past reflection anywhere. Life is different. Creativity is a large part of disability culture ${ }^{61}$. My illness induced adaptation and has been the source of creative responses (Carel, 2013). Here most people say that they are disabled. I had to learn how to say this. Roughly 15 precent of the world's population has some kind of disability but only a fraction of those people actually identify themselves as people with disabilities (Bogart, 2017). Rejected due to its stigma. Learning to say disabled was hard outside of this room: A friend said, "I was shocked the first time you referred to yourself as disabled". At work I am enticed not to use the D-word when referring to people who are disabled: disabled students are still referred to as people with "special needs" the majority of time. Implying the word disability is just too awful to say, sending the subtle hint that one should be ashamed of it. But, in this room I am bolstered to claim it for myself.

When reading Cameron (2009), I came across Crescendo's blues number, 'Disabled People aren't Allowed to Say "Fuck" (Holdsworth 1989a, p. 9): A written piece in response to his performance at a residential home where he had been told to mind his language. The blues makes the point of naming terms through which disabled people are encouraged by mainstream culture to recognise themselves (Cameron, 2009). I wrote a journal entry in the style of Crescendo's blues number in response:

Outside this room,

I am allowed to say, chronically, severely, special, assistance. But I am not to say I'm Disabled.

I am allowed to say, I'm trying, I'll fight this, I'll beat this, I'm not this. But I am not to say I am disabled.

${ }^{61}$ Disability is a broad, heterogeneous and diverse identity. However, disabled identities are united in understanding disability as being shaped by culture, which includes music, and the shared experience of discrimination. 
I am allowed to say, treatment, hope, charity, spirituality. But I am not to say I am disabled

I am allowed to say, difficult, challenging, pain, fatigue. But I am not to say I am disabled.

I am allowed to say, help me, support, accommodations, visitations. But I am not to say I'm disabled.

I am allowed to say thanks, offer inspiration and answer invasive questions. But I am not to say, "I'm disabled".

I am allowed to strive for independence, autonomy, bodily control. But I am not to be disabled.

All while you disable me. We disable me. They disable me. It disables me. I wouldn't be so disabled, you know, if I wasn't unable to say, "I'm disabled".

But here in this room I say,

I am disabled. I say ableism, oppression, inequality, injustice I am disabled. I don't overcome, live despite, exceed my limitations I am disabled. I find creative solutions, interdependent relationships, bodily acceptance.

I am disabled. I shrug off degrading views of disability, as it is theirs not mine.

I am disabled. I am disabled. I am disabled.

There was strength gained in this room to speak up. Someone was belittling a person with a disability; I claimed disability in order to make the point that the treatment was not right. The answer to which I got was “but I wouldn't say that about you, as I do not see you as disabled": A microinsult that demeans a person in the form of a positive compliment based on biased/stereotyped attitudes (Sue, 2010; Sue \& Sue, 
2015). I was praised while those societal, "not me" figures were insulted. I am still "one of you" as long as my impairment is considered to be minor or temporary (Green et al., 2005).

In this room, my group identification grew from disabled activists within my community of patients with similar diagnoses. The readings from disability studies that reinforce the walls also supported me to take the position of rejecting a stigmatising culture and identifying as disabled. Like Simi Linton's (1998) concept of "claiming disability", I reclaimed language, such as crip, disabled and spastic. "Have you been in the wars" the stranger remarked as I struggled down the street with what the neurologist called a "spastic gait". "No, I'm just spastic" I replied. I did it to shock. Self-identifying can help build resilience and can be critical for self-acceptance and self-esteem (Bogart, Lund, \& Rottenstein, 2017; Bogart, 2017). In this room I could somewhat reduce the stigma through reframing labels, reducing the assaults on my self-esteem. A disabled identity is associated with lower depression and anxiety (Bogart, 2017).

I felt the social support from those who shared disability status, yet there was one thing I struggled with: disability pride. Disability pride postulates that a disabled identity should be "positive and affirming, a source of distinct value, unique perspectives and group solidarity" (Bogart et al., 2017, p. 2; Gill, 1997; Swain \& French, 2000). I am still working through and mulling over how proud I am. Am I proud? There are many meanings of pride: Disability pride is no different.

Pride, $n$. "a feeling of deep pleasure or satisfaction derived from one's own achievement"'(Oxford Dictionaries, 2018).

I am not proud in this way: I did not knowingly catch the virus that stressed my immune system into hyper drive. I did not build the wall that it crashed into at full speed, fracturing some parts off in the wrong direction. I did not will this, ask for it, or pray for it to become part of me. It was never an achievement.

Pride, $n$. "Consciousness of one's own dignity" (Oxford Dictionaries, 2018).

I am proud in this way: in not accepting incompetent health care and educating others when needed; in repeatedly asking for the resources needed to participate; in 
not allowing the tears from one comment to put me off asking for help again. It is an exercise in asserting worthiness.

Pride, $n$. "The quality of having an excessively high opinion of oneself or one's importance" (Oxford Dictionaries, 2018).

I am not proud in this way. I am not more for the diagnoses added to my name-they are weightless. I am not less for the ableism that surrounds me-it does not subtract.

Pride, $n$. "A group of lions forming a social unit" (Oxford Dictionaries, 2018).

I am proud in this social group way. To move to call for a legal foundation for accessibility in NZ. To move to create access to medicines not funded by Pharmac. A social movement.

But to speak of pride is to bring up shame. When I know of the pride in managing difficult physical symptoms, then I know of the shame when I snap at someone due to pain shortening my wick. When I know of the pride in standing up for the inequalities of disabled people, then I know of the shame when I was too fatigued to join the rally and remained quiet. When I know of the pride in voicing a nonnormative perspective, I know of the shame in not seeing the normalising waters I swam in. I am not just proud.

I wish to subvert this binary of pride and shame. In this way, I'm not sure I fully belong in this room if I can't bring myself to say "I'm proud" all of the time.

Sometimes my body doesn't belong. As the level of my bodily impairment decreased in 2017 so too did my felt "authority" to speak so loudly. More ally than in-group cultural member. I was quietly disabled; the assaults of ableism and embodiment had lessened. Research demonstrates that the level of impairment and the experience of stigma are the biggest factors in whether someone with a disability identified that way (Bogart et al., 2017). Sometimes there doesn't feel enough space for the realities of fatigue here or within pride to speak of the pain. A silencing form of ableism. A positive identity, but perhaps too positive at times when the body crumbles? 
I have spent long periods of time in room two and come often. I have learned much and undone many unhelpful ableist norms. It is perhaps one of my more preferred rooms before I return to the hallway.

\subsubsection{The music of room two}

The air is filled with non-normative sounds. Late Style, an aesthetic category that is described as having qualities such as fragmentation, intimacy, nostalgia or concision (Straus, 2011), plays here. Straus (2011) contends that there is a correlation between

Late Style and the composer's experience of non-normative bodily or mental function. Their disabilities are inscribed in their music, which correlate with Late Style. Due to this, Straus understands Late Style as Disability Style as the works he studied narrated stories of disability. This happens to sometimes coincide with the older age of many composers, as this is when they experienced fractured minds/bodies. Some of my compositions written during illness were compressed miniaturised pieces, fragmented, discontinuous, interrupted, and non-harmonious. There were some that were difficult: bitter, tense, and unconcerned with pleasing (audio 3 example: https://soundcloud.com/user-419330485/audio-3-example-dontgive-an-f). Some were austere: bare, expressionless, immobilised. Perhaps in some ways I became more creative? These qualities match the descriptions of Straus's Late Style/Disability Style. Some characteristics are contradictory, possibly due to the internal contradictions encountered by disabled composers (Straus, 2011). I observe the lateness of my arrival here: in fully appreciating and celebrating disabled aesthetics.

I make music this way when normative ways become inaccessible. At times, I couldn't be integrated back in to my old music groups I attended prior to illness. Their rehearsals were too long and demanding physically. Crumpling in the corner, only sometimes participating, was not an easy option. But in this room, I crip music making. Short snippets. Bursts. Explosions. Silences. Many bars of rests. Slower. More interruptions. No goal of perfection. Imperfections valued. Valued. A valued identity-or more excitingly a rebellious one! There isn't much down side to making music here and I do so often. Except too much cripping can cause a disconnect with others: Folk who I love and value. 


\subsection{Room 3: Humanistic discourses ${ }^{62}$}

This room is red. Alarming. Disarming. It did not used to be this way. Previously, it was big, with red velvet curtains fit for royal status. It had a throne to sit on as it often ruled. It has now shrunk. Most days, I want a revolution and the throne over thrown.

Certain bodies and minds are privileged here. Others are not. I have never felt less than human until I became one of the non-independent; one who needed my father as a caregiver to cook and clean while my husband was away and one who relied on benefits when my ability to earn decreased, for example. Within this room the disabled figure represents the incomplete, unbounded, and compromised body susceptible to external forces (Garland-Thomson, 1997). My body mocks the ideals of self-determination and autonomy. No matter how much of it I have, there is not enough self-determination that can overwrite antibodies or keep me upright longer than the heart and brain can manage. I will not take too much breath in this place where ableism breeds.

I visit for one reason only, when needing to claim human rights within the structures already in place. Many established human rights processes rely on humanistic discourse. Sometimes it's the only way I can see to improve things: A political position to move: an identity of the humanistic human for the right to be considered.

\subsubsection{The music of room three}

The music in this room is human made: humans play the instruments and the composer wrote every note. When performing/recording the music, a silent environment was created so that no other sounds could interfere. The human is front and center and in control (audio 4 example: https://soundcloud.com/user419330485/audio-example-5).

\subsection{Room 4 - the conservatory: Posthumanism}

The large conservatory is orange. Once removed from red, yet quite different. It is large, growing, and always changing. There is no door to the hallway. All the colours of the hallway can be easily accessed here. Big glass sliding doors open wide. Inside

${ }^{62}$ The description of this room is comparatively shorter than the other rooms. This is because my tussle with humanism has already had much focus in chapters 6 and 7 . 
and outside become one. Wall boundaries have gone to allow for greater interconnection-human and non-human live together. Here, we are more. Not contained, restrained, but extended. We are the environments we inhabit, the technology that aids us, the connections with others, the non-humans that co-exist. All disabled. All reliant. Here, I do not feel bad for holding my husband's arms to walk, or my technology derived medicines that plant miracles in my blood, or the receiving of benefits made from other's taxes, or for simply taking in the air that lives temporarily in my lungs. There is a celebration of interdependence here, not a shaming of it, or a striving away from it. All bodies are accepted with its central demand to not be pressured by society to adopt a normal body (Goodley, Lawthom, \& Runswick-Cole, 2014; Wolbring, 2009; 2012). A humble and connected identity, perhaps? The conservatory has much to offer. Like the greater awareness it brings to environmental problems that threaten our survival through exposing ableist understandings of the non-human that contributes to inaction. Humanistic abilities aren't centered as the most important here, espousing a non-hierarchical valuing of all human and non-human entities.

However, I leave the conservatory when the relationship with technology is not properly considered in relation to disabled people. Considerations need to be made regarding the likely equity, equality, and social consequences of any new technology (Wolbring, 2009). Posthumanism has been critiqued as not articulating a comprehensive ethics for how individuals should respond to technology (Benko, 2005). I feel uncomfortable about technologies' ability to identify and terminate a life that could have been well lived, given the chance. Although a female's choice over her body is important, such as during pregnancy, I wonder what choices we are making in a society that devalues some lives? Technology has led to a new group of marginalised people (Wolbring, 2009). For example, human enhancement technology may be enabling for a few but cost prohibitive for many. The posthuman intends to value all kinds of lives, which needs to remain central in considerations of the use of technology and its potential inequalities. 


\subsubsection{The music of room four}

Interestingly, my compositions had become more posthuman since becoming unwell. The use of music technology to facilitate and produce compositions had increased. It was less fatiguing for me to utilise the electronic instruments in the music software programme than to record myself playing live instruments. Technology facilitated my music engagement. My husband brought me sound recordings from places he had been, such as China, when I wasn't well enough to travel with him. These recordings became part of my compositions: connecting with environments and life experiences I couldn't otherwise connect with. The compositions, therefore, are not just my own, they were interdependently created with people and the environment (audio 5 example: https://www.youtube.com/watch?time_continue=2\&v=FDXbkeIeo30) ${ }^{63}$.

\subsection{Hallway}

Although I spend a lot of time in some rooms and minimal in others, it is perhaps the hallway that I occupy the most: the between space. Here, where the holes from the doorway frames congregate, aspects of each room escape, meet and connect: The space that forms my current subjectivity. I am a partial carnivalesque figure (without the disfigurement). I have the right to be other and defy categories that don't quite suit. I see the underside of the subjective positions in the discursive rooms (GarlandThomson, 1997). I am one of a novelty, falling in the between spaces of medical diagnoses and theoretical categories. I am ability fluid ${ }^{64}$ : a dynamic mix of disabled and abled identities that destabilise the category boundaries through the changeability, fluidity, and the inseparableness of each state. I fall between discourses that lock me in to one camp or the other. I am a discontinuous other, "producing knowledge within and against academic intelligibilities" (Lather, 2006, p. 41). In the hallway I can both critique binary producing identity politics as well as being dependent on them at times. Here I find myself in the tensions that characterise fields of knowledge. Due to this, there were often no words to describe myself in these between spaces. In previous chapters, I had to create my own language at times to describe how I

\footnotetext{
63 This audio example is played with images from my husband's paintings from a residency in China. We made a video work.

${ }^{64}$ As described in chapter 6
} 
experienced myself, such as "multiclone", "functionoflux", and "ability fluid" (chapter 6).

Agonism forms in the hallway, as the permanent place of conflict between the discourses is accepted in an environment of deep respect and concern for each other: conflict and mutual admiration is present in agonism (Chambers, 2001). In this space the tussle is channeled positively, to recognise and address the ableism that leads to disablement. Foucault uses the term agonistic to refer to the role of struggle within oneself, in order to command one's self.

As the disabled self is often suppressed in society, it is not surprising that I have increasingly drawn on discourses of disabled identities to counter this and give it a space. Indeed, the larger rooms contribute more to this hallway's ambiance than the smaller rooms. But nonetheless, the hallway is a rainbow. A clash of many selves and positions. The music in the hallway is a mashup of all the music from the adjoining rooms. It is also from the hallway that I discover new rooms. New places to inhabit. It's where I contemplate what walls need knocking and what walls are needed to subvert the presence of ableism in my home: A home under constant reconstruction.

\subsection{Summary: A comparison of floor plans, before and after}

If we review the two floor plans, before and after illness/disability and research, there are some major differences to the discourses that I draw on most to understand myself. To summarise, the changes in the rainbow colours of the hallways indicate a move towards positioning predominantly within discourses that identify and attempt to counter the "normate" individual and celebrate difference: accept it. That is, my subjectivity has been reshaped by discourses that help subvert internalised and external sources of ableism. The changes to the hallway rainbow indicate a more agonistic space in which more discourses collide and struggle with each other. My tendency to occupy the hallways the most when addressing ableism indicates the permanent place of the positive aspects of conflict in the politics of ableism. Agonism was the generative site of personal transformation. 


\section{Concluding thoughts}

The exploration above suggests that an agonistic ethic may be fostered in PAMT through increasing the visibility and use of hidden discourses, locating and recognising the normalised subject, allowing a space to be different and disabled, and allowing space for flexibility and change. Both creating the self as a work of art chapters suggest an increased relational ethic, in which agonism plays an important role. Developing PAMT and its ethical foundations has not only influenced the way I understand practice but also personal understandings of myself. The professional and personal were co-constructed in this work of art. 


\section{Chapter 10}

\section{Discussion}

"Layers of different clouds": Photograph from a family holiday

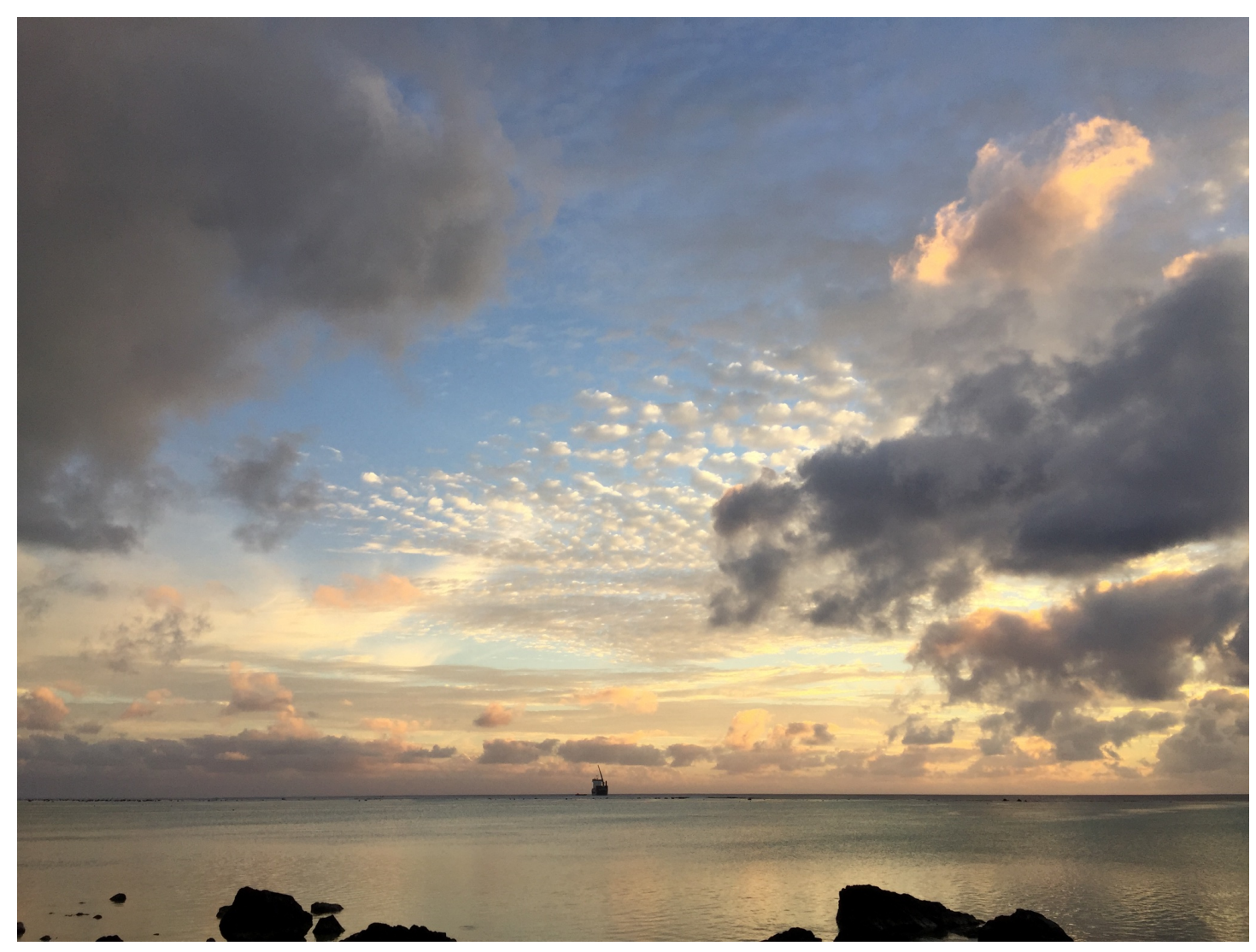

[Photograph of different cloud layers at sunset] 


\section{Introduction}

This chapter draws together the findings of the analysis and creating the self as a work of art chapters to answer the research questions, which structure this chapter. It connects the findings with literature predominantly from music therapy (MT) and disability studies. Furthermore, each research question starts with a piece of music from a disabled musician(s) or disability arts movement that represents or reflects on an aspect of the question findings. This seemed appropriate as the discussion raises the idea of cripping music and making disability studies more visible. I summarise below the most illuminating findings.

This poststructurally-informed, arts-based autoethnography uncovered hidden processes of problematic ableism. This was found within the therapeutic relationship and process examined as well as in the education and professional encounters of a music therapist. Regimes of ableism were supported by universalising and dichotomising narratives. Namely humanism, normativity, observable identifications of disability, and the enforcement of the able/disabled divide through many binaries. The research methodology provided the tools I needed to reposition the practice in order to politicise disability and address ableism. This led to the development of PAMT that extended the relational ethic beyond what was present in my earlier practice. It did so by drawing on aspects of posthumanism (valuing interdependence and deconstructing the human-nonhuman binary), agonistic plurality, and increasing the visibility of disability studies and crip theory. When ableism was uncovered and issues actively addressed through the research process and development of PAMT, my professional and personal narratives as a therapist experiencing illness/disability became more integrated (working with, not against each other in a shared activism). The skills and tools developed through the research methodology enabled me to foster an increased ethical care of the self within practice.

These findings are important because without a dedicated focus on uncovering, understanding and addressing ableism within practice, we continue to constrict and confine clients, therapists and our understanding of what MT is. Research suggests that there is a connection between health outcomes and quality of life with the additional stress of experiencing discrimination (Sue, 2010; Whitehead-Pleaux, 2017a). Sue (2010) discusses four potential ways that microaggressive stressors may 
impact on health: 1) biological (physiological reactions); 2) cognitive (thoughts and beliefs); 3) emotional (increase in intensity of challenging emotions); 4) behavioural (behavioural reactions). People experiencing ableism have negative psychosocial consequences (Green et al., 2005). Ableism is as limiting and is often the foundation and enabler for other prejudice-isms (Wolbring, 2008). Therefore, given its interaction with multiple oppressions, inadequately addressing ableism has consequences for many social problems.

The research highlighted the need to address the almost unconscious acceptance and perpetuation of ableism in a coherent and open way. The findings concur with the notion that the biggest threat and potential harm doesn't necessarily come from those who demonstrate overt discrimination/prejudice, but from well-intentioned people, such as health-care professionals and therapists, who do not knowingly demean or degrade (Sue, 2010; Sue \& Sue, 2015). In the data analysed, such discrimination occurred at a micro-level, in the interactions between people, and was often invisible or subtle. This chapter postulates that the hiddenness and near unconscious acceptance of ableism might, in part, be due to the underdeveloped dialogue between disability studies/crip theory and MT and the embeddedness of humanism in MT. Further discussion of the key findings within each research question and how they relate to literature now follows. 


\section{What different light does a poststructural analysis of the narratives shed on the therapeutic relationship/process?}

Please listen to, "Voices in our Heads" by Johnny Crescendo as the soundtrack for the following discussion: https://www.youtube.com/watch?v=himA42PiaKs. It links with discussions of the disabled people's movement, disabled identities, ableism, and crip music.

Because poststructural analysis focuses on what is not said, thought, or done, it uncovered truths, power effects and ethical issues of which I was unaware or couldn't articulate in my early practice of MT. Consequently, it generated a lot of findings to discuss, warranting substantial discussion of this research question. It uncovered the limitations of the relational ethic in the analysed practice and the need to extend this to address individual and social issues stemming from ableism. I will discuss the three most surprising findings:

- It uncovered the hidden depth of ableism

- It uncovered the productive possibilities of agonism in addressing ableism

- It uncovered problems of normative humanism

\subsection{It uncovered the hidden depth of ableism}

Poststructural analysis revealed that the subtle forms of ableism and normalising practices experienced in my personal narratives were often present in my MT practice. Additionally, it uncovered ableism in the contexts that I worked in as a therapist, in postgraduate education and professional interactions ${ }^{65}$, and across many MT discourses. That is, I uncovered the depth of the role I played in ableism and the contextual shaping of this. I found universalising and dichotomising narratives in the practice studied that supported regimes of ableism: the enforcement of the able/disabled divide through many binaries (client-therapist, biology-culture, humannonhuman, positive emotions-negative emotions, independence-dependence), limited

\footnotetext{
${ }^{65}$ I note that I didn't always encounter ableism and that some work environments, particularly those that fostered inclusive education, were not discriminatory. I appreciated and enjoyed working in these places. However, whilst there was variability in contexts, subtle ableism had a strong presence.
} 
understandings of disability based on observable identifications, success and music based on the normate individual, and normative humanism.

Given that the predominant group of people utilising MT services have a disability or disabling illness, and that ableism impacts on us all, finding the extent of ableism was all the more shocking to me. Processes of ableism had become powerful simply because they were hidden from view. Due to this finding, I don't agree with Humpal's (2017) claim that our MT training, competencies and standards of practice (as they currently stand in New Zealand, 2018) ensure that we are capable of delivering effective music experiences for the culture of disability.

To help understand how ableism became hidden in the practice analysed, I turn to the literature. There have been recent efforts towards theorising ableism in MT, such as within anti-oppressive MT (Baines, 2013), the 'special issue on MT and disability studies' in Voices (v.14, n. 3), the recently published book in 2017 titled, Cultural Intersections in Music Therapy: Music, Health, and the Person, critical thinking papers (Metell \& Stige, 2015) and online training courses such as McFerran's (2018) seminar on critical thinking. Alongside this, more therapists are becoming aware of alternative models of disability to that of the predominant medical model, such as the social model of disability (Gross, 2018). Other critical models supporting social justice of stigmatised identities have also had more presence since my training in 2003-6, such as feminist MT, queer MT (Bain et al., 2016; Boggan et al., 2017), Resource Oriented Music Therapy (ROMT) (Rolvsjord, 2010), writings regarding social justice (Curtis, 2012), Community MT (CoMT) approach to social justice (Vaillancourt, 2012), creative anti-oppressive practices (J. Smith, 2012). Many of these touch on ableism within their approach and some have called for greater inclusion of thinking about ableism. For example, Boggan et al., (2017) suggests that queer MT must more substantially integrate the intersections of ableism and ageism to serve a diverse range of LGBTQ+ clients. The intersection between queer theory and disability studies is growing in awareness.

Despite these efforts, ableism is still under-theorised in MT. For the most part, readyto-use sources in MT theory were lacking in detailed focus on ableism. I propose that this under-theorisation, particularly in New Zealand (NZ) is due to:

- The late arrival of the disability movement 
- Weak engagement with disability studies and crip theory

- The lack of open and direct acknowledgement

I will discuss these three points in order.

The emergence of the disability movement occurred late in the piece in 1990 compared to other liberation movements (Gross, 2018). Interestingly, when Collins first discovered the Matrix of Domination to encompass forms of oppression, ableism was initially left out and was added in a later version (Collins, 1990; WhiteheadPleaux, 2017b). Similarly, it was not a focus in some initial texts, such as in Sue's (2010) phenomenal book entitled Microagressions in Everyday Life. It was, however, included in some of Sue's later work (Sue \& Sue, 2015). Due to this late arrival, disabled activists have called attention to how social movement events are often inaccessible and don't consider disabled people in quests to social justice: an often forgotten intersectional oppression (McRuer, 2006) ${ }^{66}$.

The lack of large scale texts/orientations specifically looking at ableism in MT compared with other forms of intersectional discrimination parallels the late emergence of the disability movement (Gross, 2018). Due to its late arrival, it is not surprising that there are books dedicated to exploring feminism (Hadley, 2006), race (Hadley, 2013b) and post-colonial MT (Hadley \& Cooke, in press), and orientations such as Queer MT (Boggan et al., 2017), multicultural MT (Mahoney, 2015), and feminist MT (Edwards \& Hadley, 2007; Hahna \& Schwantes, 2011; O’Grady \& McFerran, 2006), but no book or orientation dedicated specifically to ableism was found within the confines of the current research ${ }^{67}$. Doing a search on Google, Google Scholar and the Victoria University of Wellington library search engine demonstrated

\footnotetext{
66 "Destroy the Joint", a facilitated community in Australia focused on gender equality, banned some high-profile Australian disability activists from their online page during the \#beingawomen campaign, claiming that the women were in breach of their rules. After posting their experiences of "being a woman", disability activists were deleted for being "repetitive, circular and off topic". Their comments included, "My doctor told me to get a hysterectomy or change my tampon in my office because there was no accessible toilet nearby at my workplace \#beingawomen". This is one example of many that silence disabled women's voices, there are also multiple examples of disabled women struggling to participate in feminism due to a lack of physical accessibility.

${ }^{67}$ As already noted earlier, some critical approaches, such as anti-oppressive MT include all intersectional forms of oppression. I am just indicating here that ableism is still under-theorised comparatively with other forms of oppression in MT.
} 
this lag of the theorisation of ableism. When searching the term "music therapy" with various forms of discrimination one finds that there are comparatively far fewer hits for ableism, the lowest in all searches. The sources it did generate often just mention ableism in passing.

\section{Table 3: Comparison of the number of hits found for terms relating to oppression with "music therapy" on the 27/08/2018}

\begin{tabular}{|l|l|ll}
\hline & $\begin{array}{l}\text { Google } \\
\text { search }\end{array}$ & $\begin{array}{l}\text { Google } \\
\text { Scholar }\end{array}$ & $\begin{array}{l}\text { Victoria } \\
\text { University library } \\
\text { search engine }\end{array}$ \\
\hline "Music therapy" and racism & 383,000 & 2,100 & 851 \\
\hline "Music therapy" and sexism & 389,000 & 870 & 346 \\
\hline "Music therapy" and heterosexualism & 52,300 & 950 & 476 \\
\hline "Music therapy" and ageism & 51,800 & 554 & 186 \\
\hline "Music therapy" and classism & 11,900 & 161 & 92 \\
\hline "Music therapy" and ableism & 9,000 & 148 & 48 \\
\hline
\end{tabular}

As all forms of discrimination are equally important to consider within practice, and they all intersect, there is a need to address any imbalance. We cannot adequately address sexism and racism as music therapists while inadequately addressing ableism and vice versa. Ableism is often an enabler of other forms of oppression, for example, the sexism that stems from women being perceived to lack certain abilities, or when older persons' physical and mental changes become the basis of ageism. Therefore, ableism extends beyond relating to only disabled people and connects with problems facing non-disabled people and societies.

This under-theorisation of ableism in MT could, in part, be due to the underdeveloped dialogue between disability studies and MT to which Tsiris $(2013,2018)$ calls attention. Disability studies is an interdisciplinary field that focuses on disabling social constructions and places positive value on stigmatised disabled bodies (Howe, Jensen-Moulton, Lerner, \& Straus, 2015). It may help dismantle dominant discourses/practices in MT through positional identities that identify and counter problematic ableism.

Despite some steps taken to address the gap, there remains weak engagement between the fields (Tsiris, 2018). Whilst some connections do exist (Baines, 2014; Gross, 
2018; Hadley, 2014; Humpal, 2017; Metell \& Stige, 2015; Tsiris, 2013), for the most part I found the voices of disabled scholars/communities and acknowledgement of political disabled identities to be missing in key books and articles where they would be well placed. Key disability studies music authors such as Straus, Rowden, Lubet, McKay, Stras, and Cameron are not included in many MT texts. They are not referenced in, How Music Helps (Ansdell, 2014), or Defining Music Therapy (Bruscia, 2014), or in some handbooks of MT (Wheeler, 2015). A further example is Aigen's (2014) book which readily discusses community, ecological and musiccentred practices, but lacked a strong presence of disabled scholars, communities and their identities. This is disappointing given that such socio-cultural perspectives in MT have been presented as a way to bridge misunderstandings between disability studies and MT (Tsiris, 2018). I contend that it is difficult for MT to undo negative misconceptions that disability studies scholars may hold, if voices of disabled communities are still missing in such texts.

To further demonstrate how misunderstandings unfold in the literature, I discuss two papers which draw on disability studies, one from a MT perspective (Metell \& Stige, 2015) and one from an ethnomusicology perspective (M. Bakan, 2014). The field of ethnomusicology is tasked with creating and sustaining diverse cultures, such as neuro-diverse musical communities (M. Bakan, 2015), without inciting them to conform, or measuring them against other cultural norms. Bakan's $(2014,2015)$ ethnomusicology work with children with autism spectrum conditions ${ }^{68}$, which he links with disability studies, had a similar approach to some MT orientations in that he cultivated an environment of "free, unstructured improvised music-play in which the staff musicians were tasked to "follow the children's lead"' (p.119, 2014). They were also advised to resist the urge to fix encountered musical problems and play in ways that defy their common-sense musical sensibilities to allow space for the children's music culture. He contends that this work differed to MT in that changes in

${ }^{68}$ Bakan (2015) mentions that autism spectrum conditions are usually referred to as autism spectrum disorders but the latter is less appropriate. Walker (2014) challenges pathologising autism as a "disorder" as to represent it in this way represents a value judgment rather than a fact. The neurodiversity movement sees autism as part of the spectrum of human biodiversity. 
the children's behaviour were not interpreted as therapeutic, as their prior ways of being were fine all along.

However, if we look at this comment in relation to Metell \& Stige's (2015) paper, we see that not all MT orientations try to fix clients and some have socio-cultural objectives. The authors contend that CoMT can create spaces "where variety is the standard and where barriers for participation for children with visual impairment are reduced" (p. 310). My response here is not to list all the MT orientations/papers that do not try to fix clients. I caution that this may instead distance the profession from owning its role in constructing problematic ableism. Instead I offer the question, why does MT continue to be viewed in this (often negative) way in disability studies? Tsiris (2018), suggests that the misunderstanding is partly based on an incorrect assumption that MT is a homogeneous field within which medicalised, deficit approaches to disability are commonly accepted at the expense of acknowledging the multiplicity of socio-cultural orientations. I propose that it goes further than this. It could also be due to the shortfall of MTs recognition of the depths of ableism across orientations and the subtle microaggressions disability study's authors encounter when engaging with MT. The article in discussion by Metell \& Stige (2015) is a case in point. They draw on CoMT and disability studies frameworks to address barriers and draw attention to negative attitudes, yet they titled their paper, Blind spots in Music Therapy. They have negatively associated blind with being unaware, unknowing or ignorant. Outlining the negative attitudes that children with visual impairments face, yet prominently placing a phrase that promotes a negative idea of blindness reduces the impact of the intention to reduce barriers. While the aims of CoMT go some way in challenging ableism, there are still gaps, especially in the awareness and attention to ableist language, revealing superficial conversations about diversity and inclusion.

A more robust dialogue with disability studies might further illuminate the central role of music in embodying and shaping disability and normality, which emerge in mutually influential and parallel ways (Howe et al., 2015). This is important because music was found to be a conduit of disability knowledge and control in my analysis. Disability studies offer a plethora of further examples of how music institutions may construct disabled identities/subjectivities. Straus (2011) draws attention to how the metaphor of music as a human body constructs bodies of work as either normal or 
abnormal. Entire repertoires, such as atonality, may be understood as disabled.

Disability is defined in a myriad of ways within musical institutions (Lubet, 2011). For example, Jazz in the 1920's and 1930's was initially perceived as an active agent of medical and social disorder (a deviation) compared with regular music which was portrayed as having a calming effect on the insane (Stras, 2009). This alludes to the potential idea that "most standard music theories are not only normalising discourses but also disabling discourses" (Straus, 2011, p. 105).

It is from this point that I temper Gross's (2018) view that music-centred MT is one example of a model that is sensitive to the social model of disability. It may be, but the primary focus on music does not automatically mean it is free from barriers or normalisation processes, which the social model draws attention to. I agree, however, that it may provide opportunities for mutuality if the therapist is aware of the power relationships in music-making. Gross's article, is none the less important because it adds to the conversation between disability studies and MT and draws attention to another disability model. Therefore, my caution is not to reduce its application in practice, more so as a reminder of the depths of ableism that can be present across orientations.

The under theorisation of ableism could also be due to the lack of direct and open acknowledgement in some contextual places. In NZ, ableism had not previously been a major focus within conferences. In this context, Daphne Rickson (2014a, 2014b) has described and sought to address disabling barriers within her presentations and work, and I recently added to this trend when invited by the national body to present about disability perspectives at a hui in 2016 (Shaw, 2016). Apart from these examples, however, for the most part, saying the word "ableism" remains the elephant in the room, and anecdotally I note that when I do name it, it is rare that I am met with understanding. It is not a word that is familiar for some colleagues and I have been regularly asked what ableism is when I have used it in discussions with others. While critical approaches are present in the literature, there is a felt lack of actual engagement in approaches to therapy that may name and challenge ableism in NZ. 


\subsection{It uncovered the productive possibilities of agonism in addressing ableism}

The analysis of my narratives shed light on relationships of power and alternative discursive positions of which I was previously unaware, and which produced an agonistic struggle (chapters 8 and 9). Encountering new discourses, meanings and practices opened up a space of increased conflict and tension. It was precisely this space of tension that allowed me to identify and tussle with addressing asymmetrical and negative power relations for people experiencing illness/disability. Therefore, agonistic pluralism ${ }^{69}$ was necessary in developing a political post-ableist theory instead of attempting to reconcile the tension through holding/developing essentialist principles (Cloyes, 2002).

Rather than speaking of an essentialist freedom, Foucault understood freedom and power as co-existing as relations that are observable as "actions upon other actions" (Foucault, 1982, p. 789). Addressing ableism is not to support empowerment for clients, as power is not a thing that one can own. Power exists in relationships in which it can be resisted or engaged and consequently placing the question of who benefits under scrutiny in social justice work. This differs to many social justice orientations proposed in MT that seek to empower clients, such as in Baines, (2013) and Curtis, (2012). Foucault spoke of an agonism in which there is reciprocal incitation and struggle, which isn't a confrontation that paralyses both sides but a productive permanent provocation of power (Foucault, 1982; Gordon, 1991).

Bringing into question power relations, the agonism between them and the binary relations of freedom is a permanent political task (Foucault, 1982). I incorporate Foucault's conception of agonism within the care of the self, Mouffe's (2016) application of agonism to the political and social sphere, and Cloyes' (2002b) insertion of agonism in care ethics (care agonism) (Cornforth \& Claiborne, 2008) in a

${ }^{69}$ Agonistic pluralism is the respectful and productive provocation between discursive ideas/positions and the varying ethics they may hold. Without which the politics of disability, and identifying ableism in practice, becomes limited. 
disabled politics of practice. Within this, the therapeutic relationship becomes a site for agonistic debate and struggle.

Agonism became an important aspect of the extended relational ethic in the development of PAMT. It was a generative site for personal transformation where the ethical care of the self was constructed through contestable and negotiated disability politics. There were many discursive pairs that created tension and provided sites of agonism (generating productive possibilities) in my study, I will discuss three of these in this chapter.

- Agonistic struggle between stable and unstable identities

- Agonistic struggle between normalised accepted music standards and crip music

- Agonistic struggle between posthumanism and humanism

My understandings of ableism were supported by both embodying and often identifying with, disability culture and aesthetics (discourses that reject the normate), and also by challenging the unstableness of disability as a category by deconstructing the disabled-abled divide (poststructural discourses). At various times within my analysis I used each position to highlight different effects of power. Therefore, when discussing ableism, I speak of the importance of both undoing binary-producing identity positions and re-inscribing identity-based reactive responses to oppressive power. In doing so, I recognise that the poststructuralist logic of destabilising identity can free marginalised people from reductionist narratives, but at the same time I understand the risks of denying the particularity of their experiences. "The theoretical bind is that deconstructing oppressive categories can naturalize the effects of real difference" (Garland-Thomson, 1997, p. 23). Agonistic theorists would recognise that politicising disability involves relations of "us" and "them" and relations of identity/difference that can't be resolved (Tambakaki, 2014). This is considered necessary, because when agonistic dynamics of pluralism are hindered because of a lack of forms of identification, forms of politics based around essentialist identities of disability can take hold. A vibrant clash of positions is needed to have agonistic relations that offer possibilities of identification and political alternatives (Mouffe, 2016). 
I realise that for some I am close to the boarders of academic intelligibility by refusing to displace the features of disability cultures and identities whilst undercutting the binary of disabled-nondisabled. But they both held a strong place in the analysis. I sit with the contradiction of knowing the binary is artificial and a product of oppression (Hahna, 2017), but needed when drawing on disability culture that rejects and therefore dismantles the normate. The explanation used by GarlandThomson (1997) drawn from Susan Bordo, that the identity disabled operates as a life-enhancing fiction, is really useful in explaining the findings. Categorising myself as "disabled" at times was a necessary fiction in managing ableism (chapter 9), and abolishing the distinction between disabled-able was necessary in mitigating hierarchies (chapter 6).

In $21^{\text {st }}$ century MT practice, we cannot afford to completely banish the category of disability, as the important lens political disabled identities bring to reducing ableism would be lost. To illustrate this, I use a literature example that sought to reduce the arbitrary divide but instead hid the actual differences and struggles many disabled people face, subsuming difference into the same. Aigen (2014) mentions that musiccentered practice is believed to reduce the abled-disabled divide, as it is based on the idea that music enriches all human lives. However, music may not enrich a disabled person's life if barriers and normalisation processes are embedded in the act of music making. Seeing music as a purely positive resource is both limiting and naïve within the lives of people with disabilities (McFerran, 2017b).

Aigen further states that how people with disabilities engage with music is "no different from how people without disabilities engage with it" (p. 230). This is problematic because it doesn't recognise the many ways of being disabled-abled, with different embodied understandings, strengths and challenges, and it hides the social processes of disablism that impact on how one engages with music. I am concerned that disabled identity politics that are locked into discourses of difference are undermined by such statements (Lather, 2006) and that it may enforce a disabilityblind approach. I liken it to statements of people not seeing race (Hahna, 2017). Whilst it is tempting to think that we can see beyond colour, disability, class and sexuality, the historical and ongoing oppression of persons based upon race, ability etc, cannot be easily erased (Hahna, 2017; Sue \& Sue, 2015). Statements regarding how music transcends disability add to a disability-blind approach. This form of 
denial of difference may hinder rather than help multicultural issues in MT practice (Toppozada, 1995). It also has parallels with queer theorists critiques of the notion of community, that underpins models like CoMT, because it emphasises commonality and superficial inclusion instead of productive differences (Bain et al., 2016).

We need to see and appreciate people's differences to truly value them. Instead of understanding music as bypassing disability to reach the healthy aspects of a person, such as in the notion of the music child (Aigen, 2009; Nordoff \& Robbins, 2007), it's helpful to recognise disabled differences in music engagement. Headlam (2006) described how some autistics hear and conceive music in distinctive ways that differ significantly to individuals who are neurologically neurotypical. Oliver Sacks shares stories of patients who experience seizures that are triggered by music and musical hallucinations in his book Musicophilia (Sacks, 2008). Musicians with physical disabilities have different playing styles that result in unique sounding music (McKay, 2009). As described in chapter 4 and 9, my music making became more interrupted (incomplete compositions) and I preferred music in short snippets when I was unwell. There were times when music did not enrich my life, due to an overwhelmed sympathetic system loaded with three times the average amount of noradrenaline every time I stood, I often needed silence during recovery. This parallels Webster \& Rickson's (2018), suggestion that when unwell, no music might be the right option at times. McFerran (2017) describes how people with mental illness engage in music differently. Furthermore, societal structures impact on engagement, such as noninclusive music groups and financial barriers of access.

Ultimately, it is hoped that the result of this agonism is a valuing of diversity. Showing necessary incompatibilities may highlight the subtle constructions of ableism in MT and provide ways of reconstituting messy and diverse ways of being in practice (Lather, 2006). Thinking in multiplicities, the many different ways of being disabled and non-disabled (as seen in chapter 9), move us beyond simplistic dichotomies towards a more complex understanding of it, of cultural difference based on individual experience "with rich variation of expression" (Hahna, 2017). This agonism that I have described here can be found to be present in crip theory. Whilst crip theory has a contestatory relationship with identity, it does not seek to dematerialise disability identity (McRuer, 2006). It celebrates non-compliance, the re- 
imagining of bodies and desires and futures, and deconstructs concrete thoughts (McRuer, 2006). Therefore, this agonism suggests a cripping ${ }^{70}$ of MT practice.

Relatedly, learning about crip music and disability arts highlighted some of the asymmetric and negative relationships of power within music making. There has been increased understanding that when we are making music together, we are in a cultural and social space that contributes to a process of becoming (Ansdell, 2003). However, it seems that we rarely speak of crip music, disabled aesthetics, and have only recently begun to include the music of disability cultures (Humpal, 2017; Oswanski \& Donnenwerth, 2017). Indeed music therapists are often trained in the privileged Western classical music in academia (Hadley \& Norris, 2016) and normalised Western music standards continue to be used as a primary way to measure success in therapy. For example, Aigen (2014) explains that when an individual can participate in the stylistic aspects of rock music, e.g. maintaining tempo: "there is a circumventing of disability as one is elevated to a higher level of integrated human functioning" (p.54). This statement suggests that if one can meet normalised standards of rock music one is thought to be less disabled which is ideal.

The positional gap of crip music in my prior practice is not surprising given that theoretical links between music and the disabled body have been overlooked in much writing in popular music (McKay, 2013) and musicologies recent arrival to disability studies (Howe et al., 2015). Since the first collection of essays co-edited by Learner and Straus (2006), there has been an increase in interest and writings in this previously neglected topic (McKay, 2013). This literature presents compelling evidence that "pop, rock, and classical music academic critiques are, shall we say, embracing their inner and outer crips" (McKay, 2013a, p. 13; Cameron, 2009; Lubet, 2011; Stras, 2009; Straus, 2006, 2011). The crip music movement counters normative music with alternative aesthetics and engagement in music, due to their different embodiment.

${ }^{70}$ Using the term "crip" or "cripping" is one way for disabled-identified people to take back language that has been used by systems and institutions to harm or demean people with disabilities. 
Furthermore, the representation of disabled ways of being is often not mainstreamed.

For example, some successful disabled musicians have minimised their disabilities or lyrically passed to sustain careers, such as, singing about seeing when one is blind (McKay, 2013). In pop and rock, disabled women are almost invisible (McKay, 2013b; O'Brian, 2002) and few messages in song lyrics depict disabled people in positive ways (Hadley, 2017). Music can become unpopular when it gets too Cripped due to the limits of audience and industry inclusiveness towards the disabled (McKay, 2013). Music videos with disabled people in them are few in the mainstream music industry (Hadley, 2017). Although it is less visible, finding this discursive position of cripping music produced an agonistic relationship with normalised musicmaking/normalised standards, increasing the potential for new possibilities to emerge from the agonistic struggle.

\subsection{It uncovered problems of normative humanism}

The agonistic struggle produced from learning about posthumanism was significant. I will start by outlining the ableist pitfalls of humanism, which were the most surprising finding ${ }^{71}$. Humanistic ableist tendencies of the body and psyche mark those who count or do not count as human ${ }^{72}$. Humanistic ideals of autonomy, control, independence, self-determination, choice, self-actualisation and rationality that create the human subject, also inscribe these characteristics as pertaining to the "good citizen". Such ideals exclude disabled ways of being that demand interdependence, a sharing of autonomy and control and are constraining due to being unobtainable and at odds. They produce disability as "other": (ir)rational, (non)autonomous,

\footnotetext{
${ }^{71}$ Some colleagues have mentioned that they do not see that their work is an issue for disabled people, as they do not work in a medical model. Other models too have ableism deeply hidden as described in the analysis.

${ }^{72}$ Humanism has been described in many ways throughout its history (Abrams, 2018). It is difficult to define due to the multiplicity and vagueness of the concept. The main principles are human uniqueness, the importance of individual identity, principles of logic/rationality and pursuit of human virtue ("'Humansim,"” 2019). Humanism attaches prime importance to the human and centred on the notion of the rational, autonomous self. It seeks out organic unity in the human body. Davies (1991) describes how within this discourse, agency is synonymous with being a person. Within humanism conscious rational linguistic processes are used by individuals to dominate those irrational, emotional aspects of self that might otherwise disrupt claims to coherent adult identity.
} 
(non)competent and dependent. Disability in the context of humanism stands for a "self-gone out of control, individualism run rampant: it mocks the notion of the body as compliant instrument of the limitless will and appears in the cultural imagination as ungovernable, recalcitrant, flaunting its difference as if to refute the fantasy of sameness implicit in the notion of equality" (Garland-Thomson, 1997, p. 43). In short disabled people are excluded and struggle to be considered fully human within this discourse whilst other human bodies are validated and affirmed.

Problematic humanistic shaping of the human was found through the goals and successes pursued and set in the studied practice. Therapeutic goals were previously, albeit unknowingly, shaped by the requirement of a compliant and regulated body, necessary for self-government and self-determination (Garland-Thomson, 1997). Thus, potentially placing pressure on individuals, from the idea of self-determination, to feel responsible for their own economic, social, and relationship successes and failures (Garland-Thomson, 1997). Humanism restricted my ideas about therapeutic success and unwittingly positioned disabled people as failures when sessions did not enhance humanistic ideals. Other ways of being, such as passive, interdependent, still, limited, in an uncontrollable body were subjugated when autonomy, control, independence and self-determination were only pursued. Furthermore, the myth of wholeness could not be maintained by the imagined autonomous individual that has safe boundaries enabling unrestricted self-determination (Garland-Thomson, 1997). The down sides of humanistic ideals, such as individualism breeding selfcenteredness and self-determination turning to arrogance and domination (Braidotti, 2013), lacked consideration. Ultimately, the humanistic shaping of the human in therapy can contribute to the othering of disabled people and them being seen as a threatening presence that generates other's anxieties about loss of control and autonomy.

However, I acknowledge that there were some helpful aspects of humanism, in particular being warm, its focus on the present moment, and civil rights based upon definitions of humanity and normative legal systems that respect the right to live as a human being (Braidotti, 2013). Like Goodley, Lawthom, \& Runswick Cole, (2014) I am not suggesting we give up on the rights that are attached to the individual nor the social justice of equality in our fight to challenge disableism, especially given that the 
disability rights movement has been vital in the acceptance and recognition of disabled individuals as human and disability culture (Humpal, 2017). But I later propose another way of thinking about human rights, because as Braidotti (2013) suggests, it is ethically and intellectually impossible to disengage the positive elements of humanism from their problematic counterparts. In particular its relationship with colonizing and ableist tendencies of the body and psyche (Goodley et al., 2014).

To further understand the construction and problems of normative humanism in the practice studied, I will now discuss:

- Humanism in MT

- The different light of posthumanism

- The impact of the humanistic grand narrative on agonistic pluralism

My analysis shed light on how humanism and its ableist tendencies had become naturalised and normalised in the practice studied. This normalisation of humanism correlates with wider thinking in MT. Aigen (2014) states that in actual practice, a substantial number of music therapists combine elements of psychodynamic and humanistic thinking..."a humanistic approach where the relationship is based upon a genuine encounter between two authentic individuals provides a natural fit for what naturally happens in music therapy sessions" (p. 111). My research illuminated that humanism is not always a natural fit for disabled people and consequently MT.

As Ansdell and Stige (2018) outline, humanism has a long history in MT and has been present since the 1960s and 1970s. Humanistic perspectives have been thought to be useful in developing the identity of the MT profession (Ruud, 2010) and indeed it has played a major role. Aigen's (2014) third stage of MT development (1982present) contends that many of these generic theories are based on a humanistic value system. This is affirmed by Ansdell and Stige (2018) who contend that in contemporary MT the humanistic tradition is solidly present. It has even been argued that "every form and expression of music therapy is, in some sense, humanistic. Music therapy, in all of its various manifestations, is informed by a fundamental relationship to other persons, within humanity" (Abrams, 2018, p. 1). 
Consequently, humanistic goals, such as self-actualization/creativity, competence, autonomy, and wholeness, are prominent in the literature. Important to humanism is the concept of wholeness where humans strive for integration of the self. MT journeys towards wholeness have been thought to be powerful and transforming (Aigen, 2005; Kenny, 2006; Viega, 2013). Humanistic ideals of independence and autonomy have also been believed to be essential for maintaining "a sense of preferred identity for people living with illness who are faced with increasing dependence" (Magee, 2017, p. 3). Music-making here is thought to challenge disabled people's self-constructs of dependency "leading to a sense of "wholeness" of self" (Magee, 2017, p. 3). In another paper, Magee (2011) suggests that assisting the independence of people with complex needs in MT is an important factor that contributes to quality of life.

Disability scholars have questioned the relationship between independence and a good life, focusing instead on the quality of interdependent relationships that promote quality of life. These are just some examples of the shaping of the humanist subject in MT which subjugate the interdependence that disability demands. The importance placed on humanistic ideals in MT practice is not surprising given that many ablebodied/minded people cling to an ideology of autonomy (Howe et al., 2015).

Because humanism with its focus on independence and centrality of human agents limited the relational ethic of my practice, I turned to Braidotti's posthumanism to extend relationality. The posthuman subject, and its music, is an assemblage of human and non-human, planetary and cosmic, given and manufactured entities. It opens itself up as an extended, distributed, interconnected and relational being that is embodied and embedded (Braidotti, 2013). Hence, it moves beyond the boundaries of the of human - non-human (chapter 6) and dependent-independent dichotomies (chapter 7).

The interdependency of the posthuman subject is in accordance with critical disability studies major theme that "disability necessarily demands and affirms interdependent connections with other humans, technologies, non-human entities, communication streams and people and non-peopled networks" (Goodley et al., 2014, p. 348). In fact, the posthuman subject is disabled as we all rely and are dependent on these multiple relationships. Of importance to disability studies is the lesson that dependence is not a defining element of disability status, "rather interdependence is perhaps the defining feature of civilization" (Lubet, 2011, p. 6). "Mutual dependence, care, and assistance" 
bind us all together (Howe et al., 2015, p. 8). I rely on the pilot to fly me to another destination, the farmer to grow my vegetables, my neighbours to empty my letter box while I'm away, to name a few examples. Therefore, like disability studies, the posthuman subject poses the question whether anyone is in fact immune to external forces, independent of physical limitations and not in need of care and assistance from others. The illusion of the self-governing autonomous individual, and therefore the fantasy of able-bodiedness, is exposed in posthumanism. Disability captures the productive possibilities of the posthuman condition (Goodley et al., 2014) and demands to be recognised not as lack, but possibility: an enhanced extended humanity.

As I became aware of the need for a new category of identity based on the partial, incomplete subject whose realisation is dependency and interdependence not independence and autonomy (L. Davis, 2013a), I created the position of PAMT. This departed from the humanism in contemporary practice towards a strong association and conscious application of aspects of posthumanism. There is potential for posthumanism and PAMT to be at ease with each other because disability has always contravened the traditional conception of what it means to be human (Goodley et al., 2014). For a therapist to occupy a posthuman position, disengagement from the dominant normative vision of the self is required in the process of becoming relational in a complex manner (Braidotti, 2013; Goodley et al., 2014). PAMT supports such complex connections and collective support. As one of the aims of PAMT encourages therapists to question their own frame of reference, there is a heightened sense of relational ethics that urges us to think about how we are all made through our connections with others. Success from a Braidotti posthumanist perspective when applied to MT would be marked by positive possibilities and connections, relationality, and disruptions/surprises that make us think differently. Such markers are supported by the PAMT framework.

MT critiques of humanism have not lessened its dominance. The practice studied also demonstrated the pitfalls of the traditional medical model in practice. Such critiques have been present since the 1960's (Aigen, 2014). Indeed, Rolvsjord's (2010) critique of the medical model is comprehensive and far reaching. However, unlike the comparatively small amount of critiques of humanism in the MT literature, medical 
model critiques have sparked great changes to be made to practice. Orientations, such as CoMT, field of play, and ROMT, have developed in response and reposition practice away from the medical model. From and within the critiques of humanism, however, there is still the desire to "hang on" to humanism as a dominant framework in contemporary practice at the cost of developing more pluralistic foundations and diverse positions. The consequence of which is that the grand narrative of humanism stifles agonistic pluralism.

On the $5^{\text {th }}$ of July 2018, I presented a paper on posthumanism in MT practice at "Cripping the Muse", Leeds University. The following day ( $6^{\text {th }}$ of July) Music Therapy Perspectives published a special issue looking at humanism in music therapy practice. Ansdell and Stige (2018) mentioned that to their knowledge there are no substantial posthuman critiques within MT. This discussion, and the use of posthumanism in PAMT, is therefore timely. To demonstrate the professions current desire to hang on to humanistic foundations, I draw on this recent issue as an example.

The Music Therapy Perspectives (2018) issue on humanism was published due to the concern that the MT field had yet to establish its own definitions and parameters on the subject of humanism. Excitedly I read as the parameters of humanism were pushed with Hadley and Thomas's (2018) critical evaluation of conventional humanism and Ansdell and Stige's (2018) posthuman critique of humanism. However, I was left feeling underwhelmed by both articles: they concluded with returning to humanism in an extended critical form.

To explain my reaction I will further discuss both articles. Hadley \& Thomas, (2018) and Ansdell \& Stige, (2018) are notable for critiquing conventional humanism's exclusion of some groups, its individualistic focus, and focus on independence. They call for an extension/critical expansion of humanism that will address these issues. However, based on the findings of my research, the expansions proposed do not go far enough in addressing the power relations that create problematic ableism. Whilst they do caution that the article is just a starting point for continued discussion, Hadley and Thomas (2018) did not address a binary formed under humanism that is constructed by and constructs ableism, that of the human-nonhuman binary divide. The ontological divide between humans and non-humans has arguably been the 
deepest and most invisible hierarchy on which racist and sexist hierarchies, for example, have been grounded (Lennard \& Wolfe, 2017). The racism inherent in black people being called apes/monkeys relies on the idea that they are not human. Ableism sustains this divide and therefore we cannot adequately address social injustice without addressing it.

Whilst Ansdell and Stige (2018) do acknowledge the hierarchy of being and gap between human beings and non-human entities in humanism, they were perhaps unwilling to fully allow posthumanism a foundational place in MT practice. Instead they strongly proposed an extended humanism as the future conceptual spine for MT and denied posthumanism a proper seat at the table. It was hard to not feel as though the extended humanistic foundation proposed was just a wolf in sheep's clothing due to the felt message of: "we've heard your complaints, we will make interior changes, but we will continue to address your complaints within the structure that generated them in the first place-no other forum is needed". Addressing ableism is more complex than simply incorporating disability issues into existing dominant frameworks that have historically been hostile to disabled people.

Ansdell and Stige (2018) considered posthumanism to be too radical. They present a posthuman move of MT as the erasure of the humanistic framework, "creating a radical discontinuity of the tradition" (p. 6-7). I do not see its inclusion as a foundation for practice to be radical or discontinuous, particularly in a NZ context. One can find many hints of posthumanism in anything that decenters the human in relation to the world, such as in indigenous theories of practice. There are aspects of it in Māori models of health practiced in MT where there is a strong presence and appreciation of the interrelatedness with non-human entities/environments (Hodgson, 2014). Further abroad, in her indigenous first nation MT practice, Kenny consistently advocated for a practice that involved our connection with the environment. Some definitions of MT include the role of non-human entities within therapeutic relationships (Bruscia, 2014). Ansdell and Stige (2018) themselves acknowledge aspects of posthuman thought in ecological and cultural models of therapy. Given this, embracing posthumanism as another foundation for practice would not be radical but instead support practices that are already occurring, whilst also allowing stronger agonistic pluralism. If we strive for humanistic continuity in the tradition of MT, as they suggest, we will continue to reduce the provocation engendered from alternative 
discursive foundations that can produce productive possibilities that move us beyond dominant approaches and reach beyond the normal range of ethical thinking. It is not just a mode of critique, it has much potential as a position from which to practice and in generating greater agonistic provocation when given a more prominent place in practice.

There was a concern about losing the idea of personhood that incorporates certain rights if posthumanism serves as the foundation of practice. However, human rights and liberties from humanism and posthumanism are not mutually exclusive projects. Although posthumanism doesn't base disabled people's rights on a moral standing based on diminished versions of the normate human, it values all forms of life not because of its second class form of the human but because the diversity of life is to be valued, in its difference and uniqueness (Lennard \& Wolfe, 2017). It is actually an issue of context and location that makes humanistic discourse a requirement at times. For example, when petitioning to parliament for accessibility, one needs to speak the language of humanism, as legal and political structures are consolidated in humanism. This is not to say that unequal power relations and rights cannot be addressed through posthuman thought itself. It is helpful to bear in mind that posthumanism doesn't mean anti-humanism (Lennard \& Wolfe, 2017). I recognise that humanism provides an important legacy in MT but found that it struggled to describe the complex ways of relating in therapy and profound interdependence experienced in some disabled ways of being.

While the idea of expanding humanism is not new to MT practice, it seems that no theorist has fully embraced posthumanism as an active foundation for MT practice. My research takes that necessary step in claiming posthumanism as an important foundation of PAMT. This has not been about abandoning humanism but developing the ability to re-position and move between different paradigms when needed. It will further allow the appreciation of many kinds of abilities, successes, and ways of being. 


\section{How did the close examination of a music therapist's experience of illness/disability impact on their professional practice?}

Please listen to the performance of Coldplay's "Strawberry Swing" by The British Paraorchestra as the soundtrack for the following discussion: https://www.youtube.com/watch?v=Pu9CvbGJmss. It may provide helpful reflection around the performance discussion, as well as demonstrating the importance of music for the sake of music-making.

The close examination of my experience of illness/disability impacted on my practice by making me more aware of different illness/disability related ethical issues. As noted in the above question, it shed light on processes of ableism within my practice and new alternative positions from which to practice. As I became increasingly aware of these during the research process, my practice changed. Ethical care actions occurred alongside the research, such as working more collaboratively (co-writing reports, assessments, plans with students), taking a disability activist role, becoming less goal-focused (where possible), changing language that othered, pathologised or marginalised the people I worked with, amongst other actions taken in the wider context of MT in NZ (chapter 8).

The close examination of my own experiences helped me to reposition my practice to address hidden forms of ableism. The examination led to identifying the need to extend the relational ethic in my practice, primarily through posthumanism's valuing of interdependence, mutuality/non-hierarchical being, diversity, creativity and beauty, and also encompassing agonism, subjectivity, and negative capability. This developed into a practice I called PAMT. Where, PAMT differs from other MT theoretical orientations is its departure from the foundation of humanism, the inclusion of agonistic plurality, and perhaps its close relationship and alliance with disability studies/crip theory. As this has already been discussed above, I now primarily focus on the ways in which the new position of practice connects and dialogues with other MT approaches/theorists to further answer the question. My practice was impacted in the following ways: 
- It increased the prominence of music

- It increased performance opportunities

- It enhanced connection with culture/context

- It directed attention to social justice and critical issues

\subsection{It increased the prominence of music}

In my development towards PAMT, music took on a more prominent role. Like Aigen (2014), I became aware that the use of music to achieve non-musical ends was sorely lacking and increased the role of music for the sake of music-making. The definition I used as a new graduate that, "MT is the use of music to achieve non-musical goals" was inadequate. The restriction to non-musical rehabilitative goals disconnected disability issues that occurred from social and cultural contexts. For example, disabled people can struggle to access music making activities. Sometimes the only access is in the form of MT, which in such cases may restrict disabled people's music to only being meaningful and possible if in pursuit of non-musical goals. PAMT supports the idea that MT can be justified "on the basis that it provides music to many marginalised individuals" (Aigen, 2014, p. 70). Including musical goals attempts to counter the narrative that disabled people must access music through non-musical criteria as opposed to able bodied/minded people who access music for music (Aigen, 2014).

Increasing the prominence of music also increases opportunities for creativity and beauty. Many MT approaches highlight the place and importance of these aspects, such as creative music therapy (Nordoff \& Robbins, 2007), field of play (Kenny, 2014), indigenous understandings (Hodgson, 2014) and aesthetic MT (C. Lee, 2003). As discussed in chapter 7, such oppporuntities became even more important when unwell. Hence, PAMT incorporates these aspects within its ethic, which is supported by posthumanisms commitment to creativity.

Various MT perspectives support the notion that a deficit of music in one's life is a legitimate reason to come to MT (Aigen, 2005). For example, in aesthetic MT, CoMT, and music-centered MT, human motivation for musical experience is a valid self-justification for MT. Verbal interpretation of the experience does not need to take place for the therapy to be considered valuable in music-centered practice (Aigen, 
2014). PAMT supports this, as demonstrated in vignette of Tallulah. Music-centered practices also do not pathologise people according to their diagnosis, such as treating schizophrenia. Likewise, instead of pathologising, the practice of PAMT places importance on recognising the ways in which disabled people themselves define their lives and experiences, disabled aesthetics and various disabled musical identities. Understanding that musical engagement with another person can construct and impact on disabled identities.

\subsection{It increased performance opportunities}

One prominent debate in MT is the use of performing and recording. CoMT supports the use of performing whilst other orientations view this as controversial. Within PAMT performance is seen as a potential way for disabled people to share their music and extend what it means to make music. Disabled peoples' music need not only be private and may, when performed, have the ability to challenge public perception and engender appreciation (M. Bakan, 2015). However, it is imperative that the players and therapist assess this to be potentially beneficial before the performance occurs. A potential detraction to performing could be performers being overpraised or labelled as inspirational for simply performing music. There is the potential for such performances to be used as inspiration porn ${ }^{73}$. Rickson (2014c) notes that support people who assisted students with disabilities to perform with a professional orchestra drew on overcoming and vulnerability narratives to describe the performance experience. Although performing differences that are stigmatised can mark one as a target, it also exposes and resists the prejudices of society (Siebers, 2008). This contradiction always needs consideration.

Greater discussion of the role performance may play in inspiration porn and ways to manage this would be helpful going forward. It raises questions such as, are the social contexts performances take place in able to accept disabled people's music in nonbelittling and non-oppressive ways? How does the MT process help create safe spaces for performance? The performance programme, for example, might be a way to

\footnotetext{
${ }^{73}$ Inspiration porn is a term coined by the disability activist Stella Young in 2012 and refers to the portrayal of disabled people as inspirational on the basis on their disability. The word "porn" is deliberately used to allude to the entertainment and warm fuzzies non-disabled people get from the portrayal of the inspirational disabled person.
} 
outline the ethos of the performance and use inclusive language. The need for music standards to be adapted is acknowledged in the literature, but how do we balance this with audience expectations?

One potential way of managing performance related issues would be to seek the advice from disabled communities. For example, after Bakan's (2015) neurodiverse group Artism performed at a conference, he was given the following feedback by the Autism community. Suggestions included: decreasing the loudness of the performance due to the sensory challenges that many Autistic people have; briefing the audience to show appreciation not by clapping, which may disturb sound sensitive Autistics, and instead use Jazz hands; do not refer to autism awareness as this phrase is associated with Autism Speaks and other such organisations that are not run by Autistic people. Their projects of awareness are often offensive within the Autism self-advocacy movement that instead promotes autism acceptance. In summary, performance opportunities are important for everyone regardless of ability/disability, but there is still work to be done in creating less disabling performance environments.

\subsection{It enhanced connection with culture and context}

"Many newer conceptualisations of the therapists role relate to an enhanced awareness of community and cultural contexts" (Aigen, 2014, p. 31). As my practice developed alongside the autoethnography my awareness of culture and context also increased and became an important aspect of PAMT. This brings into question, is PAMT and CoMT the same thing?

PAMT shares many overlaps with CoMT. Some characteristics of CoMT that Stige (Stige, 2002) cites are applicable to PAMT: the community is not only a context for the work but also a context to work with, social and cultural isolation is considered a potential "health threat", involves other contexts outside the MT room and other people, more public and political than conventional MT. Aspects of Ansdell's (2002) theoretical framework for CoMT also apply to PAMT. For example, working with clients from cultures without a concept of therapy, working with a central need for connection, addressing needs of individuals not formally identified as clients (parents, teachers), the incorporation of performing or creating recordings. 
However, PAMT extends the relational ethic of CoMT further with posthumanism, which is a shift from the humanistic thinking still present in CoMT. I note there are signs of movement, for example, the CoMT literature thinks of autonomy as a relational accomplishment, much like the shared autonomy I propose. However, much literature still privileges, and puts at the centre of the practice, human connections with only some mentioning the physical environment. For example, Hoskyns' (2016) review of Ansdell's (2014) book, noted that relationships with the natural world, the landscape and place of experience were missing. A humanocentrism often results creating boundaries around the human subject. Therefore, some CoMT literature continues to restrict and confine understandings of the human with universalising narratives. For example, Aarø \& Stige (2012) write "universal human capacities make musical community and collaborative musicking possible" (p.26) ${ }^{74}$. Therefore, CoMT and PAMT have different ethical and theoretical foundations. Further subtle differences can be noted in some of the seven qualities of CoMT proposed by Aarø \& Stige (2012), particularly the qualities of resource orientated (strength orientated therapy), reflective therapy, and ethics based on valuing human rights. PAMT sees weakness and limitations as possible resources, and dismantles the binary of positive and negative emotions I encountered in a resource orientated approach, and ethics are based on valuing all forms of life (human and non-human entities). As previously noted, multiple examples of ableism were found to be present in the CoMT literature, which suggests that there are gaps in CoMT that requires the alternative lens of PAMT.

\subsection{It directed attention to social justice and critical issues}

The research process led towards my identifying with and developing more understanding of critical perspectives, which were lacking in my early training (20042006). The current research demonstrated that understanding disability primarily from the medical model is an outmoded restrictive perspective. In this regard, I have followed a current trend in moving from my understanding of the medical model towards greater inclusion of social/cultural models. Ten of the thirteen contemporary

\footnotetext{
${ }^{74}$ As previously contended, the hierarchical divide between the human and nonhuman is both a result and contributor in the perpetuation of ableism. We must address this divide and the centralisation of the human to increase the appreciation of many diverse ways of being.
} 
orientations outlined in Aigen (2014) critique the medical model as a foundation for MT practice and six consider the social context. Whilst PAMT incorporates a range of definitions of disability (due to its heterogeneity and intersectionality), the medical model is decentred with the inclusion of cultural, affirmative, minority, and biopsychosocial understandings.

Many music therapists are grappling with critical issues in their practice (McFerran, 2018) and ways to promote social justice. Indeed, there has been an increase in critical approaches. Findings from my research connect and resonate with this literature. Like Rolvsjord's ROMT practice, PAMT values collaborative relationships and mutuality to address imbalanced power relations. I also connect with her call to move attention "away from the therapist and the music's capabilities and toward how clients make use of music and music therapy" (Rolvsjord, 2010, p. 52). This was important because I did not wish to repeat the same processes of voicelessness I experienced as a patient with the people I was working with. Hence, co-collaborating with students (where appropriate and possible) in assessments, plans, and reports became integral to my practice. I also note the possibilities for collaborating in research and publications, as Fairchild \& Mraz (2018) and Rickson (2014d) demonstrate. Like Sears (2007) the belief that a MT theory should be non-hierarchical and non-linear is upheld in PAMT. Queer MT, drawing from queer theory, challenges the concept of normal and fixed identity (Boggan et al., 2017). Likewise, as demonstrated in chapter 9, PAMT emphasises a fluid, discontinuous, incomplete self and posits the need to let go of quests to find ourselves and others and instead appreciate many selves to support inclusivity and diversity.

Feminist MT, post-colonial MT, CoMT, Queer MT, and anti-oppressive MT, all call for the politics of social change to be a necessary focus for therapists. Otherwise therapists are just helping people to adapt to a dysfunctional culture (Aigen, 2014). Such theories really informed and impacted on my practice as they helped me to address some of the ethical concerns my personal narratives raised in regard to my work. This again may propose, for example, the question: is there a need for PAMT that looks specifically at ableism if there are already MT approaches that address social justice and if the entirety of an individuals' experience may not be understood by focusing on only one form of oppression? My research suggests that there is. 
Whilst no forms of oppression are worse than others as they are all interconnected and damaging to people and societies (Oswanski \& Donnenwerth, 2017), different forms of oppression can have similarities and differences in practice that are helpful to understand. Furthermore, ableism has been slow in entering the MT professions' consciousness, as previously discussed. Therefore I offer PAMT as a lens to be applied to, and in conjunction with, other approaches precisely because we need to see the struggles of all oppressed groups (Whitehead-Pleaux, 2017a) and understand individuals through an intersectional lens. PAMT offers the valuable lenses of posthumanism and agonism in the work of social transformation.

\section{How can the work of therapy be managed when client and practitioner-patient share similar illness/disability narratives?}

Please listen to "Going Nowhere" by Prudence Mabhena and Liyana as the soundtrack for this following discussion:

https://www.youtube.com/watch?v=MBAlE0bKx2A. The lyrics capture the feeling of going nowhere and the struggle for everything when individualistic management strategies are solely sought.

Before answering this question, I would like to re-examine the way this question has been framed. My question initially implied that the therapist must "manage" the situation and therefore it has an individualistic focus. My research demonstrated that some work cultures, societal norms, ethical codes, education policies, and standards of practice all contribute to the challenges of disabled practitioners/PPs in NZ. For example, NZ ethical codes in MT mark illness/disability as a potentially dangerous state in which to practice, creating othering instead of inclusivity as one of the many human experiences that can have implications for practice. An extended understanding of disability beyond the medical model would place the "management" of the position in a different place: away from individual to the social.

Therefore, it is actually not just the therapist's role to manage the situation, nor should they have to attempt this by adapting to a dysfunctional system, with unjust processes. Failure to address and understand systemic factors results in harmful practices for both client and therapist with similar narratives. Changes on a social and cultural level would significantly reduce the need for the therapist to manage. Therefore, my 
research discovered that the position of sharing similar illness/disability narratives was best managed for me when I actively sought to address inequalities and ableism. Hindsight is illuminating and a more appropriate research question might have been, What barriers does a music therapist experiencing illness/disability encounter in practice and within the profession?

There is scant MT literature that would help to answer such a question. However, Hadley (2017), draws attention to some microagressions disabled students/therapists face: Students being told that they are exploiting their disabilities to their advantage; being complimented when needed accommodations are not used; potential students kept out of the profession because their impairments have meant that certain specific competencies could not be performed; no adaptations made to accommodate disabled students; and a boss repeatedly teasing a therapist for frequent misspellings with known dyslexia. Whilst Bybee (2017) researched the occupational oppression of MT in her Master's thesis, she didn't address ableism within the profession (it was perhaps beyond the scope). This could be an area for future research.

A driving force of this research was to bring into relief the fundamental ambivalence I felt as one who had previously shaped a practice based on specific meanings of illness/disability, and some of the internal conflicts that challenged them from my personal experience of illness/disability. These were resolved (partially) by a care of the self that actively challenged ableism within practice. When disability issues were acknowledged, and action taken through PAMT, my position as a practitioner-patient was more easily managed. This was because my professional and personal narratives became more integrated (working with, not against each other). For example, when an ethic of mutuality was fostered a more balanced power dynamic ensued. This allowed disability a place in my subjectivity and was not suppressed in favour of maintaining the expert role. We had equal status. In another example, limited therapeutic understandings of success made me feel failure both professionally and personally but questioning and extending understandings of therapeutic success created a more integrated positive sense of myself (chapter 7). Whilst these examples point to more integration and congruence, I note that I do not mean an absence of tension between the narratives (the place of agonism). More so, that they were congruent in their aims to find less restrictive spaces for clients and myself. I worked against incongruences, 
such as challenging structures and power relations that negatively impacted me in my personal life, whilst engaging these in my practice.

It was important for me to pay attention to incongruences, as there were moments prior to and during the research process where I felt I could no longer be a music therapist because the values drawn from my disabled experience seemed incompatible with the political and educational structures which surrounded and framed my practice. On reflection, the disturbance I noted in the thesis introduction likely occurred from the growing awareness of ableism within my practice that was brought about by my own encounters with it. As Sue (2010) contends, as microaggressive perpetrators become increasingly aware of their biases they often experience debilitating emotional turmoil and cognitive disorientation. Additionally, my description of something feeling not right is a common experience for those who experience subtle microaggresions (Sue \& Sue, 2015). In some ways, I was working an answer in that very first journal entry. By repositioning my practice, I no longer had to try and make my client's, or my own, flower whole, we were already the beautiful aesthetic of atonal music and the interesting shapes made from the holes of insects' lunches (chapter 1). We just needed a space in the garden to flourish in our own way. When such mutual diversity was valued in the therapeutic process, there was more space for a therapist experiencing illness/disability.

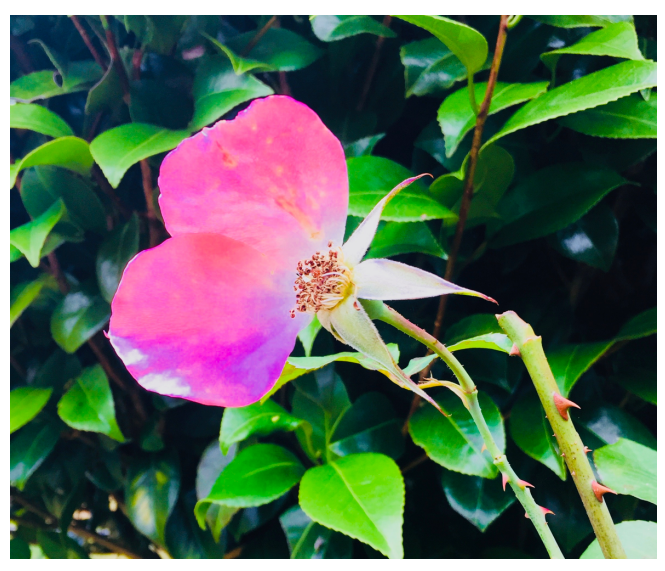

[Photograph of a pink rose with missing petals] 


\section{What does the method/writing of a poststructurally- informed art-based autoethnography offer one's practice?}

Please listen to "Both Sides Now" by Joni Mitchell as the soundtrack for the following discussion: https://www.youtube.com/watch?v=4NdsnFZm0X4. The lyrics capture a reflexive process (looking at both sides and moving beyond them), the feeling and place of not knowing, and change of the self that results, which links with ideas expressed below.

The method of a poststructurally-informed, art-based autoethnography fostered an increased ethical care of the self within my MT practice. Ethical care of the self is concerned with the ways one is incited or invited to recognise moral obligations, and the ways in which one changes in order to become an ethical subject (Packer, 2011). The most significant change generated from the methodology was the creation of a new position from which an extended relational ethic could inform my practice. There were many aspects of the methodology that fostered an increased ethical care of the self through:

- Fostering reflexivity

- Fostering subjectivity as an historical textual construction

- Fostering creativity

- Fostering awareness of the contextual space of therapy

- Fostering support from the literature

The increase in ethical care of the self relied on enhancing key skills that are paramount to MT practice, such as reflexivity, creativity and awareness of the contextual space of therapy. As postulated from the multidisciplinary literature reviewed in the methodology, autoethnography is believed to support these skills. Instead of re-reviewing this affirming literature, I describe how autoethnography enhanced these skills during my research process and the importance of this. I start with reflexivity as this was facilitated through three of the proceeding aspects discussed: Subjectivity as historical textual construction, creativity, and awareness of the contextual space of therapy. 


\subsection{Fostering reflexivity}

During the autoethnographic process I experienced an increased reflexivity. The pace by which my previously held ideas were changing became more rapid through the reflexive process of examining my values, assumptions, prejudices, theories, and attitudes. I found incongruences in my actions with my new espoused values and theories. For example, in the vignette of Callum I recognised my role in trying to restore a normal self (as it is currently constituted). Due to critiquing normalisation and valuing diversity I changed my actions to align with this, following Callum's desire to work alone. Another example was reframing Marcus's sessions with posthumanism to align with valuing interdependence. Further examples were found in chapter 8 of a reflexive process that recognised my active role in shaping my surroundings and ways of relating. The reflexive process moved the mutual practice with participants beyond the experience of being together to that of becoming together (Ansdell, 1995). This increased reflexivity was facilitated through the three proceeding aspects, as reflexivity requires an awareness of cultural, social, personal and discursive contexts in which we live and work, awareness of our contextual subjectivities, and creativity to change.

\subsection{Fostering subjectivity as an historical textual construction}

Looking at my personal narratives through the lens of poststructuralism, with its understanding of subjectivity as textual constructions, facilitated the increase in the ethical care of the self. As Kamler (2001) describes, writing the personal transformed my subjectivity and consequently my practice. However, it was from Foucauldian analysis that I was able to recognise my personal experiences as discursively produced and therefore changeable. Foucault described how literature of the self cannot be understood without uncovering and considering practices of the self (Foucault, 1984a). Without targeted tools, practices of the self are challenging to analyse because techniques of the self are often invisible and are frequently linked to supporting others to manage themselves (Foucault, 1984a). My research affirmed his ideas, as using Foucauldian tools allowed me to come to know and confront my silences. As Kamler (2001) describes, this, for me, was more transformative than the understandings of writing as "therapeutic", "empowering”, or "emancipating": although there are many connections between transformation and therapy. For 
example, whilst I was transformed by the process of reconstructing my practice and subjectivity, I do admit that trying to understand new theory and new discourses was in part bound up with discovering another self that could be more fully accepted.

This relates to positioning theory where people use words to locate themselves and others (Davies \& Harré, 1990). Literature on positioning theory includes intrapositioning in which a person engages in private moral reflections through positioning issues between members of small groups in intimate interaction (Harré, 2012). Self-narratives are viewed as a device for positioning oneself (Harré, 2012). The autoethnographic process that included Foucault's critical ontology of ourselves, was a powerful tool in co-constructing the self and practice through exploring and finding different discursive positions and subjectivities. I started out describing myself as a practitioner-patient (in the introduction and literature review). I then alluded to claiming a positive disabled identity for myself at various research stages, and also created the identity of being Ability Fluid. Similarly, from a professional stance, I began as predominantly a humanistic therapist with (unknowingly) medicalised understandings of disability, to a therapist who re-positioned her practice through developing PAMT. Whilst I don't describe this transformative aspect as therapeutic, I do believe it eliminated some of the need for therapy ${ }^{75}$. I was able to see myself as a historical subject, rather than a defective one.

\subsection{Fostering creativity}

Creativity was a critical part of the ethical care of the self. Being creative through arts practices allowed me to go beyond previous limits and create new thoughts and practices. For example:

- The improvisation process distilled the three aspects I needed to keep central when re-creating my practice and helped when I became stuck thinking in words.

\footnotetext{
${ }^{75}$ I do not believe that it is bad to need therapy and do not wish to create stigma around it. Therapy can assist in reflexivity and there is value in seeking help. This comment was made to highlight that we need to recognise that social/cultural discourses around disability can have negative impacts on disabled people that create a need for therapy. By exploring alternative discourses, I did not have to personally carry the baggage of stigma or prejudices and therefore did not seek therapy to the extent that I think I would have, had I not became involved in disability politics.
} 
- Creative writing helped me to develop new words and ways to describe my experience and to understand feelings associated with different discursive positions.

- Revisiting previous compostions provided further exploration and representation of my changing subjectivity. Creating music was a coconstructive force.

- Discovering photographs I had previously taken, which reminded me of particular thoughts/themes of the research, created a space between words to sit with particular problems/thoughts as well as providing a representational devise. I added most of the photographs after the first draft, providing moments of reflection during the editing process.

This movement from linguistic to other artistic representations helped me to see if my thoughts were still relevant in different forms or if they needed tweaking. Furthermore, I only found the words to describe some aspects after spending time in abstract modes of thought. Hoskyns (2017) describes the role of improvisation in fostering alternative ways of thinking in research.

My creativity was extended beyond the typical ways in which I am usually creative during the research process. Historically, music was my mode for creativity. However, after the onset of illness I developed a new interest in writing and poetry, which peaked during my second year of research as it was given more purpose. I also explored visual imagery (photographs). Exploring new creative mediums assisted the reflexive process and ethical task of creating the self as a work of art.

Additionally, the use of art/creativity was needed to address issues of representation. Language and representation devoid of emotion and imagination could not support this body of mine and the experiences I had. Accordingly, when representing MT, clients' poems, creative writings or art have been included in reports and as a source of data in this research. The increased inclusion of arts-based processes helped to decrease the gulf that can separate research, practice and experience. 


\subsection{Fostering awareness of the contextual space of therapy}

The methodology increased my awareness of the contextual space of therapy, thus assisting with reflexivity and the ethical care of the self. At the beginning of my $\mathrm{PhD}$, a lecturer said to me, "a fish doesn't know the water it swims in". It was my role as researcher to get to know that water. Because the research method demanded a constant dialoguing of both personal and sociocultural domains, it facilitated greater awareness of the contextual space of my MT practice. The methodology helped me to uncover the hidden curriculum which acculturates music therapists in NZ, such as the medical model that underpinned the standards of practice (chapter 5). With the increased awareness of the contextual shaping of disability, engaging in disability politics became a necessary part of my work: writing submissions for the new disability strategy (2016), proposing changes for the standards of practice in NZ (appendix 3). Consequently, PAMT's agenda, to create an environment and experience that is less disabling alludes to a process of people in context.

\subsection{Fostering support from the literature}

Whilst doing my research, I found books, articles, and blogs written by people who had had parallel experiences of illness/disability. I found this to be part of the care of the self as it always felt like a form of support to read these. Havi Carel's (2013) description of undergoing experimental treatment, the embodiment of forgetting how to run, the letting go of ideas such as having children (then later managing to go through a pregnancy) and her encounters with doctors who lacked empathy, felt hauntingly like she had written the story of my late 20 's and early 30 's. Such encounters through written words remind that despite differing diagnoses, we are not alone. I was never isolated in the experiences I was writing about.

\subsection{Music therapy self-care literature and summary}

Knowing oneself is the first step in taking care of oneself (Foucault, 1997b; Packer, 2011) and this first step is well acknowledged in MT literature. Importance has been placed on self-reflective and reflexive practice for students and registered therapists (Barry \& O'Callaghan, 2008; Rigney, 2011). Self-care for therapists has been written about (Trondalen, 2016) including creative arts-based self-care practices (Kaiser, 2017). Within the confines of this research, no literature was found on the use of 
Foucault's critical ontology of ourselves to support ethical care of the self for music therapists, and in this way, I hope this research offers a novel way to extend the current tool-kit.

In summary, the research methodology enhanced the ethical care of the self through the aspects noted above. This was important because MT cannot separate itself from culture (Pavlicevic \& Ansdell, 2004; Stige, 2005), therapists cannot separate themselves from the work (Aldridge, 1993) and there is an iterative relationship between practice and research in which they both influence each other (Hoskyns, 2013). I do, however, acknowledge that although the methodology may be beneficial in this way, there were moments during the process where disrupting what I had previously known became really unsettling and uncomfortable. Whilst this unsettling was a necessary part of the process, it perhaps only becomes beneficial if one can act and find other positions in one's practice. Otherwise it may result in feeling overwhelmed and stuck or cause the discontinuation of practice. For me, the creative process is what moved me through unsettled times, as I was aware of the potential to create something different. It is then not surprising to me that Foucault insisted that part of the ethical care of the self was to create the self as a work of art.

Feet stuck: body movers buried from sight in the guggliwugs of sand.

Body frozen: cold tides move in and out.

Hands create: sand art between waves, changing landscapes.

Mind eases: legs no longer willed to be free.

What did creativity mean to me? she asks:

It meant that beauty and meaning were found in stuck places;

Wanting to remain exploring and breathing, instead of waiting to drown. 


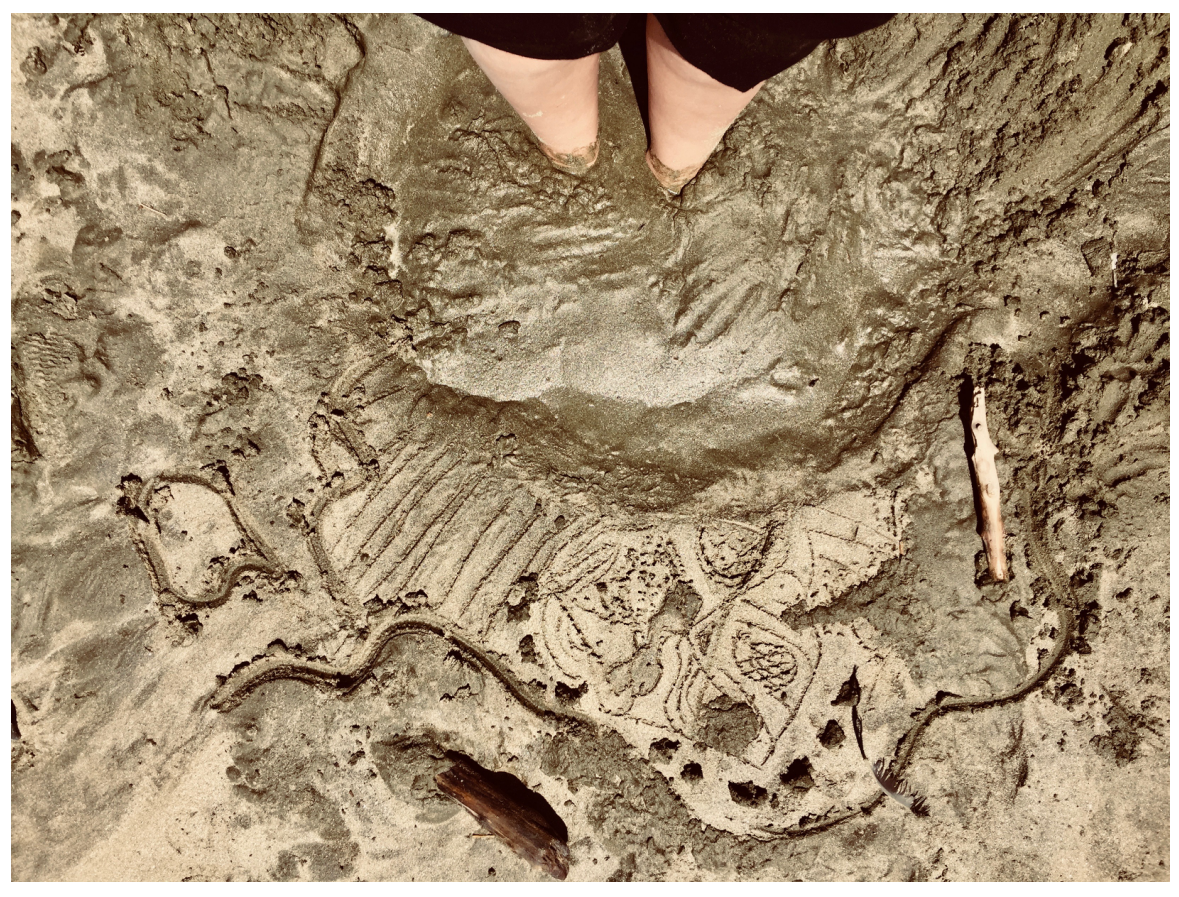

[Photograph of feet stuck in sand with some surrounding drawings/markings] 


\section{Chapter 11}

\section{Final thoughts: Reflections, Recommendations and Conclusion}

"Place of self-care": Photograph of Teal Bay, family holiday destination

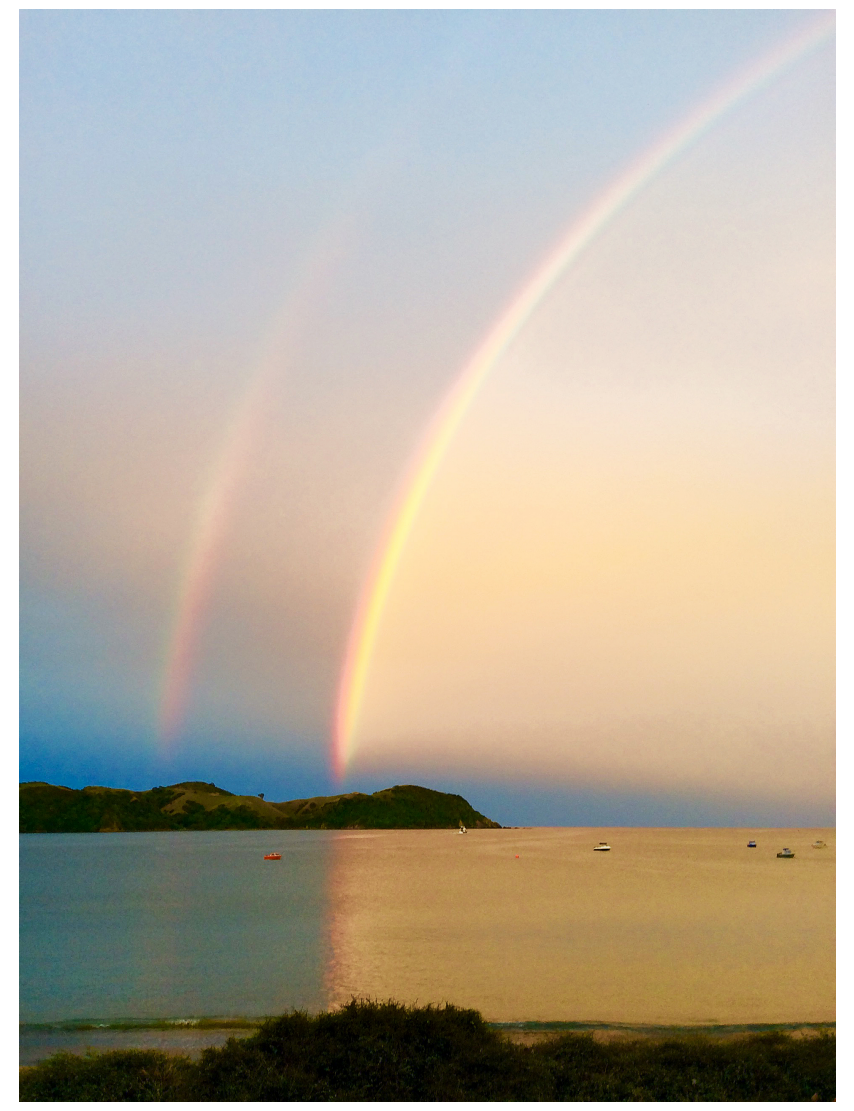

[Photograph of the sea, headland and two rainbows] 
This chapter will discuss my reflections on the research process, recommendations and finish with some conclusions.

\section{Reflections on the research process: Challenges and limitations (or are they?)}

I will discuss the following challenges and/or limitations:

- Balance

- Subjectivity and intersectional identity

- Relevance

- Challenges for the reader

- The ethics of personal disclosure

- Limitations of PAMT

\subsection{Balance}

One challenge during the research process related to balance. Trying to ensure that the research was rigorous, theoretical, analytical and emotional, evocative, creative and inclusive of personal and social phenomena, was a lot to negotiate. I tussled with questions of, am I being artful enough or too artful? Is there too much personal narrative or not enough? Is there enough sociocultural analysis or does it overpower and disrupt my narrative? Am I being too evocative or not evocative enough? Maintaining balance felt like an impossible challenge. Autoethnography is often criticised for either being too artful and not scientific, or too scientific and not sufficiently artful (Ellis et al., 2011). However, thinking in terms of balance made me fall into the dichotomies present in the above questions. These criticisms and balance struggles, position art and science at odds with each other. Autoethnography seeks to disrupt this binary. I had to let go of the idea of balance to escape dichotomous thinking. When it came to writing and representing my findings, I tried to be guided by what I needed to do to effectively answer the research questions and adequately represent my experience. 


\subsection{Subjectivity and intersectional identity}

I acknowledge that the problematisations, "truths", power effects, and ethical concerns raised were limited by my own intersectional identities and subjectivity. I am a white, heterosexual, cisgendered, sometimes disabled, sometimes ill, woman, born into a middle-class family and now of low-income. A music therapist with different intersectional identities with disability may have found different problematisations than those I have presented. Alongside disability, being female, and a low income earner, I also have the unearned privileges (McIntosh, 1988) associated with being heterosexual, cisgendered, and a NZ European. Importantly, my subjectivity was fluid and changed throughout the research, even within identity categories.

Differed aspects of my identity and forms of oppression interacted with each other in interesting ways. I had not been so confronted with the discrimination of being female until I experienced chronic illness/disability. Garland-Thomson (1997) describes the many parallels between the social meanings attributed to female bodies and those given to the disabled. Both are cast as deviant and inferior, excluded from full participation in economic and public life, and oppositional norms that are considered to possess physical superiority define them. Just like many others have written and described before me (Roger, 2014), being female impeded my care at times, which may in part be due to the discursive production of femaleness being equated with disability (Garland-Thomson, 1997). My reduced capacity was perhaps initially seen as part of being female, or as one doctor put it, "usually we see this kind of thing in busy mums, it becomes too much for their bodies" 76 .

Due to intersectional differences and different standpoints it perhaps goes without saying that while this research includes some of my experiences with illness/disability I am not the voice of the disabled. I am one voice in amongst many different voices. I recognise that other people in the music therapy (MT) profession may have personal experience with disability or chronic illness and that their experiences may differ to the ones I have discussed. However, they are no less valid or important and I hope

\footnotetext{
${ }^{76}$ I wasn't a mother at this particular point in time, so he seemed perplexed as to why I had developed the symptoms I was presenting with.
} 
this conversation extends beyond this study. We need to hear from a wide range of voices with different intersectionalities.

\subsection{Relevance}

My research is time bound and context specific, I am a historically located subject. The outcomes were found between 2014-2019, working in primary and secondary schools in New Zealand (NZ). My findings may not be relevant at a later time or in a different context. Whilst this lack of transferability through time and across place may seem limiting to the application of my findings, it may be a cause for celebration. If the findings are not relevant, it means that ableism is not an issue that warrants particular focus in MT in order to destabilise it.

Already, while I was doing my research, practices and ideas are changing. The standards of practice are currently being changed in NZ, accessibility needs for disabled therapists are being considered at professional events, and nearby in Australia disabled people are being employed as experts in research projects (McFerran, 2018). Whilst the relevance of ableism findings may change, my mode of inquiry and analysis tools may be useful for other research projects. It was developed as an adaptable and flexible approach that can be translated and further developed for each particular inquiry for the analysis of specific problems in specific contexts (Foucault, 1984b; Yates \& Hiles, 2010).

\subsection{Challenges for the reader and researcher}

This research may be challenging for some to read as it is messy at times with contradictions, confronting and confusing ideas, and complexity. It is part of a "less comfortable social science full of stuck places and difficult philosophical issues of truth" (Lather, 2006, p. 35). It demands a lot from its reader as it delves into art representations that perhaps take more time to process. Autoethnographers challenge the notion that research should lead to clear, tidy conclusion. Instead they present phenomena as they are: messy, complicated and confusing (McGraw Hunt, 2016). Whilst necessary, presenting the messy and complicated experience of illness was a challenging aspect of the research process. I wanted to put things in boxes, but then realised those boxes didn't fit. As a result, there is necessary contradiction and 
perhaps my confusion is present at times too, due to living in multiplicities. These contradictions, however, formed the productive possibilities of agonism.

It was a helpful reminder that the intelligible is not synonymous with limit as what exists does not fill all possibilities (Foucault, 1997a, Lather, 2006). To remain inside intelligibility produces a "constitutive outside, the unspeakable, the unviable, the nonnarrativizable" (Butler, 1993, p. 188). To remain in one discourse or to adhere to its rules of formation would continue to create an outside (Lather, 2006). At times the narratives have subverted the coherence of the discourses, such as in examples where doctors practicing within the medical model adopted mutuality and resisted paternalistic power relations: adapting the formation of the medical model.

I recognise that one may feel discomfort in reading this research, as I too experienced disruptions and disturbance as my awareness of ableism grew. To add to this discomfort, the research does not find all of the answers to the problematisations raised. As Shakespeare (2012) pointed out, as a researcher it has been my role to ask the difficult questions and to present what I find even when it is inconvenient, challenging and when there are no easy answers.

\subsection{Personal disclosure}

Whilst self-examination can be difficult to endure, for me the element of public disclosure has been the most confronting. I was aware throughout my research that writing my personal experiences in this public forum of the thesis could not guarantee me a safe place in which to speak (Kamler, 2001). I live in a society that continues to accuse disabled people of being overly sensitive when pointing out problematic attitudes/actions and often their experiences are minimised.

Furthermore, as with any long-term health condition, access to medical care is still needed and I am mindful of the consequences of being labelled as a difficult patient. I have necessarily needed to navigate the system in a manner that doesn't offend the doctors who are the gatekeepers to the most effective treatments. That is, I need to constantly negotiate effects of power to access the best care. Indeed, I have contemplated the potential fallout I may experience should any treating doctor become aware of this research. While being investigated for new spinal cord symptoms towards the end of my study, I became painfully aware that I could not 
ethically print this thesis under the full name I go by: I felt strangled by what I could write. I explored this in the journal entry below.

My name is voiceless

The first neurologist I saw was the first to steal it.

"I am taking this patient" he said to the nurse

Wheeled me prisoner without no name.

My slow responses made him yell.

"I don't have time for this" he said

If not him, then who would care for a body that slowed time.

My name cried, there were no words to be found,

An unknown body dialect that I had yet to learn.

“Are you just depressed?" the neurologist huffed.

My name is female, the descriptions of my body not to be believed.

So "stand up' he says, "or can you not do that either".

He shushes my husband for answering.

My name, speechless, stood with four hands pressing to find a pulse.

"Have you got it?" he asks the other man with no name.

“I'm struggling, it's so weak, lie her down", he pleads.

My name need not know what the four hands found.

"Tell Doctor Murphy, he will look after her now" he said.

Taken back to the ward with the large patient file weighing down on my knees.

From that examination on, my name has been strangled to not speak of the violence.

I was to remain the "good patient", to gain the rewards.

Above and beyond they will go if they like you. 
My name closed its mouth, treatments it got.

While the difficult ones lay waiting at home.

There is nothing more they can do for them.

My name listened to the urologist direct his explanations towards my husband.

His averted gaze communicating that my husband needed to understand this more than I did.

He could only offer pills used for older men with prostate problems.

My name was rejected by some.

If they didn't know what to do, they discharged.

Discharged of their call to care or to consider what else might be.

My name has a body that many have much to say about.

I sat as the case for 12 neurologists to audit.

Only speaking when spoken to.

My name listened to its patient history.

"complicated" and "interesting" the key descriptors once more

Humiliated by being reduced to a list of diagnoses and grey areas they still could not understand.

My name was there to answer questions or to show them the "spastic gait".

Not one of the 12 asked if we had questions or anything further to add.

Excused, I was told to wait out there while they discussed.

My name has no authority on this body.

The absent special guest. My chair is emptied.

The one always in need of this system to heal, And the one wounded by it. 
My name is voiceless

I can no longer write under it

Upon this name will always remain louder voices than my own

I have drowned beneath them.

I need to find a new name from which to speak.

Not submitting under the full name I go by helped to free my writing. Although, there were still some things I left out, as they did not sit well with me to share, and some things left in still feel on the edges of what is comfortable.

None-the-less, this autoethnography was not about self-confession of authentic feelings or truth, but about intertextual positioning and care of the self. I have therefore tried to encapsulate the key issues and discourses that were illuminated by my narrative positions with the material included. The sharing of these narratives was not about helping others to see the world through someone else's eyes, as I now appreciate that one can never stand in someone else's shoes. Rather they were used to highlight that the ethical relation starts with the willingness to be open to everyone's unique, embodied subjectivity.

I ask that the reader understands and relocates my personal narratives as the province, not of simply a private individual, but of social, cultural and political spaces (Kamler, 2001). I ask this perhaps because it is difficult to navigate the fact that ultimately the people reading judge for themselves what experiences count as relevant and what kind of reading is correct (Kamler, 2001). By seeing the personal as political and sociocultural, all experience then counts and there are many different readings possible that I look forward to hearing and engaging with in discussions to come.

\subsection{Limitations of PAMT}

As already mentioned, PAMT isn't a fixed orientation and therefore it is hard to precisely define. To generalise my use of PAMT would be to present MT and ableism in a narrow way. This limited ability to define it allows for new or different forms of ableism that might emerge in another context to be considered. Not allowing for flexibility may enforce a particular way of being. The understandings of PAMT 
presented in this thesis are constrained by the timeframe of my PhD. For example, it was not possible to explore further examples of its use or application in a different area of practice, which may have led to further adaptions, extensions and definitions.

There are limitations in the PAMT aim of reducing barriers and ethos of mutuality and collaboration. A utopia that is barrier-free is incredibly difficult to realise and there may be some limitations to the level of collaboration, which needs to be carefully negotiated. Sometimes clients may not want to collaborate and prefer the therapist to be in an expert role. For example, personally there were times when I would get exhausted or overwhelmed by having to manage symptoms and work with health professionals (sharing information between specialists, suggesting ideas, organising and arranging suggested tests, emailing them progress). I would have preferred to have had an expert with all the answers that did everything. Additionally, working collaboratively together does not always mean working effectively.

As PAMT draws on posthumanism, it is helpful to consider critiques of posthumansim. One critique is the irony of its use of human methods of discourse and scholarship to make its critical case (Ansdell \& Stige, 2018; Peterson, 2018). However, this just demonstrates the stronghold of humanism and it is important to acknowledge that posthumanism isn't a destination but a process of becoming. Posthumanism has also been critiqued for not articulating a comprehensive ethic for how individuals should respond to technology (Benko, 2005). Technology has led to a new group of marginalised people (Wolbring, 2009), such as, human enhancement technology may be enabling for a few but the cost may be prohibitive for many. Technology has also historically been associated with normalisation (Goodley, 2010; Reeve, 2012).

Trying to utilise a PAMT orientation in some situations might be challenging simply because of the contexts in which we work or the professional mandates that hold. Funding priorities are still often given to those with the most visible disabilities and we cannot work without funding. I still have to participate in specialist service standards reviews at school that measure what success is in particular ways. I have to confirm that I will comply with the MT code of ethics and meet the service standards to renew my practicing certificate, even though some of it conflicts with the ethics presented in PAMT. I write ORRS applications in the style of the deficit model of 
disability, as this is the only way to help students get the funding they need to access MT and other support. Simply put, I work in the muddied water where I temporarily shelve the position of PAMT when needed so I can then continue with my work as a post-ableist music therapist. Therefore, it is not a position that is always possible to maintain. It is one to keep returning to, one to keep pushing the boundaries where possible, one that thinks outside of what is possible, and one that with quiet (or not so quiet) consistent persistence intends to reduce some of the ableism that is epidemic in society.

Furthermore, not all challenging and difficult aspects of disability stem from ableism. As indicated previously, sometimes no amount of social justice or medicine brings into relief that which disables me. Although this may seem like a short fall of PAMT, the challenges of illness/disability are significantly heightened when ableism is at play. It is still relevant to consider, even when addressing it can't always directly relieve and assist. Despite the limitations and potential challenges of PAMT, accounted for or unaccounted for here, this framework is another step towards a more robust theorisation of ableism in MT.

\section{Recommendations}

\subsection{Increase attention to processes of ableism and ways to address it in practice}

After presenting some preliminary findings of my doctoral research (Shaw, 2016), a colleague asked me, "but what do we do about this"? I wanted to have all the answers, but I didn't then, and I still do not hold a full deck of cards. We can only play strategically with what we have whilst trying to change the rules. We each have our own set of cards and therefore, I cannot tell her exactly how to play her hand. However, from this research the general recommendation would be to increase attention to processes of ableism and create ways to address it. In this section, I will discuss how this may be achieved through:

- Listening to and including disabled people, communities, and cultures

- Extending and further developing the lens of PAMT

- Using the tools of poststructural arts-based autoethnography 
The strategies above may support one in becoming an ally. Oswanski \& Donnenwerth (2017) explain that to become an ally one must examine issues of oppression and disrupt disadvantage and marginalisation. The authors note that as music therapists we are not automatically allies, we need to undertake considerable education, selfawareness and direct-action practice. The strategies discussed below support such an undertaking and promote ally behaviours. When applying such strategies, like Oswanski \& Donnenwerth, (2017) suggest, humility supports best practice.

Firstly, I recommend listening to, including and engaging with disabled voices, communities and cultures on multiple levels. Include disabled people in the strategic planning of professional bodies. Invite disabled people and communities to conferences and as guest speakers ${ }^{77}$. Become familiar and engage with disability studies. Have awareness of crip music movements, crip theory, the neurodiversity movement, and disability pride. In practice, honour partnership with disabled people. Provide people with platforms to contribute to reports, assessments and research to foster mutuality ${ }^{78}$. In doing so, a space is created for silenced/unheard voices to inform practice. Ultimately, disabled people should have a stake in controlling the discourses that so largely control their lives (Lubet, 2011).

Interestingly, Gross's (2018) summary of the practical take home messages derived from the Voices special issue on MT and disability studies (2014), confirms the importance of listening to and inclusion of disabled voices. The take home messages were: we need to be aware of the power effects of hierarchies (LaCom \& Reed 2014), do not rely on observational measures (Bassler, 2014), allow clients to participate in their own goals (Rickson, 2014), and collaborate with disability scholars and activists (Cameron, 2014; Metell, 2014). All are addressed through increased listening and inclusion of those experiencing ableism.

Secondly, I recommend that PAMT is continually extended through creativity. Given that ableism was found to exist across MT discourses, PAMT is a theory of ideas that can be applied to any practice orientation to help guide one in working out what to do.

\footnotetext{
${ }^{77}$ Whilst not common practice, this is beginning to occur in NZ (Rickson, 2014a). For example, at the music therapy conference in 2017, participants from the Wellington Neurological Choir came to present their choirs work.

${ }^{78}$ This should not be confined to verbal contributions, but to include the unique ways that people may communicate.
} 
PAMT brings disabled identity politics into consciousness and recognises agonistic spaces as enabling sites for working through the stuck places of present practice. Within posthumanism, new practices and possible futures are actualised and generated affirmatively and creatively by mobilising resources and untapped visions through daily practices of interconnection with others (Braidotti, 2013). Therefore, further creative development is important.

Thirdly, I suggest using the tools presented in arts-based autoethnography and poststructural analysis, not just as a way of researching, but for the personalised use of processing MT work. Such tools included, looking for problematisations, moments of governmentality, creative processes, positioning self-narratives. For example, to help MT students uncover techniques of the self they could explore a problematisation in their work and locate moments of governmentality, or therapists could write a piece of poetry to explore in supervision for ethical care.

\subsection{Research directions}

It may be useful if directions of research focus towards exposing the processes through which the normate is created, agreed and maintained. Instead of examining disability from the perspective of the other, research projects could include examinations around what the study of disability tells us about the production, operation and maintenance of ableism. Including this reframing would foster questions like, what does the study of Autistic people's music making tell us about normative music making ideals and neurotypical music making in MT?

\subsection{Recommendations for therapists who experience illness/disability, professional bodies, and NZ context}

For therapists who experience illness/disability, the tools from the research methodology were helpful in navigating the position and issues I encountered. The tools supported the integration of professional and personal narratives to work with, not against, each other in finding less restrictive spaces for clients and myself. Overall, there was an increased 'care of the self'. Apart from the tools this research offers, I do not have so much as further recommendations for managing the ableism one may encounter in their practice and studies, but a reaching out. Like some have already done so before me, I am waving across the pages. Feel free to wave back. 
Professional bodies and MT communities may wish to reflect on how they include therapists with disabilities and determine whether there are any exclusionary or discriminatory practices. Indeed, more research needs to be done in this area. How many therapist's experience illness/disability? Is there a reason that not more of us have written about our experiences? What continues to be hard to say? To assist with increasing professional inclusion, I suggest that we continue to broaden our collective understandings of disability as this may reduce some of the barriers that disabled therapists face. Such visibility and inclusion is important as until the MT field is representative of the diverse range of people it serves, ableism can only be explored academically. As a participant in a study exploring queer MT suggests, "our field is a mess because we do not reflect our clients in any way...We're not drawing from people that could bring diversity to our field" (Boggan et al., 2017, p. 386).

I recommend that we continue to diversify practice in order to increase available positions and extend what is currently available for disabled people in NZ. For example, developing a disabled people's choir that supports positive identities and welcomes disabled allies might be one such alternative. The choir might sing repertoire that rejects, challenges or disrupts normalised stereotypes of disabled people. It may provide an alternative to the neurological choirs in NZ currently, which group people according to set diagnoses, assuming they have similar issues due to their impairment. I feel uncomfortable knowing that I would not find a place in such a choir (despite having a neurological impairment) and alternative options might be helpful for others too.

\subsection{Self care}

It is probably at the edges of research recommendations, but we so often recommend all the work that needs to be done that I also suggest going to the beach or anywhere else to find some beauty and stillness. In these moments of sanded toes and windswept hair, it is possible to appreciate and be reminded that our worth has nothing to do with ability. In these moments, there is a self-care between the confrontations of all the work that still needs to be done, the disappointments, and injustices. PAMT will not always go smoothly. Therefore, I recommend that there is a care of the self and grounding in the quest to social justice. 
Perhaps this recommendation is actually for myself: the end of this thesis feels like a finish line that keeps moving away the closer I get to it. I am puffing while my walk slows. The sea is calling with its lulling hush. The energy needed to see the effects of power, the courage to speak of it, to creatively rework oneself as an ethical work of art can only be rejuvenated by rest. The sustainability of social justice endeavours is critical as there is no finish line in poststructural theory. Social transformation is an ongoing process in the work that we do, in the contexts we do it, and in the language we use (it is discursive, local, and individual).

\section{Conclusion}

This research builds on the important work of critical and social-justice based music therapists. Whilst it raised critical dilemmas in my own practice, it also raised ethical issues and reflections for the field of MT to consider. There is a need to continue to extend and develop our collective understandings about what it means to be disabled and ill to encompass diversity and different ways of being. This research discovered alternative, subordinate understandings of illness/disability and explored the extension of these within practice. Indeed, it suggested a cripping of MT practice that could not simply be incorporated into existing frameworks.

The ethical issues and power effects raised in the analysis were understood under the umbrella of ableism. Uncovering the depth of the roles I played in ableism through the constructed "truths" in the prior practice studied, and the contextual shaping and encounters of ableism in education and the profession, was the most significant finding. Although many examples of ableism were found, admittedly I am aware that I am still oblivious to other forms and examples. The depth of ableism has not been a comfortable finding to share, but it has been necessary to not shy away from presenting the rawness and extent of it in order to avoid complicity. It demonstrated that while a therapist and the contexts she inhabits may not consciously profess to be against disabled people, there were invisible forms of ableism within the studied practice that were previously unknown. This calls for the need to address the undertheorisation of ableism in MT.

Whilst critiques of various approaches are made throughout this research, they were not based on negative grounds but as productive attempts to find and define 
alternative positions from which to view and address gaps in current practice. It is hoped that this research helps us as a profession not to evade ableism, increase awareness of forms of ableism, and acknowledge and accept that although we intend to do good, we may not be innocent in the oppression and discrimination of others. As ableism impacts on everyone, to not act supports not only the confining and restraining discrimination of disabled people but the detriment of others too. With an attention to social justice, the position of a therapist who experiences illness/disability becomes an easier one to manage/negotiate.

One aim was to bring into relief the fundamental ambivalence I felt as one who had previously shaped a professional identity based on specific meanings of disability and illness, and some of the internal conflicts that were amplified by my personal experiences. This was resolved (partially) by an increased ethical care of the self that was fostered through an arts-based autoethnographic critical ontology of the self which enacted upon ableism through the development of PAMT. PAMT extends the relational ethic beyond what was present in the prior practice by drawing on aspects of posthumanism (valuing interdependence), agonistic pluralism, and increasing the incorporation of disability studies and crip theory. Due to the ableist tendencies of humanism, posthumanism was used as a foundation in PAMT, which differs to current MT orientations. No MT theories/orientations were found within the confines of the research that claimed and embraced posthumanism as a foundation for practice. Whilst some therapists have offered critiques of humanism, there still appears to be a desire to "hang on" to this dominant discourse (in extended forms) in contemporary practice at the cost of developing increased pluralistic foundations and agonistic pluralism. Therefore, PAMT is offered as an alternative lens in the critical orientations' apparatus: a social justice practice that is not based on empowerment and humanism but instead on agonism and posthumanism.

The "truths" discovered in the practice studied and the particular forms of ableism found are not generalisable to all MT practice, nor were they meant to be. Instead, the investigation demonstrates methodological tools that may help to free oneself from certain understandings, subvert normalising dangers in discursive practices and cultivate new meanings and practices. Indeed, the critical ontology of ourselves is considered as an ongoing ethos, attitude, and philosophical way of life that critiques what we are and experiments with going beyond the limits imposed on us. 
Accordingly, I finish this thesis knowing that this conclusion is not a final statement of thought, as any finality would stagnate further developments of practice that may politicise disability and address ableism. For me, this conclusion is instead a starting point, not the end. And so, I begin... 


\section{References}

Aarø, L., \& Stige, B. (2012). Invitation to community music therapy. New York: Routledge.

Abrams, B. (2010). Evidence-based music therapy practice: An integral understanding. Journal of Music Therapy, 47(4), 351-379. http://doi.org/10.1093/jmt/47.4.351

Abrams, B. (2018). Understanding humanistic dimensions of music therapy: Editorial introduction. Music Therapy Perspectives, 36(1). http://doi.org/10.1093/mtp/miy019/5049771

Achieve. (2004). Kia orite achieving equity: New Zealand code of practice for an inclusive tertiary education environment for students with impairments. New Zealand: Tertiary Education Commission, Ministry of Education.

Aigen, K. (2005). Music-centered music therapy. Gilsum, NH: Barcelona Publishers.

Aigen, K. (2008). In defense of beauty: A role for the aesthetic in music therapy theory. Nordic Journal of Music Therapy, 17(1), 3-18. http://doi.org/10.1080/08098130809478191

Aigen, K. (2009). The importance of faith: A commentary on Bower's and Shoemark's article. Australian Journal of Music Therapy, 20, 76-77.

Aigen, K. (2014). The study of music therapy: Current issues and concepts. New York: Taylor and Frances.

Aigen, K. (2015). A critique of evidence-based practice in music therapy. Music Therapy Perspectives, 33, 12-24. http://doi.org/10.1093/mtp/miv013

Albrecht, G. (Ed.). (2006). Encyclopedia of disability. Thousand Oaks, CA: Sage Publications, Inc.

Aldridge, D. (1993). Music therapy research II: Research methods suitable for music therapy. The Arts in Psychotherapy, 20, 117-131.

Allan, J. (2006). Inclusion as ethical practice. In S. Tremain (Ed.), Foucault and the 
government of disability (pp. 281-297). USA: The University of Michigan Press.

Alvesson, M. (2002). Postmodernism and social research. Buckingham, UK: Open University Press.

Amir, D. (1996). Issues in qualitative research: A personal journey. In L. Mechtild, K. Aigen, \& J. Frommer (Eds.), Qualitative music therapy research: Beginning dialogues (pp. 213-224). Gilsum, NH: Barcelona Publishers.

Anderson, L. (2006). Analytic autoethnography. Journal of Contemporary Ethnography, 35(4), 373-395. http://doi.org/10.1177/0891241605280449

Annesley, L. (2014). The music therapist in school as outsider. British Journal of Music Therapy, 28(2), 36-43.

Ansdell, G. (1995). Music for life: Aspects of creative music therapy with adult clients. London, UK: Jessica Kingsley Publishers.

Ansdell, G. (2002). Community music therapy and the winds of change. Voices: $A$ World Forum for Music Therapy, 2(2). http://doi.org/http://dx.doi.org/10.15845/voices.v2i2.83

Ansdell, G. (2003). The stories we tell. Nordic Journal of Music Therapy, 12(2), 152159. http://doi.org/10.1080/08098130309478085

Ansdell, G. (2014). How music helps in music therapy and everyday life. Farnham Surry, England: Ashgate Publishing, Ltd.

Ansdell, G., \& Stige, B. (2018). Can music therapy still be humanist? Music Therapy Perspectives, 36(1). http://doi.org/10.1093/mtp/miy018

Aronson, J. K. (2000). Autopathography: The patient's tale. British Medical Journal, 321(7276), 1599-1602.

Ashmore, R., \& Banks, D. (2002). Self-disclosure in adult and mental health nursing students. British Journal of Nursing, 11(3), 172-177.

Atkinson, P. (2006). Rescuing autoethnography. Journal of Contemporary Ethnography, 35(4), 400-404. http://doi.org/10.1177/0891241606286980 
Audet, C. (2011). Client perspectives of therapist self-disclosure: Violating boundaries or removing barriers? Counselling Psychology Quarterly, 24(April), 85-100. http://doi.org/10.1080/09515070.2011.589602

Austin, D. (2002). The wounded healer. The voice of trauma: A wounded healer's perspective. In J. Sutton (Ed.), Music, music therapy and trauma. International perspectives (pp. 231-259). London, UK: Jessica Kingsley Publishers.

Austin, D., \& Forinash, M. (2005). Arts-based research. In B. Wheeler (Ed.), Music therapy research (2nd ed, pp. 458-471). Gilsum, NH: Barcelona Publishers.

Austin, D., Mahoney, J., Courter, A., Ryan, K., Starace, J., \& Defeo, N. (2016). Artists' reflections on preparing and performing the arts-based research study Grace Street. Music Therapy Perspectives, 34(1), 48-51. http://doi.org/10.1093/mtp/miw001

Averett, P., \& Soper, D. (2011). Sometimes I am afraid: An autoethnography of resistance and compliance. The Qualitative Report, 16(2), 358-376.

Axline, V. (1964). Dibs, in search of self. New York: The Random House Publishing Group.

Ayson, C. (2011). The use of music therapy to support the SCERTS model objectives for a three year old boy with autism spectrum disorder in New Zealand. The New Zealand Journal of Music Therapy, 9, 7-31.

Bailey, E. (2010). The sound of a wild snail eating. Melbourne, Australia: Text Publishing.

Bain, C., Grzanka, P., \& Crowe, B. (2016). Toward a queer music therapy: The implications of queer theory for radically inclusive music therapy. Arts in Psychotherapy, 50, 22-33. http://doi.org/10.1016/j.aip.2016.03.004

Baines, S. (2013). Music therapy as an anti-oppressive practice. Arts in Psychotherapy, 40(1), 1-5. http://doi.org/10.1016/j.aip.2012.09.003

Baines, S. (2014). Giving voice to service user choice: Music therapy as an antioppressive practice (unpublished doctoral dissertation). University of Limerick, Limerick, Ireland. 
Bakan, D. (2014). A song of songs: A/r/tography, autoethnography, and songwriting as music education research (doctoral dissertation). The University of British Columbia, Vancouver, Canada.

Bakan, M. (2014). The musicality of stimming: Promoting neurodiversity in the ethnomusicology of autism. MUSICultures, 41(2), 133-161. Retrieved from http://search.ebscohost.com/login.aspx?direct=true\&db=a9h\&AN=103698534\& site $=$ ehost-live

Bakan, M. (2015). Toward an ethnographic model of disability in the ethnomusicology of autism. In B. Howe, S. Jensen-Moulton, N. Lerner, \& J. Straus (Eds.), The Oxford handbook of music and disability studies (pp. 15-36). Oxford UK: Oxford University Press.

Bakan, M. (2016). Music, autism, and disability aesthetics. Journal of the American Musicological Society, 69(2), 548-553.

Baker, F. (2017). Re-authoring the self: Therapeutic songwriting in identity work. In R. MacDonald, D. Hargreaves, \& D. Miell (Eds.), Handbook of musical identities. Oxford UK: Oxford Scholarship Online. http://doi.org/10.1093/acprof

Baker, F., \& Krout, R. (2012). Turning experience into learning: Educational contributions of collaborative peer songwriting during music therapy training. International Journal of Music Education, 30(2), 133-147.

Barker, C., Pistrang, N., \& Elliott, R. (2002). Research methods in clinical psychology: An introduction for students and practitioners. (2nd ed.). West Sussex, England: John Wiley \& Sons, LTD.

Barry, P., \& O’Callaghan, C. (2008). Reflexive journal writing: A tool for music therapy student clinical practice development. Nordic Journal of Music Therapy, 17(1), 55-66. http://doi.org/10.1080/08098130809478196

Bassler, S. (2014). "But you don't look sick": Dismodernism, disability studies and music therapy on invisible illness and the unstable body. Voices: A World Forum for Music Therapy, 14(3).

http://doi.org/http://dx.doi.org/10.15845/voices.v14i3.802 
Baxter, H., Berghofer, J., MacEwan, L., Nelson, J., Peters, K., \& Roberts, P. (2007). The individualized music therapy assessment profile. London, UK: Jessica Kingsley Publishers.

Behar, R. (1993). Introduction: Women writing culture: Another telling of the story of American anthropology. Critique of Anthropology, 13(4), 307-325. http://doi.org/10.1177/0308275X9301300401

Benko, S. (2005). Ethics, technology, and posthuman communities. Essays in Philosophy, 6(1), 1-17.

Berger, R. (2007). Choosing the "right" space to work in: Reflections prior to a nature therapy session. Australian Journal of Outdoor Education, 11(1), 41-44.

Bergmann, T., Sappok, T., Diefenbacher, A., \& Dziobek, I. (2016). Music in diagnostics: Using musical interactional settings for diagnosing autism in adults with intellectual developmental disabilities. Nordic Journal of Music Therapy, 25(4), 319-351. http://doi.org/10.1080/08098131.2015.1039567

Bevan, J. (2013). Disabled occupational therapists - asset, liability...or "watering down" the profession? Disability \& Society, 29(4), 583-596. http://doi.org/10.1080/09687599.2013.831747

Bhabha, H. (1994). The location of culture. London \& New York: Routledge.

Birk, L. B. (2013). Erasure of the credible subject: An autoethnographic account of chronic pain. Cultural Studies $\leftrightarrow$ Critical Methodologies, 13(5), 390-399. http://doi.org/10.1177/1532708613495799

Bodner, E., \& Polansky, E. A. (2016). The attitudes of music therapy students and professionals regarding the emotional valence of improvisations in music therapy. Nordic Journal of Music Therapy, 25(3), 273-295. http://doi.org/10.1080/08098131.2015.1067248

Bogart, K. (2017, October). Disability pride: Why disability is not a bad thing. EP Magazine, 32-34.

Bogart, K., Lund, E., \& Rottenstein, A. (2017). Disability pride protects self-esteem through the rejection-identification model. Rehabilitation Psychology, 
July(Advance online publication). http://doi.org/10.1037/rep0000166

Boggan, C., Grzanka, P., \& Bain, C. (2017). Perspectives on queer music therapy: A qualitative analysis of music therapists' reactions to radically inclusive practice. Journal of Music Therapy, 54(4), 375-404. http://doi.org/10.1093/jmt/thx016

Bolton, G., \& Delderfield, R. (2018). Reflective practice, writing and professional development (5th ed.). Washington DC: Sage Publications, Inc. http://doi.org/10.1080/03069885.2011.611410

Boylorn, R., \& Orbe, M. (2014a). Creating pathways to authentic selves. In R. Boylorn \& M. Orbe (Eds.), Critical autoethnography: Intersecting cultural identities in everyday life (pp. 189-192). Walnut Creek, CA: Left Coast Press, Inc.

Boylorn, R., \& Orbe, M. (2014b). Introduction. In R. Boylorn \& M. Orbe (Eds.), Critical autoethnography: Intersecting cultural identities in everyday life (pp. 13-26). Walnut Creek, CA: Left Coast Press, Inc.

Bradt, J. (2017). Threats to legitimacy? Nordic Journal of Music Therapy, 26(4), 291292. http://doi.org/10.1080/08098131.2017.1328033

Braidotti, R. (2013). The posthuman. London, UK: Polity.

Brinks, D. (2011). 4th of July demon moped. In J. Bartlett, S. Black, \& M. Northen (Eds.), Beauty is a verb: The new poetry of disability (p. 363). United States of America: Cinco Puntos Press.

Brooks, C. (2011). Social performance and secret ritual: Battling against obsessivecompulsive disorder. Qualitative Health Research, 21(2), 249-261. http://doi.org/10.1177/1049732310381387

Bruscia, K. (1987). Improvisation models of music therapy. Illinois: Charles C Thomas.

Bruscia, K. (1996). Daedalus and the labyrinth: A mythical research fantasy. In Langenberg, Aigen, \& Frommer (Eds.), Qualitative music therapy research: Beginning dialogues (pp. 205-212). Gilsum, NH: Barcelona Publishers. 
Bruscia, K. (1998). Defining music therapy. Gilsum, NH: Barcelona Publishers.

Bruscia, K. (2014). Defining music therapy (3rd ed.). Gilsum, NH: Barcelona Publishers.

Bunt, L. (2004). Mary Priestley interviewed by Leslie Bunt. Voices: A World Forum for Music Therapy, 4(2). http://doi.org/http://dx.doi.org/10.15845/voices.v4i2.180

Bunt, L., \& Hoskyns, S. (2002). The handbook of music therapy. Sussex, UK: Brunner-Routledge.

Butler, J. (1993). Bodies that matter: On the discursive limits of "sex." New York: Routledge. http://doi.org/10.1177/0306312706056409

Bybee, M. (2017). Music therapists and work: Experiences of occupational oppression in the profession of music therapy. Truman State University.

Cameron, C. (2009). Tragic but brave or just crips with chips? Songs and their lyrics in the disability arts movement in Britain. Popular Music, 28(3), 381-396. http://doi.org/10.1017/S0261143009990122

Cameron, C. (2014). Does disability studies have anything to say to music therapy? And would music therapy listen if it did? Voices: A World Forum for Music Therapy, 14(3). http://doi.org/http://dx.doi.org/10.15845/voices.v14i3.794

Campbell, F. K. (2006). Legislating disability: Negative ontologies of the government of legal identities. In S. Tremain (Ed.), Foucault and the government of disability (pp. 108-132). USA: The University of Michigan Press.

Campbell, F. K. (2008a). Exploring internalized ableism using critical race theory. Disability and Society, 23(2), 151-162. http://doi.org/10.1080/09687590701841190

Campbell, F. K. (2008b). Refusing able(ness): A preliminary conversation about ableism. Media and Culture Journal, 11(3), 1-14. Retrieved from http://journal.media-culture.org.au/index.php/mcjournal/article/viewArticle/46

Campbell, F. K. (2009). Contours of ableism: The production of disability and 
abledness. New York: Palgrave MacMillan.

http://doi.org/10.1080/10304311003797498

Campbell, F. K. (2014). Ableism as transformative practice. In C. Cocker \& T. Hafford-Letchfield (Eds.), Rethinking anti-oppressive theories for social work practice (pp. 78-92). London, UK: Palgrave MacMillan.

Carel, H. (2013). Illness. Durham, UK: Acumen Publishing Limited.

Carlson, L. (2013). Musical becoming: Intellectual disability and the transformative power of music. In M. Wappett \& K. Arndt (Eds.), Foundations of disability studies (pp. 83-104). New York: Palgrave MacMillan.

Carmody, B. (2018, March 29). "You look like a burns victim": Aussie radio host criticised for "deeply uncomfortable" interview with disability activist. Stuff. New Zealand. Retrieved from https://www.stuff.co.nz/entertainment/tvradio/102708766/aussie-radio-host-criticised-for-deeply-uncomfortableinterview-with-disability-activist

Carpente, J. (2013). IMCAP-ND: The individual music-centered assessment profile for neurodevelopmental disorders. North Baldwin, NY: Regina Publishers.

Carr, A. (1998). Michael White's narrative therapy. Contemporary Family Therapy, 20(4), 485-503. http://doi.org/10.1023/A:1021680116584

CCS Disability Action. (2013, August 13). Disabled people discriminated against in employment. Scoop: Independent News, p. 2.

Chacala, A., McCormack, C., Collins, B., \& Beagan, B. (2013). "My view that disability is okay sometimes clashes": Experiences of disabled occupational therapists. Scandinavian Journal of Occupational Therapy, 21(2), 107-115.

Challis, B. (2009). Technology, accessibility and creativity in popular music education. Popular Music, 28(3), 425-431. http://doi.org/10.1017/S0261143009990158

Chambers, S. (2001). Language and politics: Agonistic discourse in "The West Wing." Ctheory, a098a. Retrieved from http://www.ctheory.net/articles.aspx?id=317 
Chang, H. (2008). Autoethnography as method. Walnut Creek: Left Coast Press.

Chang, H., Ngunijiri, F., \& Hernandez, K. (2013). Collaborative autoethnography. Walnut Creek: Left Coast Press.

Charon, R. (2001). Narrative Medicine: A model for empathy, reflection, profession, and trust. The Journal of the American Medical Association, 286(15), 18971902.

Chatham-Carpenter, A. (2010). " Do thyself no harm ": Protecting ourselves as autoethnographers. Journal of Research Practice, 6(1), 1-13.

Churchill, V., \& McFerran, K. (2014). Developing a music therapy assessment tool specific to persons with severe to profound multiple disabilities. The New Zealand Journal of Music TherapyZ Journal of Music Therapy, 12, 8-33.

Churchill, W. (2015). Deaf and hard-of-hearing musicians: Crafting a narrative strategy. Research Studies in Music Education, 37(1), 21-36. http://doi.org/10.1177/1321103X15589777

Claiborne, L., Cornforth, S., Gibson, A., \& Smith, A. (2011). Supporting students with impairments in higher education: Social inclusion or cold comfort? International Journal of Inclusive Education, 15(5), 513-527. http://doi.org/10.1080/13603110903131747

Cloyes, K. (2002). Agonizing care: Care ethics, agonistic feminism and a political theory of care. Nursing Inquiry, 9(3), 203-214.

Coleman-Brown, L. (2013). Stigma: An enigma demystified. In L. Davis (Ed.), The disability studies reader (4th ed., pp. 147-160). New York: Routledge: Taylor and Francis Group.

Collins, P. (1990). Black feminist thought: Knowledge, consciousness, and the politics of empowerment. Boston, MA: Unwin Hyman.

Cornforth, S. (2006). A discursive approach to the registration debate: Working with texts; Thinking ethics. New Zealand Journal of Counselling, 26(3), 1-15.

Cornforth, S., \& Claiborne, L. (2008). Supervision in educational contexts: Raising 
the stakes in a global world. Teaching in Higher Education, 13(6), 691-701.

Crow, L. (2013). Lying down anyhow: An autoethnography. Qualitative Inquiry, 20(3), 359-361. http://doi.org/10.1177/1077800413489531

Curtis, S. (2012). Music therapy and social justice: A personal journey. The Arts in Psychotherapy, 39, 209-213.

Danaher, G., Schirato, T., \& Webb, J. (2000). Understanding Foucault. Great Britain: Sage Publications, Inc.

Daveson, B. (2008). A description of a music therapy meta-model in neuro-disability and neuro-rehabilitation for use with children, adolescents and adults. Australian Journal of Music Therapy, 19, 70-85.

Davies, B. (1991). The concept of agency: A feminist poststructuralist analysis. Social Analysis: The International Journal of Social and Cultural Practice, 30, 42-53.

Davies, B., \& Harré, R. (1990). Positioning: The discursive production of selves. Journal for the Theory of Social Behaviour, 20(1), 43-63. http://doi.org/10.1111/j.1468-5914.1990.tb00174.x

Davies, B., \& Whitehouse, H. (1997). Men on the boundaries: Landscapes and seascapes. Journal of Gender Studies, 6(3), 237-254.

Davis, A. (2005). Invisible disability. Ethics, 116(1), 153-213. http://doi.org/10.1086/453151

Davis, L. (2013). Introduction: Disability, normality, and power. In L. Davis (Ed.), The disability studies reader (4th ed., pp. 1-16). New York: Routledge: Taylor and Francis Group.

Davis, W. (2003). Ira Maximilian Altshuler: Psychiatrist and pioneer music therapist. Journal of Music Therapy, XL(3), 247-263. http://doi.org/Journal-Article

De Backer, J., \& Van Camp, J. (2003). The case of Marianne: Repetition and musical form in psychosis. In Psychodynamic music therapy (pp. 273-297). Gilsum, NH: Barcelona Publishers.

De l'Etoile, S., Dachinger, C., \& Lathroum, L. (2012). The rational-scientific 
mediating model (R-SMM): A framework for scientific research in music therapy. Music Therapy Perspectives, 30(2), 130-140.

http://doi.org/10.1093/mtp/30.2.130

de Montalk. (2013). How does it hurt?: Narrating pain. Victoria University of Wellington, New Zealand.

Defenbaugh, N. (2008). "Under erasure": The absent ill body in doctor-patient dialogue. Qualitative Inquiry, 14(8), 1402-1424. http://doi.org/10.1177/1077800408322579

Delamont, S. (2009). The only honest thing: Autoethnography, reflexivity and small crises in fieldwork. Ethnography and Education, 4(1), 51-63. http://doi.org/10.1080/17457820802703507

Denzin, N. (2001). Interpretive interactionism (2nd ed.). Newbury Park, CA: Sage Publications, Inc.

Denzin, N. (2014). Interpretive autoethnography (2nd ed.). California: Sage Publications, Inc.

Devlin, K. (2018). How do I see you, and what does that mean for us? An autoethnographic study. Music Therapy Perspectives, 36(2), 234-242. http://doi.org/10.1093/mtp/miy005

Dewald, P. (1994). Countertransference issues when the therapist is ill or disabled. American Journal of Psychotherapy, 48(2), 221-230.

Diamond, J. (1998). C: Because cowards get cancer too. Great Britain: Vermilion.

Diekema, D. (1989). Metaphors, medicine, and morals. Soundings, 72(1), 17-26.

Dileo, C. (2000). Ethical thinking in music therapy. Cherry Hill, NJ: Jeffrey Books.

Donaldson, E., \& Prendergast, C. (2011). Introduction: Disability and emotion “There's no crying in disability studies!” Journal of Literary \& Cultural Disability Studies, 5(2), 129-135. http://doi.org/10.3828/jlcds.2011.11

Drewery, W., \& Monk, G. (1994). Some reflections on the therapeutic power of poststructuralism. International Journal for the Advancement of Counselling, 17, 
$303-313$.

Du Bois, W. E. B. (1972). Black reconstruction in America: An essay toward a history of the part which Black folk played in the attempt to reconstruct democracy in America, 1860-1880. New York: Atheneum.

Du Bois, W. E. B. (1999). The souls of Black Folk. New York: WW Norton \& Co.

Dunn, H. (2009). Parallel journeys: How a music therapist can travel with his client. Approaches: Music Therapy \& Special Music Education, 1(1), 10-18.

Durie, M. (1985). A Māori perspective of health. Journal of Social Science and Medicine, 20(5), 483-486.

Durie, M. (1998). Whaiora: Māori health development (2nd Ed.). Auckland, New Zealand: Oxford University Press.

Easton, A., \& Atkin, K. (2011). Medicine and patient narratives. Social Care and Neurodisability, 2(1), 33-41. http://doi.org/10.5042/scn.2011.0082

Edwards, J. (2005a). Developments and issues in music therapy research. In B. Wheeler (Ed.), Music therapy research (2nd ed, pp. 20-32). Gilsum NH: Barcelona Publishers.

Edwards, J. (2005b). Possibilities and problems for evidence-based practice in music therapy. The Arts in Psychotherapy, 32, 293-301.

Edwards, J. (2006). A reflection on the role of informants from feminist theory in the field of music therapy. In S. Hadley (Ed.), Feminist perspectives in music therapy (pp. 367-388). Gilsum, NH: Barcelona Publishers.

Edwards, J. (2015). Getting messy: Playing, and engaging the creative, within research inquiry. Journal of Music Therapy, 52(4), 437-440. http://doi.org/10.1093/jmt/thv015

Edwards, J., \& Hadley, S. (2007). Expanding music therapy practice: Incorporating the feminist frame. The Arts in Psychotherapy, 34, 199-207.

Eisenberg, N. (2012). Post-structural conceptualizations of power relationships in physiotherapy. Physiotherapy Theory and Practice, 28(6), 439-446. 
http://doi.org/10.3109/09593985.2012.692585

Elliott, D. (1995). Music matters. New York: Oxford University Press.

Ellis, C. (2004). The ethnographic I: A methodological novel about autoethnography. Oxford, UK: AltaMira Press.

Ellis, C., \& Adams, T. (2014). The purposes, practices, and principles of autoethnographic research. In P. Leavy (Ed.), The oxford handbook of qualitative research (pp. 254-276). New York: Oxford University Press.

Ellis, C., Adams, T., \& Bochner, A. (2011). Autoethnography: An overview. Forum: Qualitative Social Research, 12(1), Art. 10.

Ellis, C., \& Bochner, A. (2000). Autoethnography, personal narrative, reflexivity. In D. Norman \& L. Yvonna (Eds.), Handbook of qualitative research (2nd ed., pp. 733-768). Thousand Oaks, Calif: Sage Publications, Inc.

Ellis, C., \& Bochner, A. (2006). Analysing analytic autoethnography: An autopsy. Journal of Contemporary Ethnography, 35(4), 429-449.

Epstein, I. (2002). Using available clinical information in practice-based research: Mining for silver while dreaming of gold. Social Work in Health Care, 33(3-4), 15-32. http://doi.org/10.1300/J010v33n03

Epston, D. (2016). Re-imagining narrative therapy: A history for the future. The Journal of Systemic Therapies, 35(1), 79-87. http://doi.org/10.1521/jsyt.2016.35.1.79

Erevelles, N. (2011). Disability and difference in global contexts: Enabling a transformative body politic. New York: Palgrave McMillan.

Etherington, K. (2004). Becoming a reflexive researcher. London, UK: Jessica Kingsley Publishers.

Ettorre, E. (2005). Gender, older female bodies and autoethnography: Finding my feminist voice by telling my illness story. Women's Studies International Forum, 28, 535-546. http://doi.org/10.1016/j.wsif.2005.09.009

Fahr, A. (2006). Autoethnography: A tool for coping with chronic illness. In K. 
Helmeke \& C. Sori (Eds.), The therapist's notebook for integrating spirituality in counseling II. USA: The Haworth Press, Inc.

Fairchild, R., \& Bibb, J. (2016). Representing people in music therapy research and practice: A balancing act. Voices: A World Forum for Music Therapy, 16(3). http://doi.org/http://dx.doi.org/10.15845/voices.v16i3.878

Fairchild, R., \& Mraz, M. (2018). " Everything changed when I got those drums ": A collaborative case reflection. Voices: A World Forum for Music Therapy, 18(1). http://doi.org/https://doi.org/10.15845/voices.v18i1.957

Feldman, S., \& Tegart, G. (2003). Keep moving: Conceptions of illness and disability of middle-aged African-American women with arthritis. Women and Therapy, 26(1-2), 127-143. http://doi.org/10.1300/J015v26n01

Ferris, J. (2011). Keeping the knives sharp. In J. Bartlett, S. Black, \& M. Northen (Eds.), Beauty is a verb: The new poetry of disability (pp. 89-93). Texas: Cinco Puntos Press.

Fisher, J. (2007). No search, no subject? Autism and the American conversion narrative. In M. Olsen (Ed.), Autism and representation (pp. 51-64). London, UK: Routledge.

Fleischman, S. (1999). I am..., I have..., I suffer from...: A linguist reflects on the language of illness. Journal of Medical Humanities, 20(1), 3-32.

Foster, E. (2013). Values and the transformation of medical education: The promise of autoethnographic research. Journal of Medicine and the Person, 11, 19-23.

Foucault, M. (1980). Power/knowledge: Selected interviews and other writings 19721977. (C. Gordon, Ed.). Sussex, UK: The Harvester Press.

Foucault, M. (1982). The Subject and Power. Critical Inquiry, 8(4), 777-795.

Foucault, M. (1984a). On the genealogy of ethics: An overview of work in progress. In P. Rabinow (Ed.), The Foucault reader: An introduction to Foucault's thought (pp. 340-372). New York: Pantheon Books.

Foucault, M. (1984b). What is enlightenment. In P. Rabinow (Ed.), The Foucault 
reader: An introduction to Foucault's thought (pp. 32-50). New York: Pantheon Books.

Foucault, M. (1997a). Michel Foucault: An interview by Stephen Riggins. In P. Rabinow (Ed.), Ethics: subjectivity and truth (essential works of Foucault 19541984 (pp. 121-133). New York: The New Press.

Foucault, M. (1997b). The ethics of the concern for the self as practice of freedom. In P. Rabinow (Ed.), Ethics, subjectivity and truth by Michel Foucault (pp. 281301). New York: Penguin Press.

Foucault, M. (2003). The birth of the clinic: An Archaeology of medical perception. ( translated-A. Sheridan, Ed.). London, UK: Routledge: Taylor and Francis Group.

Foucault, M. (2010). The government of the self and others: Lectures at the Collège de France 1982-1983. (A. Davidson \& translator-G. Burchell, Eds.). New York: Palgrave MacMillan. http://doi.org/10.1057/9780230274730

Frank, A. (1995). The wounded storyteller: Body, illness, and ethics. Chicago: The University of Chicago Press.

Frank, A. (2004). The renewal of generosity: Illness, medicine, and how to live. Chicago: The University of Chicago Press.

Fraser, M. (2007). A literate dyslexic. Hastings Women's Law Journal, 18(2), 223228.

Freeman, D. (2018). Use of reflexive thinking in music therapy to understand the therapeutic process; moments of clarity, self-Criticism: An autoethnography. Qualitative Inquiries in Music Therapy, 13(1), 0-43.

Gallardo, H., Furman, R., \& Kulkarni, S. (2009). Explorations of depression: Poetry and narrative in autoethnographic qualitative research. Qualitative Social Work, $8(3), 287-304$.

Garland-Thomson, R. (1997). Extraordinary bodies: Figuring physical disability in American culture and literature. New York: Columbia University Press.

Garland-Thomson, R. (2005). Feminist disability studies. Signs, 30(2), 1557-1587. 
Garland-Thomson, R. (2011). Misfits: A feminist materialist disability concept. Hypatia, 26(3), 591-609.

Garland-Thomson, R. (2013). Integrating disability, transforming feminist theory. In L. Davis (Ed.), The disability studies reader (4th ed., pp. 333-353). New York: Routledge: Taylor and Francis Group.

Gary, D., \& Hahn, H. (1997). Achieving occupational goals: The social effects of stigma. In C. Christiansen \& C. Baum (Eds.), Occupational therapy: Enabling function and well-being. Thorofare, NJ: Slack.

Geertz, C. (1973). The interpretation of cultures. London: Fontana Press.

Gerge, A., Wärja, M., \& Pedersen, I. (2017). Using aesthetic response - A poetic inquiry to expand knowing, Part II: Theoretical perspectives on arts-based research. Voices: A World Forum for Music Therapy, 17(1). http://doi.org/https://doi.org/10.15845/voices.v17i1.913

Gilbertson, S. (2015). In visible hands: The matter and making of music therapy. Journal of Music Therapy, 52(4), 487-514. http://doi.org/10.1093/jmt/thv014

Gill, C. (1997). Four types of integration in disability identity development. Journal of Vocational Rehabilitation, 9, 39-46.

Gill, C. (2001). Divided understandings: The social experience of disability. In G. Albrecht, K. Seelman, \& M. Bury (Eds.), Handbook of disabilities studies (pp. 351-372). Thousand Oaks, CA: Sage Publications, Inc.

Goffman, E. (1963). Stigma: Notes on the management of spoiled identities. New York: Simon \& Schuster.

Goldberg, M., Hadas-Lidor, N., \& Karnieli-Miller, O. (2014). From patient to therapatient: Social work students coping with mental illness. Qualitative Health Research, 1-12. http://doi.org/10.1177/1049732314553990

Gooding, L. (2011). The effect of a music therapy social skills training program on improving social competence in children and adolescents with social skills deficits. Journal of Music Therapy, 48(4), 440-462. 
Goodley, D. (2010). Disability Studies: An Interdisciplinary Approach. London, UK: Sage Publications, Inc.

Goodley, D., Lawthom, R., \& Runswick-Cole, K. (2014). Post-human disability studies. Subjectivity, 7, 342-361.

Goodley, D., \& Runswick-Cole, K. (2014). Becoming dishuman: Thinking about the human through dis/ability. Discourse: Studies in the Cultural Politics of Education, 37(1), 1-15. http://doi.org/10.1080/01596306.2014.930021

Gordon, C. (1991). Governmental rationality. In G. Burchell, C. Gordon, \& P. Miller (Eds.), The Foucault effect: Studies in governmentality (pp. 1-52). Hertfordshire, Great Britain: Harvester Wheatsheaf.

Gordon, I. (Ed.). (1982). The new Collins concise English dictionary (New Zealan). Collins.

Grant, A. (2011). A critique of the representation of human suffering in the cognitive behavioural therapy literature with implications for mental health nursing practice. Journal of Psychiatric and Mental Health Nursing, 18, 35-40. http://doi.org/10.1111/j.1365-2850.2010.01623.x

Grant, A., Short, N., \& Turner, L. (2013). Contemporary British Autoethnography. Rotterdam, The Netherlands: Sense Publishers.

Green, S., Davis, C., Karshmer, E., \& Marsh, P. (2005). Living stigma: The impact of labeling, stereotyping, separation, status loss, and discrimination in the lives of individuals with disabilities and their families. Sociological Inquiry, 75(2), 197215.

Grocke, D. (2016). Receptive music therapy. In J. Edwards (Ed.), The oxford handbook of music therapy (pp. 684-708). Oxford, UK: Oxford University Press.

Gross, R. (2018). The social model of disability and music therapy: Practical suggestions for the emerging clinical practitioner. Voices:A World Forum of Music Therapy, 18(1).

Hadley, S. (2001). Exploring relationships between Mary Priestley's life and work. 
Nordic Journal of Music Therapy, 10(2), 116-131.

http://doi.org/10.1080/08098130109478026

Hadley, S. (2003). Meaning making through narrative inquiry. Nordic Journal of Music Therapy, 12(1), 33-53. http://doi.org/10.1080/08098130309478071

Hadley, S. (Ed.). (2006). Feminist perspectives in music therapy. United States of America: Barcelona Publishers.

Hadley, S. (2013a). Dominant narratives: Complicity and the need for vigilance in the creative arts therapies. The Arts in Psychotherapy, 40(4), 373-381. http://doi.org/10.1016/j.aip.2013.05.007

Hadley, S. (2013b). Introduction: Let's talk about race. In S. Hadley (Ed.), Experiencing race as a music therapist: Personal narratives (pp. 3-16). Gilsum NH: Barcelona Publishers.

Hadley, S. (2014). Shifting frames: Are we really embracing human diversities? Voices: A World Forum for Music Therapy, 14(3). http://doi.org/https://voices.no/index.php/voices/article/view/801/666

Hadley, S. (2017). I don't see you as Black/Gay/Disabled/Muslim/etc.: Microaggressions in everyday encounters. In A. Whitehead-Pleaux \& X. Tan (Eds.), Cultural intersections in music therapy: Music, health, and the person. Dallas: Barcelona Publishers.

Hadley, S., \& Cooke, A. (Eds.). (n.d.). Postcolonial music therapy (in press).

Hadley, S., \& Norris, M. (2016). Musical multicultural competency in music therapy: The first step. Music Therapy Perspectives, 34(2), 129-137. http://doi.org/10.1093/mtp/miv045

Hadley, S., \& Thomas, N. (2018). Critical humanism in music therapy: Imagining the possibilities. Music Therapy Perspectives, 36(1). http://doi.org/10.1093/mtp/miy015

Hahna, N. (2017). Reflecting on personal bias. In A. Whitehead-Pleaux \& X. Tan (Eds.), Cultural intersections in music therapy: Music, health, and the person. Dallas: Barcelona Publishers. 
Hahna, N., \& Schwantes, M. (2011). Feminist music therapy pedagogy: A survey of music therapy educators. Journal of Music Therapy, 48(3), 289-316. http://doi.org/10.1093/jmt/48.3.289

Halifax, N. (2009). Disability and illness in arts-informed research: Moving toward postconventional representations. New york: Cambria Press.

Hanisch, C. (1970). “The personal is political.” In P. Allen \& S. Fire-Stone (Eds.), Notes from the second year: Women's liberation. New York: New York Radical Feminists.

Hanson-Abromeit, D. (2015). A conceptual methodology to define the therapeutic function of music. Music Therapy Perspectives, 33(1), 25-38. http://doi.org/10.1093/mtp/miu061

Harré, R. (2012). Positioning theory: Moral dimensions of social-cultural psychology. In J. Valsiner (Ed.), The oxford handbook of culture and psychology (pp. 191206). New York: Oxford University Press.

Headlam, D. (2006). Learning to hear autistically. In N. Lerner \& J. Straus (Eds.), Sounding Off: Theorizing Disability in Music (pp. 109-120). New York: Routledge.

Hershey, L. (2011). Telling. In J. Bartlett, S. Black, \& M. Northen (Eds.), Beauty is a verb: The new poetry of disability (pp. 134-135). Texas: Cinco Puntos Press.

Hodge, N. (2013). Counselling, autism and the problem of empathy. British Journal of Guidance and Counselling, 41(2), 105-116. http://doi.org/10.1080/03069885.2012.705817

Hodgson, N. (2014). He oro hauora: How do kaupapa Māori models of health relate to my music therapy practice in an adolescent acute mental health unit? Massey University and Victoria University of Wellington.

Hogue, C. (2011). The creature within: On poetry and dis/ability. In J. Bartlett, S. Black, \& M. Northen (Eds.), Beauty is a verb: The new poetry of disability (pp. 206-308). Texas: Cinco Puntos Press.

Honeyman, S. (2014). Pain Proxies, migraine and invisible disability in Renee 
French's H day. Studies in Comics, 5(2), 293-318.

Honisch, S. (2014). “Music...to cure or disable:”Therapy for whom? Voices: A World Forum for Music Therapy, 14(3), Oct. http://doi.org/10.15845/voices.v14i3.793

Hook, D. (2005). Genealogy, discourse, "effective history": Foucault and the work of critique. Qualitative Research in Psychology, 2, 3-31. http://doi.org/10.1191/1478088705qp025oa

Hooper, J., Wigram, T., Carson, D., \& Lindsay, B. (2008). A review of the music and intellectual disability literature (1943-2006) part one - descriptive philosophical writing. Music Therapy Perspectives, 26(2), 66-79.

Hoppes, S., Hamilton, T. B., \& Robinson, C. (2007). A course in autoethnography: Fostering reflective practitioners in occupational therapy. Occupational Therapy in Health Care, 21(1), 133-143.

Hoskyns, S. (2013). Enabling the curious practitioner: Perceptions on the integration of research and practice in the education of music therapy students at masters' level (unpublished doctoral thesis). New Zealand School of Music, Wellington, New Zealand.

Hoskyns, S. (2016). Book Review: Gary Ansdell, How music helps in music therapy and everyday life, from the series music therapy and everyday life. British Journal of Music Therapy, 30(2), 96-99. http://doi.org/10.1177/1359457516669099

Hoskyns, S. (2017). The place of improvisation in case study research in music therapy. The New Zealand Journal of Music Therapy, 15, 9-36.

Howe, B., \& Jensen-Moulton, S. (2016). On the disability aesthetics of music. Journal of the American Musicological Society, 69(2), 525-563.

Howe, B., Jensen-Moulton, S., Lerner, N., \& Straus, J. (2015). Introduction: Disability studies in music, music in disability studies. In B. Howe, S. Jensenmoulton, N. Lerner, \& J. Straus (Eds.), The Oxford handbook of music and disability studies (pp. 1-14). Oxford UK: Oxford University Press.

Humansim. (2019). In Encyclopaedia Britannica Online. Retrieved from 
https://www.britannica.com/topic/humanism

Humpal, M. (2017). The culture of disability. In A. Whitehead-Pleaux \& X. Tan (Eds.), Cultural intersections in music therapy: Music, health, and the person. Dallas: Barcelona Publishers.

Humphrey, J. C. (2000). Researching disability politics, or, some problems with the social model in practice. Disability \& Society, 15(January), 63-86. http://doi.org/10.1080/09687590025775

Inkle, K. (2010). Telling tales? Using ethnographic fictions to speak embodied "truth." Qualitative Research, 10(1), 27-47. http://doi.org/10. $1177 / 1468794109348681$

Jackson, S. (2001). Presidential address: The wounded healer. Bulletin of the History of Medicine, 75(1), 1-36.

Jean-Dominique. (1997). The diving bell and the butterfly. (J. Leggatt, Ed.) (trans). New York: Vintage Books.

Johnston, C., Smethurst, N., \& Gowers, S. (2005). Should people with a history of an eating disorder work as eating disorder therapists? European Eating Disorders Review, 13, 301-310. http://doi.org/10.1002/erv.659

Jones, K. A. (2012). Developing the therapeutic use of self in the health care professional through autoethnography: Working with the borderline personality disorder population. International Journal of Qualitative Methods, 11(5), 573584.

Kaiser, M. (2017). An exploration of creative arts-based self-care practices amoung music therapy students. Molloy College, Rockville Centre. Retrieved from ttps://search-proquestcom.helicon.vuw.ac.nz/docview/1951782469?accountid=14782

Kamler, B. (2001). Relocating the personal: A critical writing pedagogy. Albany: State University of New York Press.

Kay, C. (1999). Professing to care: A personal archaeology and genealogy (unpublished doctoral dissertation). Graduate School of Vanderbilt University. 
Kaye, N. (2017). Speech to National Cross Sector Forum - \$1.5 billion budget boost for education. Wellington: Beehive.govt.nz. Retrieved from https://www.beehive.govt.nz/speech/speech-national-cross-sector-forum---15billion-budget-boost-education

Keats, J. (1899). The complete poetical works and letters of John Keats, Cambridge edition. Houghton: Mifflin and Company.

Kennelly, J. (1999). Don't give up: Providing music therapy to an adolescent boy in the bone marrow transplant unit. In R. Pratt \& D. Crocke (Eds.), Music medicine (pp. 228-235). Melbourne, Australia: University of Melbourne Press.

Kenny, C. (2006). Music and life in the field of play: An anthology. Gilsum, NH: Barcelona Publishers.

Kenny, C. (2014). The field of play: An ecology of being in music therapy. Voices: $A$ World Forum for Music Therapy, 14(1). http://doi.org/http://dx.doi.org/10.15845/voices.v14i1.737

Kenny, C. (2015). Performing theory: Playing in the music therapy discourse. Journal of Music Therapy, 52(2), 457-486. http://doi.org/10.1093/jmt/thv019

Kern, P. (2011). Evidence-based practice in early childhood music therapy: A decision-making process. Music Therapy Perspectives, 29(2), 116-123.

Kidd, J., \& Finlayson, M. (2010). Mental illness in the nursing workplace: A collective autoethnography. Contemporary Nurse, 36(1-2), 21-33. http://doi.org/10.5172/conu.2010.36.1-2.021

Kielhofner, G. (2005). Rethinking disability and what to do about it: Disability studies and its implications for occupational therapy. The American Journal of Occupational Therapy, 59(5).

Klaette, J. (2018). Musikk+: En autoetnografisk layered account om musikkopplevelser i musikkterapi (unpublished masters thesis). Universitetet $\mathrm{i}$ Bergen.

Korzon, J. (2012). Fit to practice. Exploring the work experiences of registered nurses who are disabled during the course of their careers (unpublished masters 
thesis). Massey University, Manawatu, New Zealand.

Kuppers, P., \& Marcus, N. (2008). Cripple Poetics: A Love Story. Ypsilanti, Michigan: Homofactus Press.

Kwaitek, E., McKenize, K., \& Loads, D. (2005). Self-awareness and reflection: Exploring the "therapeutic use of self." Learning Disability Practice, 8(3), 2731.

Kwak, E., \& Kim, S. (2013). The use of rhythmic auditory stimulation in gait habilitation for children with cerebral palsy. Music Therapy Perspectives, 31(1), $78-83$.

Lacey, P. (2010). Smart and scruffy targets. Birmingham: The University of Birmingham,. Retrieved from https://www.google.co.nz/search?q=Smart+and+scruffy+targets\&rlz=1C5CHFA _enNZ772NZ772\&oq=Smart+and+scruffy+targets\&aqs=chrome..69i57.1091j0j $9 \&$ sourceid $=$ chrome $\&$ ie $=\mathrm{UTF}-8$

LaCom, C., \& Reed, R. (2014). Destabilizing bodies, destabilizing disciplines: Practicing liminality in music therapy. Voices: A World Forum for Music Therapy, 14(3). http://doi.org/http://dx.doi.org/10.15845/voices.v14i3.797

LaGasse, A., \& Hardy, M. (2013). Considering rhythm for sensorimotor regulation in children with autism spectrum disorders. Music Therapy Perspectives, 31(1), 6777.

Lamb, K. (1996). The problem of defining nature first: A philosophical critique of environmental ethics. The Social Science Journal, 33(4), 475-486. http://doi.org/10.1016/S0362-3319(96)90019-1

Langan, D. (2009). A music therapy assessment tool for speeial education: Incorporating education outcomes. Australian Journal of Music Therapy, 20.

Lather, P. (2006). Paradigm proliferation as a good thing to think with: Teaching research in education as a wild profusion. International Journal of Qualitative Studies in Education, 19(1), 35-57. http://doi.org/10.1080/09518390500450144

Layman, D., Hussey, D., \& Reed, A. (2013). The Beech Brook group therapy 
assessment tool: A pilot study. Journal of Music Therapy, 50(3), 155-175.

Ledger, A., \& Edwards, J. (2011). Arts-based research practices in music therapy research: Existing and potential developments. The Arts in Psychotherapy, 38, 312-317. http://doi.org/10.1016/j.aip.2011.09.001

Ledger, A., \& McCaffrey, T. (2015). Performative, arts-based, or arts-informed? Reflections on the development of arts-based research in music therapy. Journal of Music Therapy, 52(4), 441-456. http://doi.org/10.1093/jmt/thv013

Lee, C. (2003). The architecture of aesthetic music therapy. Gilsum NH: Barcelona Publishers.

Lee, K. (2010). An autoethnography: Music therapy after laser eye surgery. Qualitative Inquiry, 16(4), 244-248. http://doi.org/10.1177/1077800409351975

Lennard, N., \& Wolfe, C. (2017, January). Is humanism really humane? The New York Times. Retrieved from https://www.nytimes.com/2017/01/09/opinion/ishumanism-really-humane.html

Leon, R. (2004). Group psychotherapy and Parkinson's Disease: When members and therapist share the diagnosis. International Journal of Group Psychotherapy, 54(3), 401-409.

Lerner, N., \& Straus, J. (Eds.). (2006). Sounding off: Theorizing disability in music. New York: Routledge.

Levine, S. (2013). Expecting the unexpected: Improvisation in arts-based research. Journal of Applied Arts and Health, 4(1), 21-28.

Levinson, F., \& Parritt, S. (2006). Against stereotypes: Experiences of disabled psychologists. In D. Goodley \& R. Lawthom (Eds.), Disability and psychology: Critical introductions and reflections (pp. 111-122). New York: Palgrave MacMillan.

Lewis, G. (2017). "Let your secrets sing out": An auto-ethnographic analysis on how music can afford recovery from child abuse. Voices: A World Forum for Music Therapy, 17(2). 
Lim, H. (2010a). Effect of "developmental speech and language training through music" on speech production in children with autism spectrum disorders. Journal of Music Therapy, 47(1), 2-26. http://doi.org/10.1093/jmt/47.1.2

Lim, H. (2010b). Use of music in the applied behavior analysis verbal behavior approach for children with autism spectrum disorders. Music Therapy Perspectives, 28(2), 95-105.

Lindsey, E. (1996). Health within illness: Experiences of chronically ill/disabled people. Joumal of Advanced Nursing, 24, 465-472.

Ling, H. K. (2012). Diversity in disability studies: A Malaysian perspective. In Disability studies: Every body in. Dunedin: Otago University, New Zealand.

Link, Bruce, \& Phelan. (2001). Conceptualizing stigma. Annual Review of Sociology, $27,363-385$.

Lipski, G. (2014). Finding where I fit: An autoethnography on integrating ethnomusicology into music therapy (unpublished masters thesis). Concordia University.

Long, L. (2008). Narrative autoethnography and the promotion of spiritual well-being in teacher research and practice. Pastoral Care in Education, 26(3), 187-196. http://doi.org/10.1080/02643940802246575

Longmore, P. (2013). "Heaven's special children" The making of poster children. In L. Davis (Ed.), The disability studies reader (4th ed., pp. 34-41). New York: Routledge: Taylor and Francis Group.

Lubet, A. (2011). Music, disability, and society. Philadelphia: Temple University Press.

Mackay, H. (2013). The goodlife: What makes life worth living. Australia: Pam Macmillan Australia.

Madge, C. (2010). Key competencies success rubics. New Zealand: Learning with Zeal: Empowering kids in a New Zealand School.

Magee, W. (2011). Music technology for health and wellbeing: The bridge between 
the arts and science. Music and Medicine, 3(3), 131-133.

Magee, W. (2017). Music-making in therapeautic contexts. In R. MacDonald, D. Hargreaves, \& D. Miell (Eds.), Handbook of musical identities. Oxford UK: Oxford Scholarship Online. http://doi.org/10.1093/acprof

Magee, W. (2018). Developing theory for using music technologies in music therapy. Nordic Journal of Music Therapy, 27(5), 334-336. http://doi.org/10.1080/08098131.2018.1481450

Maggio, L. M. (2007). Externalizing lupus: A therapist/patient's challenge. Professional Psychology: Research and Practice, 38(6), 576-581. http://doi.org/10.1037/0735-7028.38.6.576

Mahoney, E. (2015). Multicultural music therapy: An exploration. Voices: A World Forum for Music Therapy, 15(2). http://doi.org/http://dx.doi.org/10.15845/voices.v15i2.844

Manathunga, C. (2007). "Unhomely" academic developer identities: More postcolonial explorations. International Journal for Academic Development, 12(1), 25-34. http://doi.org/10.1080/13601440701217287

Manovski, M. (2014). Arts-based research, autoethnography, and music education: Singing through a culture of marginalisation. Rotterdam, The Netherlands: Sense Publishers.

Matthews, N. (2009). Teaching the "invisible" disabled students in the classroom: disclosure, inclusion and the social model of disability. Teaching in Higher Education, 14(2), 229-239. http://doi.org/10.1080/13562510902898809

McCaffrey, T., \& Edwards, J. (2015). Meeting art with art: Arts-based methods enhance researcher reflexivity in research with mental health service users. Journal of Music Therapy, 52(4), 515-532. http://doi.org/10.1093/jmt/thv016

McCullough, L. (1989). The abstract character and transforming power of medical language. An Interdisciplinary Journal, 72(1), 111-125.

McCurdy, L., Winterbottom, K., Mehta, S., \& Roberts, J. (2010). Using nature and outdoor activity to improve children's health. Current Problems in Pediatric and 
Adolescent Health Care, 40(5), 102-117.

http://doi.org/10.1016/j.cppeds.2010.02.003

McDermott, O., Orrell, M., \& Ridder, H. M. (2014). The importance of music for people with dementia: The perspectives of people with dementia, family carers, staff and music therapists. Aging and Mental Health, 18(6), 706-716. http://doi.org/10.1080/13607863.2013.875124

McFerran, K. (2012). Who is my “client." Voices Resources. Retrieved from http://testvoices.uib.no/community/?q=fortnightly-columns/2012-who-my-client

McFerran, K. (2017a). An inclusive stance with a critical edge. Voices: A World Forum for Music Therapy, 17(2), 1-2. http://doi.org/https://dx.doi.org/10.15845/voices.v17i2.944

McFerran, K. (2017b). "I would die without my music": Relying on musical identities to cope with difficult times. In R. MacDonald, D. Hargreaves, \& D. Miell (Eds.), Handbook of musical identities. Oxford UK: Oxford Scholarship Online. http://doi.org/10.1093/acprof

McFerran, K. (2018). Critical thinking in disability and mental health: Where did it come from and what does it mean for music therapy practice? In Music Therapy online. Retrieved from https://musictherapyonline.org/p/critical-thinking

McGrath-Smith, E. (2011). Afraid of the rake. In J. Bartlett, S. Black, \& M. Northen (Eds.), Beauty is a verb: The new poetry of disability (p. 286). Texas: Cinco Puntos Press.

McGraw Hunt, A. (2016). First person research. In B. Wheeler \& K. Murphy (Eds.), Music therapy research (3rd ed., pp. 443-467). Dellas: Barcelona Publishers.

McIlveen, P. (2007). The genuine scientist-practitioner in vocational psychology: An autoethnography. Qualitative Research in Psychology, 4, 295-311. http://doi.org/10.1080/14780880701522403

McIlveen, P. (2008). Autoethnography as a method for reflexive research and practice in vocational psychology. Australian Journal of Career Development, 17(2), 1321. 
McIlveen, P., Beccaria, G., du Preez, J., \& Patton, W. (2010). Autoethnography in vocational psychology: Wearing your class on your sleeve. Journal of Career Development, 37(3), 599-615.

McIntosh, P. (1988). White privilege and male privilege: A personal account of coming to see correspondences through work in women's studies (working paper no.189) (No. 189). Wellesley, MA.

McKay, G. (2009). “Crippled with nerves”: Popular music and polio, with particular reference to Ian Dury. Popular Music, 28(3), 341-365. http://doi.org/10.1017/S0261143009990109

McKay, G. (2013a). Introduction: Cultural disability studies and the cripping and popping of theory. In Shakin' all over: Popular music and disability (pp. 1-18). Michigan: University of Michigan Press.

McKay, G. (2013b). Shakin' all over: Popular music and disability. Michigan: University of Michigan Press.

McNiff, S. (1998). Art-based Research. London: Jessica Kingsley Publishers.

McRuer, R. (2006). Crip theory: Cultural signs of queerness and disability. New York: New York University Press. http://doi.org/10.1017/CBO9781107415324.004

Metell, M. (2014). Dis/abling musicking: Reflections on a disability studies perspective in music therapy. Voices: A World Forum for Music Therapy, 14(3).

Metell, M., \& Stige, B. (2015). Blind spots in music therapy. Toward a critical notion of participation in context of children with visual impairment. Nordic Journal of Music Therapy, 25(4), 300-318. http://doi.org/10.1080/08098131.2015.1081265

Ministry of Education. (2009a). Career education and guidance in New Zealand schools. Wellington. Retrieved from file://Users/carolynayson/Downloads/Career-guidelines-web.pdf

Ministry of Education. (2009b). Narrative assessment: A guide for teachers. Wellington: Learning Media Limited. 
Ministry of Education. (2012). Special education initiatives. New Zealand. Retrieved from https://education.govt.nz/ministry-of-education/budgets/budget2012/special-education-initiatives/

Ministry of Education. (2013). Specialist service standards: Question list for specialist. New Zealand.

Ministry of Education. (2019). Key competencies. Retrieved January 5, 2019, from http://nzcurriculum.tki.org.nz/Key-competencies

Ministry of Health. (2001). The New Zealand Disability Strategy: Making a world of difference, Whakanui Oranga. Wellington.

Mitra, S. (2006). The capability approach and disability. Journal of Disability Policy Studies, 16(4), 236-247. http://doi.org/10.1177/10442073060160040501

Moir, J. (2016, August). Special-needs funding for children over seven "slashed" under new govt proposal. Stuff. Wellington. Retrieved from https://www.stuff.co.nz/national/education/83429927/specialneeds-funding-forchildren-over-seven-slashed-under-new-govt-proposal

Moore, I. S. (2013). “The beast within": Life with an invisible chronic illness. Qualitative Inquiry, 19(3), 201-208. http://doi.org/10.1177/1077800412466052

Morella-Pozzi, D. (2014). The (dis)ability double life. In R. Boylorn \& M. Orbe (Eds.), Critical autoethnography: Intersecting cultural identities in everyday life (pp. 176-188). Walnut Creek, CA: Left Coast Press, Inc.

Morgan, A. (2000). What is narrative therapy?: An easy to read introduction. Adelaide, Australia: Dulwich Centre Publications.

Morgan, R. (2015). Four powerful poems about Parkinson's and growing older. America: TED Talk. Retrieved from https://www.youtube.com/watch?v=j97WsAz3CDY\&list=PL1wPM0Yrsx8jV8cpIvcZd5Gwg8Cgsz27\&index $=6$

Morrison, A. L. (1997). Ten years of doing psychotherapy while living with a lifethreatening illness self-disclosure and other ramifications. Psychoanalytic Dialogues, 7(2), 225-241. http://doi.org/10.1080/10481889709539178 
Mouffe, C. (2016). Democratic politics and conflict: An agonistic approach. Politica Común, 9.

Mullay, B. (2001). Confronting the politics of despair: Toward the reconstruction of progressive social work in a global economy and postmodern age. Social Work Education, 20(3), 303-320.

Music Therapy New Zealand. (2012). Code of ethics for the practice of music therapy in New Zealand. Retrieved from https://www.musictherapy.org.nz/documents/Code-of-Ethics-2012.pdf

Nabors, N., \& Pettee, M. (2003). Womanist therapy with African American women with disabilities. Women and Therapy, 26(3-4), 221-241. http://doi.org/10.1300/J015v26n03

Nachmanovitch. (1991). Free play: Improvisation in life and art. New York: Penguin Putnam Inc.

Neal-Boylan, L. (2012). An exploration and comparison of the worklife experiences of registered nurses and physicians with permanent physical and/or sensory disabilities. Rehabilitation Nursing, 37(1), 3-10.

Neville-Jan, A. (2003). Encounters in a world of pain: An autoethnography. American Journal of Occupational Therapy, 57, 88-98.

Neville-Jan, A. (2004). Selling your soul to the devil: An autoethnography of pain, pleasure and the quest for a child. Disability \& Society, 19(2), 113-127. http://doi.org/10.1080/0968759042000181758

New Zealand Music Therapy Registration Board. (2012). Music therapy practice in New Zealand. Retrieved from https://www.musictherapy.org.nz/documents/Registered-Music-TherapistStandards-of-Practice-2012.pdf

New Zealand School of Music. (2013). NZSM 451 Paper outline. Wellington.

Nordoff, P., \& Robbins, C. (2007). Creative music therapy: A guide to fostering clinical musicianship (2nd ed.). Gilsum, NH: Barcelona Publishers. 
O'Brian, L. (2002). She bop II: The definitive history of women in rock, pop and soul. London, UK: Continuum.

O’Callaghan, C. (2009). Practice informed research in oncologic and palliative music therapy: From clinical data-mining to RCT. Australian Journal of Music Therapy, 20(special issue).

O’Grady, L., \& McFerran, K. (2006). Birthing feminist community music therapy. In S. Hadley (Ed.), Feminist perspectives in music therapy (pp. 63-80). Gilsum, NH: Barcelona Publishers.

Oliver, M. (1996). Defining impairement and disability: Issues at stake. In C. Barnes \& G. Mercer (Eds.), Exploring the divide (pp. 29-54). Leeds: The Disability Press.

Olney, M., \& Brockelman, K. (2003). Out of the disability closet: Strategic use of perception management by select university students with disabilities. Disability \& Society, 18(June), 35-50. http://doi.org/10.1080/713662200

Oswanski, L., \& Donnenwerth, A. (2017). Allies for social justice. In A. WhiteheadPleaux \& X. Tan (Eds.), Cultural intersections in music therapy: Music, health, and the person. Dallas: Barcelona Publishers.

Oxford Dictionaries. (2018). English Oxford Living Dictionaries. Oxford University Press. Retrieved from https://en.oxforddictionaries.com

Packer, M. (2011). The science of qualitative research. Cambridge, UK: Cambridge University Press.

Pafunda, D. (2011). Meat life. In J. Bartlett, S. Black, \& M. Northen (Eds.), Beauty is a verb: The new poetry of disability (pp. 313-314). Texas: Cinco Puntos Press.

Parse, R. (1990). Health a personal commitment. Nursing Science Quarterly, 3, 136140.

Patston, P. (2015, October). Disabled people: Toughen up, gently. Diversity NZ. Retrieved from http://www.philippatston.com/blog/disabled-people-toughen-upgently/ 
Pavlicevic, M. (1997). Music therapy in context: Music, meaning and relationship. London: Jessica Kingsley Publishers.

Pavlicevic, M., \& Ansdell, G. (2004). Introduction: “The ripple effect.” In M. Pavlicevic \& G. Ansdell (Eds.), Community music therapy (pp. 15-31). London: Jessica Kingsley Publishers.

Pedersen, H. (2011). Counting affects: Mo(ve)ments of intensity in critical avian education. Canadian Journal of Environmental Education, 16, 14-28.

Peile, C. (1988). Research paradigms in social work: From stalemate to creative synthesis. Social Service Review, 62(1), 1-19.

Peterson, C. (2018). Monkey trouble: The scandal of posthumanism. New York: Fordham University Press.

Pfeiffer, D. (2001). The conceptualization of disability. In B. Altman \& S. Barnartt (Eds.), Exploring theories and expanding methodologies (pp. 29-52). Oxford UK: Elsevier Ltd.

Phillips, B. (2007). Nursing care and understanding the experiences of others: A Gadamerian perspective. Nursing Inquiry, 14(1), 89-94.

Poulin, C., \& Gouliquer, L. (2003). Part-time disabled lesbian passing on roller blades, or PMS, prozac, and essentializing women's ailments. Women and Therapy, 26(1-2), 95-108. http://doi.org/10.1300/J015v26n01

Priestley, M. (1994). Essays on analytic music therapy. Gilsum, NH: Barcelona Publishers.

Prizant, B. (2015). Uniquely human: A different way of seeing autism. New York: Simon \& Schuster.

Puar, J. (2013). The cost of getting better: Ability and debility. In L. Davis (Ed.), The disability studies reader (4th ed., pp. 177-184). New York: Routledge: Taylor and Francis Group.

Quinn, J. (2013). Theorising learning and nature: Post-human possibilities and problems. Gender and Education, 25(6), 738-753. 
http://doi.org/10.1080/09540253.2013.831811

Raglio, A., Traficante, D., \& Oasi, O. (2011). Autism and music therapy.

Intersubjective approach and music therapy assessment. Nordic Journal of Music Therapy, 20(2), 123-141. http://doi.org/10.1080/08098130903377399

Rance, N., Moller, N., \& Douglas, B. (2010). Eating disorder counsellors with eating disorder histories: A story of being "normal”. Eating Disorders: The Journal of Treatment and Prevention, 18, 377-392.

http://doi.org/10.1080/10640266.2010.511901

Randall, J., \& Munro, L. (2010). Foucault's care of the self: A case from mental health work. Organization Studies, 39(11), 1485-1504.

Reeve, D. (2006). Towards a psychology of disability: The emotional effects of living in a disabling society. In D. Goodley \& R. Lawthom (Eds.), Disability and psychology: Critical introductions and reflections (pp. 94-108). New York: Palgrave MacMillan.

Reeve, D. (2012). Cyborgs, cripples and iCrip: Reflections on the contribution of Haraway to disability studies. In D. Goodley, B. Hughes, \& J. Davis (Eds.), Disability and social theory: New developments and directions (pp. 91-111). London, UK: Palgrave MacMillan. http://doi.org/10.1057/9781137023001

Reynolds, F., \& Prior, S. (2003). “A lifestyle coat-hanger”: A phenomenological study of the meanings of artwork for women coping with chronic illness and disability. Disability and Rehabilitation, 25(14), 785-794. http://doi.org/10.1080/0963828031000093486

Richards, R. (2008). Writing the othered self: Autoethnography and the problem of objectification in writing about illness and disability. Qualitative Health Research, 18(12), 1717-1728. http://doi.org/10.1177/1049732308325866

Richardson, L. (2000). New writing practices in qualitative research. Sociology of Sport Journal, 17, 5-20.

Rickson, D. (2004). Angela's mother: The early years. New Zealand Journal of Music Therapy, 2, 17-35. 
Rickson, D. (2006). Instructional and improvisational models of music therapy with adolescents who have Attention Deficit Hyperactivity Disorder (ADHD): A comparison of the effects on motor impulsivity. Journal of Music Therapy, 43(1), 39-62.

Rickson, D. (2014a). Active music: Participatory action research about music for young people with intellectual disability. Paper presented at Moving forward through research. Queenstown, New Zealand.

Rickson, D. (2014b). Can therapy in education be dangerous? Voices: A World Forum for Music Therapy, 14(1). http://doi.org/http://dx.doi.org/10.15845/voices.v14i1.747

Rickson, D. (2014c). Stepping into the spotlight: Collaborative efforts towards musical participation. Approaches: Music Therapy \& Special Music Education, 6(2), 12-99.

Rickson, D. (2014d). The relevance of disability perspectives in music therapy practice with children and young people who have intellectual disability. Voices: A World Forum for Music Therapy, 14(3). http://doi.org/http://dx.doi.org/10.15845/voices.v14i3.784

Rickson, D., \& McFerran, K. (2014). Creating music cultures in the schools: A perspective from community music therapy. University Park: Barcelona Publishers.

Rickson, D., \& Twyford, K. (2011). Music therapy consultation in a New Zealand School: Staff members' perceptions of outcomes. The New Zealand Journal of Music Therapy, 9, 61-85.

Rigney, M. (2011, October 10). Using reflexive tools for coping and bringing closure in pediatric palliative care: A music therapist's story of working with a terminally ill child. Voices: A World Forum for Music Therapy. Retrieved from https://voices.no/index.php/voices/article/view/287/471

Rix, J. (2012). From equality to diversity? Ideas that keep us quiet. In J. Solar, C. Walsh, A. Craft, J. Rix, \& K. Simmons (Eds.), Transforming practice: Critical issues in equity, diversity and education (pp. 245-256). Staffordshire, UK: 
Trentham Books.

Roger, M. (2014). When being a woman is an impediment to care: Dysautonomia.

Retrieved September 2, 2018, from

https://bobisdysautonomia.blogspot.com/2014/11/when-being-woman-isimpediment-to.html

Rolvsjord, R. (2010). Resource oriented music therapy: In mental healthcare. Gilsum NH: Barcelona Publishers.

Rolvsjord, R. (2014). The competent client and the complexity of dis-ability. Voices: A World Forum for Music Therapy, 3(14).

http://doi.org/http://dx.doi.org/10.15845/voices.v14i3.787

Rolvsjord, R. (2015). Five episodes of clients' contributions to the therapeutic relationship: A qualitative study in adult mental health care. Nordic Journal of Music Therapy, 24(4), 296-321. http://doi.org/10.1080/08098131.2015.1010562

Roman, T. (2016). "Were they better today?" Valuing client's individual therapeutic process and predictable outcomes. British Journal of Music Therapy, 30(1), 1321.

Rønnestad, M., \& Skovholt, T. (2003). The journey of the counselor and therapist: Research findings and perspectives on professional development. Journal of Career Development, 30(1), 5-44.

Roscoe, L. (2012). Healing the physician's story: A case study in narrative medicine and end of life care. Narrative Inquiry in Bioethics, 2.1, 65-72.

Rose, N. (1996). Inventing ourselves: Psychology, power, and personhood. Cambridge: Cambridge University Press.

Rose, N. (2000). Identity, genealogy, history. In P. Du Gay, J. Evans, \& P. Redman (Eds.), Identity: A reader (pp. 311-324). London, UK: Sage Publications, Inc.

Ruud, E. (2005). Philosophy and theory of science. In B. Wheeler (Ed.), Music therapy research (pp. 33-44). Gilsum, NH: Barcelona Publishers.

Ruud, E. (2010). Music therapy: A perspective from the humanities. Gilsum, NH: 
Barcelona Publishers.

Rykov, M. (2011). Writing music therapy. Voices: A World Forum for Music

Therapy, 11(1). http://doi.org/http://dx.doi.org/10.15845/voices.v11i1.288

Sachs, M., Damasio, A., \& Habibi, A. (2015). The pleasures of sad music: A systematic review. Frontiers in Human Neuroscience, 9(July), 1-12. http://doi.org/10.3389/fnhum.2015.00404

Sacks, O. (2008). Musicophilia: Tales of music and the brain, revised and expanded edition. New York: Vintage Books.

Sampson, E. (2003). Unconditional kindness to strangers. Theory and Psychology, 13(2), 147-175.

Samuels, E. (2013). My body, my closet: Invisible disability and the limits of coming out. In L. Davis (Ed.), The disability studies reader (4th ed., pp. 316-332). New York: Routledge: Taylor and Francis Group.

Samuels, E. (2014a). Introduction: The crisis of identification. In Fantasies of identification (pp. 1-23). New York: NYU Press. Retrieved from http://www.jstor.org/stable/j.ctt9qfj7x.4

Samuels, E. (2014b). Proving disability. In Fantasies of identification (pp. 121-140). New York: NYU Press.

Samuels, E. (2014c). The disability con onscreen. In Fantasies of identification (pp. 66-79). New York: NYU Press. Retrieved from Your

Schenstead, R. (2012). The timelessness of arts-based research: Looking back upon a heuristic self-study and the arts-based reflexivity data analysis method. Voices: A World Forum for Music Therapy, 12(1).

Schrag. (2015). A triple insider's take on arts therapy, arts-based community development, and Huntington's Disease. Voices: A World Forum for Music Therapy, 15(3). Retrieved from https://doi.org/10.15845/voices.v15i3.822

Schuman, E. (2002). The sound of a wild snail eating. The Missouri Review, 25(2), 31-42. http://doi.org/10.1353/mis.2002.0040 
Scott, R. (1969). The making of blind men. New York: Russell Sage.

Scurry, E. (1985). The body in pain: The making and unmaking of the world. New York: Oxford University Press.

Sears, W. (2007). A re-vision and expansion of processes in music therapy. In M. Sears (Ed.), Music: The therapeatic edge: Readings from William W. Sears (pp. 16-41). Gilsum, NH: Barcelona Publishers.

Shakespeare, T. (2012). Making a difference: How disability research can change our world. In Disability studies: Everybody in. Dunedin: University of Otago, New Zealand.

Shakespeare, T. (2013). The social model of disability. In L. Davis (Ed.), The disability studies reader (4th ed., pp. 214-221). New York: Routledge: Taylor and Francis Group.

Sharpiro, J. (2012). Narrative medicine and narrative writing. Family Medicine, 5, $309-311$.

Shaw, C. (2016). Seeing the unseen: A post-structural look at disability in music therapy practice. Paper presented at Music Therapy New Zealand Hui. Auckland, New Zealand.

Siebers, T. (2008). Disability theory. Michigan: The University of Michigan Press.

Siebers, T. (2010). Disability aesthetics. Michigan: University of Michigan Press.

Siebers, T. (2013). Disability and the theory of complex embodiment - for identity politics in new register. In L. Davis (Ed.), The disability studies reader (4th ed, pp. 278-297). New York: Routledge: Taylor and Francis Group.

Siisiainen, L. (2008). From the empire of the gaze to noisy bodies: Foucault, audition and medical power. Project Muse, 11(1).

Skaife, S. (2013). Black and white: Applying Derrida to contradictory experiences in an art therapy group for victims of torture. Group Analysis, 46(3), 256-271.

Slattery, P. (2001). The educational researcher as artist working within. Qualitative Inquiry, 7(3), 370-398. 
Small, C. (1998). Musicking: The meanings of performing and listening. New England, Hanover: Wesleyan Univeristy Press.

Smith, C. (2005). Epistemological intimacy: A move to autoethnography. International Journal of Qualitative Methods, 4(2), 68-76.

Smith, C. (2012). (Re)discovering meaning: A tale of two Losses. Qualitative Inquiry, 18(10), 862-867. http://doi.org/10.1177/1077800412456962

Smith, J. (2012). Becoming-music: A description of an improvisational music therapy outreach program: International Journal of Child, Youth and Family Studies, 2 \& $3,272-283$.

Solli, H. P. (2015). Battling illness with wellness: A qualitative case study of a young rapper's experiences with music therapy. Nordic Journal of Music Therapy, 24(3), 204-231. http://doi.org/10.1080/08098131.2014.907334

Sollod, R. (2002). Beyond a sense of safety: A psychologist's tale of serious chronic illness. Journal of Clinical Psychology, 58(11), 1397-409. http://doi.org/10.1002/jclp.10087

Sontag, S. (1978). Illness as metaphor. Toronto: McGraw-Hill Ryerson Ltd.

Souza, V. (2014, February). Who's afraid of chronic illness as disability? An entire field, apparantly. It's Complicated: Writings on Disability, Academia, Architecture, and Everything in between, 9.

Spry, T. (2012). Performative autoethnography: Critical embodiments and possibilities. In N. Denzin \& Y. Lincoln (Eds.), Collecting and interpreting qualitative materials (pp. 213-244). Thousand Oaks, CA: Sage Publications, Inc.

St. Pierre, E. (2001). Coming to theory: Finding Foucault and Deleuze. In K. Weiler (Ed.), Feminist engagements: Reading, resisting, and revisioning male theorists in education and cultural studies (pp. 141-164). New York: Routledge.

St. Pierre, J. (2015). Cripping communication: Speech, disability, and exclusion in liberal humanist and posthumanist discourse. Communication Theory, 25, 330348. http://doi.org/10.1111/comt.12054 
Stace, H. (2007). The long unfinished journey towards human rights for disabled people in Aotearoa New Zealand. Human Rights Research, 5, 1-13.

Stanford Encyclopedia of Philosophy. (2011). Disability: Definitions, models, experience. Stanford. Retrieved from https://plato.stanford.edu/entries/disability/

Stanley, N., Ridley, J., Manthorpe, J., Harris, J., \& Hurst, A. (2007). Disclosing disability: Disabled students and practitioners in social work, nursing and teaching. Disability Rights Commision.

Stige, B. (2002). Culture-centered music therapy. Gilsum, NH: Barcelona Publishers.

Stige, B. (2005). Ethnography and ethnographically informed research. In B. Wheeler (Ed.), Music therapy research (2nd ed, pp. 392-403). Gilsum, NH: Barcelona Publishers.

Stige, B., \& Ledger, A. (2016). Ethnographic research. In B. Wheeler \& K. Murphy (Eds.), Music Therapy Research (3rd ed, pp. 408-420). Dallas: Barcelona Publishers.

Stone, S. D. (2005). Reactions to invisible disability: The experiences of young women survivors of hemorrhagic stroke. Disability and Rehabilitation, 27(6), 293-304. http://doi.org/10.1080/09638280400008990

Storer, J. (2014). Inhabiting transience: Stories of learning through chronic illness, the body, place and creativity (unpublished doctoral dissertation). The University of New England.

Stras, L. (2009). Sing a song of difference: Connie Boswell and a discourse of disability in jazz. Popular Music, 28(3), 297-322. http://doi.org/10.1017/S0261143009990080

Straus, J. (2006). Normalizing the abnormal: Disability in music and music theory. American Musicological Society, 59(1), 113-184.

Straus, J. (2011). Extraordinary Measures: Disability in music. Oxford UK: Oxford University Press. http://doi.org/10.1093/acprof

Straus, J. (2014). Music therapy and Autism: A view from disability studies. Voices: 
A World Forum for Music Therapy, 14(3), Sep.

http://doi.org/10.15845/voices.v14i3.785.

Sue, D. W. (2010). Microaggressions in everyday life: Race, gender, and sexual orientation. Hoboken, NJ: John Wiley \& Sons, Inc. http://doi.org/10.1177/0361684311401818

Sue, D. W., \& Sue, D. (2015). Counseling the culturally diverse: Theory and practice (7th ed.). John Wiley \& Sons, Inc.

Suzuki, D. (1992). The declaration of interdependence. Vancouver. Retrieved from https://davidsuzuki.org/about/declaration-of-interdependence/

Swain, J., \& Cameron, C. (1999). Unless otherwise stated: Discourses of labelling and identity in coming out. In M. Corker \& S. French (Eds.), Disability discourse (pp. 68-78). Philadelphia: Open University Press.

Swain, J., \& French, S. (2000). Towards an affirmation model of disability. Disability \& Society, 15, 569-582.

Swank, J., Min Shin, S., Cabrita, C., Cheung, C., \& Rivers, B. (2015). Initial investigation of nature-based, child-centered play therapy: A single-case design. Journal of Counseling and Development, 93, 440-450.

Swenson, R. (2012). I am Okay until I am measured by the perceived norms. In Disability Studies: Every body in. Dunedin: Otago University, New Zealand.

Tambakaki, P. (2014). The tasks of agonism and agonism to the task: Introducing Chantal Mouffe: Agonism and the politics of passion. Parallax. Taylor \& Francis. http://doi.org/10.1080/13534645.2014.896543

Thomas, C. (2007). Sociologies of disability and illness: Contested ideas in disability studies and medical sociology. New york: Palgrave MacMillan.

Todd, L. (2006). Enabling practice for professionals; The need for practical poststructuralist theory. In D. Goodley \& R. Lawthom (Eds.), Disability and psychology: Critical introductions and reflections (pp. 141-154). New York: Palgrave MacMillan. 
Toppozada, M. (1995). Multicultural training for music therapists: An examination of current issues based on a national survey of professional music therapists. Journal of Music Therapy, 32, 65-90. http://doi.org/doi:10.1093/mtp/24.2.108

Trondalen, G. (2016). Self-care in music therapy: The art of balancing. In J. Edwards (Ed.), The oxford handbook of music therapy. Oxford, UK: Oxford University Press.

Tsiris, G. (2013). Voices from the "ghetto": Music therapy perspectives on disability and music (A reposnse to Joseph Straus's book extraordinary measures: Disability in music). International Journal of Community Music, 6(3), 333-343.

Tsiris, G. (2016). Music therapy and spirituality: an international survey of music therapists' perceptions. Nordic Journal of Music Therapy, 26(4), 1-27. http://doi.org/10.1080/08098131.2016.1239647

Tsiris, G. (2018). A misunderstood guest, a misunderstood host: Music therapy within disability studies. Paper presented at Cripping the muse. Leeds, UK. Retrieved from https:/crippingthemuse.com/contributors/

Turner, K. (2017). "The lines between us": Exploring the identity of the community musician through an arts practice research approach. Voices: A World Forum for Music Therapy, 17(3). http://doi.org/https://dx.doi.org/10.15845/voices.v17i3.941

Twyford, K. (2012). Getting to know you: Peer and staff perceptions of involvement in inclusive music therapy groups with students with special education needs in mainstream school settings. The New Zealand Journal of Music Therapy, 10, 3973.

Uotinen, J. (2011). Senses, bodily knowledge, and autoethnography: Unbeknown knowledge from an ICU experience. Qualitative Health Research, 21(10), 13071315. http://doi.org/10.1177/1049732311413908

Vaillancourt, G. (2009). Mentoring apprentice music therapists for peace and social justice through community music therapy: An arts-based study (unpublished doctoral dissertation). Antioch University. 
Vaillancourt, G. (2011). Creating an apprenticeship music therapy model through arts-based research. Voices: A World Forum for Music Therapy, 11(1). http://doi.org/10.15845/voices.v11i1.341

Vaillancourt, G. (2012). Music therapy: A community approach to social justice. Arts in Psychotherapy, 39(3), 173-178. http://doi.org/10.1016/j.aip.2011.12.011

Vash, C. (1981). The psychology of disability. New York: Springer.

Velde, B. (1999). The experience of being an occupational therapist with a disability. The American Journal of Occupational Therapy, 54(2), 183-188.

Viega, M. (2013). "Loving me and my butterfly wings:” A study of hip-hop songs written by adolescents in music therapy (unpublished doctoral dissertation). Temple University, Philadelphia.

Viega, M. (2016a). Aesthetic sense and sensibility: Arts-Based research and music therapy. Music Therapy Perspectives, 34, 1-3. http://doi.org/10.1093/mtp/miw010

Viega, M. (2016b). Science as art: Axiology as a central component in methodology and evaluation of arts-based research (ABR). Music Therapy Perspectives, 34(845), 4-13. http://doi.org/10.1093/mtp/miv043

Viega, M. (2017). Creating, providing, and performing space. Voices: A World Forum for Music Therapy, 17(3). http://doi.org/https://dx.doi.org/10.15845/voices.v17i3.952

Viega, M., \& Forinash, M. (2016). Arts-based research. In B. Wheeler \& K. Murphy (Eds.), Music therapy research (3rd ed., pp. 491-504). Dellas: Barcelona Publishers.

Vining, J., Merrick, M., \& Price, E. (2008). The distinction between humans and nature: Human perceptions of connectedness to nature and elements of the natural and unnatural. Human Ecology Review, 15(1), 1-11.

Walker, L. (2001). Looking like what you are. New York: New York University Press. 
Walker, N. (2014). What is autism? Neurocosmopolitanism: Nick Walker's Notes on Neurodiversity, Autism, and Cognitive Liberty. Retrieved from http://neurocosmopolitanism.com/what-is-autism/

Wall, D. (2005). Encounters with the invisible: Unseen illness, controversy, and chronic fatigue syndrome. Texas: First Southern Methodist University Press.

Wall, S. (2006). An autoethnography on learning about autoethnography. International Journal of Qualitative Methods, 5(2), Article 9. Retrieved from http://www.ualberta.ca/ iiqm/backissues/5_2/pdf/wall.pdf

Warne, K., \& Hoppes, S. (2009). Lessons in living and dying from my first patient: An autoethnography. Canadian Journal of Occupational Therapy, 76(4), 309316.

Warren, P., \& Nugent, N. (2010). The music connections programme: Parents' perceptions of their children's involvement in music therapy. The New Zealand Journal of Music Therapy, 8, 8-33.

Webster, J., \& Rickson, D. (2018). Selecting the best music for patient listening while unwell: A music therapist's personal experience. The New Zealand Journal of Music Therapy, 16, 55-69.

Weise, J. (2011). From the disability rights movement and the legacy of poets with disabilities. In J. Bartlett, S. Black, \& M. Northen (Eds.), Beauty is a verb: The new poetry of disability (pp. 138-145). Texas: Cinco Puntos Press.

Wendell, S. (1997). Toward a feminist theory of disability. In L. Davis (Ed.), The disability studies reader (pp. 260-278). New York: Routledge.

Wendell, S. (2001). Unhealthy disabled: Treating chronic illnesses as disabilities. Hypatia, 16(4), 17-33. Retrieved from http://muse.jhu.edu/journals/hyp/summary/v016/16.4wendell.html

Wheeler, B. (Ed.). (2015). Music therapy handbook. New York: Guilford Publications.

Wheeler, B. (2016). Music therapy research: An overview. In J. Edwards (Ed.), The oxford handbook of music therapy (pp. 720-747). Oxford, UK: Oxford 
University Press.

White, S., \& Seibold, C. (2008). Walk a mile in my shoes: An autoethnographic study. Contemporary Nurse, 30, 57-68.

Whitehead-Pleaux. (2017a). Discrimination and oppression. In A. Whitehead-Pleaux \& X. Tan (Eds.), Cultural intersections in music therapy: Music, health, and the person. Dallas: Barcelona Publishers.

Whitehead-Pleaux. (2017b). Discrimination and Oppression. In A. Whitehead-Pleaux \& X. Tan (Eds.), Cultural intersections in music therapy: Music, health, and the person. Dallas: Barcelona Publishers.

Whitehead-Pleaux, A., Donnenwerth, A., Robinson, B., Hardy, S., Oswanski, L., Forinash, M., ... York, E. (2012). Lesbian, gay, bisexual, transgender, and questioning: Best practices in music therapy. Music Therapy Perspectives, 30(2), $158-166$.

Williams, C. (1999). African American women, Afrocentrism and feminism. Women \& Therapy, 22(4), 1-16. http://doi.org/10.1300/J015v22n04

Williams, M. (2003). To be or not to be disabled. In M. Banks \& E. Kaschak (Eds.), Women with visible and invisible disabilities: Multiple intersections, multiple issues, multiples therapies. (pp. 145-154). London: The Haworth Press, Inc.

Wolbring, G. (2008). The politics of ableism. Development, 51, 252-258. http://doi.org/10.1057/dev.2008.17

Wolbring, G. (2009). What next for the human species? Human performance enhancement, ableism and pluralism. Development Dialogue, 2(august), 141163.

Wolbring, G. (2012). Expanding ableism: Taking down the ghettoization of impact of disability studies scholars. Societies, 2, 75-83. http://doi.org/10.3390/soc2030075

Woodward, A. (2015). Tapestry of tears: An autoethnography of leadership, personal transformation, and music therapy in humanitarian aid in Bosnia Herzegovina (unpublised doctoral dissertation). Antioch University. 
Woolf, V. (1930). On being ill. Britain: The Hogarth Press.

World Health Organisation. (2011). World report on disability. Malta. Retrieved from http://www.who.int/disabilities/world_report/2011/report.pdf

Yates, S., \& Hiles, D. (2010). Towards a “critical ontology of ourselves"?: Foucault, subjectivity and discourse analysis. Theory \& Psychology, 20(1), 52-75. http://doi.org/10.1177/0959354309345647

Young, I. (2000). Disability and the definition of work. In L. Francis \& A. Silvers (Eds.), Americans with disabilities: Exploring implications of the law for individuals and institutions. New York: Routledge.

Young, S. (2014). I'm not your inspiration, thank you very much. Sydney, Australia: TED: Ideas worth spreading. Retrieved from https://www.ted.com/talks/stella_young_i_m_not_your_inspiration_thank_you_ very_much

Zerubavel, N., \& O'Dougherty-Wright, M. (2012). The dilemma of the wounded healer. Psychotherapy, 49(4), 482-491.

Zhang, X., Shi, R., \& Hsu, W. (2016). A collaborative autoethnographic exploration of experiences of three international music therapy interns during their clinical training (unpublished masters thesis). State University of New York.

Ziebland, S., \& Coulter, A. (2013). Introduction. In S. Ziebland, A. Coulter, J. Calabrese, \& L. Locock (Eds.), Understanding and using health experiences: Improving patient care. (pp. 1-5). Great Britain: Oxford University Press.

Zola, I. (1993). Disability statistics: What we count and what it tells us: A personal and political analysis. Journal of Disability Policy Studies, 4(2), 9-39. 


\title{
Appendices
}

\section{Appendix 1: Examples of information and consent forms}

\author{
TE KÖKITNEW ZEALAND SCHOOL OF MUSIC \\ VICTORIA UNIVERSITY OF WELLINGTON, PO Box 600, Wellington 6140, New Zealand \\ Phone +64-4-463-5369 Email music@nzsm.ac.nz Web www.nzsm.ac.nz
}

\section{Supervisors information sheet}

Research Working Title:

An autoethnography of a music therapist practitioner-patient: Exploring the counterpoint.

Dear

I am writing to ask permission to analyse and write about an experience/correspondence we may have shared together, as part of my $\mathrm{PhD}$ research. My research process will involve reflecting on my experiences and writing about these.

For my research, I have chosen to explore how the close examination of a music therapist's personal experience with illness/disability can impact on practice, how the work can be negotiated, and to uncover any unique insight such a narrative may shed about therapeutic process. This research will help therapists, as it endeavourers to illuminate how societal ideas about illness and disability may impact on the therapeutic process, support ways of integrating professional and personal knowledge, and to open up new meanings of practice.

It is important to be aware that because I am identifiable as the researcher, as my supervisor you may not be granted anonymity (even with a pseudonym, you may be identifiable through your relationship with me). To ensure that you are comfortable with what is written I will share with you anything I write that involves you in some way. You will have the opportunity to say if you don't like what is written and can request that it is changed or omitted. It is ok to say no to me using an experience/correspondence we have shared for this research. Our supervision arrangements will remain the same. Your consent is voluntary. It is your right to withdraw information from the research up until the end of data analysis. The proposed date for this is the first of June 2018. 
All written records and consent forms for this research will be stored in a locked cupboard at the New Zealand School of Music. Electronic information will be kept on a password-protected computer. All records will be kept for five years and access will be restricted to the investigator and her supervisors. After five years it will be destroyed.

I will present the research findings to my examiners at the end of my $\mathrm{PhD}$ and I may also present the research at other conferences, if appropriate. The findings may be published in suitable music therapy or other prófessional journals. Victoria University library will have available copies of the thesis and it will also be available in an electronic repository and it will also be available in an electronic repository.

The Victoria University Human Ethics Committee have given approval for this research. Sarah Hoskyns and Sue Cornforth will supervise the project. If you have any concerns about the conduct of this research, please contact one of my supervisors: Sarah Hoskyns (ph, 044635233 x 35807 or email, sarah.hoskyns@nzsm.ac.nzor); Sue Cornforth, (ph, 043849516 or email Sue.Cornforth@vuw.ac.nz). If you wish to raise an issue with someone other than the researcher or supervisor, please contact the Victoria University Human Ethics Committee Convenor AProf Susan Corbett, email susan.corbett@vuw.ac.nz, phone+64-4-463 5480.

If you are in agreement with me examining and writing about an experience/s we shared, please sign both copies of the enclosed consent form and return one

to

Yours sincerely,

Carolyn Shaw

Registered Music Therapist

PhD Student

Victoria University

New Zealand School of Music

Ph: 0211323651 


\section{Supervisor consent form}

Research title: An autoethnography of a music therapist practitioner-patient: Exploring the counterpoint.

- I have read the information sheet and have obtained sufficient information about the study

- I understand that my real name will not be used in any publication or presentation arising from this research.

- I understand that Carolyn will share pieces of writing that involve me in any way and that I will have the opportunity to say if I don't like what is written and can request that it is changed or omitted. She will do this because I may be identifiable through our relationship.

- I understand that research data will be kept for five years and will be stored securely at the New Zealand School of Music and/or on a passwordprotected computer.

- I understand that I can withdraw information from research up until the $01 / 06 / 2018$.

- I acknowledge that the study may be presented at conferences and/or in published papers, and will be published in the library at Victoria University.

- I understand that I can contact Carolyn's research supervisors if I have any concerns or questions relating to the research.

- I also understand that I can contact the Victoria University of Wellington Human Ethics Convenor if I have any other concerns about this research

\section{YES/NO}

I therefore give consent for Carolyn to analyse and write about an experience we may have shared together for the purpose of her $\mathrm{PhD}$ research.

This has been discussed with me by

Signed date:

Print name 


\section{Facility information sheet}

\section{Research Working Title:}

An autoethnography of a music therapist practitioner-patient: Exploring the counterpoint.

Dear

I am writing to you to ask permission to review my clinical notes and records that I have kept during my work at for my

$\mathrm{PhD}$ research. It is possible that I may also seek your assistance in approaching families for informed consent should a particular example be needed to illuminate the findings. This would involve taking parents and students through the information sheets (please find attached) and asking permission from the parent and child.

For my $\mathrm{PhD}$ research, I have chosen to explore how the close examination of a music therapist's personal experience with illness/disability can impact on practice, how the work can be negotiated, and to uncover any unique insight such a narrative may shed about therapeutic process. This research will help therapists, as it endeavourers to illuminate how societal ideas about illness and disability may impact on the therapeutic process, support ways of integrating professional and personal knowledge, and open up new meanings of practice.

For ethical reasons, the data generated from clinical data mining will be represented in the research as "composite cases" that are emotionally rendered representations of experiences using fictional characters and settings. Thus ensuring the confidentiality of the students I have worked with. The importance is the essence of the findings as opposed to the precise detail. If for some reason a particular case is needed to illuminate the findings, and a composite case is not used, families will be approached for consent. 
All written records and consent forms for this research will be stored in a locked cupboard at the New Zealand School of Music. Electronic information will be kept on a password-protected computer. All records will be kept for five years and access will be restricted to the investigator and her supervisors. After five years it will be destroyed. It is your right to withdraw information from the research up until the end of data analysis. The proposed date for this is the first of June 2018.

I will present the research findings to my examiners at the end of my $\mathrm{PhD}$ and $\mathrm{I}$ may also present the research at other conferences, if appropriate. The findings may be published in suitable music therapy or other professional journals. Victoria University library will have available copies of the thesis and it will also be available in an electronic repository.

The Victoria University Human Ethics Committee have given approval for this research. Sarah Hoskyns and Sue Cornforth will supervise the project. If you have any concerns about the conduct of this research, please contact the supervisor or, if you wish to raise an issue with someone other than the researcher or supervisor, please contact the Victoria University Human Ethics Committee Convenor AProf Susan Corbett, email susan.corbett@vuw.ac.nz, phone+64-4-4635480.

If you are in agreement with me reviewing records generated at your facility/school, please sign both copies of the enclosed consent form and return one to me. Please do not hesitate to contact my primary research supervisor, Sarah Hoskyns, if you have any questions or wish to discuss this further. Ph: 044635233 x 35807 or email: sarah.hoskyns@nzsm.ac.nz

Yours sincerely,

Carolyn Shaw

NZ Registered Music Therapist

$\mathrm{PhD}$ Student Victoria University

Ph: 0211323651 or email: *** 


\section{Facility consent form}

\section{Research title: An autoethnography of a music therapist practitioner-patient:} Exploring the counterpoint.

- I have read the information sheet and have obtained sufficient information about the study.

- I understand that from the review of clinical notes students' real names and details will not be used in any publication or presentation arising from this research.

- I understand that informed consent will be sought from families should a particular case vignette be needed to illustrate the findings.

- I understand that research data will be kept for five years and will be stored securely at the New Zealand School of Music and/or on a passwordprotected computer.

- I understand that I can withdraw information from research up until the 01/06/2018.

- I acknowledge that the study may be presented at conferences and/or in published papers, and will be published in the library at Victoria University.

- I understand that I can contact Carolyn's research supervisor Dr Sarah Hoskyns if I have any concerns or questions relating to the research.

- I also understand that I can contact the Victoria University of Wellington Human Ethics Convenor if I have any other concerns about this research.

\section{YES/NO}

I therefore consent to Carolyn Shaw reviewing clinical notes and records that were kept during her work at for her research.

This has been discussed with me by Carolyn Shaw (researcher).

Signed Date:

Print name 


\section{Parent/caregiver information sheet}

\section{Research Working Title:}

An autoethnography of a music therapist practitioner-patient: Exploring the counterpoint.

Dear (parent/caregiver),

I am writing to you to ask permission to review. 's (your child's) music therapy notes, videos, and music as part of my $\mathrm{PhD}$ research and to write and present what I have learnt. I will be looking at the recordings and notes to learn about my own approach, rather than studying (name).

For my $\mathrm{PhD}$ research, I have chosen to explore how illness/disability can affect how a therapist thinks about and does her work. This research will help all therapists, as it will discuss how society views disability/illness and how this affects what therapists do. This can open up new ways of doing things. It will help us understand how professionals can use personal knowledge in helpful ways.

I would like you to know that if your child is still receiving music therapy there would be no changes to .'s music therapy. This research will be discussed with your child in a way they can understand and I will not proceed without their consent/assent. Please be assured your family's consent for this is voluntary. There will be other people I can ask if you want to say no.

We will keep all the notes and consent sheets in a locked cupboard and computer records will have a protected password so that only I can access them. The notes and consent forms will be kept securely for five years at the New Zealand School of Music. I will give a different name for and will not mention your family or the school's name or location details in any publication or presentation of the research. If you change your mind about giving consent, you can do so up to the end of my detailed study ( $1^{\text {st }}$ of January 2018). 
I will present my research in a book for my examiners at the end of my $\mathrm{PhD}$ and I may also present the research at other conferences. The findings may be published in a professional journal. Victoria University library will have available copies of the book and it will also be available in an electronic repository. You can have a copy of the results of the research and the section that applies to if you request it.

The Victoria University Human Ethics Committee has given approval for this research. Sarah Hoskyns and Sue Cornforth will supervise the project. If you have any concerns about the conduct of this research, please contact my primary supervisor or, if you wish to raise an issue with someone other than the researcher or supervisor, please contact the Victoria University Human Ethics Committee Convenor AProf Susan Corbett, email susan.corbett@vuw.ac.nz, phone +64-4-463 5480.

If you are happy to say yes to me using ..'s music therapy records, please sign both copies of the enclosed consent form and return one to

Please contact me or my primary research supervisor,

Sarah Hoskyns, if you have any questions or wish to discuss this further. Ph: 044635233 x 35807 or email: sarah.hoskyns@nzsm.ac.nz

Yours sincerely,

Carolyn Shaw

NZ Registered Music Therapist

$\mathrm{PhD}$ Student

Victoria University

$\mathrm{Ph}: 0211323651$ or email: *** 


\section{Parent/caregiver consent form}

\section{Research title: An autoethnography of a music therapist practitioner-patient:} Exploring the counterpoint.

- I have read the information sheet and have obtained sufficient information about the study

- I understand that my child's real name will not be used in any publication or presentation arising from this research.

- I understand that research data will be kept for five years and will be stored securely at the New Zealand School of Music and/or on a password protected computer.

- I understand that I can withdraw information from research up until the 01/06/2018.

- I acknowledge that the study may be presented at conferences and/or in published papers, and will be published in the library at Victoria University.

- I understand that Carolyn will de-identify material relating to my child and family in reports and therefore we will not be identifiable.

- I understand that I can contact Carolyn's research supervisor Dr Sarah Hoskyns if I have any concerns or questions relating to the research.

- I also understand that I can contact the Victoria University of Wellington Human Ethics Convenor if I have any other concerns about this research.

\section{YES/NO}

Therefore I give consent for the following materials related to my child's music therapy sessions to be used in a case vignette to illustrate the findings of the research:

I agree/do not agree (please circle one) to the use of my child's clinical notes and written material.

I agree/do not agree (please circle one) to the use of my child's session videos.

I agree/do not agree (please circle one) to audio recordings of my child's songs/improvisations.

This has been discussed with me by (teacher)

Signed Date:

(caregiver/s)

Print name 
TE KŌKI NEW ZEALAND SCHOOL OF MUSIC

VICTORIA UNIVERSITY OF WELLINGTON, PO Box 600, Wellington 6140, New Zealand

\section{$\underline{\text { Student information sheet }}$}

\section{Research Working Title:}

An autoethnography of a music therapist practitioner-patient: Exploring the counterpoint.

Dear (child),

I am going to write a story about doing music with children. I am writing to ask if you would like to be part of this story. I am asking you because we have played music together before, or still play music together now. I am writing this story to help me understand our time together. The story will talk about our instrument playing, singing, dancing, and the songs we made up together.

I will share this story with mums, dads, teachers, and other people, so they too can understand what we do together. It will not have your real name in it. It is OK if you don't want to be in the story, there are other children I can ask. If you are unsure or don't understand, you can ask lots of questions about the story project before deciding.

If you do want to be in my story you need to say "OK" to the teacher, and if you can, write your name on the paper behind this one. Someone will write your name if it is too hard.

If you say "OK" I will be happy. If you don't say "OK”, I will still be happy. Thank you for reading or listening to my letter.

All the best,

Carolyn Shaw

Music lady 


\section{Child consent/assent form}

\section{Research title: An autoethnography of a music therapist practitioner-patient:} Exploring the counterpoint.

This consent will be held for five years

Someone has read me the letter about Carolyn's story and I understand what it is about. I have had time to think about whether I want to be in the story and I have asked all my questions.

It is OK for Carolyn to write a story about the music we did together and to show this to other people.

Signature date:

Full name printed: 


\section{Appendix 2: Co-written music therapy report example}

\section{Music Therapv Report}

Date: December 2015

Student: Callum

Carolyn and Callum have written this report together. Callum's writing appears in the text boxes throughout.

This is a pilot report to see what I thought of music. I will write a proper one next year when I've had more time.

Callum, you started music therapy sessions in term two. Initially you came individually so we could discuss the potential direction of sessions. You then decided that you would like other students from your class to join you. Group sessions then commenced.

Areas and possibilities explored in sessions 2015

\section{1) Creating an environment of shared fun and enjoyment}

We had a good time exploring ways that play can be satisfying and fun.

I like to bring people to music because it's more fun. It makes the games harder, but more fun.

Callum, you liked to invent games with a lot of rules that the other students and I had difficulty following at times. We tested out how it felt to play music with many rules, some rules, and music making that was free from all rules. Each group member had a different preference for what they found more enjoyable. Due to this, the group felt it was fair if they played a range of games/music and to not get "stuck" in just one way of playing.

If someone was losing interest in the middle of a game, it was helpful to stop the game to discuss what was happening. Callum, you came up with solutions of how you could support others to stay engaged once you were aware of what was happening. For example, while playing 'sneak up grandma', you stopped making people go back to the beginning for breathing. This enabled everyone to enjoy progressing in the game. I encouraged you to say when you were finding something difficult instead of getting upset. Such as, when you found another's music making intrusive, you asked the other player if she could lower her volume. Discussions occurring in the moment of the incident seemed to support social understanding.

I'm happy if we can make it so we can pick some games before each week. Then we don't have to spend a lot of time thinking of a game. So we can know exactly what we are going to play at a certain time. It feels uncomfortable and weird if we don't know what we are going to do.

We changed the structure of the sessions so that the group planned what they would like to do at the beginning. You seemed to be more comfortable when the uncertainty 
of what we might do was reduced and the maximum time was utilised. Callum, you were flexible with spontaneous changes to the plan.

It was OK to play Liam's new game (:) of guess the instrument. It was nice to learn a new game today.

I don't mind waiting when others are playing. I wanted to play for different amounts of time each time when it was my turn.

Everyone developed many creative musical games together and this sparked a lot of fun and collaboration. One player from your class wanted to put in this report that: "Everyone has different ideas for making up good games. It was cool to see how they would turn out. I think we made something really cool". This indicates that they shared a positive joint experience.

\section{2) Developing understanding and friendship with peers}

Callum you liked to rotate the students you chose to come with you. However, there seemed to be a core group in particular that you invited frequently.

I like to get to know other people. Like last week Greta said she had been rock climbing. Today was fun because I brought in some people I usually don't bring Brooke and Luke. I get to spend some time with people I really like and know.

Some activities focused on players developing understanding of others. You regularly asked to play one activity in particular. In this activity, one player played an emotion on their instrument and the group had to figure out what it was. We then had to think of what might make that person feel that way. This sparked interesting discussions. One player mentioned that you get mad when someone takes your special cushion off your seat. They were aware of your triggers, Callum, and were able to explain why it might make you upset. You were able to think of situations that might make other people feel a certain way, however, these tended to be less specific and based on your personal experience rather than what you knew of the other person. However, Callum, you were really engaged in this game and keen to hear about others' experiences.

\section{Thoughts for 2016}

I do wish we could have more room somehow. It would be nice to have different songs every time we come to music for hello and goodbye. Different songs for different feelings, so different hello and goodbye songs depending on how we are feeling.

I want to do music next year. It was really fun. I'd like to play better than I already can. I would like to play the games better and it also brings friendship up from other people and it means others want to come and play with me, which is actually really rare.

You indicated that you value the relational opportunities sessions provide. Therefore, we will continue to explore the possibilities for enhancing the above areas of focus in 
2016. Your idea to include more potential for emotional expression within the hello and goodbye activities will be implemented.

In speaking with Jill and Jackie, I am happy to support, where possible, your natural music talent Callum. You have shown great skill in the recorder and from our

discussions you are interested in learning a wind instrument or another instrument like

the violin or drums. However, you mentioned that you would like to know more about other options.

Carolyn and Callum

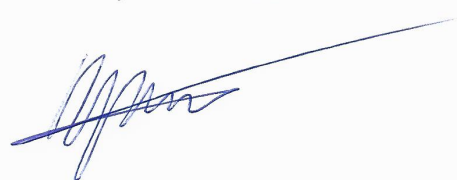




\title{
Appendix 3: Notes and recommendations to the Standards of Practice Committee
}

\author{
Standards of Practice Working Party \\ Notes from meeting with Claire Molyneux and Carolyn Ayson \\ Re: MThNZ Standards of Practice and Code of Ethics \\ Friday 24 June 201610.30 - 11.30am
}

Purpose of meeting:

To have initial discussion about the Standards of Practice and Code of Ethics documents with a view to offering guidance around areas that need review and to support the working party with understanding contemporary views of disability and the use of language in the documents.

Summary of main points of discussion

The discussion focused on the following questions:

1. How do the documents include/exclude understandings of disability and illness?

2. How do the documents position people with disability and illness?

3. What is the philosophical position (of MThNZ) that underpins the documents and is the wording congruent with this position?

Some detailed discussion took place about the two documents and the main points of this discussion are summarised below. However, before reviewing the finer detail of the documents, it is our recommendation that MThNZ considers adding a statement that makes it clear how disability, illness, health and well-being is viewed in the documents. This statement will indicate the ways in which music therapists take into account the relational and environmental aspects of their work with clients. We have proposed some options below for the working party to consider (ACTION POINT 1)

Statement 1

It is expected that music therapists in New Zealand will have an understanding of a range of different models of health, well-being, disability and illness. For the purposes of these documents, MThNZ finds interactive approaches that acknowledge the interaction of biological, social, cultural, psychological, and environmental factors that dynamically produce health, well-being, disability, and illness as helpful in informing the practice of music therapy as a process that can create change for individuals and communities.

Or:

\section{Statement 2}

As there is no unitary perspective of disability, health, wellbeing, and illness, it is expected that music therapists in New Zealand will have an understanding of a range of models and be able to support a plurality of perspectives. For the purposes of these documents, MThNZ finds interactive approaches that acknowledge the interaction of biological, social, cultural, psychological, and environmental factors that dynamically produce health, well-being, disability, and illness as helpful in informing the practice of music therapy as a process that can create change for individuals and communities.

Discussion also took place about Carolyn's role and she expressed a wish to be present at meetings in order to contribute to discussions around these issues. We would therefore like to recommend that Carolyn is invited to join the working party (ACTION POINT 2). 


\begin{tabular}{|c|c|}
\hline \multicolumn{2}{|c|}{ Standards of Practice } \\
\hline Discussion point & $\begin{array}{l}\text { Recommendations } \\
\text { (ACTION POINT 3) }\end{array}$ \\
\hline $\begin{array}{l}\text { 1. Carolyn observed there are } \\
\text { incongruencies between the } \\
\text { Standards of Practice (SoP) } \\
\text { document and the } 2000 \text { NZ Disability } \\
\text { Strategy (NZDS) due to three main } \\
\text { reasons: } \\
\text { Disability is framed more from a } \\
\text { social model perspective in NZDS } \\
\text { whereas in the SoP it is } \\
\text { predominantly medically framed. } \\
\text { Partnership is often referred to as } \\
\text { important in NZDS whereas in SoP } \\
\text { this focus is lacking within the } \\
\text { therapeutic process for People With } \\
\text { Disabilities (PWD). } \\
\text { Expert position is placed heavily on } \\
\text { therapist in SoP, whereas NZDS } \\
\text { emphasise the need to "recognise that } \\
\text { disabled people are experts on their } \\
\text { own experience". }\end{array}$ & $\begin{array}{l}\text { MThNZ to decide how to } \\
\text { frame disability and illness } \\
\text { with the recommendation } \\
\text { that a move towards a more } \\
\text { inclusive model that } \\
\text { acknowledges the } \\
\text { interaction of different } \\
\text { elements (e.g. bio-psycho- } \\
\text { social) that influence } \\
\text { perception and experience of } \\
\text { disability and illness (as } \\
\text { discussed above and in point } \\
5 \text { below). } \\
\text { Partnership to have more } \\
\text { focus (see point } 3 \text { below for } \\
\text { further elaboration). } \\
\text { Include the expertise of } \\
\text { PWD in therapy processes } \\
\text { (see point } 4 \text { below for } \\
\text { further detail) }\end{array}$ \\
\hline $\begin{array}{l}\text { 2. Due to the predominant medical } \\
\text { framing, the cultural competence section } \\
\text { does not specifically mention disability } \\
\text { culture. It needs to acknowledge other } \\
\text { cultures within which music therapists } \\
\text { typically work such as different disability } \\
\text { cultures. Discussion took place about the } \\
\text { challenge of whether to specify particular } \\
\text { cultures or not and how naming some } \\
\text { cultures can act to exclude others. }\end{array}$ & $\begin{array}{l}\text { Review cultural competence section } \\
\text { with an emphasis on inclusive } \\
\text { statements such as "respect for } \\
\text { diverse cultural backgrounds of the } \\
\text { populations with which the music } \\
\text { therapist works". }\end{array}$ \\
\hline $\begin{array}{l}\text { 3. Partnership is referenced in the section on } \\
\text { cultural competence, specifically in relation } \\
\text { to the Treaty of Waitangi. Partnership with } \\
\text { clients is important and should be } \\
\text { highlighted e.g. recognising that disabled } \\
\text { people are the experts of their own } \\
\text { experience, planning collaboratively with } \\
\text { clients and enhancing participation. }\end{array}$ & $\begin{array}{l}\text { Review the emphasis on partnership } \\
\text { and participation in relation to all } \\
\text { clients. Review the definition of } \\
\text { cultural competence to be inclusive } \\
\text { of other cultures such as different } \\
\text { disability cultures. }\end{array}$ \\
\hline $\begin{array}{l}\text { 4. The sections 'Assessment' 'Intervention' } \\
\text { and 'Evaluation' position the music therapist } \\
\text { as expert with no inclusion of the client's } \\
\text { expertise. In these sections statements } \\
\text { suggest that the therapist is in direct control }\end{array}$ & $\begin{array}{l}\text { Review these sections with } \\
\text { language that produce other } \\
\text { relations between therapists and } \\
\text { whom they work. }\end{array}$ \\
\hline
\end{tabular}


over their clients: they do something to someone else.

Note that the word 'intervention' is

becoming outdated within music therapy

texts and has been omitted from Bruscia's

latest definition (Bruscia, 2014 "Defining

music therapy"). It was omitted due to its

unintended but undesirable connotations of

power, hierarchy, and client passivity. These are important to consider in documents that impact on PWD. Additionally, 'intervention' does not work for all models of music therapy..

5. Point 3. b) does not include a requirement to demonstrate awareness and understanding of different perspectives of disability and illness. It instead implies that there is one "right" way therapists can

distinguish/identify between health, illness, disability etc - that it is an object that can be captured.

6. Do we want to be encouraging reflexive rather than reflective practitioners (or both)? Reflexivity addresses imbalance of power mentioned above for PWD, as a reflexive process changes the therapist. Reflexivity also supports social, cultural aspects of disability/illness and practice as it requires the therapist to look outward to cultural, historical, political forces that shape their practice.
Consider replacing

"intervention", with something like

"approaches" for example.

Review

Review sections of both documents that refer to reflective practice and consider defining terms such as reflective and reflexive.

\begin{tabular}{|c|c|}
\hline \multicolumn{2}{|c|}{ Code of Ethics } \\
\hline Discussion point & $\begin{array}{l}\text { Recommendation } \\
\text { ACTION POINT } 4\end{array}$ \\
\hline $\begin{array}{l}\text { 1. Carolyn observed that this document } \\
\text { places harm clearly between } \\
\text { individuals (therapist and client) and } \\
\text { does not take account of unjust } \\
\text { social contexts (harm from } \\
\text { circumstances external to the client } \\
\text { and therapist). Thus allowing little } \\
\text { scope for advocacy, community }\end{array}$ & $\begin{array}{l}\text { MThNZ to decide how to frame } \\
\text { disability and illness with the } \\
\text { recommendation that a move } \\
\text { towards a more inclusive model that } \\
\text { acknowledges the interaction of } \\
\text { different elements (e.g. bio-psycho- } \\
\text { social) that influence perception and } \\
\text { experience of disability and illness. }\end{array}$ \\
\hline
\end{tabular}




\begin{tabular}{|l|l|}
\hline \multicolumn{1}{|c|}{$\begin{array}{c}\text { well-being and valuing of social } \\
\text { justice in ethical thinking. }\end{array}$} & \\
\hline $\begin{array}{l}\text { 2. Overall, the headings in the document } \\
\text { don't seem to clearly fit the statements } \\
\text { and need careful review to distinguish } \\
\text { what is meant by 'relationships with" } \\
\text { and 'responsibilities to". }\end{array}$ & Review headings \\
\hline $\begin{array}{l}\text { 3. The statement at } 4 \text { b) "maintain adequate } \\
\text { standards of physical, emotional, and } \\
\text { mental health and seek help, or refrain } \\
\text { from working with clients if his or her } \\
\text { fitness is impaired" is exclusionary. It } \\
\text { only marks illness and illness related } \\
\text { disability as a potentially dangerous } \\
\text { state in which to practice. It does not } \\
\text { engender its inclusivity as one of the } \\
\text { many human experiences that can have } \\
\text { implications (positive and negative) for } \\
\text { practice. }\end{array}$ & \\
\hline
\end{tabular}

\section{Action points}

1. Working Party and MThNZ to review and make explicit how disability, illness, health and well-being is viewed in the Standards of Practice and Code of Ethics documents.

2. Working Party to consider recommendation that Carolyn is invited to join the group.

3. Working Party and MThNZ to consider the recommendations outlined in this document, plan timeline and necessary consultation to review Standards of Practice.

4. Working Party and MThNZ to consider the recommendations outlined in this document, plan timeline and necessary consultation to review Code of Ethics. 